\title{
Coal Production 1989
}

\section{Energy Information Administration}

Office of Coal, Nuclear, Electric and Alternate Fueis

U.S. Department of Energy

Washington, DC 20585

\section{MASTER}

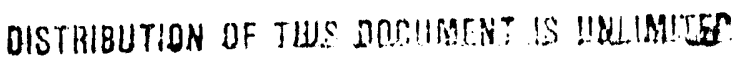

This report was prepared by the Energy Information Administration, the independent intatistical and arlaiyuicai agency within the Depariment of Energy. The inionnation contained herein should not be construed as advocating of reflecting any policy position of the Department of Energy or any other organization. 


\section{Contacts}

General information for this document may be obtained from Robert M. Schnapp (202/254-5392), Di. rector of the Coal Division, Office of Coal, Nuclear, Electric and Alternate Fuels, Energy Information Administration, or from Noel C. Balthasar (202/254-5400), Chief of the Coal Data Systems Branch.
Specific information about the data in this report may be obtained from Bruce F. Quade (202/254-5461), John G. Colligan (202/254-5465), Clyde E. Boykins (202/254-5406), Jerome A. Saaderson (202/254-5463), and Joyce E. Morrison (202/254-5468). For information about the feature article, contact Eugene R. Slatick (202/254-5384) and Michelle Bowles (202/254-5378). For specific information about the demonstrated reserve base data, contact Richard Bonskowski $(202 / 254-5383)$. 


\section{Preface}

Coal Production 1989 provides comprehensive information about U.S. coal production, the number of mines, prices, productivity, employment, reserves, and stocks to a wide audience including Congress, Federal and State agencies, the coal industry, and the general public. The data presented in this report were collected and published by the Energy Information Administration (EIA), to fulfill its data collection and dissemination responsibilities as specified in the Federal Energy Administration Act of 1974 (Public Law 93-275), as amended.

The 1989 coal production and related data presented in this report were obtained from Form EIA-7A, "Coal Production Report," submitted by companies owning mining operations that produced, processed, or prepared 10,000 or more short tons of coal in 1989. This survey originated at the Bureau of Mines, U.S. Department of the Interior. In 1977, the responsibility for taking the survey was transferred to the EIA under the Department of Energy Organization Act (Public Law 95-91). The data cover 3,511 of the 4,357 U.S. coal mining operations active in 1989. These mining operations accounted for over 99 percent of total U.S. coal production and represented 81 percent of all U.S. coal mining operations in 1989. This issue is the 14th annual report published by EIA and continues the series formerly included as a chapter in the Minerals Yearbook published by the Bureau of Mines.

This report also includes data for the demonstrated reserve base of coal in the United States on January 1, 1990. This is the 10th annual summary on minable coal, pursuant to Section 801 of Public Law 95-620, the Powerplant and Industrial Fuel Use Act of 1978.

The Office of Coal, Nuclear, Electric and Alternate Fuels gratefully acknowledges the cooperation of the respondents in supplying the information presented in this report and appreciates the valuable assistance of the State coal mining agencies, the State geological and mineral survey offices, the U.S. Geological Survey, U.S. Department of the Interior, and the Mine Safety and Health Administration, U.S. Department of Labor.

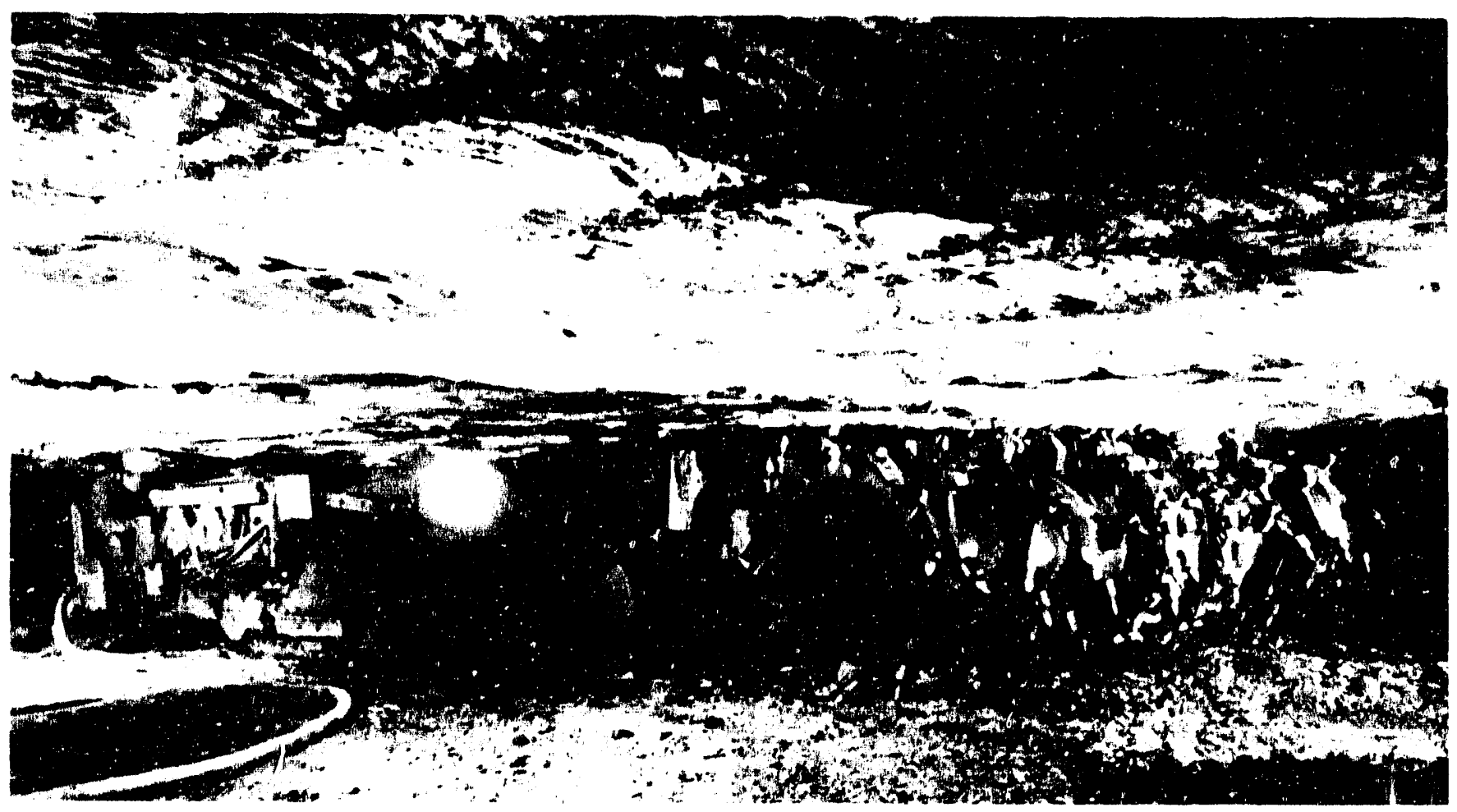

A continuous mining machine, capable of mining 81015 tons of coal per minute, is being moved to a new coal face. 


\section{Contents}

Feature Article: U.S. Coal Production in the 1980's: An Overview . . . . . . . . . . . . . .

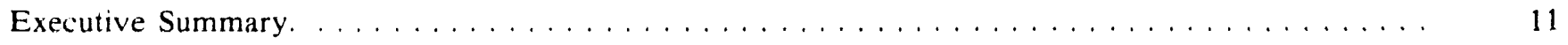

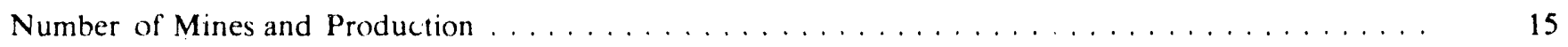

Average Mine Prices . . . . . . . . . . . . . . . . . . . . . . . . . . . . . . .

Productivity and Employment $\ldots \ldots \ldots \ldots \ldots$

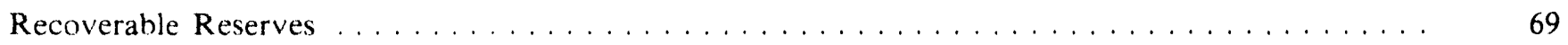

Appendices

A. Demonstrated Reserve Base . . . . . . . . . . . . . . . . . . . . . . . . 79

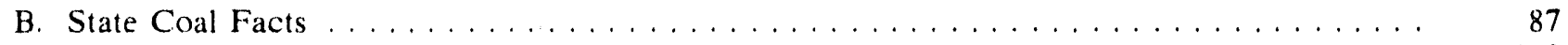

C. Reference Tables and Maps . . . . . . . . . . . . . . . . . . . . . . . . . . 123

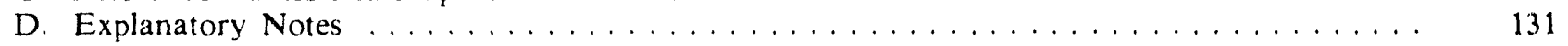

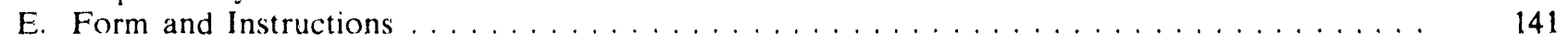

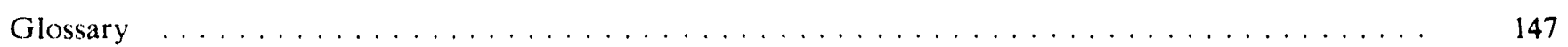




\section{Tables}

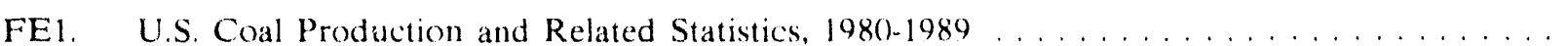

Page

FE2. Peak Year of Coal Production by State, from Earliest Records Through 1989 . . . . . . .

FE3. U.S. Coal Production, Disposition, and Prices, Selected Years, 1980-2000 . . . . . . . . .

1. Total U.S. Coal Production and Number of Mines by State and Type of Mining, 1989 . . . .

2. U.S. Coal Production and Number of Mines by State and Type of Mining, 1989 . . . . . . .

3. U.S. Coal Production and Number of Mines by District and Type of Mining, 1989 . . . . .

4. U.S. Coal Production and Number of Mines by State and County and Type of Mining, 1989

5. U.S. Coal Production and Number of Mines by State, Type of Mining, and Mine Production

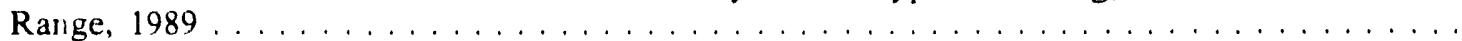

ó. U.S. Coal Production by State and Coal Rank, $1989 \ldots \ldots \ldots \ldots \ldots \ldots$

7. U.S. Production of Bituminous Coal by State and Coal Group, 1989 . . . . . . . . . . . .

U.S. Production of Subbituminous Coal by State and Coal Group, $1989 \ldots \ldots \ldots$

U.S. Production of Lignite Coal by State and Coal Group, $1989 \ldots \ldots \ldots$

Pennsylvania Anthracite Production by County and Coal Group, $1989 \ldots \ldots \ldots$. . . . . . . . .

U.S. Coal Production by Coalbed Thicknes: and Type of Mining, 1989 . . . . . . . . . . . . .

U.S. Coal Production and Coalbed Thickness by Major Coalbeds and Type of Mining, 1989

U.S. Coal Production by Mine Production Range and Type of Mining, 1988, 1989 . . . . . .

Number of U.S. Coal Mines by Mine Production Range and Type of Mining, 1988, 1989 . .

U.S. Coal Production by State and Type of Mining, 1988, 1989 . . . . . . . . . . . . . .

Number of U.S. Coal Mines by State and Type of Mining, 1988, $1989 \ldots \ldots$. . . . . . . . . .

U.S. Coal Production by District and Type of Mining, 1988, 1989 . . . . . . . . . . . . .

Number of U.S. Coal Mines by District and Type of Mining, 1988, $1989 \ldots \ldots \ldots$

U.S. Coal Production and Average Mine Price by State and Type of Mining, 1989 . . . . .

U.S. Coal Production and Average Mine Price by District and Type of Mining, 1989 . . . .

U.S. Coal Production and Average Mine Price by State and Disposition, 1989 .........

U.S. Coal Production and Average Mine Price by District and Disposition, $1989 \ldots \ldots \ldots$

U.S. Coal Production, Number of Mines and Average Mine Price by State and County, 1989

U.S. Coal Production and Average Mine Price by State and Coal Rank, 1989 . . . . . . .

Average Mine Price of U.S. Coal by State and Type of Mining, 1988, 1989 . . . . . . . . .

Average Mine Price (if U.S. Coal by District and Type of Mining, 1988, 1989 . . . . . . . .

Average Mine Price of U.S. Coal by Mine Production Range and Type of Mining, 1988, 1989

U.S. Coal Mining Productivity by State, $1989 \ldots \ldots \ldots \ldots \ldots \ldots$

U.S. Underground Coal Mining Productivity by State, $1989 \ldots \ldots \ldots \ldots \ldots \ldots$

U.S. Surface Coal Mining Productivity by State, $1989 \ldots \ldots \ldots \ldots \ldots \ldots$

U.S. Coal Mining Productivity by District, $1989 \ldots \ldots \ldots \ldots \ldots \ldots \ldots \ldots$

U.S. Underground Coal Mining Productivity by District, $1989 \ldots \ldots \ldots \ldots \ldots \ldots$

U.S. Surface Coal Mining Productivity by District, 1989

Average Number of Miners Working Daily at U.S. Coal Mines by State and Type of Mining, 1988, 1989

Average Number of Miners Working Daily at U.S. Coal Mines by District and Type of Mining, 1988, 1989

36.

Average Number of Miners Working Daily at U.S. Coal Mines by Mine Production Range and Type of Mining, 1988, 1989

U.S. Coal Mining Productivity by State and Type of Mining, 1988, $1989 \ldots \ldots \ldots$

38. U.S. Coal Mining Productivity by District and Type of Mining, 1988, $1989 \ldots \ldots \ldots$

39. Recoverable Coal Reserves and Average Recovery Percentage at U.S. Mines by State and Type of Mining, 1989

40. Recoverable Coal Reserves and Average Recovery Percentage at U.S. Mines by District and Type of Mining, 1989

41. Recoverable Coal Reserves at U.S. Mines by State and Type of Mining, 1988, 1989 . . . .

42. Recoverable Coal Reserves at U.S Mines by District and Type of Mining, 1988, 1989 . . .

43. Recoverable Coal Reserves at U.S. Mines by Mine Production Range and Type of Mining, 1988,1989 


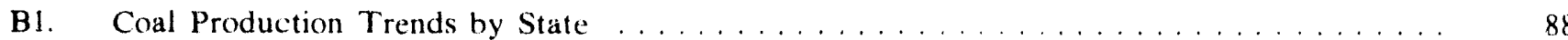

B2. Rankings for Coal Consumption, Reserves, and Production by Coal-Producing State . . . . 89

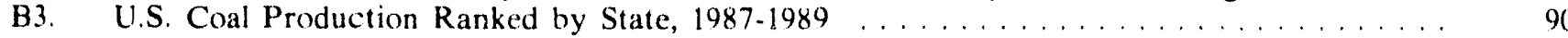

B4. U.S. Coal Consumption Ranked by State, 1987-1989 . . . . . . . . . . . . . . . . . . . 91

B5. U.S. Demonstrated Reserve Base on January 1, 1990, by Mining Method and Ranked by

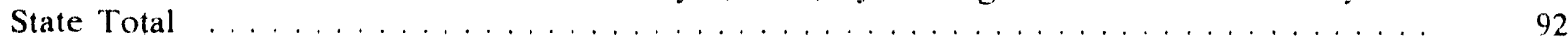

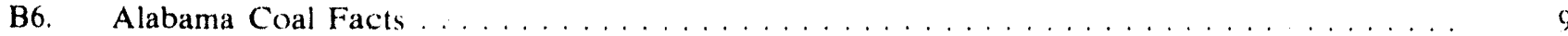

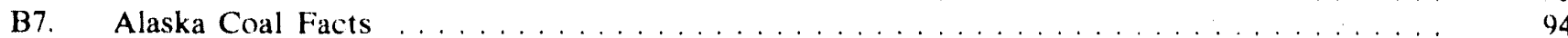

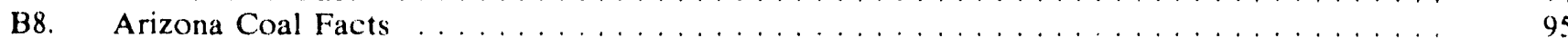

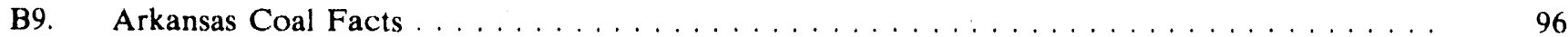

B10. California Coal Facts $\ldots \ldots \ldots \ldots \ldots$

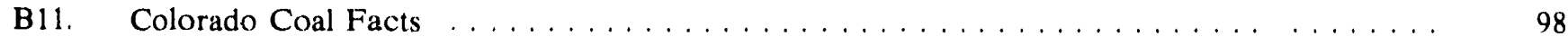

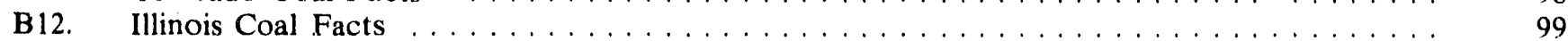

B13. Indiana Coal Facts $\ldots \ldots \ldots \ldots \ldots$

B14. Iowa Coal Facts $\ldots \ldots \ldots \ldots \ldots$

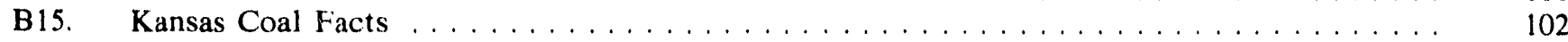

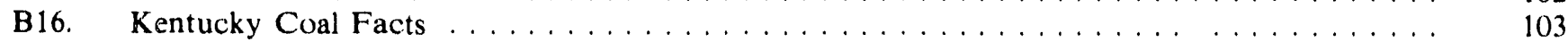

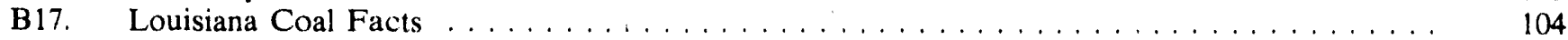

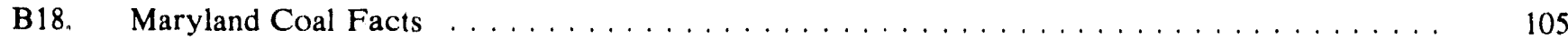

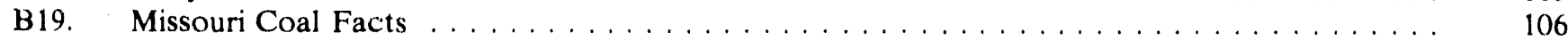

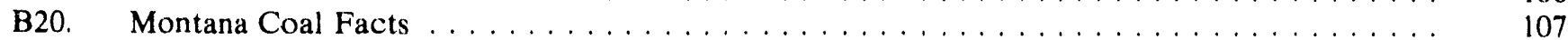

B21. New Mexico Coal Facts $\ldots \ldots \ldots \ldots \ldots$

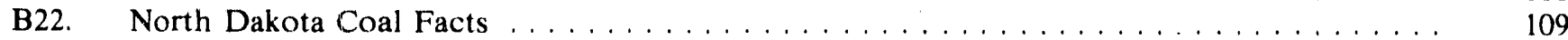

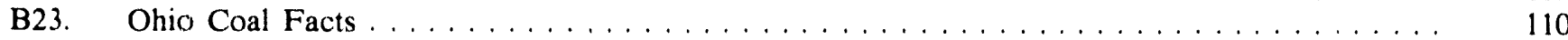

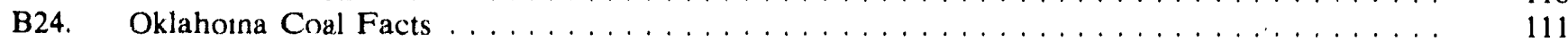

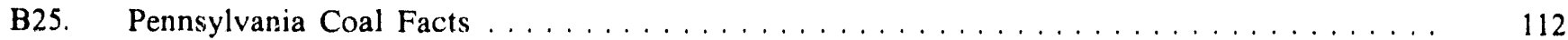

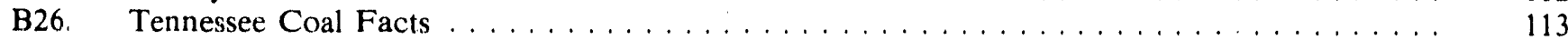

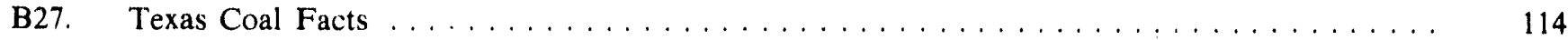

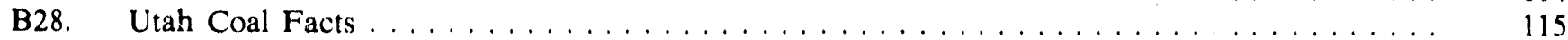

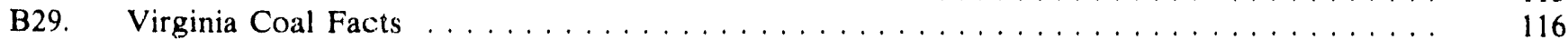

B30. Washington Coal Facts $\ldots \ldots \ldots \ldots \ldots \ldots$

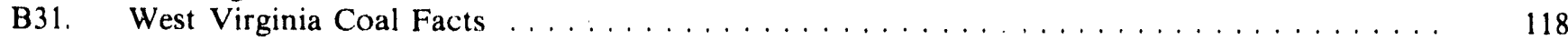

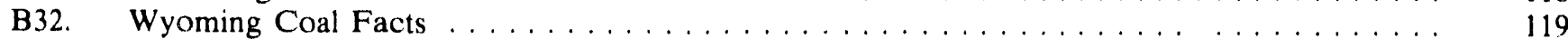

C1. Classification of Coals by $\mathrm{Rank} \ldots \ldots \ldots \ldots \ldots \ldots$

D1. Sources of Data for Total U.S. Coal Production and Number of Minirg Operations ...... 133

D2. Summary of EIA Revisions to Demonstrated Reserve Base of Coal . . . . . . . . . . . . . . 135

\section{Illustrations}

FE1. Trends in Coal Production and Demand in the $1980^{\prime} \mathrm{s} \ldots \ldots \ldots$

1. Coal Production by Region, $1989 \ldots \ldots \ldots \ldots \ldots \ldots$

2. U.S. Coal Production and Number of Mines by Type of Mining and by Region, 1980-1989 . 16

3. U.S. Coal Production by State, $1989 \ldots \ldots \ldots \ldots \ldots$

4. U.S. Coal Production by Coal Rank and Coalbed Thickness, 1980-1989 . . . . . . . . . .

5. Average Mine Price of U.S. Coal by Type of Mining and Coal Rank, 1980-1989 . . . . . . . 40

6. U.S. Coal Mining Productivity by Type of Mining and by Region, 1980-1989 . . . . . . . . . 56

7. Recoverable Coal Reserves at L'S. Mines by. Type of Mining and by Region, 1980-1989 . . 70

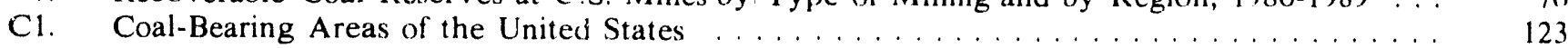

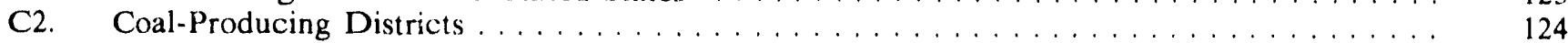




\title{
U.S. Coal Production in the 1980's: An Overview
}

\author{
by Eugene R. Slatick and Michelle D. Bowles
}

\section{Introduction}

The U.S. coal industry can lay claim to some impressive production statistics in the decade of the 1980's. The decade began with production rising above 800 inillion short tons for the first time in history. Annual production increased to new record levels five more times, reaching nearly 1 billion short tons at the end of the decade. Coal miners set new records for productivity every year, achieving this by working fewer mines with a work force that declined dramatically over the period. The price of coal declined during the decade, but it was generally higher than in the past, giving production an average annual value of over $\$ 21$ billion, more than twice as high as in the 1970's.

Nearly 9 billion short tons of coal--over 1 cubic mile of solid coal--were produced during the 1980's. This was one-third more than in the previous decade, and it raised the cumulative coal production for the last 100 years to about 54 billion short tons. Nevertheless, the remaining coal reserves are estimated to be abundant enough tc keep the Nation self-sufficient in coal well into the next century, and beyond, at the current rate of production.

\section{The Demand for Coal}

There was one overriding reason for the dramatic rise in U.S. coal production in the $1980^{\prime}$ s: a strong growth in the domestic demand for electricity. More than 7 out of every 10 tons of coal produced during the period were used to generate electricity. This was about 1 ton more than in the 1970's and 2 tons more than in the 1960's. Over half of the electricity generated annually in the 1980's was derived from coal.

The relationship between the coal industry and the electric utility industry dates back about a century, when the first practical electrical generating station went into operation. It has grown closer as technological strides raised the power generation levels from pulverized coal-firing, the principal coal-combustion method for generating electricity. Over the years, electric utilities have corsumed increasingly larger amounts of coal, and since the 1950's they have been the most important market for coal. Their reliance on coal has risen markedly since the 1970 's due to rising costs of oil and natural gas, triggered by oil embargoes and a slowdown in the development of nuclear power.

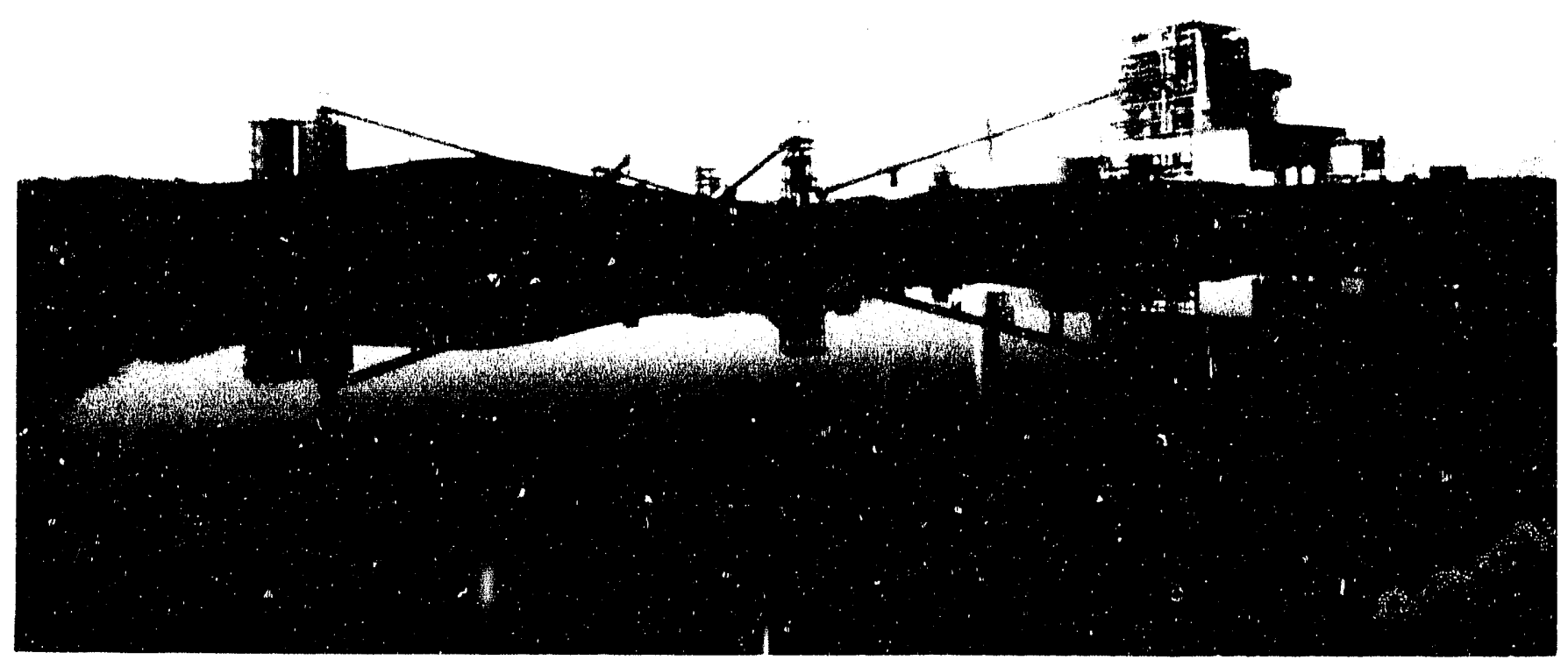

L.S. coal production was spurred to record levels in the 1980 's by a growing demand for electricity. This power plant in Louisiana is one of nearly 500 power plants in the Enited States that generate electricity from coal. 
Both total electricity generation and coal-generated electricity rose almost steadily in the decade of the 1980's. Total electricity generation grew from 2,286 billion kilowatthours in 1980 to 2,781 billion kilowatthours in 1989, increasing at an average annual rate of over 2 percent and setting new records in all but 1 year. At the same time, coal-generated ciectricity rose from 1,162 billion kilowatthours to 1,552 billion kilowatthours, growing at a faster annual average rate of over 3 percent and reaching record levels in all but 2 years of the decade. As a result, coal's share of electricity generation increased from 51 percent in 1980 to 56 percent in 1989. This is reflected in the substantially larger amount of coal consumed by utilities: 766 million short tons in 1989 as compared with 569 million short tons in 1980. Virtually all of this coal was produced from U.S. coal mines; less than 1 percent was imported.

By the end of the decade, nine States relied on coal for over three-fourths of their generating capability. An additional 14 States depended on coal for 50 10 70 percent of their electricity generation.

Because of its strong relationship with electricity generation, the trend of coal product. In in the 1980 's was very closely aligned with that of utility coal consump tion, except for a promounced drop in 1983. That anom aly was the only time in the decade when annual production fell below $8(x)$ million short tons. It stemmed from the fact that coal consumption was down in 1982 , largely because of an economic recession. Consequently, consumers carried large coal stockpiles into 198.3, when the stocks were drawn down at the expense of mine production. A decline in the coal export market in 1983 also curtailed coal production.

The amount of coal produced for consumers other' than electric utilities was comparatively small, but it helped bolster the level of production in the 1980's. Generally, about 14 percent of the coal produced was used by various manufacturing industries, ranging from coke and coal gasification plants to cement plants and paper mills. I.ess than 1 percent went to residential and commercial consumers. Over 10 percent of the annual coal output was for the export market, where the United States ranked as the world's leading coal exporter until 1984, when Australia gained first place. Those coal exports, valued at $\$ 4$ to $\$ 6$ billion annually, helped the Nation's balance of payments.

Table FE1. U.S. Coal Production and Related Statistics, 1980-1989

\begin{tabular}{|c|c|c|c|c|c|c|c|c|c|c|}
\hline & 1980 & 1981 & 1982 & 1983 & 1984 & 1985 & 1986 & 1987 & 1988 & 1989 \\
\hline \multicolumn{11}{|l|}{ Production (million short tons) } \\
\hline Total ...................................... & 830 & 824 & 838 & 782 & 896 & 884 & 890 & 919 & 950 & 981 \\
\hline \multicolumn{11}{|l|}{ Mining Method } \\
\hline 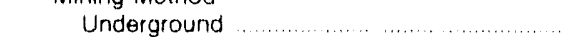 & 338 & 317 & 330 & 300 & 352 & 351 & 360 & 373 & 382 & 394 \\
\hline Surface & 492 & 507 & 499 & 482 & 544 & 533 & 530 & 546 & 568 & 587 \\
\hline \multicolumn{11}{|l|}{ Region } \\
\hline Appalachian & 444 & 433 & 433 & 383 & 444 & 427 & 429 & 443 & 449 & 464 \\
\hline Interior $\ldots \ldots \ldots \ldots \ldots \ldots$ & 176 & 167 & 178 & 1,74 & 198 & 188 & 197 & 202 & 193 & 198 \\
\hline 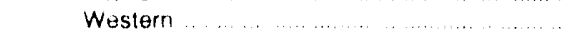 & 209 & 224 & 227 & 225 & 254 & 269 & 265 & 274 & 308 & 318 \\
\hline \multicolumn{11}{|l|}{ Federally Administered Lands } \\
\hline Total & 93 & 138 & 130 & 124 & 136 & 184 & 190 & 195 & 225 & 236 \\
\hline Federal Coal Leases & 69 & 116 & 101 & 99 & 116 & 159 & 167 & 171 & 199 & 209 \\
\hline Indian Coal Lease & 2.4 & 22 & 29 & 25 & 20 & 25 & 23 & 24 & 26 & 27 \\
\hline \multicolumn{11}{|l|}{ Coal Rank } \\
\hline Anthracite & 6 & 5 & 5 & 4 & 4 & 5 & 4 & 4 & 4 & 3 \\
\hline Bituminous & 629 & 608 & 620 & 569 & 650 & 614 & 620 & 637 & 638 & 660 \\
\hline Subbetuminous & 148 & 160 & 161 & 151 & 179 & 193 & 190 & 200 & 224 & 231 \\
\hline Lignite & 47 & 51 & 52 & 58 & 63 & 72 & 76 & 78 & 85 & 86 \\
\hline \multicolumn{11}{|l|}{ Average Value (dollars per short ton) } \\
\hline Total & 2465 & 26.40 & 27.25 & 25.98 & 25.61 & 25.20 & 23.79 & 23.07 & 22.07 & 21.82 \\
\hline Undergroulid .... & 33.50 & 3578 & 35.78 & 34.47 & 33.36 & 32.91 & 30.33 & 29.63 & 28.97 & 28.44 \\
\hline Surface $\quad \ldots \quad \ldots \ldots$ & 18.78 & 20.60 & 21.46 & 20.68 & 20.59 & 20.13 & 19.34 & 18.58 & 17.43 & 17.38 \\
\hline \multicolumn{11}{|l|}{ Number of Mines" } \\
\hline Total ............ & 3,969 & 4,140 & 4,098 & 3,405 & 3,566 & 3,355 & 3,175 & 3,030 & 2,915 & 2,821 \\
\hline \multicolumn{11}{|l|}{ Mining Method } \\
\hline$\ldots \ldots \ldots \ldots$ & 1,887 & 2.020 & 1.991 & 1,638 & 1,763 & 1,695 & 1,640 & 1,563 & 1,463 & 1,426 \\
\hline Surface & 2,082 & 2.120 & 2,107 & 1,167 & 1,803 & 1,660 & 1,535 & 1,467 & 1452 & 1,395 \\
\hline \multicolumn{11}{|l|}{ Region } \\
\hline Appalachıarı & 3,498 & 3,641 & 3,592 & 2,971 & 3,141 & 2,962 & 2,809 & 2.672 & 2,563 & 2,484 \\
\hline$\left(\begin{array}{l}1 \\
\ldots \ldots\end{array}\right.$ & 337 & 365 & 372 & 311 & 303 & 278 & 256 & 255 & 248 & 235 \\
\hline 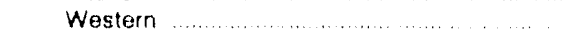 & 134 & 134 & 134 & 12.3 & 122 & 115 & 110 & 103 & 104 & 102 \\
\hline \multicolumn{11}{|l|}{ Large Mines ${ }^{\circ}$} \\
\hline Total .............. & 323 & 299 & 322 & 294 & 337 & 343 & 350 & 363 & 349 & 359 \\
\hline Underground & 170 & 156 & 169 & 154 & 179 & 186 & 186 & 188 & 181 & 190 \\
\hline Surface & 153 & 143 & $i 53$ & 140 & 158 & 157 & $i 64$ & 175 & 168 & 169 \\
\hline Percentage of Production & 60) & 57 & 61 & 65 & 65 & 67 & 69 & 71 & 72 & 73 \\
\hline Longwall Mining Installations .... & NA & NA & 112 & 118 & 112 & 108 & 101 & 102 & 92 & 95 \\
\hline
\end{tabular}

See footnotes at end of the table 
Table FE1. U.S. Coal Production and Related Statistics, 1980-1989-Continued

\begin{tabular}{|c|c|c|c|c|c|c|c|c|c|c|}
\hline & 1980 & 1981 & 1982 & 1983 & 1984 & 1985 & 1986 & 1987 & 1988 & 1989 \\
\hline \multicolumn{11}{|l|}{ Employment (thoussands) ${ }^{\mathrm{C}}$} \\
\hline $\begin{array}{l}\text { Total ............................. } \\
\text { Mining Method }\end{array}$ & 229 & 229 & 217 & 176 & 178 & 169 & 155 & 143 & 135 & 131 \\
\hline \multicolumn{11}{|l|}{ Mining Method } \\
\hline 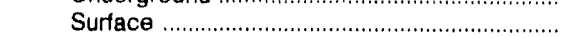 & $\begin{array}{r}151 \\
78\end{array}$ & $\begin{array}{r}152 \\
78\end{array}$ & $\begin{array}{r}147 \\
76\end{array}$ & $\begin{array}{r}712 \\
64\end{array}$ & $\begin{array}{r}112 \\
66\end{array}$ & $\begin{array}{r}107 \\
62\end{array}$ & $\begin{array}{l}98 \\
56\end{array}$ & $\begin{array}{l}90 \\
53\end{array}$ & $\begin{array}{l}85 \\
51\end{array}$ & $\begin{array}{l}84 \\
48\end{array}$ \\
\hline \multicolumn{11}{|l|}{ Region } \\
\hline Äppalachian & 171 & 172 & 162 & 126 & 130 & 122 & 111 & 102 & 97 & 96 \\
\hline 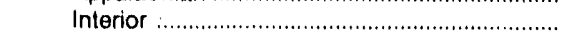 & 40 & 39 & 37 & 35 & 32 & 32 & 29 & 28 & 25 & 23 \\
\hline Western & 18 & 19 & 17 & 15 & 16 & 15 & 14 & 13 & 13 & 12 \\
\hline \multicolumn{11}{|l|}{ Productivity (short tons per miner hour) } \\
\hline Total & 1.9 & 2.1 & 2.1 & 2.5 & 2.6 & 2.7 & 3.0 & 3.3 & 3.6 & 3.7 \\
\hline \multicolumn{11}{|l|}{ Mining Method } \\
\hline Underground & 1.2 & 1.3 & 1.4 & 1.6 & 1.7 & 1.8 & 2.0 & 2.2 & 2.4 & 2.5 \\
\hline Surface & 3.2 & 3.4 & 3.4 & 3.8 & 4.0 & 4.2 & 4.6 & 5.0 & 5.3 & 5.6 \\
\hline \multicolumn{11}{|l|}{ Rer on } \\
\hline Appalachian & 1.4 & 1.5 & 1.5 & 1.8 & 1.9 & 1.9 & 2.1 & 2.3 & 2.4 & 2.5 \\
\hline Interior & 2.3 & 2.4 & 2.4 & 2.7 & 2.8 & 2.8 & 3.1 & 3.3 & 3.5 & 3.8 \\
\hline Western & 5.6 & 6.2 & 6.3 & 7.6 & 8.3 & 8.6 & 9.3 & 10.4 & 11.0 & 11.6 \\
\hline Fatalities & 133 & 153 & 122 & 70 & 125 & 68 & 89 & 63 & 52 & 62 \\
\hline \multirow{2}{*}{\multicolumn{11}{|c|}{ Average Coal Quallty }} \\
\hline \multicolumn{8}{|l|}{ Production } & & & \\
\hline Heat Content (million Btu per short ton) ............... & 22.4 & 22.3 & 22.2 & 22.1 & 22.0 & 21.9 & 21.9 & 21.9 & 21.8 & 21.8 \\
\hline \multicolumn{11}{|l|}{ Utility Coal } \\
\hline $\begin{array}{l}\text { Heat Content (million Btu per short ton) ........... } \\
\text { Sulfur Content (percentage by weight) }\end{array}$ & 21.3 & 21.1 & 2.1 .2 & 21.1 & 21.1 & 21.0 & 21.1 & 21.1 & 20.9 & 20.8 \\
\hline $\begin{array}{l}\text { Sulfur Content (percentage by weight) ................ } \\
\text { Ash Content (percentage by weight) }\end{array}$ & 1.6 & 1.5 & 1.5 & 1.5 & 1.5 & 1.4 & 1.4 & 1.4 & 1.3 & 1.3 \\
\hline Ash Content (percentage by weight) ................... & 11.1 & 11.1 & 10.7 & 10,4 & 10.4 & 10.0 & 10.0 & 10.0 & 9.9 & 9.9 \\
\hline \multirow{2}{*}{\multicolumn{11}{|c|}{$\begin{array}{l}\text { Coal Dlsposition (million short tons) } \\
\text { Domestic Consumption }\end{array}$}} \\
\hline & & & & & & & & & & \\
\hline Total & 703 & 733 & 707 & 737 & 791 & 818 & 804 & 837 & 884 & 829 \\
\hline 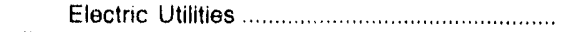 & 569 & 597 & 594 & 625 & 664 & 694 & 685 & 718 & 758 & 766 \\
\hline Exports & 92 & 113 & 106 & 18 & 81 & 93 & 86 & 80 & 95 & 101 \\
\hline \multicolumn{11}{|c|}{$\begin{array}{l}\text { - Annual production over } 10,000 \text { short tons each. } \\
\text { b Annual production over } 500,000 \text { shot tons each. } \\
\text { c At mines that produced over } 10,000 \text { short tons per year. } \\
\text { NA = Not Available. } \\
\text { Note: Total may not equal sum of components because of independent rounding. } \\
\text { Sources: Energy Information Administration, Coal Production (various issues); Quarterly Coal Report (various issues); Cost and Quality of Fuels for } \\
\text { Electric Utility Plants (various issues); U.S. Departrnent of the Interior, Minerals Managemint Service, The 1988 Report on Feceipts from Federal and In- } \\
\text { tian Leases, and predecessor annual reports; U.S. Department of Labor. Mine Safety and Health Administration, Injury Experience in Coal Mining (various }\end{array}$} \\
\hline
\end{tabular}

\section{Coal-Producing Regions}

All three coal-protucing regions--the Appalachian, Interior, and Western--contributed to the higher levels of coal production achieved in the 1980's, but to vary. ing degrees. The Appalachian Region retained its historical role as the Nation's major source of coal, supplying about 40 percent of the coal produced during the decade. It was the principal source of bituminous coal, including coking coal, and anthracite. Production from the region averaged over 400 million short tons per year, reaching a record 464 million short tons at the end of the decade. Although most of Appalachia's coal was shipped to domestic power plants, it also supplied about 90 percent of the coal exported.

Coal production from the several basins composing the Interior Region rose from an annual average of less than 180 million short tons in the first half of the 1980 's to nearly 200 million short tons in the second half. Most of the increase reflected thc growing use of lignite mined in Texas and consumed by power plants located near the mines. The demand for bituminous coal, mined chiefly in the northern part of the region, was hampered by the coal's generally high sulfur conten.

Of the three coal-producing regions, the Western Region not only accounted for most of the additional coal produced in the $1980^{\prime} \mathrm{s}$, but it was also the fastest growing. Rising from over 200 million short tons in 1980 to over 300 million short tons in 1989, the West's share of total coal production advanced from 25 percent at the beginning of the decade to 32 percent at the end. Low-sulfur subbituninous coal accounted for most of the output from this region, which consists of scattered coal basins. Domestic electric power plants were the principal markets for Western coal, although some of it was also exported. 


\section{Coal-Producing States}

During the decade of the 1980's, record amounts of coal were produced in 16 of the 27 coal-producing States. More than half of these peaks in production occurred in western States.

The Nation's top three coal-producing States were Kentucky, Wyoming, and West Virginia. Production from each of these States averaged well over 100 million short tons annually, with their combined tonnage accounting for over two-fifths of the national total. Kentucky led the Nation until Wyoming gained first place in 1988. West Virginia was third-largest throughout the decade. However, based on the energy content and value of the coal produced, both Kentucky and West Virginia ranked above Wyoming. This was because Wyomitig's coal is nearly all subbituminous coal, which has a lower heat content and mine price than the bituminous coal produced in Kentucky and West Virginia. Yet, in other aspects, coal mining in Wyoming can be described with superlatives. Wyoming was the source of about half of the coal produced in the West, and it had the Nation's thickest coalbeds (averaging over 70 feet), the largest and most productive surface coal mines, and vast reserves of low-sulfur coal.

Several of the other coal-producing States were notable for different reasons. Louisiana became a coal producer in 1985, when a lignite surface mine was opened to supply a power plant constructed near the mine. Texas retained its commanding position as the Nation's leading producer of lignite by nearly doubling its annual output from 29 million short tons in 1980 to 54 million short tons in 1989, all consumed by power plants in the State.

In the anthracite region of northeastern Pennsylvania, an area that once knew a large mining industry, annual production dropped to less than 4 million short tons as the decade progressed. In Alaska, by contrast, the output from the State's only coal mine nearly doubled, totaling over 1 million short tons, with the beginning of coal expor:s to Korea in 1985.

Arizona remained the only State with a coal-slurry pipeline, the 273-mile-long, 18-inch-wide Black Mesa line. It carried over 4 million short tons of tituminous coal annually from a mine in northeastern Arizona to a power plant in southern Nevada. Alabama and New Mexicu led the Nation in the production of coalbed methane, which supplements the supply of natural gas. In California, a single small mine produced lignite solely for its content of montan wax, the only commercial U.S. source of this industrial wax. In another novel use of coal, resin was extracted from some of the bituminous coal produced in Utah.

\section{Table FE2. Peak Year of Coal Production by State, from Earliest Records Through 1989}

\begin{tabular}{|c|c|c|}
\hline State & Year & $\begin{array}{c}\text { Quantity } \\
\text { (thousand short } \\
\text { tons) }\end{array}$ \\
\hline Alabama & 1989 & 27,992 \\
\hline 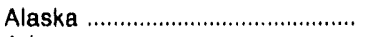 & 1988 & 1,745 \\
\hline 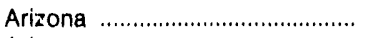 & 1988 & 12,398 \\
\hline 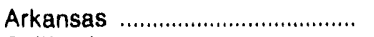 & 1907 & 2,670 \\
\hline 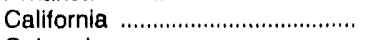 & 1880 & 237 \\
\hline 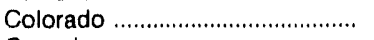 & 1981 & 19,897 \\
\hline 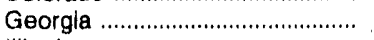 & 1903 & 417 \\
\hline 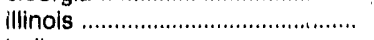 & 1918 & 89,291 \\
\hline 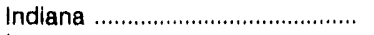 & 1984 & 37,555 \\
\hline 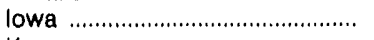 & 1917 & 8,966 \\
\hline 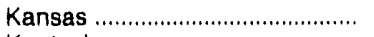 & 1918 & 7,562 \\
\hline 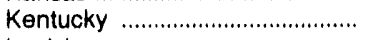 & 1989 & 167,389 \\
\hline 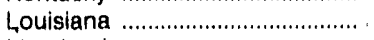 & 1989 & 2,983 \\
\hline 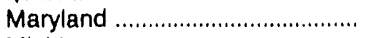 & 1907 & 5,533 \\
\hline 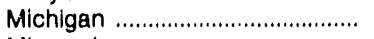 & 1907 & 2,036 \\
\hline 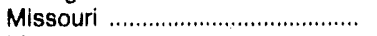 & 1984 & 6,733 \\
\hline 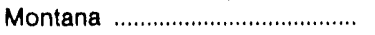 & 1988 & 38,881 \\
\hline New Mexico ..................................... & 1989 & 23,702 \\
\hline 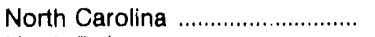 & 1922 & 79 \\
\hline 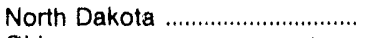 & 1988 & 29,731 \\
\hline Ohio & 1970 & 55,351 \\
\hline 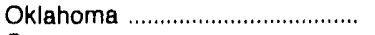 & 1978 & 6,070 \\
\hline 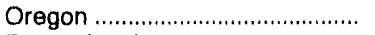 & 1904 & 112 \\
\hline 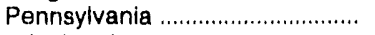 & 1918 & 277,377 \\
\hline 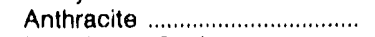 & 1917 & 99,612 \\
\hline Bituminous Coal ......................... & 1918 & 178,551 \\
\hline 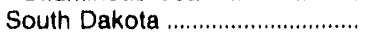 & 1941 & 71 \\
\hline 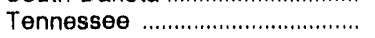 & 1972 & 11,260 \\
\hline 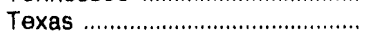 & 1989 & 53,854 \\
\hline 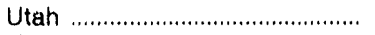 & 1989 & 20,102 \\
\hline 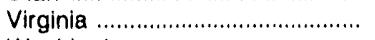 & 1988 & 45,886 \\
\hline 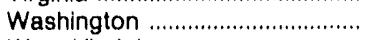 & 1988 & 5,170 \\
\hline 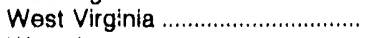 & 1947 & 176,157 \\
\hline 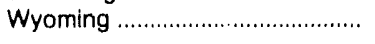 & 1989 & 171,558 \\
\hline 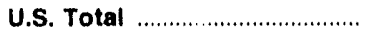 & 1989 & 980,729 \\
\hline
\end{tabular}

Note: In the 1980s, no coal was produced in Michigan, North Carolina, Oregon, or South Dakota. Georgia had no coal production after 1984.

Source: Bureau of Mines, U.S. Department of the Interior, Minerals Yearbook, and Energy Information Administration, Coal Produc. tion, various issues.

\section{Federal and Indian Coal}

During the 1980's, the percentage of the Nation's coal output from Federal and Indian lands was larger than in previous decades. Production from these lands rose from 93 million short tons in 1980 to 236 million short tons in 1989. Their share of total production increased from 11 to 24 percent. During the period, royalties from this production totaled about $\$ 990$ million from Federal coal leases and about $\$ 220$ million from Indian coal leases.

About 70 percent of the coal from Federal leases was produced in Wyoming. All of the other coal mined from Federal leases was confined to Western Siates, except for a very small amount from Alabama and Kentucky. Indian coal reserves were leased in Arizona, New Mexico, and Montana. At the end of 1989, coal 
leases totaled about 236,000 acres on Federal !and and 156,000 acres on Indian land.

\section{Mines}

During the decade, the number of active coal mines that annually produced over 10,000 short tons each dropped fiom about 4,000 in the early 1980's to fewer than 3,000 in 1989. (Another 1,000 or more smaller mines were also active, but their combined output was only about 1 percent of the total.) About half of the mines were surface operations and half underground. Nationally, surface mines produced about 60 percent of the coal. But on a regional basis the amount of surface-mined coal increased westwards, from 40 percent in the Appalachian Region, to 60 percent in the Interior Region, and 90 percent in the Western Region.

Nearly 90 percent of the active mines were in the Appalachian Region. With most of the mine closings also occurring in this region, the number of active Appalachian coal mines fell from about 3,500 to 2,500 over the decade. By comparison, the decrease in the other regions was smaller. The number of active mines dropped from 337 to 235 in the Interior Region and from 134 to 102 in the Western Region.

In about half of the coal-producing States, coal was mined by both surface and underground mines. In six western States production was only from surface mines. Utah had the distinction of having only underground coal mines.

Although the total number of mines dropped over the decade, more coal was produced than ever before because there were more large mines, defined as those producing over 500,000 short tons annually. At the beginning of the decade, large mines accounted for 8 percent of the total number of mines and held a $6 \mathrm{C}$-percent share of the coal produced. At the end of the decade, they accounted for 13 percent of the total number and provided over 70 percent of production. The trend to larger mines, which began in earlier decades, was made possible by greater mechanization, the use of large-capacity equipment, and, especially in the West, access to large blocks of coal to form mining units.

The biggest coal mines were surface mines, several of which produced more than 10 million short tons per year. Most of these large operations were in the sparsely populated, generally flat areas of the West, where they had the advantage of working thick coalbeds that are covered by relatively' thin overburden. The largest coal mine, both in the Nation and North America, was the Black Thunder mine in Wyoming's Powder River Basin. In 1989, this mine alone produced about 30 mil- lion short tons, an amount greater than the output of 17 smaller coal-producing States. The largest underground mines, located in the Appalachian and Interior regions, produced as much as 5 million short tons per year.

\section{Employment}

The record-breaking levels of coal production in the 1980 's were achieved with a work force that became smaller as the decade progressed. It decreased by more than 40 percent during the period, from nearly 230,000 at the beginning to 131,000 at the end. For the decade, an average of 176,000 miners were employed annually in the coal industry. Only in the 1960's was the average level of employ ment lower, at 150,000, but those miners produced 40 percent less coal.

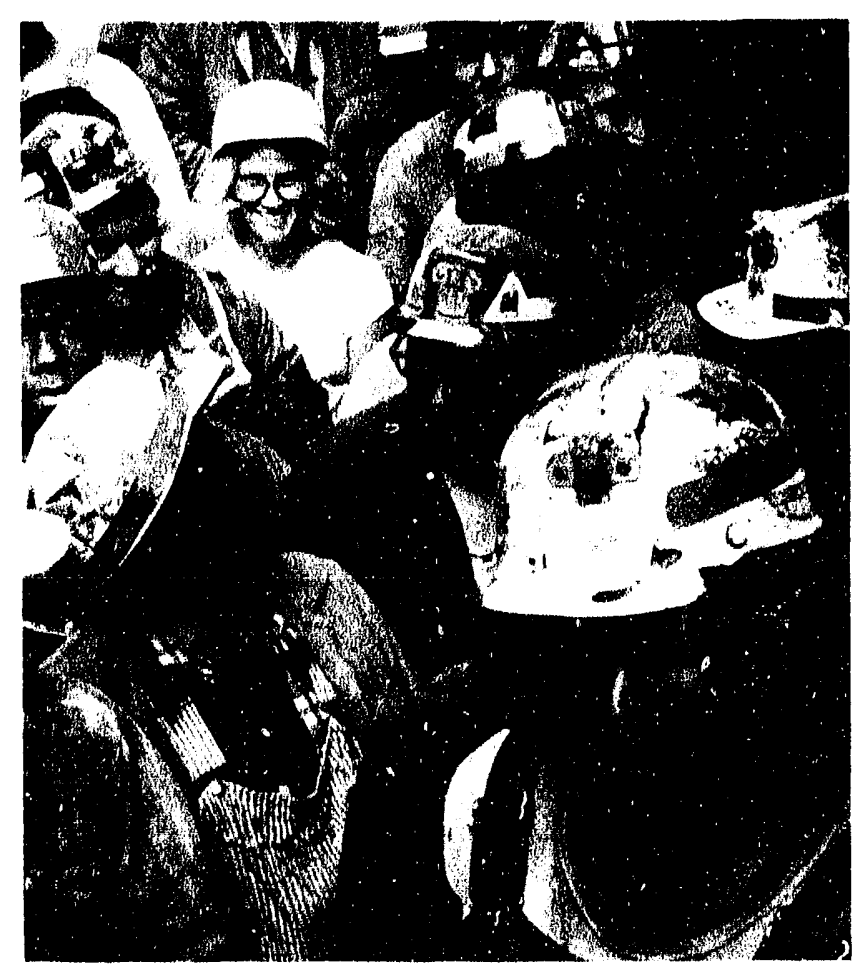

The record amounts of coal produced in the 1980's reflect the efforts of miners like these. Although the size of the work force dropped during the decade, productivity rose.

The decreased number of coal miners needed in the 1980 's reflects not only the continuing advances made in mechanizing nearly all phases of mining but also an increase in surface mining, which requires fewer workers. Compared with 1980, about 67,000 fewer miners were employed at underground mines in 1989, a drop of over 40 percent. At surface mines the labor force decreased by about 30,000, or nearly 40 percent. 
The coal mining force in the Appalachian Region, with over 70 percent of the Nation's coal miners, experienced the greatest loss of jobs during the $1980^{\prime} \mathrm{s}$. Coal mine employment in the region fell from more than 170,000 at the start of the decade to less than 100,000 at the end. The loss of coal-mining jobs was less severe in other regions, falling from 40,000 to 23,000 in the Interior Region and from 18,000 to 12,000 in the Western Region.

According to a survey of the coal mining workforce made by the U.S. Bureau of Mines, the average miner in 1986 was 39 years old and had 11 years of coal mining experience. He or she had been with the same company for 8 years and in the same job for half of the time. Over three-fourths of the miners had a high school or better education. More than 3,000 women were employed in production work at coal mines, a slightly larger number than those with office jobs.

\section{Productivity}

While coal mine employment dropped sharply in the 1980 's, productivity made decidely strong gains. Miners produced coal at a higher rate at both underground and surface mines in all regions. Rising each year, their average hourly productivity nearly doubled as it increased from 1.9 short tons in 1980 to a record 3.7 short tons in 1989. Productivity rose from 1.2 to 2.5 short tons at underground mines, and from 3.2 to 5.6 short tons at surface mines.

This dramatic rise in productivity can be attributed to several factors. Beginning with technology, efficiency was improved through the use of increased haulage capacity and better mining equipment. Longwall mining systems became more productive because many worked in larger panels of coal, containing as much as 1 million short tons, that enabled more coal to be produced before downtime was needed to move the equipment to a new panel. Some longwall systems were equipped with electrohydraulic roof supports, which can be moved faster by fewer miners.

The increase in productivity also reflects the successful adaptation of mining practices with the health and safety standards and reclamation laws enacted in the 1960 's and 1970's. Initially, these regulations interfered with productivity, diverting some workers and equipment from production activities. Some new safety procedures had to be incorporated into mining practices, and reclamation work had to become a part of surface mining operations. The stricter safety standards led to a better safety record in the 1980 's. Total coal miner fatalities in the decade numbered less than 1,000$)$, compared with 1,500 in the 1970 's.
Figure FE1. Trends in Coal Production and Demand in the 1980's

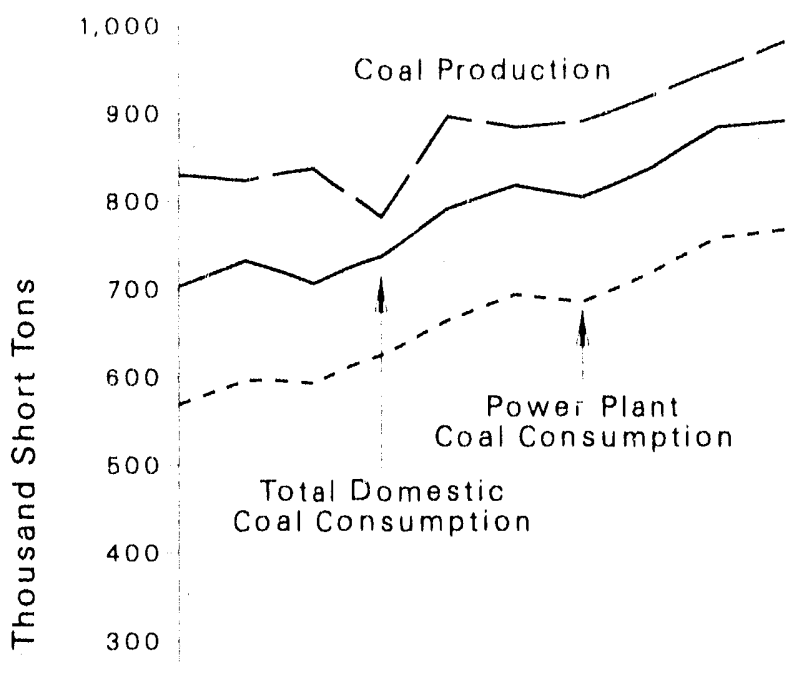

200

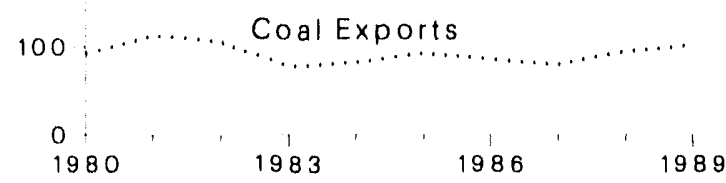

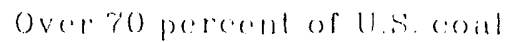

produced in the logo's was nesed

hy domestice power plants, which

reliced on conal for hide of lhe

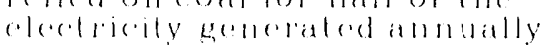

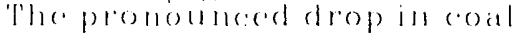

ollpul in logs:s was lhe coll

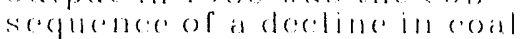

comsinmplion in logse, which les.s

nlled in a drawalown of lanker

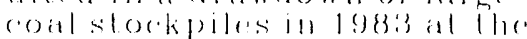

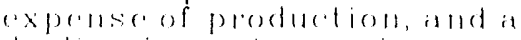

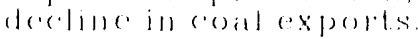

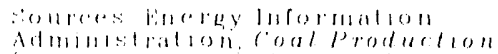

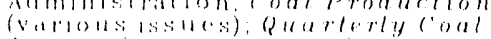

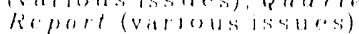

Gains in overall productivity can also be credited to the closing of small, less efficient coal mines, which shifted a larger share of production to larger, more efficient mines. The large mines in the West, which produce from thick coalbeds, contributed significantly to the greater productivity in surface mining.

Still another factor that had a fa /orable effect on productivity was the decrease in major strikes during the 1980 's. The only major strike of the United Mine Workers of America (UMWA) occurred in 1981, closing many mines for 72 days. By contrast, in the 1970 's, the UMWA walked out three times, causing a loss of over 180 workdays. Of the several local strikes in the 1980's, the dispute between the UMWA and the Pittston Coal Group in 1989 attracted the most attention. 


\section{Major Coal Companies}

Of the numerous companies that produced coal in the 1980 's, the top three were the Peabody Holding Company, Consolidation Coal Company, and Amax Coal Company. Their combined share of production averaged nearly one-fifill of the tctal. In the late 1980's, Peabody produced over 80 millinn short tons annually from mines in 10 States. Consolidation Coal, operating in 8 States, averaged over 50 million short tons per year. Amax averaged about 37 million short tons annually from mines in 6 States.

\section{Coalbeds}

Coal was mised from more than 300 coalbeds in the 1980 's. The greatest number of coalpeds mined w as in the Appalachian Region, with over 60 mined in West Virginia alone. However, about one-third of U.S. coal production was from only four coalbeds: the Wyodak, Pittsburgh, No. 6, and No. 9. It is estimated that these four coalbeds contain approximately one-fourth of the U.S. demonstrated coal reserve base of 473 billion short tons.

The Wyodak is a bed of low-sulfur subbituminous coal located in the Powder River Basin of northeastern Wyoming. Averaging 70 feet in thickness, it is the thickest coalbed mined in the United States, and in places yields about 100,000 short tons of coal per acre. Because of the increased mining in the Powder River Basin, the

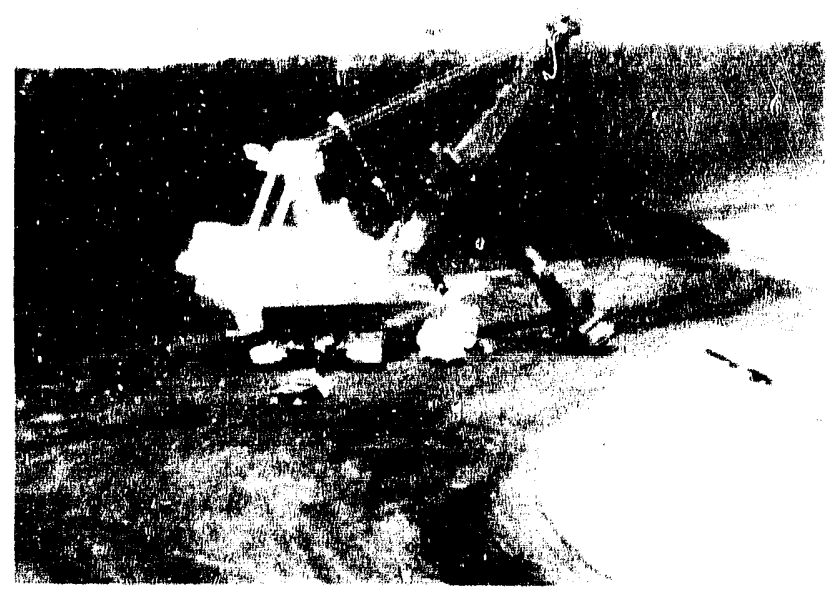

The Nation's thickest coalbeds are in Wyoming's Pow'der River Basin. The Wyodak coalbed shown here is over 70 feet thick, has a low' sulfur content, and is economical to mine. amount of coal extracted from the Wyodak more than tripled during the decarle, from 40 million short tons in 1980 to 143 million short tons in 1989.

The Pittsburgh is a bed of bituminous coal averaging 5 to 6 feet in thickness and underlying the northern part of the Appalachian Region. Its sulfur content generally is over 1 percent, by weight. Contrasting with the relatively recent mining of the Wyodak, the Pittsburgh was one of the first coalbeds mined in the United States. Because its coal helped fuel early industrial development, the Pittsburgh coalbed has been acclaimed as the mo'st important coalbed in the Nation. An average of 55 million short tons were produced annually from this bed during the decade.

Both the No. 6 and No. 9 coalbeds, which averege about 6 feet in thickness, are in the Illinois Basin, in the northern part of the Interior Region. The coal in: both beds is bituminous in rank and has a relatively tigh sulfur content, gene ally over 3 percent by weight. Some 40 to 50 million short tons of coal were mined annually from these beds.

\section{Coal Rank}

All ranks of coal, except anthracite, were produced in larger quantities during the 1980 's. Bituminous coal production, centered in the Appalachian Region, continued to predominate with an average of over 600 million short tons mined annually. However, its share of the total output decreased from three-fourths in 1980 to two thirds by 1989. This was due mostly to substantial increases in production of subbituminous coal and, to a lesser extent, lignite. The steadily rising output of these low-rank coals stems from the largescale development that began in the 1970's, when their low surface-mining costs and low sulfur content made them economically and environmentally attractive fuels for power plants.

Subbituminous coal was produced only in the Western Region, chiefly in Wyorning. During the decade, the output of this coal rose almost steadily, from $148 \mathrm{mil}$ lion short tons in 1980 to 231 million short tons in 1989. Although lignite was also produced in the Western Region, its greatest development was in the Interior Region, specifically in Texas. Total lignite production during the period nearly doubled, from 77 million short tons to 86 million short tons. In striking contrast, anthracite production continued to wane, dropping from 6 million short tons in 1980 to 3 million short tons in 1989. The contrasting production trends for lignite and anthracite are underscored by the facts that in 1970, lignite production was only 8 millic short tons, and that before 1950), anthracite production was over 40 million short tons annually. 


\section{Heat Content}

Although coal production rose in the 1980's, the average heat content of the output declined because of a faster increase in output of low-rank coals, which have a lower heat content. Consequently, the average heat content of total production decreased from 22.4 million Btu per short ton in 1980 to 21.8 million Btu per short ton in 1989. Most of the impact of this was at power plants, where the heat content of the coal consumed dropped from 21.3 million Btu per short ton in 1980 to 20.8 million Btu per short ton in 1989. By comparison, the heat content of coal for other consumers and the coal exported remained relatively stable. Although the averuge heat content of coal declined, the total energy content of the coal produced rose markedly over the decade, from 18.6 quadrillion Btu in 1980 to 21.3 quadrillion Btu in 1989, because of the increaced tonnage.

\section{Coal Quality}

Unquestionably, coal output in the 1980 's played a vital role in the Nation's economy. But coal is an imperfect fuel, with environmiental drawbacks due to its natural composition. Of foremost concern is the sulfur present in coal and the emissions of sulfur dioxide formed when coal is burned. This gas and the nitrogen oxides also produced during combustion are converted to sulfur and nitric acids, which can lead to the formation of acid rain. Other pollutants are particulates, derived from incombustible material in coal and com. posed mostly of silica and alumina. Sulfur dioxide emissions from power plants are controlled by flue gas scrubbers. Emissions of nitrogen oxides are usually controlled by reducing the amount of air during combustion and by lowering combustion temperatures. Particulates are generally removed by electrostatic precipitators. In the 1980 's, research and development was underway to improve the performance of these techniques and to produce innovative nonpolluting technology for generating electricity from high-sulfur coal.

The quality of coal delivered to power plants provides a measure of the quality of coal consumed over the decade. In general, it has improved. The sulfur content, by weight, declined from 1.6 percent in 1980 to 1.3 percent in 1989. The ash content dropped from 11.1 percent to 9.9 percent.

That air quality controls have been effective is shown oy studies made by the Environmental Protection Agency. Data for 1980-1988, the most recent available, show that emissions of sulfur oxides from coal-fired power plants dropped by 8 percent even though 33 percent more coai was consumed. The decrease was largely attributable to a greater number of power plants equipped with scrubbers and meeting new air quality standards, and a reduction in the average sulfur content of the coal consumed.

\section{Price}

The average mine price per short ton of coal declined during the 1980 's, falling from a peak of $\$ 27$ in 1982 to about $\$ 22$ in 1989. The trend was influenced by com. petition within the industry, economic conditions, and the heat content of the coal produced. Taking into account the change in heat content and eliminating the effects of inflation, the real minemouth price of coal fell by abou: one-third during the 1980's.

The price of underground-mined coal ranged from a high of $\$ 36$ per short ton in 1981 and 1982 to a low of $\$ 28$ per short ton in 1989. A ton of surface-mined coal, by contrast, was priced at $\$ 21$ in 1982 and $\$ 17$ in 1989.

Appalachian coal was the highest priced and Western coal the lowest. The difference reflects the facts that Appalachian production was mostly from underground mines, which are more costly to operate, that the bituminous coal produced has a higher heat content, and that the coal markets are nearby.

Western coal was lower priced not only because much of it was produced by large-scale, economica'ly efficient surface mines, but also because it has a lower heat content and requires long-distance transportation to consumers.

\section{Table FE3. U.S. Coal Production, Disposition, and Prices, Selected Years, 1980-2000}

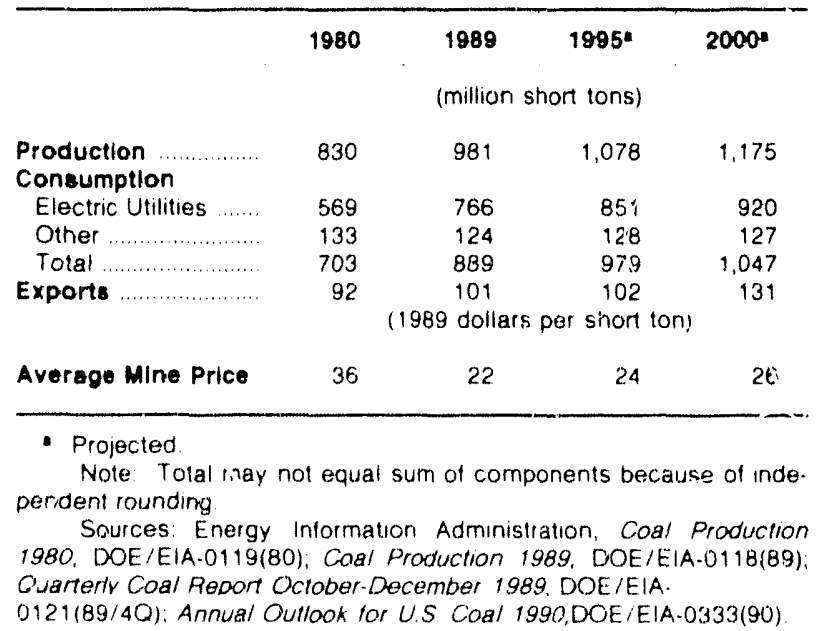




\section{The Next Decade and Beyond}

'The decade of the 1990's will see annual coal production rise above 1 billion short tons. As in the 1980's, most of the coal will be used to generate electricity.

Research and development will go forward into the 1990 's, investigating all parts of the "coal chain," from mine to consumer. Computer-assisted mining systems under development have the potential of aúvancing mining technology through automation, robotics, and networking programs that can monitor a variety of mining activities, resulting in improved health, safety, productivity, reliability, and economy. Progress in coal preparation, mined-land reclamation, as well as in understanding the human factors involved in coal mining, will help shape the future of the coal industry.

Clean coal technology, a term that entered the energy vocabulary in the 1980 's, also holds an important role in creating a bright future for coal. This technology offers a new generation of high-efficiency, cleanburning coal processes. Many of them are more efficient and less costly than conventional processes.

All these efforts are responding to past problems with some of the most sophisticated technology available today. They are aimed at balancing the competing goals of attaining both energy sufficiency and environmental quality from coal.

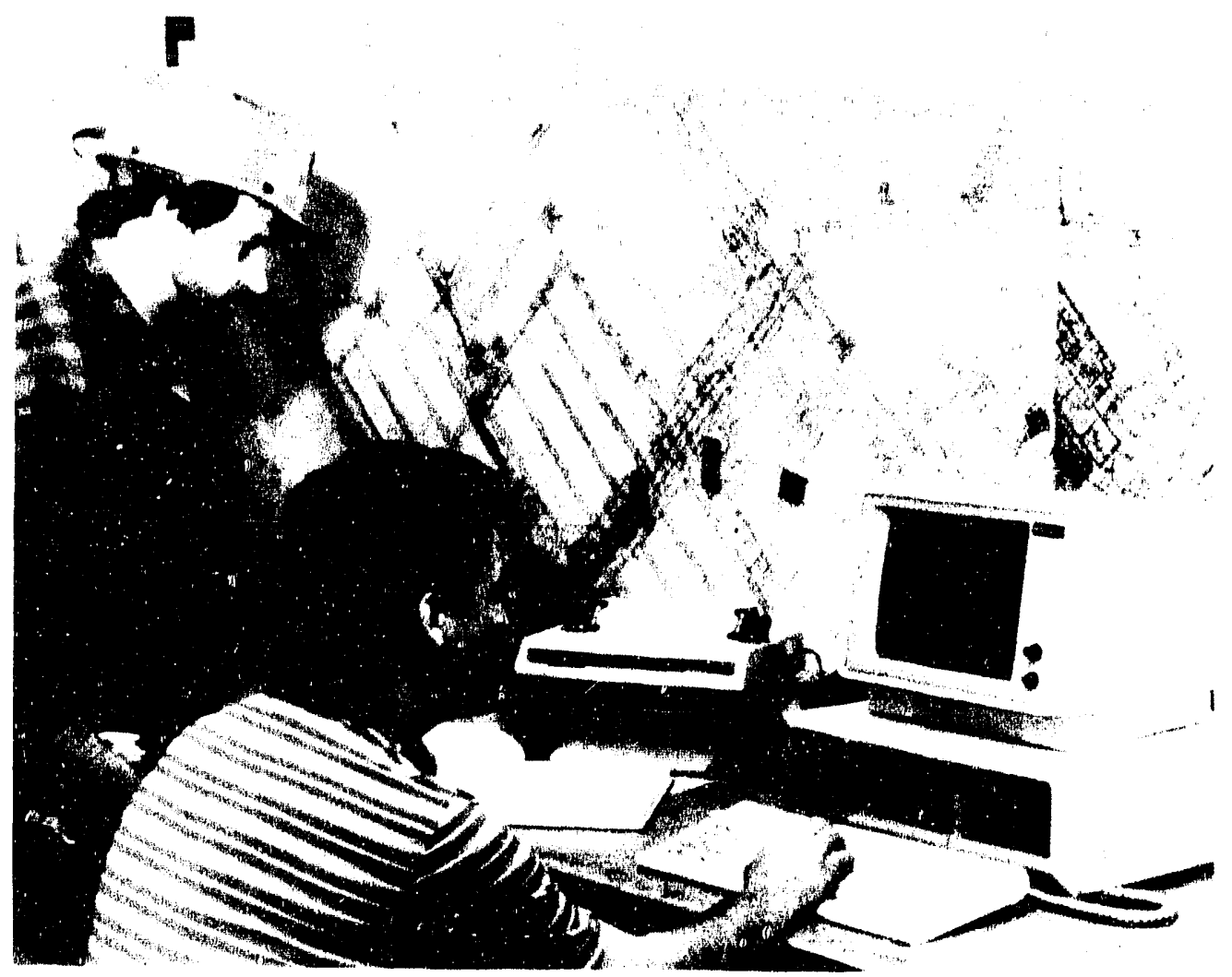

Mining technology is advancing through the use of computers. This computcr is monitoring inethane and coal dust in a mine to detect conditions that could be dangerous to health and safety. 


\section{References}

Coal (and predecessor Coal Mining and Processing). Maclean Hunter Publishing Co. (Chicago, IL.)

Energy Information Administration. Annual Energy Review 1989. DOE/EIA-0384(89). (Washington, DC, May 1990.)

Energy Information Administration. Annual Outlook for U.S. Coal 1990. DOE/EIA-0333(90). (Washington, DC, May 1990.)

Energy Information Administration. Coal Distribution DOE/EIA-0125(89/4Q) and prior issues. (Washington, DC, January-December 1989.)

Energy Information Administration. Coal Production 1989. DOE/EIA-0018(89) and prior issues. (Washington, DC.)

Energy Information Administration. Cost and Quality of Fuels for Electric Utility Plants 1989. DOE/EIA-0191(89) and prior issues. (Washington, DC.)

Energy Information Administration. Electric Power Annual 1988 DOE/EIA-0348(88) and prior issues. (Washington, DC.)

Energy Information Administration. Inventory of Power Plants in the United States 1989. DOE/EIA-0095(89) and prioi issues. (Washington, DC.)

Energy Information Administration. Monthly Energy Review. DOE/EIA-0035. (Washington, DC.)

Energy Information Administration. Quarterly Coal Report October-December 1989. DOE/ EIA-0121(89/4Q) and prior issues. (Washington, DC.)

Keystone Coal Industry Manual. Maclean Hunter Publishing Co. (Chicago, IL.)
Keystone News Bulletin (Monthly). Maclean Hunter Publishing Co. (Chicago, IL.)

Quarterly Review of Methane from Coal Seams Technology. Gas Research Institute. (Golden, CO.)

U.S. Department of Energy, Assistant Secretary for Fossil Energy. Clean Coa! Technology. The New C.oal Era. DOE/FE-0193-P (Washington, DC, Nover ber 1989, revised June 1990.)

U.S. Department of Energy, Assistant Secretary for Fossil Energy. Clean Loal Techno!ogy Demor.stration Program. Annual Report to Congress. DOE. E-0195P. (Washington, DC, March 1990.)

U.S. Department of the Inrsrior, Bureau of Mines, $\mathrm{I}_{\mathrm{n}}$ formation Circular 9192, "Characterization of the 1986 Coal Mining Workforre." (Minneapolis, MN.)

U.S. Departmen: $i$ of the Interior, Bureau of Mines, Minerals Yearbor'k. (Washington, DC.)

U.S. Department of the Interior, Bureau of Mines, Research 88. (Washington, DC.)

U.S. Department of the Interior, Bureau of Mines, Research 89-90. (Washington, DC.)

U.S. Department of the Interior, Minerals Management Service, Royalty Management Program. Mineral Revenues: The 1989 Report on Receipts from Federal and Indian Leases (and prior issues). (Washington, DC.)

U.S. Department of Labor, Mine Safety and Health Administration. Injury Experience in Coal Mining (Annual). (Denver, CO.)

U.S. Environmental Protection Agency. National Air Pollutant Emission Estiriates 1940-1988. EPA-450/4-90-001 (March 1990). (Research Triangle Park, NC.) 


\section{Executive Summary}

\section{Total Coal Production}

Coal production in the United States during 1989 reached a record level of nearly 981 million short tons (Table 1), an increase of about 30 million short tons over 1988. This was the third consecutive year that total production has risen by this amount ( 30 million), and the pattern continued the increased production trend since 1985. Current projections call for continued increases over the next few years.

The principal reason for the increased production was to meet the demand from the coal-fired power plants which generated an all-time record amount of electricity in 1989. Also in 1988, electric utilities had sizeable stock withdrawals, partly to generate more coal-fired electricity to replace hydroelectric power generation during the drought of 1988 . Another reason for increased production was the higher demand for exports of both steam and metallurgical coal to European markets. In response to this higher demand and to offset the decreases in European production, U.S. coal exports increased. One important reason for the increased reliance on U.S. coal, was that one of the other historical suppliers (Australia), experienced labor problems and the amount of their exports to Europe decreased in 1989. Also, the demand for coal in Europe was strong due to new electric plants coming on line and the low rate of exchange of the dollar, which helped make U.S. exports relatively cheaper.

There were 27 States mining coal during the year. Seven States reached record levels of production in 1989: Alabama, Kentucky, Louisiana, New Mexico, Texas, Utah, and Wyoming. The top three State coal producers were Wyoming (172 million short tons), Kentucky (167 million) and West Virginia (154 million). A majority (61 percent) of the Nation's coal was produced in mines East of the Mississippi, but as the larger Western mines continue to produce greater amounts of lo: sulfur subbituminous coal, this majority has and will decrease.

The number of coal mines fell to 3,620, continuing the trend of the last 10 years. During this time smaller mines have been closed due to falling prices and com. petition from larger and more efficient mines.

\section{Survey Highlights}

Tables 2 through 43 in this report include data from mining operations that produced, prepared and processed 10,000 or more short tons during the year. These mines yielded 978 million short tons, or 99.7 percent of the coal production in 1989, and are the concern of the data and analysis below.

- Wyoming led the Nation with 172 million short tons of coal production. This was the second consecutive year that Wyoming was the Nation's top producer. However, Kentucky had the greatest gross increase in tons for the year (about $10 \mathrm{mil}$. lion tons).

- The number of active mines dropped 3 percent from 1988, to 2,821. The greatest decline in mines was in the Appalachian Region, which had 79 fewer mines.

- The average number of miners working daily fell 3 percent this year, to 131,497 workers.

- Productivity reached 3.70 short tons per hour, an all-time record.

- Bituminous coal accounted for over two-thirds of total production, subbituminous coal accounted for about a quarter, lignite, 9 percent, and Pennsylvania anthracite less than 1 percent.

- The average mine price of coal fell for the seventh consecutive year, to $\$ 21.82$ per short ton. 
Table 1. Total U.S. Coal Production ${ }^{1}$ and Number of Mines by State and Type of Mining, 1989

(Thousand Short Tons)

\begin{tabular}{|c|c|c|c|c|c|c|}
\hline \multirow{3}{*}{$\begin{array}{c}\text { Cosi-Producing } \\
\text { State }\end{array}$} & \multicolumn{4}{|c|}{ Type of Mining } & \multicolumn{2}{|c|}{ Total } \\
\hline & \multicolumn{2}{|c|}{ Underground } & \multicolumn{2}{|c|}{ Surface } & \multirow{2}{*}{ Number of Mines } & \multirow{2}{*}{ Production } \\
\hline & Number of Mines & Production & Number of Mines & Production & & \\
\hline 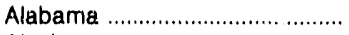 & 15 & 16,327 & 90 & 11,665 & 105 & 27,992 \\
\hline 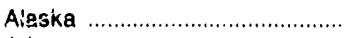 & - & - & 1 & 1,582 & 1 & 1,582 \\
\hline Arizona & - & - & 2 & 11,935 & 2 & 11,935 \\
\hline 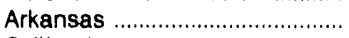 & - & - & 7 & 70 & 7 & 70 \\
\hline California & - & - & 1 & 41 & 1 & 41 \\
\hline 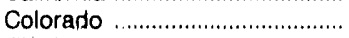 & 16 & 8,511 & 7 & 8,612 & 23 & 17,123 \\
\hline 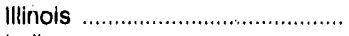 & 30 & 39,337 & 18 & 19,930 & 48 & 59,267 \\
\hline 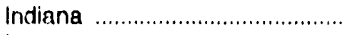 & 6 & 2,519 & 54 & 31,122 & 60 & 33,641 \\
\hline lowa & 1 & 36 & 4 & 394 & 5 & 430 \\
\hline 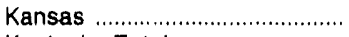 & - & 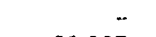 & 5 & 856 & 5 & 856 \\
\hline 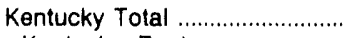 & 670 & 99,067 & 429 & 68,322 & 1,090 & 167,389 \\
\hline Kentucky, Eastern .................... & 644 & 75,746 & 358 & 49,993 & 1,002 & 125,739 \\
\hline Kentucky, Western .................... & 26 & 23,321 & 71 & 18,328 & 97 & 41,649 \\
\hline 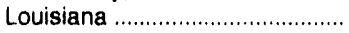 & - & - & 2 & 2.983 & 2 & 2,983 \\
\hline 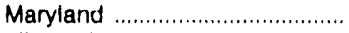 & 2 & 1,842 & 29 & 1,533 & 31 & 3,376 \\
\hline 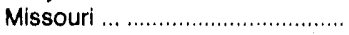 & - & - & 9 & 3,378 & 9 & 3,378 \\
\hline 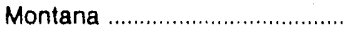 & - & - & 9 & 37,742 & 9 & 37,742 \\
\hline 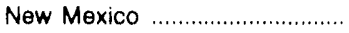 & 1 & 45 & 7 & 23,657 & 8 & 23,702 \\
\hline 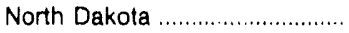 & - & - & 13 & 29.586 & 13 & 29,566 \\
\hline Ohio & 14 & 10,824 & 170 & 22.865 & 184 & 33,689 \\
\hline Oklahoma & 1 & 40 & 20 & 1,713 & 21 & 1,753 \\
\hline 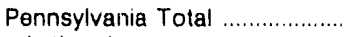 & 156 & 39,607 & 525 & 30,989 & 681 & 70,596 \\
\hline Anthracite $\ldots \ldots \ldots \ldots \ldots \ldots \ldots \ldots \ldots \ldots \ldots \ldots \ldots \ldots \ldots$ & 84 & 513 & 107 & 2,835 & 191 & 3,348 \\
\hline 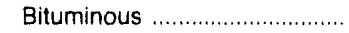 & 72 & 39,095 & 418 & 28,154 & 490 & 67,248 \\
\hline 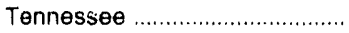 & 68 & 4,688 & 30 & 1,792 & 98 & 6,480 \\
\hline 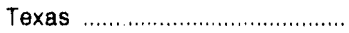 & - & - & 15 & 53,854 & 15 & 53,854 \\
\hline Utah & 21 & 20,102 & - & - & 21 & 20,102 \\
\hline 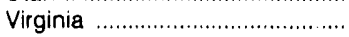 & 271 & 36,044 & 94 & 6,962 & 365 & 43,006 \\
\hline 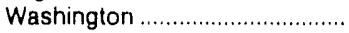 & - & - & 4 & 5,039 & 4 & 5,039 \\
\hline 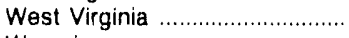 & 461 & 113,202 & 312 & 40,378 & 773 & 153,580 \\
\hline 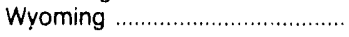 & 4 & 1,645 & 26 & 169,913 & 30 & 171,558 \\
\hline Appalachian Total ${ }^{2} \ldots \ldots \ldots \ldots \ldots$ & 1,631 & 298,279 & 1,608 & 166,178 & 3,239 & 464,457 \\
\hline Interlor Tota| ${ }^{2} \ldots \ldots \ldots \ldots \ldots \ldots \ldots$ & 64 & 65,253 & 205 & 132,627 & 269 & 197,880 \\
\hline Western Total ${ }^{2} \ldots \ldots \ldots \ldots \ldots \ldots \ldots \ldots \ldots \ldots \ldots$ & 42 & 30,304 & 70 & 288,088 & 112 & 318,391 \\
\hline $\begin{array}{l}\text { East of the Miss. River } \\
\text { West of the Miss. River .................. }\end{array}$ & $\begin{array}{r}1,693 \\
44\end{array}$ & $\begin{array}{r}363,456 \\
30,379\end{array}$ & $\begin{array}{r}1,751 \\
132\end{array}$ & $\begin{array}{l}235,559 \\
351,334\end{array}$ & $\begin{array}{r}3,444 \\
176\end{array}$ & $\begin{array}{l}599,015 \\
381,714\end{array}$ \\
\hline U.S. Total & 1,737 & 393,835 & 1,883 & 586,893 & 3,620 & 980,729 \\
\hline
\end{tabular}

1 Excludes silt, culm, refuse bank, slurry dam, and dredge production except for Pennsylvania anthracite.

2 For a definition of coal-producing regions, see Appendix $\mathrm{C}$.

Note: Total may not equal sum of components because of independent rounding.

Source: Energy Intormation Administration, Form EIA-7A, "Coal Production Report."

\section{Production}

Out of 981 million short tons of coal produced in the U.S. in 1989, nearly 978 million short tons of it came from mines that produced a minimum of 10,000 short tons (Table 2). Of that 978 million short tons, 585 million short tons carne from surface mining operations, and 393 million short tons came from underground mines. Virtually all the underground production was concentrated East of the Mississippi, where over 90 percent of the total underground production occurred. While there was a substantial amount of surface production in the East ( 40 percent of total surface production), a small number (47) of surface mines in Wyoming, Texas and Montana accounted for 45 percent of the national surface production. Increased demand for low sulfur, subbituminous coal produced in the West will result in the continuation of the phenomenon of the last few years, i.e., more tonnage from fewer mines and more emphasis on the larger surface mines. Production in 1990 is expected to top 1 billion short tons.

Increasing use of longwall techniques in underground mines contributed to productivity increase. Twentyfive to 30 percent of the total underground production utilized longwall techniques. The primary mining method was still the use of continuous mining machines which accounted for over half of the underground production.

Wyoming led the Nation in production in 1989. Production in Wyoming rose 5 percent to almost 172 million short tons, 170 million of which came from surface 
mines. Wyoming's Campbell County, the Nation's top producing county, mined about 144 million short tons. The second.largest producing State, Kentucky, mined about 166 million short tons, followed by West Virginia with a little over 153 million.

\section{Figure 1. Coal Production by Region, 1989}

(Million Short Tons)

$$
\begin{aligned}
\text { Total }=977.5 & \\
& \text { Appalachian } \\
& 461.5 \\
& 47.2 \%
\end{aligned}
$$

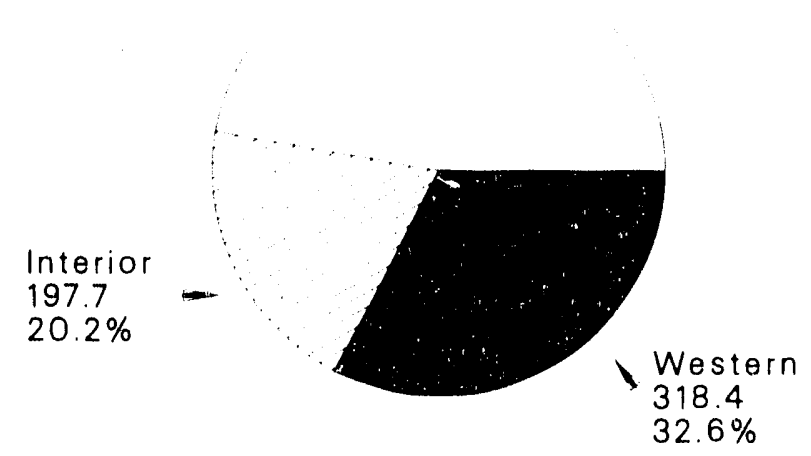

Source: Energy Information Administration, Form EIA-7A, "Coal Production Report."

\section{Number of Mines}

The number of active coal mines in the United States $f_{2}$ ' 3 percent, to 2,821 mines (1,426 underground and 1,395 surface mines). Most of these mines are located in the Appalachian Region, which has almost 90 percent of all mines nationally. These mines accounted for almost half of U.S. production. Conversely, a relatively small number (64) of surface mines in the Western Region account for nearly 30 percent of total production. The Appalachian Region lost 79 mines, for a total of 2,484 . The number of surface mines in the Interior and Western Regions fell, but the number of underground operations rose.

\section{Mines}

In 1989, there were 209 mines that produced one million or more short tons. Although these mines represented only 7 percent of the total active mines, they accounted for almost two-thirds of the total national production. As in recent years, Thunder Basin Coal Company's Black Thunder surface mine in Wyoming produced the most coal of any single mine, close to 30 million short tons. Consolidation Coal Company's Bailey No. 1 mine in Pennsylvania continued as the Nation's top producing underground mine with 6 million tons.

\section{Coal Rank}

More than two-thirds (657 million short tons) of the coal mined in the United States in 1989 was bituminous coal. Most bituminous coal is produced in the Appalachian Region; Kentucky led in state bituminous coal production with more than 166 million short tons. Subbituminous coal accounted for 24 percent of production, about 231 million short tons. Wyoming produced the most subbituminous coal in 1989, more than 169 million short tons. Texas was the leading producer of lignite, mining close to 54 million short tons out of a national yield of 86 million short tons. Pennsyivania anthracite production declined 7 percent in 1989 to under 3 million short tons, less than 1 percent of national coal production.

\section{Major Coalbeds}

A major coalbed is one from which at least 10 million short tons of coal are produced in a year. The 19 major coalbeds in 1989 yielded more than 578 million short tons, about 59 percent of national production. The three leading coalbeds, as in 1988, were the Wyodak in W.uming (over 143 million short tons), the Pittsburgh in the northern Appalachian Region (60 million short tons), and the No. 6 in Illinois (50 million short tons), for a combined total of over 253 million short tons.

\section{Average Mine Price}

The average mine price is determined by taking the ratio of the f.o.b. value of coal produced at the mine to the total production tonnage. The average mine price of U.S. coal fell for the seventh straight year, to 
$\$ 21.82$ per short ton. This was the first time since the late seventies that an average ton of coal sold for less than $\$ 22.00$. Factors contributing to the falling price were the growing demand for low cost, low sulfur, subbituminous coal and lignite, improved mining technology, and increased productivity. Subbituminous coal and lignite are cheaper to produce, and are abundant West of the Mississippi, where electric utility coal demand has been on the rise. Subbituminous coal and lignite inade up about one-third of the output in 1989 , and cost $\$ 10.16$ and $\$ 9.91$ per short ton, respectively, as compared to $\$ 27.40$ per short ton for bituminous coal. The price of surface-mined coal fell 5 cents in 1989 , to $\$ 17.38$ per short ton, while the price of underground coal dropped 53 cents, to $\$ 28.44$ per short ton.

\section{Productivity}

Productivity is measured by the amount of coal produced by a nuiner in an hour. At 3.7 tons per miner per hour, productivity in 1989 set a record for the third year in a row, an increase of over 4 percent. The primary reason for this was the surge in Western surface mining, where conditions favor the more efficient mechanized techniques. Productivity in Western surface mining climbed to nearly 15 short tons an hour. In the East increased use of techniques such as longwall mining and continuous mining contributed to the improved efficiency in underground production.

\section{Employment}

The average number of miners working daily fell 3 percent in 1989, to about 131,000. Recent trends had declines of about 5 percent in the number of miners annually. The greatest losses were in the Interior Region, with over 2,200 mining jobs lost. Among Interior surface miners, the change has been the most dramatic: from 13,000 surface miners in 1988 to just over 10,000 in 1989, a 21 percent drop. In the top producing States, the number of jobs rose 6 percent in Wyoming, remained about the same in Kentucky, and rose 2 percent in West Virginia.

\section{Reserves}

The two principal types of coal reserves are the Demonstrated Reserve Base (DRB) and recoverable reserves. DRB is an estimate of all potentially minable coal regardless of the legal, technical or economic restrictions. (DRB is more fully explained in Appendix A.) Recover'able reserves are the amount of coal that can be produced at an active site. About 15.3 billion short tons are recoverable by current methods of surface mining, and 7.4 billion short tons are recoverable by existing underground methods. Recoverable reserves fell about 4 percent in 1989. Wyoming has the greatest amount in recoverable reserves, about 6.3 billion short tons, followed by West Virginia with 2.3 billion short tons, and Kentucky and Montana, with about 1.5 billion short tons each. 


\section{Number of Mines and Production}

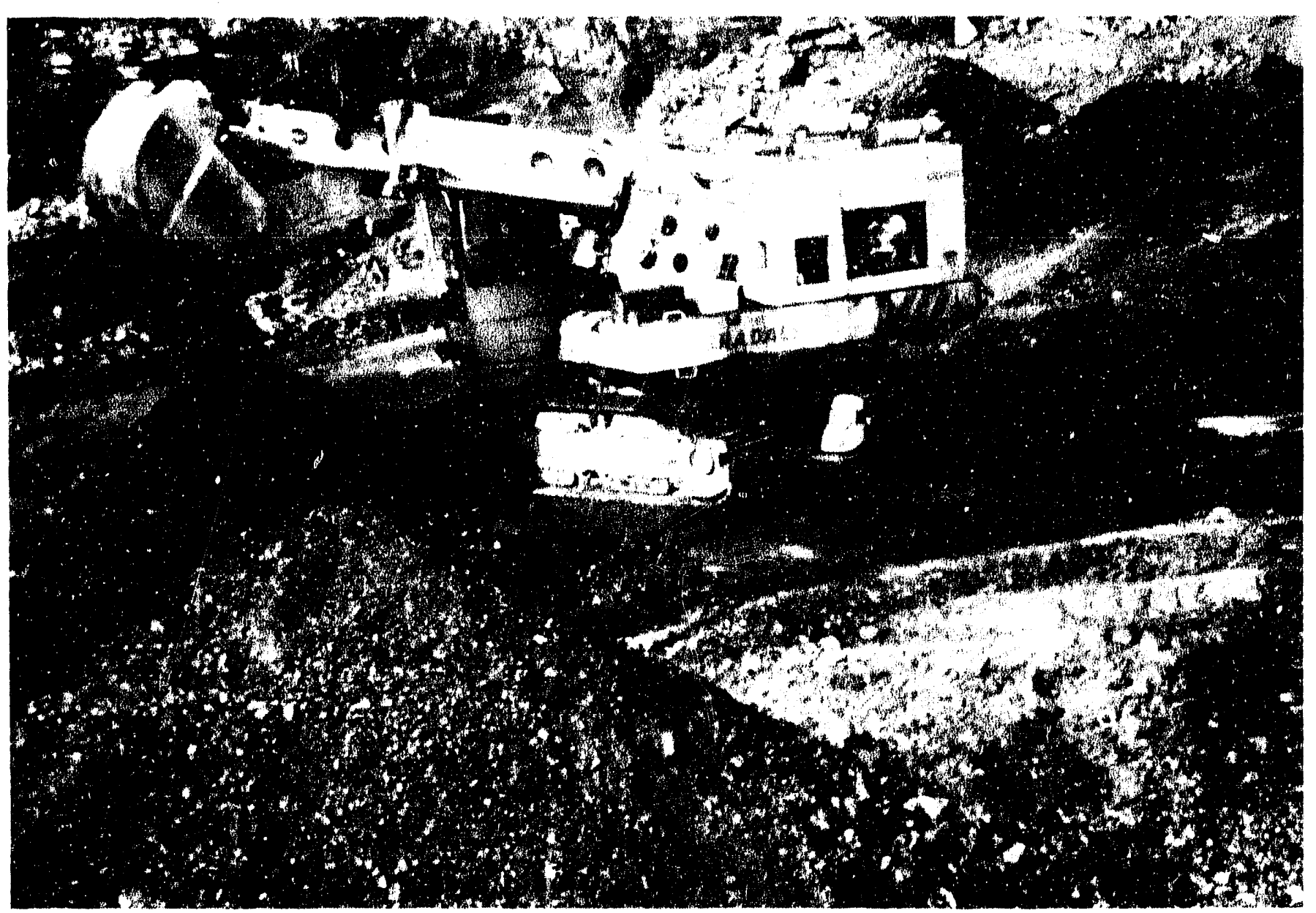

Hydraulic excavators can selectively axavate, mine, and handle coal at surface mines. 
Figure 2. U.S. Coal Production and Number of Mines by Type of Mining and by Region, 1980-1989
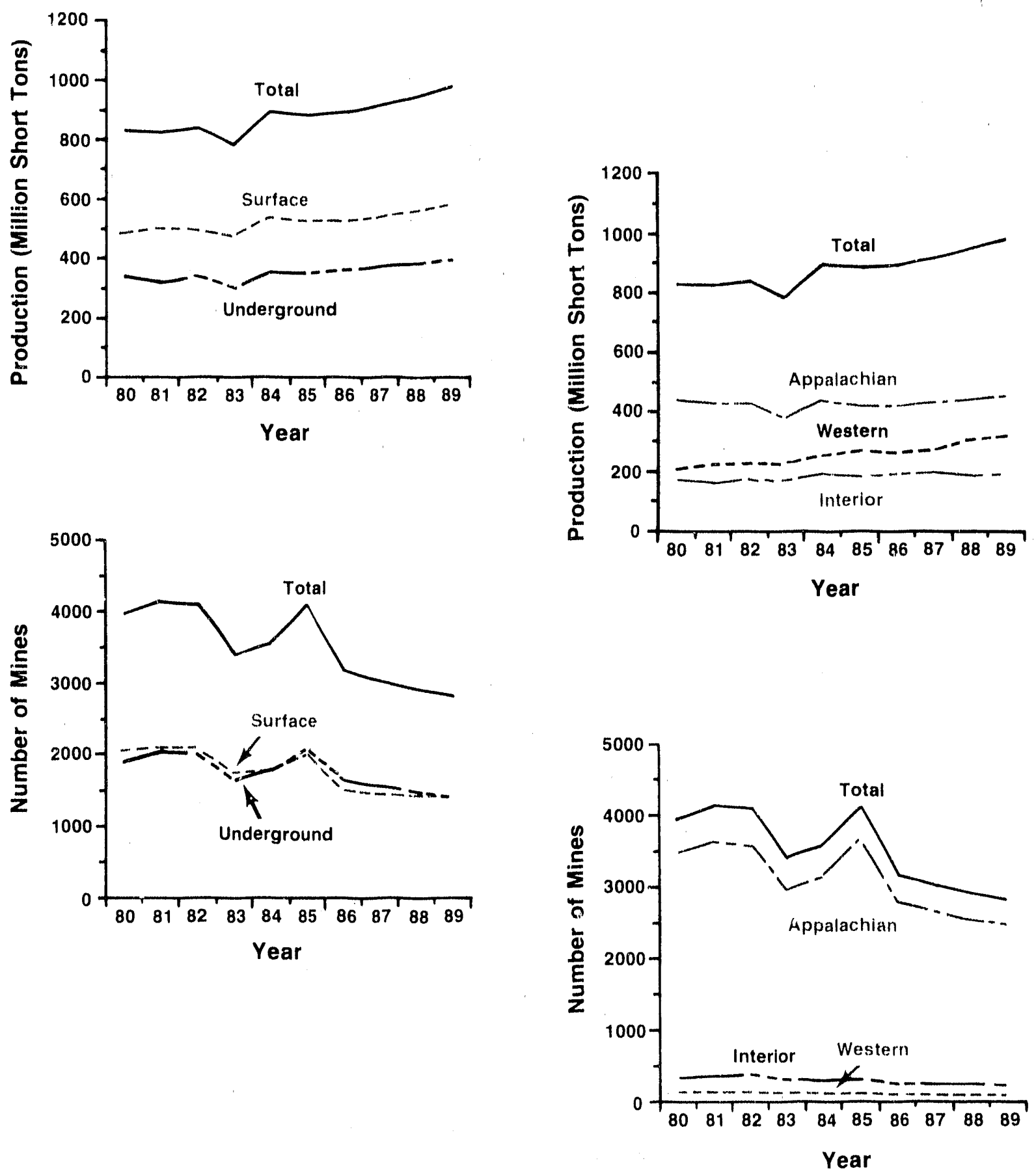

Source: Energy Information Administration, Form ElA-7A, "Coal Production Report." 
Table 2. U.S. Coal Production ${ }^{1}$ and Number of Mines by State and Type of Mining, 1989

(Thousand Short Tons)

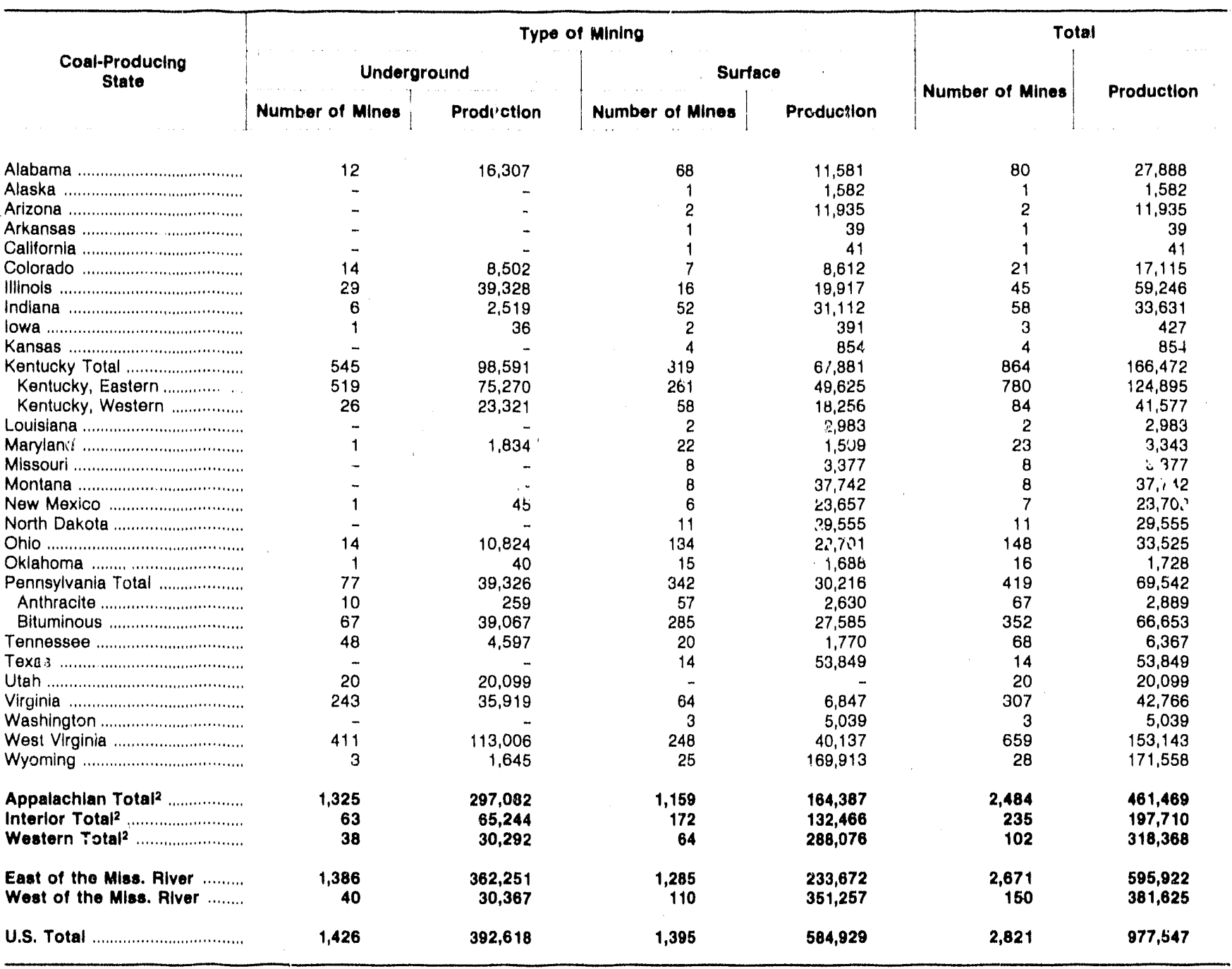

1 Excludes silt, culm, refuse bank, slurry dam, and dredge production except for Pennsylvania anthracite. Excludes mines producing less than 10,000 short tons of coal during the year.

2 For a definition of coal-producing regions, see Appendix C.

Note: Total may not equal sum of components because of independent rounding.

Source: Energy Information Administration, Form ElA-7A, "Coal Production Report." 
Figure 3. U.S. Coal Production by State, 1989

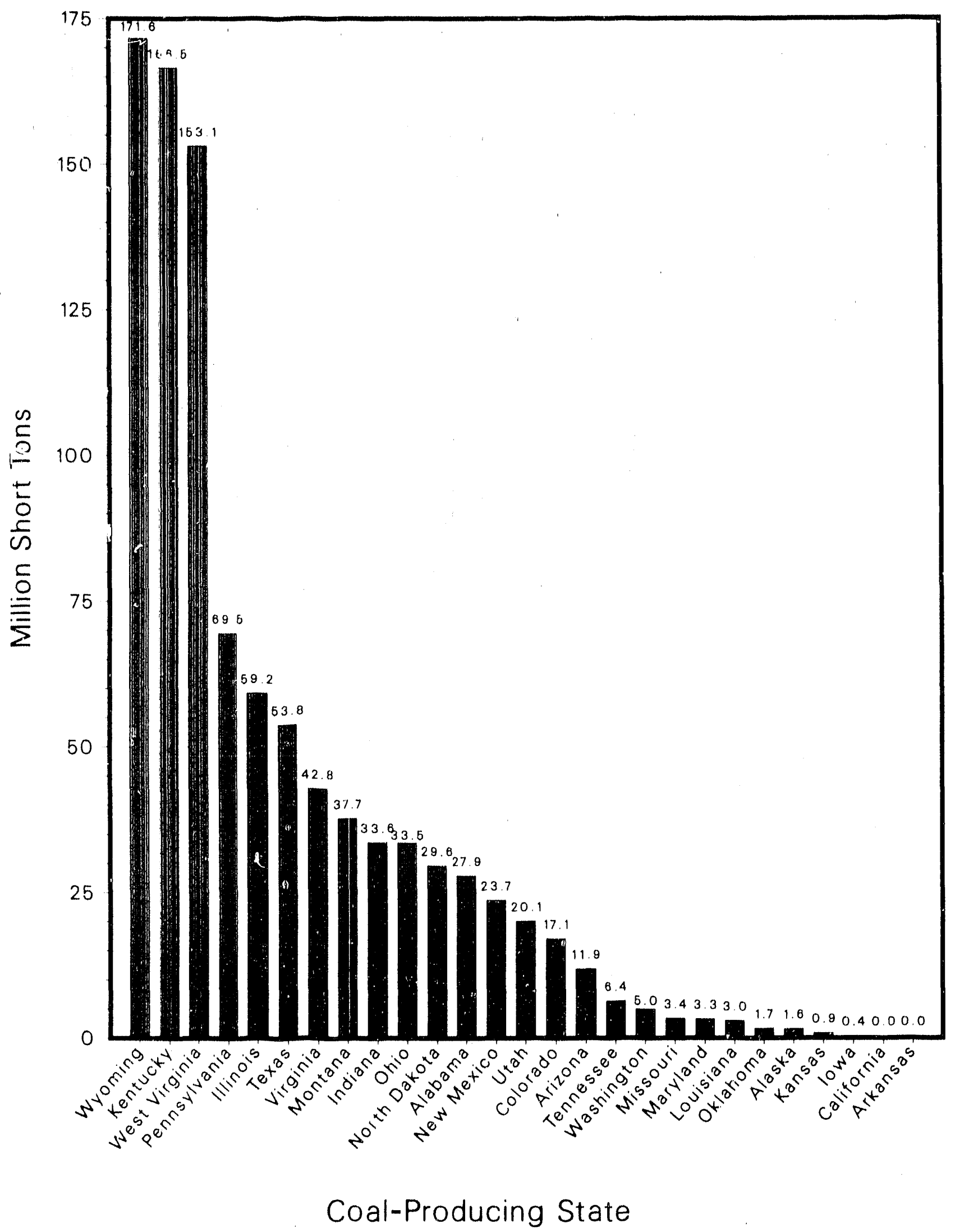

Note: Production for Arkansas and California was less than 0.1 million short tons

Source: Energy information Administration. Form ElA-7A, "Coal Production Report." 
Table 3. U.S. Coal Production ${ }^{1}$ and Number of Mines by District and Type of Mining, 1989

(Thousand Short Tons)

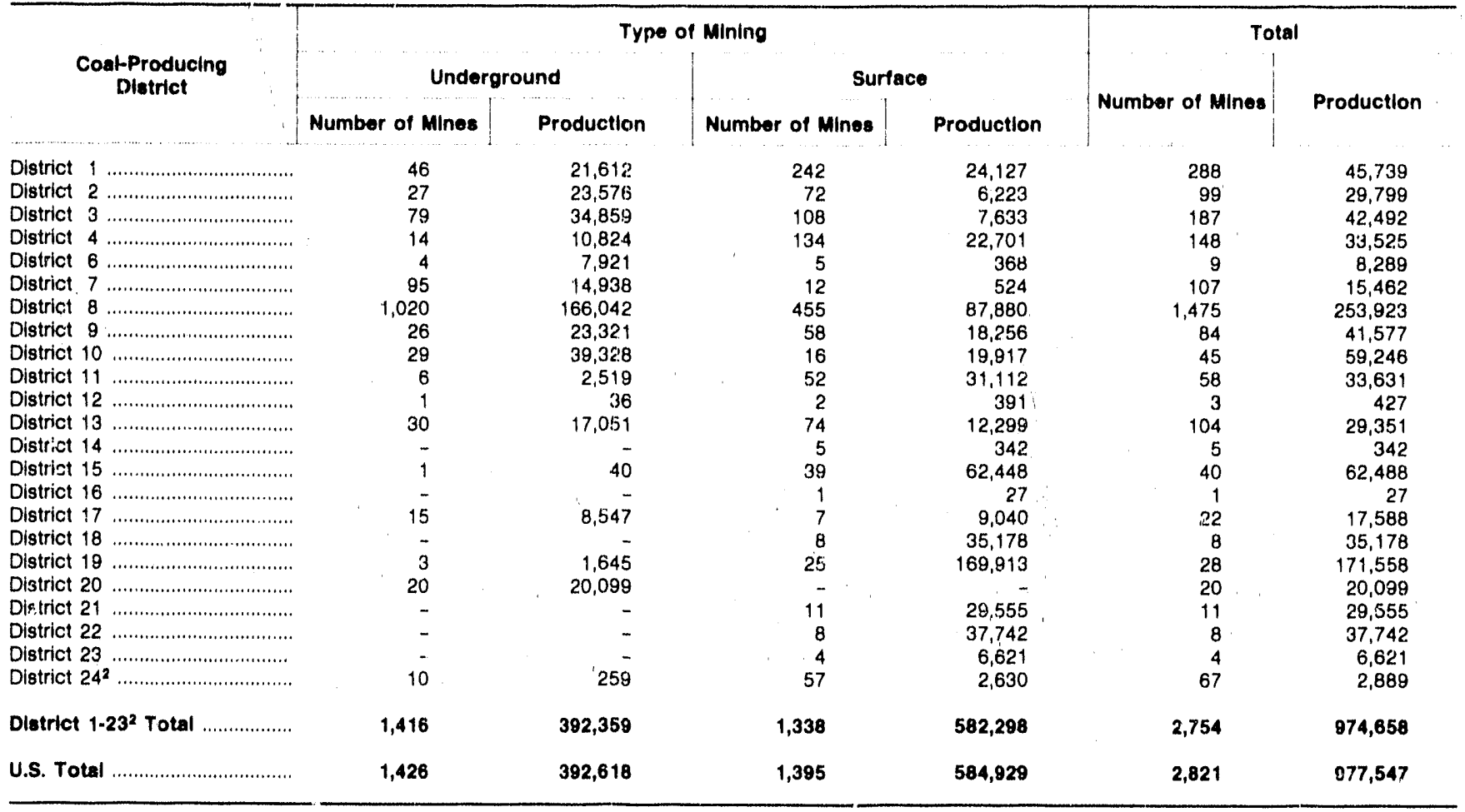

1 Excludes silt, culm, refuse bank, slurry dam, and dredge production except for Pennsylvania anthracite. Excludes mines producing less than 10,000 short tons of coal during the year.

2 District 24 is the anthracite-producing district in Pennsylvania. Districts 1-23 represent the total U.S. production of bituminous, subbituminous coal, and lignite. See Appendix C.

Note: Total may not equal sum of components because of independent rounding.

Source: Energy Information Administration, Form ElA-7A, "Coal Production Repoit." 
Table 4. U.S. Coal Production ${ }^{1}$ and Number of Mines by State and County and Type of Mining, 1989

(Thousand Short Tons)

\begin{tabular}{|c|c|c|c|c|c|c|}
\hline \multirow{3}{*}{$\begin{array}{l}\text { Coal-Proc ucing } \\
\text { State and County }\end{array}$} & \multicolumn{4}{|c|}{ Type of Mining } & \multicolumn{2}{|c|}{ Total } \\
\hline & \multicolumn{2}{|c|}{ Underground } & \multicolumn{2}{|c|}{ Surface } & \multirow[b]{2}{*}{ Number of Mines } & \multirow[b]{2}{*}{ Production } \\
\hline & Number of Mines & Production & Number of Mines & Production & & \\
\hline Alabama & 12 & 16,307 & 68 & 11,581 & 80 & 27,888 \\
\hline 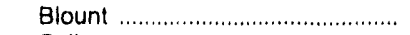 & - & - & 5 & 202 & 5 & 202 \\
\hline 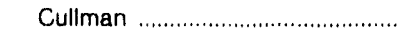 & - & - & 1 & 453 & 1 & 453 \\
\hline 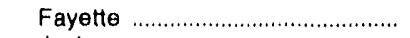 & 1 & 2,022 & 1 & 14 & 2 & 2,036 \\
\hline 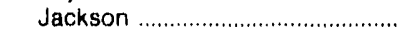 & - & & 1 & 28 & 1 & 28 \\
\hline 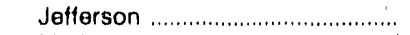 & 5 & 4,500 & 9 & 2,272 & 14 & 6,771 \\
\hline Marion & 1 & 20 & 6 & 292 & 7 & 311 \\
\hline 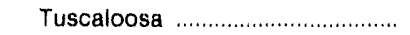 & 3 & 6,907 & 17 & 3,014 & 20 & 9,921 \\
\hline 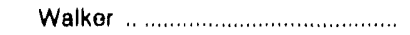 & 2 & 2,857 & 23 & 4.721 & 25 & 7,578 \\
\hline Winston & - & - & 5 & 587 & 5 & 587 \\
\hline Alaska .............................................. & - & - & 1 & 1,582 & 1 & 1,582 \\
\hline 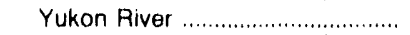 & - & - & 1 & 1,582 & 1 & 1,582 \\
\hline Arlzona & - & - & 2 & 11,935 & 2 & 11,935 \\
\hline 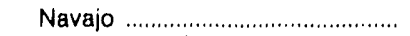 & - & - & 2 & 11,935 & 2 & 11,935 \\
\hline Arkansas & - & - & 1 & 39 & 1 & 39 \\
\hline 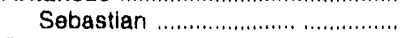 & .. & - & $i$ & 39 & 1 & 39 \\
\hline Callfornla ........................................ & - & - & 1 & 41 & 1 & 41 \\
\hline Amador & - & - & 1 & 41 & 1 & 41 \\
\hline Colorado & 14 & 8,502 & 7 & 8,612 & 21 & 17,115 \\
\hline 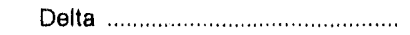 & 1 & 517 & - & - & 1 & 517 \\
\hline 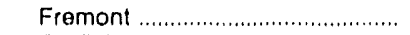 & 2 & 499 & - & -. & 2 & 499 \\
\hline 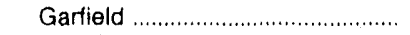 & 1 & 125 & - & - & 1 & 125 \\
\hline Gunnison ……………………............. & 2 & 860 & - & - & 2 & 860 \\
\hline Jackson & - & - & 1 & 27 & 1 & 27 \\
\hline La Plata ............................................ & 1 & 160 & .. & - & 1 & 160 \\
\hline 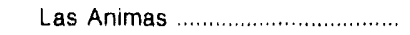 & 2 & 855 & - & - & 2 & 855 \\
\hline Mesa & 1 & 134 & - & - & 1 & 134 \\
\hline Moffat ……………………........ & 1 & 2,201 & 2 & 6,496 & 3 & 8,696 \\
\hline 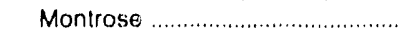 & - & - & 1 & 47 & 1 & 47 \\
\hline Pitkin & 1 & 935 & - & - & 1 & 935 \\
\hline 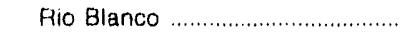 & 1 & 1,365 & - & - & 1 & 1,365 \\
\hline 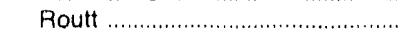 & 1 & 851 & 3 & 2,043 & 4 & 2,895 \\
\hline 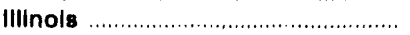 & 29 & 39,328 & 16 & 19,917 & 45 & 59,246 \\
\hline 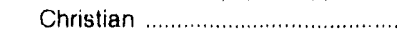 & 1 & 1,876 & - & - & 1 & 1,876 \\
\hline Clinton & 1 & 2,762 & - & - & 1 & 2,762 \\
\hline Coles & 1 & 12 & - & - & 1 & 12 \\
\hline 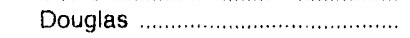 & 1 & 1,051 & - & .. & 1 & 1,051 \\
\hline 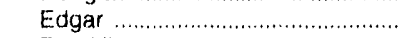 & - & & 1 & 233 & 1 & 233 \\
\hline Franklin & 4 & 7,540 & - & - & 4 & 7,540 \\
\hline 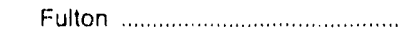 & - & - & 1 & 504 & 1 & 504 \\
\hline Gallatin & 2 & 1,702 & 1 & 531 & 3 & 2,233 \\
\hline Jackson & - & & 1 & 2,160 & 1 & 2,160 \\
\hline Jefferson …………………....... & 2 & 3,573 & - & - & 2 & 3,573 \\
\hline Logan & 1 & 1,329 & - & - & 1 & 1,329 \\
\hline 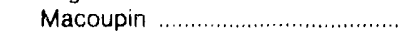 & 2 & 2,809 & - & - & 2 & 2,809 \\
\hline McDonough & - & - & 1 & 516 & 1 & 516 \\
\hline 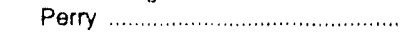 & 1 & 204 & 6 & 11,114 & 7 & 11,318 \\
\hline 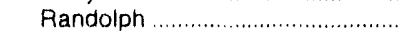 & 3 & 4,725 & 1 & 1,404 & 4 & 6,129 \\
\hline Saline & 4 & 4,877 & 3 & 2,895 & 7 & 7,772 \\
\hline Sangamon & 1 & 173 & - & - & 1 & 173 \\
\hline 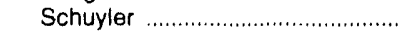 & - & - & 1 & 560 & 1 & 560 \\
\hline St Clair & 2 & 1,295 & - & - & 2 & 1,295 \\
\hline 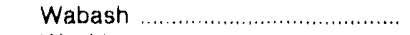 & 1 & 1,787 & - & - & 1 & 1,787 \\
\hline 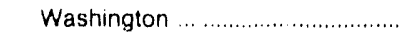 & 1 & 1,862 & - & - & 1 & 1,862 \\
\hline White & 1 & 1,751 & - & - & 1 & 1,751 \\
\hline Indlana & 6 & 2,519 & 52 & 31,112 & 58 & 33,631 \\
\hline 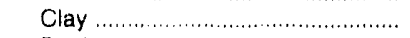 & - & - & $B$ & 2,112 & 8 & 2,112 \\
\hline 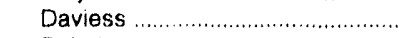 & - & .. & 11 & 4,671 & 11 & 4,671 \\
\hline 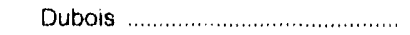 & - & - & 2 & 581 & 2 & 581 \\
\hline 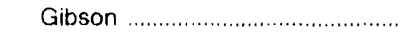 & 1 & 1,215 & 2 & 1,237 & 3 & 2,452 \\
\hline Greene & 2 & 591 & 5 & 2,021 & 7 & 2,612 \\
\hline Knox & 1 & 366 & 1 & 255 & 2 & 622 \\
\hline Pike & 1 & 72 & 6 & 5,209 & 7 & 5,282 \\
\hline Spencer & - & - & 3 & 535 & 3 & $53 E$ \\
\hline Sullivan & 1 & 275 & 2 & 2,653 & 3 & 2,827 \\
\hline Vermillion & - & - & 1 & 1,447 & 1 & 1,447 \\
\hline Vigo & - & - & 4 & 1,415 & 4 & 1,415 \\
\hline 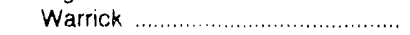 & - & - & 7 & 8,976 & 7 & 8,976 \\
\hline
\end{tabular}

See footnotes at end of table. 
Table 4. U.S. Coal Production ${ }^{1}$ and Number of Mines by State and County and Type of Mining, 1989 (Coritinued) (Thousand Short Tons)

\begin{tabular}{|c|c|c|c|c|c|c|}
\hline \multirow{3}{*}{$\begin{array}{l}\text { Coal-Producing } \\
\text { State anid County }\end{array}$} & \multicolumn{4}{|c|}{ Type of Mining } & \multicolumn{2}{|c|}{ Totell } \\
\hline & \multicolumn{2}{|c|}{ Underground } & \multicolumn{2}{|c|}{ Surface } & \multirow{2}{*}{ Number of MInes } & \multirow{2}{*}{ Productlon } \\
\hline & Number of Mines & Production & Number of Minb & Production & & \\
\hline 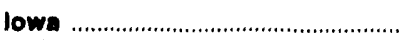 & 1 & 36 & 2 & 391 & 3 & 427 \\
\hline 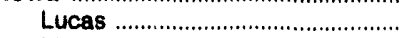 & $i$ & 36 & - & - & 1 & 36 \\
\hline Marion & - & - & 2 & 394 & 2 & 391 \\
\hline 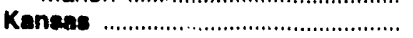 & - & - & 4 & 854 & 4 & 854 \\
\hline 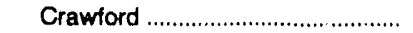 & - & - & 3 & 624 & 3 & 624 \\
\hline 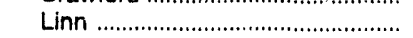 & - & - & 1 & 230 & 1 & 230 \\
\hline 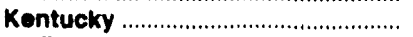 & 545 & 98,591 & 319 & 67,881 & 864 & 166,472 \\
\hline 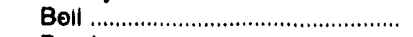 & 22 & 2,488 & 15 & 2,256 & 37 & 4,744 \\
\hline 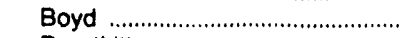 & - & - & 1 & 603 & 1 & 603 \\
\hline 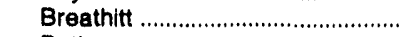 & - & - & 10 & 6,199 & 10 & 6,199 \\
\hline 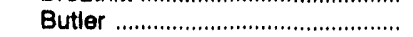 & - & - & 5 & 937 & 5 & 937 \\
\hline 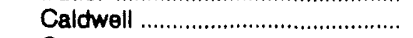 & - & - & 1 & 48 & 1 & 48 \\
\hline 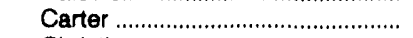 & - & - & 2 & 109 & 2 & 109 \\
\hline 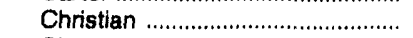 & - & - & 2 & 540 & 2 & 540 \\
\hline 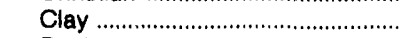 & 1 & 261 & 5 & 441 & 6 & 702 \\
\hline 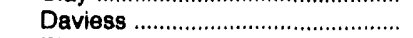 & - & - & 7 & 1,529 & 7 & 1,529 \\
\hline 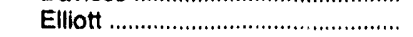 & - & - & 2 & 37 & 2 & 37 \\
\hline Floyd & 63 & 5,627 & 14 & 4,140 & 77 & 9,767 \\
\hline Greenup & - & - & 1 & 90 & 1 & 90 \\
\hline Harlan & 97 & 12,650 & 20 & 1,513 & 117 & 14,162 \\
\hline 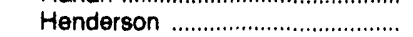 & 2 & 1,647 & 2 & 1,356 & 4 & 3,003 \\
\hline 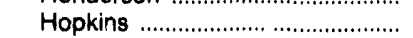 & 6 & 3,398 & 18 & 6,909 & 24 & 10,306 \\
\hline 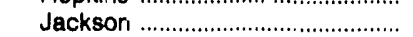 & - & - & 3 & 265 & 3 & 265 \\
\hline 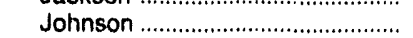 & 10 & 1,772 & 2 & 76 & 12 & 1,848 \\
\hline Knott & 33 & 4,893 & 22 & 3,739 & 55 & 8,633 \\
\hline Knox & 23 & 483 & 9 & 567 & 32 & 1,050 \\
\hline 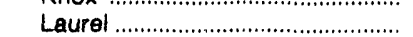 & - & 400 & 3 & 47 & 3 & 47 \\
\hline Lawrence & 1 & 15 & 4 & 164 & 5 & 178 \\
\hline Le & - & - & 5 & 167 & 5 & 167 \\
\hline 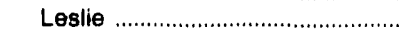 & 15 & 6,654 & 5 & 1,663 & 20 & 8,317 \\
\hline 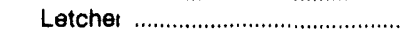 & 34 & 6,386 & 26 & 3,715 & 60 & 10,100 \\
\hline 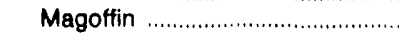 & 5 & 375 & 12 & 1,884 & 17 & 2,259 \\
\hline Martin & 20 & 7,854 & 10 & 4,917 & 30 & 12,772 \\
\hline MoLeen & - & 1,00 & 3 & 468 & 3 & 468 \\
\hline 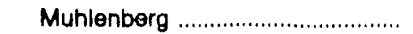 & 5 & 3,847 & 8 & 2,675 & 13 & 6,522 \\
\hline Ohio & 1 & 114 & 9 & 1,539 & 10 & 1,653 \\
\hline 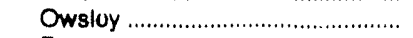 & - & - & 6 & 175 & 6 & 175 \\
\hline Porry & 14 & 2,565 & 22 & 8,608 & 36 & 11,173 \\
\hline 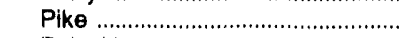 & 167 & 22,442 & 4.5 & 6,762 & 212 & 29,204 \\
\hline 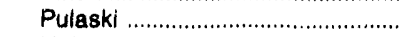 & - & - & 2. & 313 & 2 & 313 \\
\hline Union & 5 & 6,396 & 1 & 118 & 6 & 6,513 \\
\hline 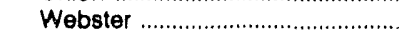 & 7 & 7,810 & 2 & 2,138 & 9 & 10,057 \\
\hline 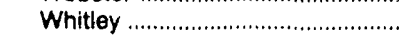 & 14 & 806 & 13 & 1,142 & 27 & 1,948 \\
\hline Wolfo & - & - & 2 & 33 & 2 & 33 \\
\hline 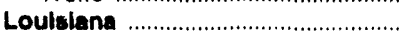 & - & - & 2 & 2,883 & 2 & 2,983 \\
\hline 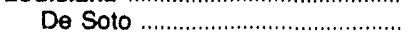 & - & - & $i$ & 2,880 & 1 & 2,880 \\
\hline Red River & - & - & 1 & 103 & 1 & 103 \\
\hline 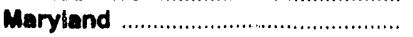 & 9 & 1,834 & 22 & 1,509 & 23 & 3,343 \\
\hline Allegeny & - & - & 13 & 836 & 13 & 836 \\
\hline Garrett & 1 & 1,834 & 9 & 673 & 10 & 2,506 \\
\hline 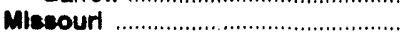 & .. & - & 8 & 3,377 & 8 & 3,377 \\
\hline Barton & - & - & 1 & 527 & 1 & 527 \\
\hline Bates & - & - & 1 & 186 & 1 & 186 \\
\hline 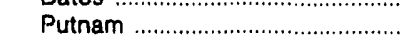 & - & - & 1 & 41 & 1 & 41 \\
\hline Ralls & - & - & 1 & 76 & 1 & 76 \\
\hline 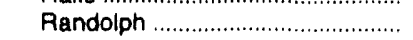 & - & - & 2 & 2,399 & 2 & 2,399 \\
\hline Vernon & - & - & 2 & 148 & 2 & 148 \\
\hline 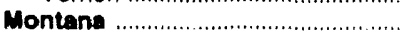 & - & - & 8 & 37,742 & 8 & 37,742 \\
\hline 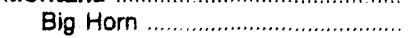 & - & - & 4 & 20,044 & 4 & 20,044 \\
\hline 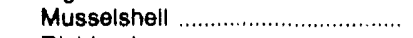 & - & - & 1 & 16 & 1 & 16 \\
\hline 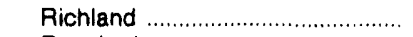 & - & - & 1 & 288 & 1 & 288 \\
\hline 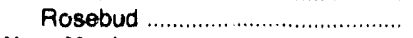 & - & - & 2 & 17,395 & 2 & 17,385 \\
\hline 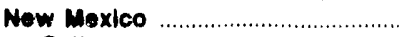 & 1 & 45 & 6 & 23,657 & 7 & 23,702 \\
\hline Colfax & $i$ & 45 & 1 & 455 & 2 & 500 \\
\hline 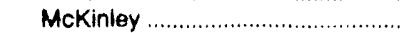 & - & - & 2 & $8,18.3$ & 2 & 8,183 \\
\hline 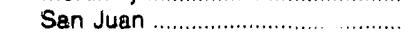 & - & - & 3 & 15,019 & 3 & 15,019 \\
\hline
\end{tabular}

Seo Footnotes at end of table 
Table 4. U.S. Coal Production ${ }^{1}$ and Number of Mines by State and County and Type of Mining, 1989 (Continued) (Thousand Short Tons)

\begin{tabular}{|c|c|c|c|c|c|c|}
\hline \multirow{3}{*}{$\begin{array}{l}\text { Soai-Producing } \\
\text { State and County }\end{array}$} & \multicolumn{4}{|c|}{ Type of Mining } & \multicolumn{2}{|c|}{ Total } \\
\hline & \multicolumn{2}{|c|}{ Underground } & \multicolumn{2}{|c|}{ Surface } & \multirow{2}{*}{ Number of Mines } & \multirow{2}{*}{ Production } \\
\hline & Number of Mines & Production & Number of Mines & Production & & \\
\hline 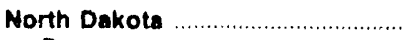 & - & - & 11 & 29,555 & 11 & 29,555 \\
\hline 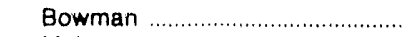 & - & - & 1 & 2,015 & 1 & 2,015 \\
\hline 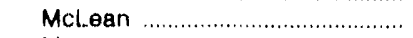 & - & - & 1 & 6,725 & 1 & 6,725 \\
\hline 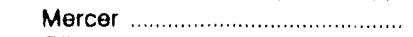 & - & - & 5 & 14,757 & 5 & 14,757 \\
\hline 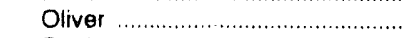 & - & - & 3 & 5,862 & 3 & 5,862 \\
\hline 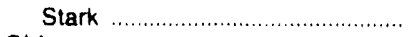 & - & - & 1 & 195 & 1 & 195 \\
\hline 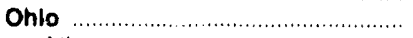 & 14 & 10,824 & 134 & 22,701 & $14 \mathrm{e}$ & 33,525 \\
\hline Athens & - & - & 1 & 151 & 1 & 151 \\
\hline 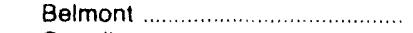 & 2 & 2,098 & 12 & 2,614 & 14 & 4,712 \\
\hline Carroll ......................................... & - & - & 7 & 874 & 7 & 874 \\
\hline Columbiana ............................... & 4 & 529 & 14 & 778 & 18 & 1,307 \\
\hline 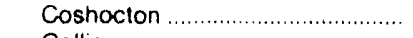 & - & - & 8 & 2,118 & 8 & 2,118 \\
\hline 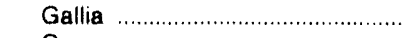 & - & - & 1 & 23 & 1 & 23 \\
\hline 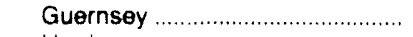 & - & - & 3 & 138 & 3 & 138 \\
\hline 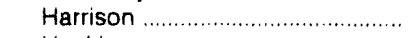 & 1 & 516 & 8 & 2,216 & 9 & 2,732 \\
\hline 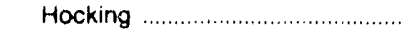 & - & - & 6 & 238 & 6 & 238 \\
\hline 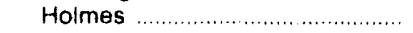 & - & - & 5 & 593 & 5 & 593 \\
\hline 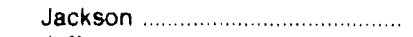 & 1 & 48 & 7 & 1,232 & 8 & 1,280 \\
\hline 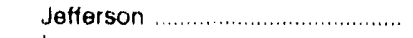 & - & - & 14 & 1,818 & 14 & 1,818 \\
\hline 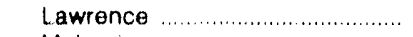 & - & - & 2 & 642 & 2 & 642 \\
\hline 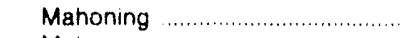 & - & - & $i$ & 193 & 1 & 193 \\
\hline 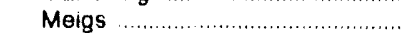 & 2 & 3,942 & - & - & 2 & 3,842 \\
\hline Monroe & 1 & 1,981 & - & - & 1 & 1,981 \\
\hline 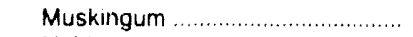 & 1 & 33 & 9 & 1,189 & 10 & 1,222 \\
\hline Noble & - & - & 5 & 3,361 & 5 & 3,361 \\
\hline 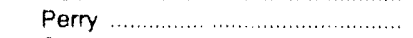 & 1 & 1.161 & 3 & 408 & 4 & 1,568 \\
\hline 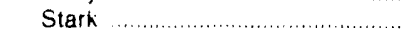 & - & - & 3 & 164 & 3 & 164 \\
\hline 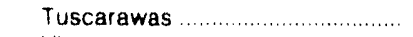 & - & - & 18 & 2,230 & 18 & 2,230 \\
\hline 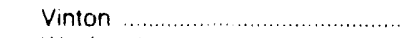 & 1 & 517 & 6 & 1,675 & 7 & 2,192 \\
\hline 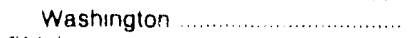 & - & - & 1 & 47 & 1 & 47 \\
\hline Oklahoma & 1 & 40 & 15 & 1,688 & 16 & 1,728 \\
\hline 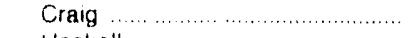 & - & - & 6 & 662 & 6 & 662 \\
\hline 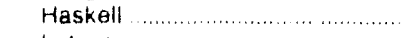 & - & - & 2 & 207 & 2 & 207 \\
\hline Latımer .................................... & . & - & 1 & 243 & 1 & 243 \\
\hline 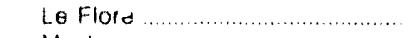 & - & - & 2 & 96 & 2 & 96 \\
\hline 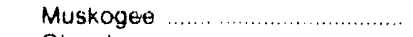 & - & - & 2 & 138 & 2 & 138 \\
\hline 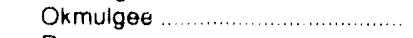 & 1 & 40 & - & - & 1 & 40 \\
\hline 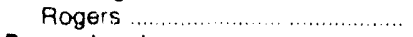 & $*$ & - & 2 & 343 & 2 & 343 \\
\hline 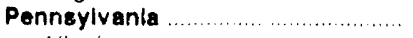 & 77 & 39,326 & 342 & 30,216 & 418 & 69,542 \\
\hline 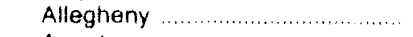 & 3 & 822 & 3 & 331 & 6 & 1,152 \\
\hline 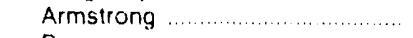 & 11 & 3,621 & 28 & 1,516 & 39 & 5,137 \\
\hline 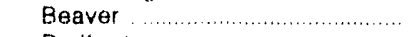 & - & - & 2 & 377 & 2 & 377 \\
\hline 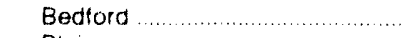 & - & - & 1 & 24 & 1 & 24 \\
\hline 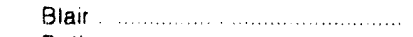 & - & - & 1 & 143 & 1 & 143 \\
\hline 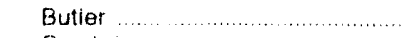 & 4 & 959 & 14 & 1,052 & 18 & 2,011 \\
\hline 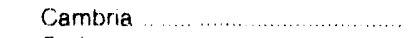 & 4 & 2,153 & 13 & 2,401 & 17 & 4,554 \\
\hline 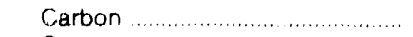 & - & - & 1 & 57 & 1 & 57 \\
\hline 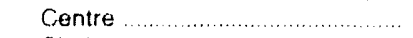 & 1 & 687 & 6 & 386 & 7 & 1,073 \\
\hline 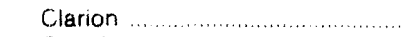 & - & - & 12 & 2,729 & 12 & 2,729 \\
\hline 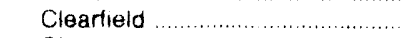 & 1 & 79 & 57 & 5,761 & 58 & 5,840 \\
\hline 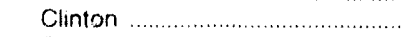 & - & - & 3 & 202 & 3 & 202 \\
\hline Columbia & - & - & 2 & 64 & 2 & 64 \\
\hline 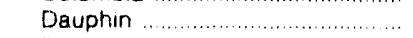 & - & - & 1 & 17 & 1 & 17 \\
\hline Elk & - & - & 7 & 335 & 7 & 335 \\
\hline Fayette & - & - & 16 & 573 & 16 & 573 \\
\hline 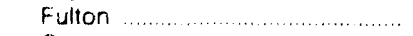 & - & - & 1 & 93 & 1 & 93 \\
\hline 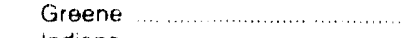 & 14 & 15,$3 ; 1$ & 6 & 321 & 20 & 15,632 \\
\hline Indiana & 16 & 7,860 & 20 & 1,390 & 36 & 9,250 \\
\hline 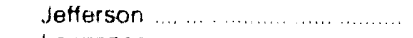 & 3 & 673 & 29 & 2,107 & 32 & 2.780 \\
\hline 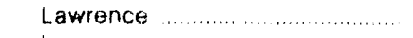 & - & - & 8 & 1,080 & 8 & 1,080 \\
\hline 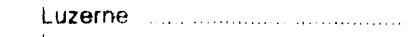 & - & - & 15 & 613 & 15 & 613 \\
\hline 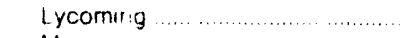 & - & - & 1 & 254 & 1 & 254 \\
\hline 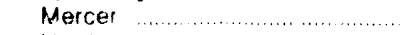 & $\cdots$ & - & 3 & 359 & 3 & 359 \\
\hline Northumberland ..................... & 2 & 23 & 3 & 71 & 5 & 84 \\
\hline 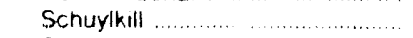 & 8 & 236 & 34 & 1,769 & 42 & 2,005 \\
\hline 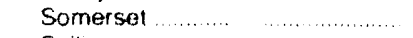 & 7 & 1,641 & 33 & 4.203 & 40 & 5,844 \\
\hline 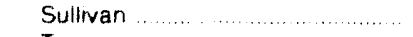 & - & - & 1 & 39 & 1 & 39 \\
\hline 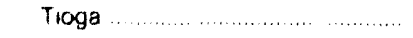 & - & - & 1 & 139 & 1 & 139 \\
\hline
\end{tabular}

See Footnotes at end of table. 
Table 4. U.S. Coal Production ${ }^{1}$ and Number of Mines by State and County and Type of Mining, iভ̄ê (Continued) (Thousand Short Tons)

\begin{tabular}{|c|c|c|c|c|c|c|}
\hline \multirow{4}{*}{$\begin{array}{l}\text { Coal-Producing } \\
\text { State and County }\end{array}$} & \multicolumn{4}{|c|}{ Type of MIning } & \multicolumn{2}{|c|}{ Total } \\
\hline & \multicolumn{2}{|c|}{ Underground } & \multicolumn{2}{|c|}{ Surface } & \multicolumn{2}{|l|}{ 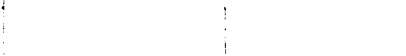 } \\
\hline & & & & & Number of Minos & Production \\
\hline & Number of Mines & Production & Number of Mines & Production & & \\
\hline \multicolumn{7}{|l|}{ Pennayivania (Continued) } \\
\hline 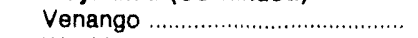 & - & - & 3 & 545 & 3 & 545 \\
\hline 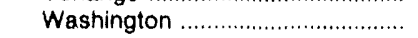 & 3 & 5,262 & 12 & 780 & 15 & 6,042 \\
\hline 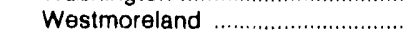 & - & - & 5 & 484 & 5 & 484 \\
\hline Tennesser & 48 & 4,507 & 20 & 1,770 & 68 & 6,367 \\
\hline 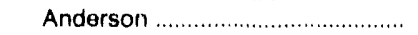 & 8 & 1,244 & 1 & 41 & 9 & 1,285 \\
\hline 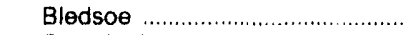 & 1 & 22 & 1 & 124 & 2 & 147 \\
\hline 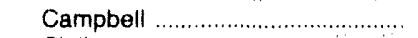 & 11 & 695 & 8 & 750 & 19 & 1,445 \\
\hline 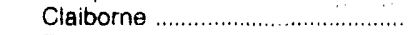 & 6 & 1.486 & 2 & 114 & 8 & 1,600 \\
\hline 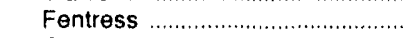 & - & - & 2 & 135 & 2 & 135 \\
\hline 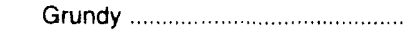 & - & - & 1 & 55 & 1 & 55 \\
\hline 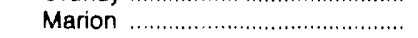 & 2 & 79 & - & - & 2 & 79 \\
\hline 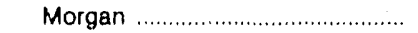 & 2 & 186 & - & - & 2 & 180 \\
\hline 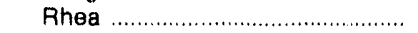 & 1 & 49 & - & - & 1 & 49 \\
\hline 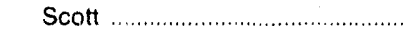 & 3 & 241 & 1 & 12 & 4 & 253 \\
\hline 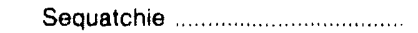 & 14 & 594 & 4 & 539 & 18 & 1,133 \\
\hline 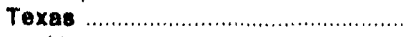 & - & - & 14 & 53,849 & 14 & 53,849 \\
\hline 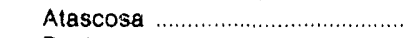 & - & - & 1 & 2,984 & 1 & 2,984 \\
\hline 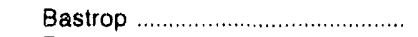 & - & - & 1 & 221 & 1 & 221 \\
\hline 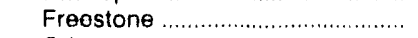 & - & - & 1 & 5,670 & 1 & 5,670 \\
\hline 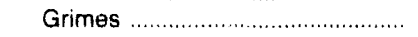 & - & - & 1 & ن 482 & 1 & 3,482 \\
\hline 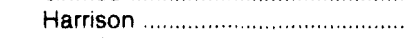 & - & - & 2 & 3,064 & 2 & 3,064 \\
\hline 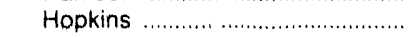 & - & - & 1 & 2,035 & 1 & 2,035 \\
\hline 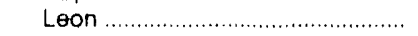 & - & - & 1 & 8,065 & 1 & 8,065 \\
\hline 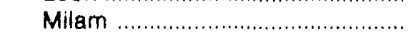 & - & - & 1 & 5,829 & 1 & 5,829 \\
\hline Panola & - & - & 1 & 8,554 & 1 & 8,554 \\
\hline Rusk & - & - & $i$ & 3,9213 & 1 & 3,928 \\
\hline 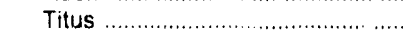 & - & - & 1 & 9,729 & 1 & 9,729 \\
\hline Webb & - & - & 2 & 287 & 2 & 287 \\
\hline Utah & $? 0$ & 20,099 & $=$ & - & 20 & 20,099 \\
\hline 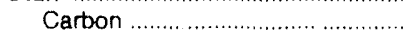 & 13 & 8,477 & - & - & 13 & 8,477 \\
\hline 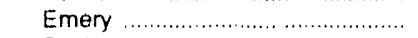 & 6 & 8.563 & - & - & 6 & 8.563 \\
\hline 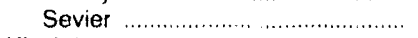 & 1 & 3,059 & -. & - & 1 & 3,059 \\
\hline 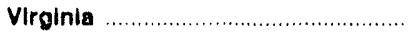 & 243 & 35,919 & 64 & 6,847 & 307 & 42,766 \\
\hline 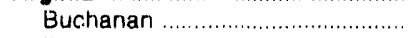 & 122 & 18,018 & 14 & 987 & 136 & 19,005 \\
\hline 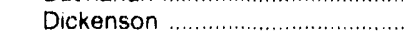 & 42 & 4,943 & 11 & 876 & 53 & 5,819 \\
\hline 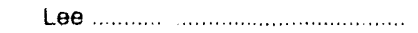 & 13 & 2,215 & 5 & 263 & 18 & 2,479 \\
\hline 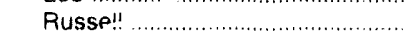 & 5 & 705 & 2 & 67 & 7 & 772 \\
\hline 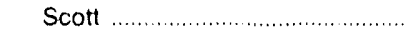 & 1 & 41 & - & - & 1 & 41 \\
\hline 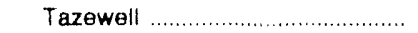 & 23 & 2,723 & .. & - & 23 & 2,723 \\
\hline 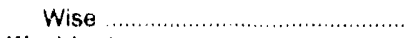 & 37 & 7,273 & 32 & 4,655 & 69 & 11,928 \\
\hline 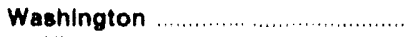 & - & - & 3 & 5,039 & 3 & 5,039 \\
\hline 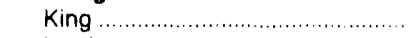 & - & - & 1 & 141 & 1 & 141 \\
\hline 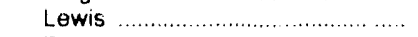 & - & - & 1 & 1,812 & 1 & 1,812 \\
\hline Thurston & - & - & 1 & 3,086 & 1 & 3,086 \\
\hline Weat Virginia & 411 & 113,006 & 248 & 40,137 & 659 & 153,143 \\
\hline Barbour & 7 & 1,585 & 9 & 687 & 16 & 2,272 \\
\hline 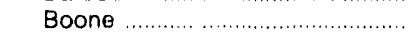 & 52 & 17,219 & 16 & 5,726 & 68 & 22,945 \\
\hline 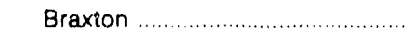 & 8 & 2,705 & 1 & 163 & 9 & 2,868 \\
\hline 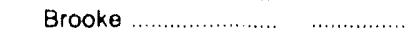 & 1 & 1,181 & 3 & 126 & 4 & 1,307 \\
\hline 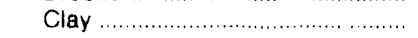 & 4 & 709 & 2 & 12.9 & 6 & 837 \\
\hline 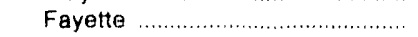 & 8 & 987 & 12 & 881 & 20 & 1,868 \\
\hline Gilmer & 1 & 46 & - & - & 1 & 46 \\
\hline 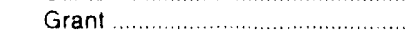 & 4 & 4,088 & 3 & 694 & 7 & 4,782 \\
\hline 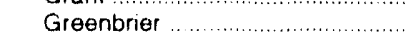 & 8 & 548 & 6 & 346 & 14 & 895 \\
\hline 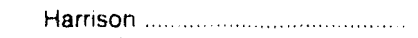 & 6 & 2,401 & 15 & 531 & 21 & 2,933 \\
\hline 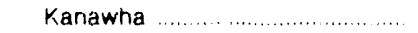 & 13 & 3,144 & 12 & 3,015 & 25 & 6,160 \\
\hline 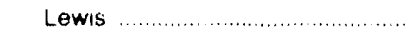 & 3 & 130 & 4 & 326 & 7 & 456 \\
\hline Logan & 64 & 7,577 & 17 & 5,907 & 81 & 13,484 \\
\hline Marion & 4 & 5,220 & 8 & 240 & 12 & 5,460 \\
\hline 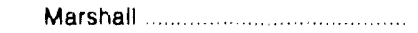 & 3 & 6,740 & - & - & 3 & 6,740 \\
\hline Mason & - & - & 1 & 196 & 1 & 196 \\
\hline 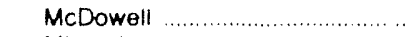 & 64 & 6,317 & 6 & 449 & 70 & 6,766 \\
\hline 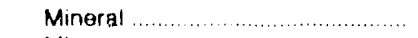 & 1 & 200 & 2 & 246 & 3 & 446 \\
\hline 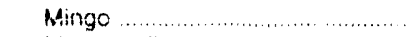 & 37 & 10,381 & 27 & 7,364 & 64 & 17,745 \\
\hline 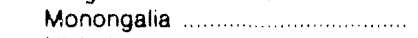 & 14 & 15,917 & 18 & 1,245 & 32 & 17.162 \\
\hline 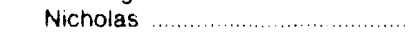 & 26 & 3,120 & 28 & 5,579 & 54 & 8,698 \\
\hline 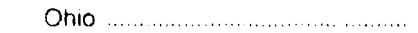 & - & - & 2 & 242 & 2 & 242 \\
\hline
\end{tabular}

Soe Footnotes at end of table 
Table 4. U.S. Coal Production ${ }^{1}$ and Number of Mines by State and County and Type of Mining, 1989 (Continued) (Thousand Short Tons)

\begin{tabular}{|c|c|c|c|c|c|c|}
\hline \multirow{3}{*}{$\begin{array}{l}\text { Coak-Producing } \\
\text { State and County }\end{array}$} & \multicolumn{4}{|c|}{ Type of Mining } & \multicolumn{2}{|c|}{ Total } \\
\hline & \multirow{2}{*}{\multicolumn{2}{|c|}{ Underground }} & \multicolumn{2}{|c|}{ Surfece } & & \multirow{3}{*}{ Production } \\
\hline & & & $; \quad ; \quad \ldots$ & . & Number of Mines & \\
\hline & Number of Mines & Production & Number of Mines & Production & & \\
\hline \multicolumn{7}{|l|}{ Weet Virginie (Continued) } \\
\hline 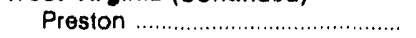 & 16 & 2,601 & 28 & 1,898 & 44 & 4,500 \\
\hline Raleigh & 20 & 7,730 & 2 & 61 & 22 & 7,791 \\
\hline 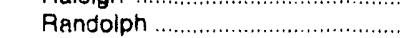 & 9 & 1,582 & 3 & 256 & 12 & 1,838 \\
\hline Tayior & - & 1,00 & 1 & 64 & 1 & 64 \\
\hline 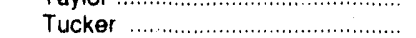 & - & - & 2 & 315 & 2 & 315 \\
\hline 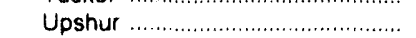 & 2 & 1,551 & 5 & 216 & 7 & 1,768 \\
\hline Wayne & - & 1,00 & 3 & 809 & 3 & 809 \\
\hline 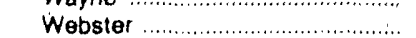 & 8 & 1,082 & 8 & 1,593 & 16 & 2,675 \\
\hline Wyoming & 28 & 8,245 & 4 & 832 & 32 & 8,077 \\
\hline Wyoming & 3 & 1,645 & 25 & 169,913 & 28 & 171,558 \\
\hline 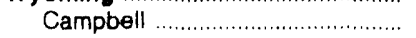 & - & - & 14 & 143,865 & 14 & 143,865 \\
\hline Carbon & 1 & 1,561 & 3 & 3,233 & 4 & 4,794 \\
\hline 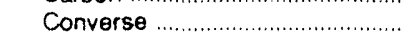 & - & - & 2 & 6,100 & 2 & 6,100 \\
\hline 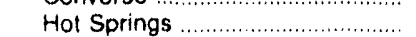 & - & - & 1 & 52 & 1 & 52 \\
\hline Lincoln & - & - & 2 & 4,776 & 2 & 4,776 \\
\hline Sheridan & - & - & 1 & 111 & 1 & 111 \\
\hline 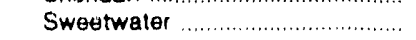 & 2 & 85 & 2 & 11,776 & 4 & 11,860 \\
\hline U.S. Total & 1,426 & 392,618 & 1,395 & 584,929 & 2,821 & 977,547 \\
\hline
\end{tabular}

1 Excludes silt, culm, refuse bank, slurry dam, and dredge production except for Pennsylvania anthracite. Excludes mines producing less than 10,000 short tons of coal during the year.

Note: Total may not equal sum of components because of independent rounding.

Source: Energy Information Administration, Form EIA-7A, "Coal Production Report." 
Table 5. U.S. Coal Production ${ }^{1}$ and Number of Mines by State, Type of Mining, and Mine Production Range, 1989 (Thousard Short Tons)

\begin{tabular}{|c|c|c|c|c|c|c|c|c|c|c|}
\hline \multirow{3}{*}{$\begin{array}{l}\text { Coal-Producing State } \\
\text { and Type of Mining }\end{array}$} & \multicolumn{10}{|c|}{ Mine Production Range } \\
\hline & \multicolumn{2}{|c|}{$\begin{array}{l}500,000 \text { tons } \\
\text { and over }\end{array}$} & \multicolumn{2}{|c|}{$\begin{array}{l}200,000 \text { to } \\
499,999 \text { tons }\end{array}$} & \multicolumn{2}{|c|}{$\begin{array}{l}100,000 \text { to } \\
199,999 \text { tons }\end{array}$} & \multicolumn{2}{|c|}{$\begin{array}{l}50,000 \text { to } \\
99,999 \text { tons }\end{array}$} & \multicolumn{2}{|c|}{$\begin{array}{l}10,000 \text { to } \\
49,999 \text { tons }\end{array}$} \\
\hline & $\begin{array}{c}\text { Number } \\
\text { of } \\
\text { Mines }\end{array}$ & Production & $\begin{array}{c}\text { Number } \\
\text { of } \\
\text { Mines }\end{array}$ & Production & $\begin{array}{c}\text { Number } \\
\text { of } \\
\text { Mines }\end{array}$ & Production & $\begin{array}{c}\text { Number } \\
\text { of } \\
\text { Mines }\end{array}$ & Production & $\begin{array}{c}\text { Number } \\
\text { of } \\
\text { Mines }\end{array}$ & Production \\
\hline 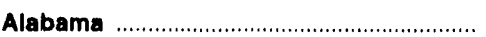 & 15 & 20,997 & 10 & 3,796 & 9 & 1,152 & 14 & 1,082 & 32 & 861 \\
\hline Underground & 9 & 16,199 & - & - & - & - & 1 & 76 & 2 & 31 \\
\hline Surface & 6 & 4,797 & 10 & 3,796 & 9 & 1,152 & 13 & 1,006 & 30 & 830 \\
\hline Alaska & 1 & 1,582 & - & - & - & - & - & - & - & - \\
\hline Surface & 1 & 1,582 & - & - & - & - & - & $\therefore$ & - & - \\
\hline Arlzona & 2 & 11,935 & - & - & - & - & - & - & - & - \\
\hline 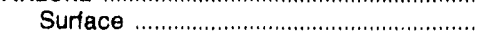 & 2 & 11,935 & - & - & - & - & - & - & - & - \\
\hline Arkansas & - & - & - & - & - & . & - & - & 1 & 39 \\
\hline Surface & - & - & - & - & - & - & - & - & 1 & 39 \\
\hline 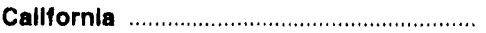 & - & - & - & - & - & - & - & - & 1 & 41 \\
\hline Surface & - & - & - & - & - & - & - & - & 1 & 41 \\
\hline 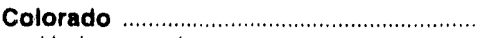 & 10 & 15,158 & 4 & 1,337 & 4 & 523 & - & - & 3 & 98 \\
\hline 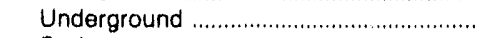 & 7 & 7,186 & 2 & 769 & 4 & 523 & - & - & 1 & 24 \\
\hline Surface & 3 & 7,971 & 2 & 567 & - & - & - & - & 2 & 73 \\
\hline 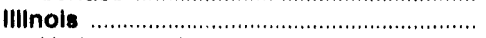 & 38 & 58,172 & 3 & 854 & 1 & 173 & - & - & 3 & 46 \\
\hline 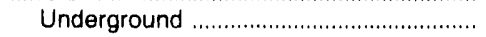 & 23 & 38,487 & 2 & 622 & $i$ & 173 & - & - & 3 & 46 \\
\hline Surface & 15 & 19,685 & 1 & 233 & - & - & - & - & - & - \\
\hline Indiana & 22 & 26,138 & 14 & 5,189 & 11 & 1,690 & 6 & 456 & 5 & 158 \\
\hline Underground & 2 & 1,749 & 2 & 641 & - & - & 2 & 129 & - & - \\
\hline Surface & 20 & 24,389 & 12 & 4,548 & 11 & 1,690 & 4 & 327 & 5 & 158 \\
\hline lowa & - & - & 1 & 312 & - & - & 1 & 79 & 1 & 36 \\
\hline 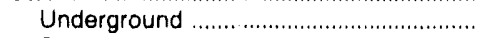 & - & - & - & - & - & - & - & - & $i$ & 36 \\
\hline Surface & - & - & 1 & 312 & - & - & 1 & 79 & - & - \\
\hline 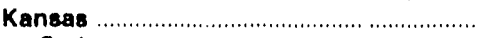 & - & - & 2 & 517 & 2 & 336 & 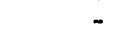 & - & - & - \\
\hline Surface & - & - & 2 & 517 & 2 & 336 & - & - & - & - \\
\hline 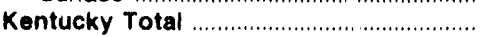 & 78 & 84,185 & 137 & 40,473 & 155 & 21,400 & 163 & 11,842 & 331 & 8,571 \\
\hline Underground & 45 & 47,939 & 78 & 21,814 & 110 & 15,030 & 118 & 8,533 & 194 & 5,275 \\
\hline Surface & 33 & 36,246 & 59 & 18,660 & 45 & 6,369 & 45 & 3,310 & 137 & 3,296 \\
\hline Kentucky, Eastern & 55 & 52,166 & 116 & 33,581 & 142 & 19,743 & 156 & 11,330 & 311 & 8,075 \\
\hline Underground & 32 & 27,518 & 71 & 19,459 & 108 & 14,772 & 115 & 8,282 & 193 & 5,239 \\
\hline Surface & 23 & 24,648 & 45 & 14,122 & 34 & 4,971 & 41 & 3,048 & 118 & 2,836 \\
\hline 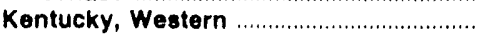 & 23 & 32,019 & 21 & 6,892 & 13 & 1,656 & 7 & 512 & 20 & 496 \\
\hline Underground & 13 & 20,421 & 7 & 2,355 & 2 & 258 & 3 & 251 & 1 & 37 \\
\hline 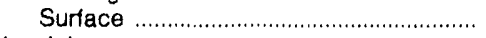 & 10 & 11,598 & 14 & 4,538 & 11 & 1,398 & 4 & 262 & 19 & 460 \\
\hline Loulglana & 1 & 2,880 & - & - & 1 & 103 & - & - & - & - \\
\hline Surfac $\theta$ & 1 & 2,880 & - & - & 1 & 103 & - & - & - & - \\
\hline 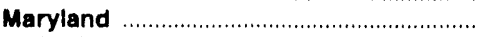 & 1 & 1,834 & 1 & 315 & 4 & 496 & 5 & 392 & 12 & 306 \\
\hline 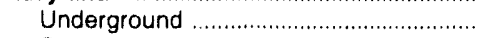 & 1 & 1,834 &. & - & - & - & - & - & - & - \\
\hline Surface & - & - & 1 & 315 & 4 & 496 & 5 & 392 & 12 & 306 \\
\hline 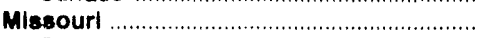 & 2 & 2,891 & - & - & 2 & 322 & 1 & 76 & 3 & 87 \\
\hline Surface & 2 & 2,891 & - & - & 2 & 322 & 1 & 76 & 3 & 87 \\
\hline 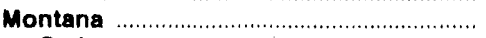 & 6 & 37,438 & 1 & 288 & - & - & - & - & 1 & 16 \\
\hline Surface & 6 & 37,438 & 1 & 288 & - & - & - & - & 1 & 16 \\
\hline 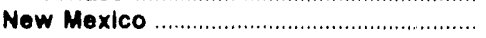 & 5 & 23,202 & 1 & 455 & - & - & - & - & 1 & 45 \\
\hline Underground & - & - & - & - & .- & - & - & - & 1 & 45 \\
\hline Surface & 5 & 23,202 & 1 & 455 & - & - & - & - & - & - \\
\hline North Dakota & 7 & 28,746 & 1 & 489 & 1 & 195 & 2 & 124 & - & - \\
\hline Surface & 7 & 28,746 & 1 & 489 & 1 & 195 & 2 & 124 & - & - \\
\hline Ohlo & 17 & 19,192 & 23 & 7,343 & 25 & 3,528 & 27 & 1,942 & 56 & 1,519 \\
\hline 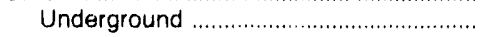 & 7 & 9,802 & 2 & 613 & 2 & 308 & -. & - & 3 & 100 \\
\hline 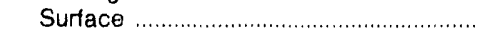 & 10 & 9,390 & 21 & 6,730 & 23 & 3,220 & 27 & 1,942 & 53 & 1,418 \\
\hline Oklahoma & - & - & 3 & 876 & 2 & 277 & 6 & 416 & 5 & 160 \\
\hline 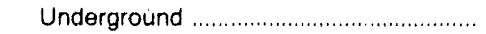 & - & - & - & - & - & - & - & - & 1 & 40 \\
\hline 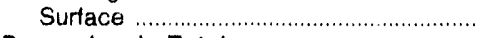 & - & - & 3 & 876 & 2 & 277 & 6 & 416 & 4 & 120 \\
\hline 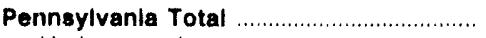 & 30 & 37,245 & 46 & 14,142 & 48 & 6,561 & 80 & 6,152 & 215 & 5,441 \\
\hline Underground & 23 & 31,620 & 15 & 5,235 & 8 & 1,108 & 10 & 846 & 21 & 518 \\
\hline 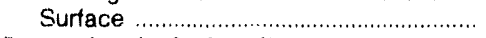 & 7 & 5,625 & 31 & 8,908 & 40 & 5,453 & 70 & 5,306 & 194 & 4,923 \\
\hline Pennaylvania Anthracite ............................... & - & - & 2 & 502 & 5 & 636 & 8 & 625 & 52 & 1,127 \\
\hline Underground & - & - & - & - & - & - & 1 & 93 & 9 & 166 \\
\hline Surface & - & - & 2 & 502 & 5 & 636 & 7 & 532 & 43 & 961 \\
\hline 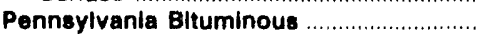 & 30 & 37,245 & 44 & 13,640 & 43 & 5,926 & 72 & 5,528 & 163 & 4,314 \\
\hline 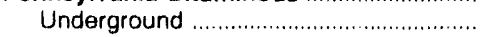 & 23 & 31,620 & 15 & 5,235 & 8 & 1,108 & 9 & 753 & 12 & 352 \\
\hline 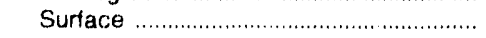 & 7 & 5,625 & 29 & 8,406 & 35 & 4,818 & 63 & 4,775 & 151 & 3,962 \\
\hline
\end{tabular}

See Footnotes at end of table. 
Table 5. U.S. Coal Production ${ }^{1}$ and Number of Mines by State, Type of Mining, and Mine Production Range, 1989 (Continued) (Thousand Short Tons)

\begin{tabular}{|c|c|c|c|c|c|c|c|c|c|c|}
\hline \multirow{3}{*}{$\begin{array}{l}\text { Coal-Pr, ducing State } \\
\text { and Typ : of Mining }\end{array}$} & \multicolumn{10}{|c|}{ Mine Production Range } \\
\hline & \multicolumn{2}{|c|}{$\begin{array}{l}500,000 \text { tons } \\
\text { and over }\end{array}$} & \multicolumn{2}{|c|}{$\begin{array}{c}200,000 \text { to } \\
499,999 \text { tons }\end{array}$} & \multicolumn{2}{|c|}{$\begin{array}{l}100,000 \text { to } \\
199,999 \text { tons }\end{array}$} & \multicolumn{2}{|c|}{$\begin{array}{l}50,000 \text { to } \\
99,999 \text { tons }\end{array}$} & \multicolumn{2}{|c|}{$\begin{array}{c}10,000 \text { to } \\
49,999 \text { tons }\end{array}$} \\
\hline & $\begin{array}{c}\text { Number } \\
\text { of } \\
\text { Mines }\end{array}$ & Production & $\begin{array}{c}\text { Number } \\
\text { of } \\
\text { Mines }\end{array}$ & Production & $\begin{array}{c}\text { Number } \\
\text { of } \\
\text { Mines }\end{array}$ & Production & $\begin{array}{c}\text { Number } \\
\text { of } \\
\text { Mines }\end{array}$ & Froduction & $\begin{array}{c}\text { Number } \\
\text { of } \\
\text { Mines }\end{array}$ & Production \\
\hline 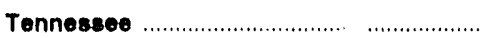 & 1 & 848 & 5 & 1,478 & 16 & 2,245 & 14 & 912 & 32 & 884 \\
\hline Underground & 1 & 848 & 3 & 866 & 12 & 1,676 & 9 & 586 & 23 & 621 \\
\hline 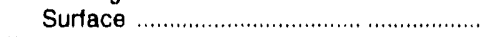 & - & - & 2 & 612 & 4 & 569 & 5 & 326 & 9 & 263 \\
\hline 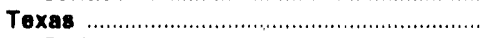 & 10 & 53,075 & 3 & 715 & - & - & 1 & 60 & - & - \\
\hline Surface & 10 & 53,075 & 3 & 715 & - & - & 1 & 60 & - & - \\
\hline 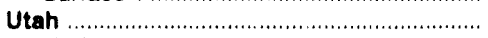 & 9 & 17,203 & 7 & 2,578 & 1 & 192 & 1 & 96 & 2 & 30 \\
\hline 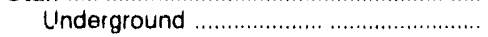 & 9 & 17,203 & 7 & 2,578 & 1 & 192 & 1 & 96 & 2 & 30 \\
\hline Virglnia & 10 & 12,496 & 48 & 13,159 & 57 & 7,824 & 91 & 6,462 & 101 & 2,826 \\
\hline Underground & 9 & 11,982 & 35 & 9,916 & 47 & 6,444 & 77 & 5,497 & 75 & 2,080 \\
\hline Surface & 1 & 513 & 13 & 3,243 & 10 & 1,380 & 14 & 965 & 26 & 746 \\
\hline WashIngton & 2 & 4,898 & - & - & 1 & 141 & - & - & - & - \\
\hline Surtace & 2 & 4,898 & - & - & 1 & 141 & - & - & - & - \\
\hline Wost Virginla & 72 & 88,425 & 100 & 30,286 & 129 & 18,396 & 131 & 9,609 & 227 & 6,127 \\
\hline Underground & 53 & 69,016 & 71 & 21,643 & 90 & 12,948 & 80 & 6,060 & 117 & 3,339 \\
\hline Surface & 19 & 19,409 & 29 & 8,643 & 39 & 5,749 & 51 & 3,549 & 110 & 2,788 \\
\hline Wyoming & 20 & 170,720 & 1 & 362 & 2 & 255 & 2 & 117 & 3 & 104 \\
\hline Underground & 1 & 1,561 & - & 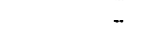 & - & - & 1 & 65 & 1 & 19 \\
\hline 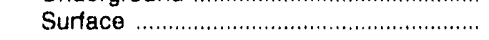 & 19 & 169,159 & 1 & 362 & 2 & 255 & 1 & 52 & 2 & 85 \\
\hline 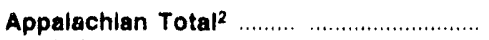 & 201 & 233,202 & 349 & 104,101 & 430 & 60,246 & 518 & 37,883 & 986 & 26,038 \\
\hline Underground & 135 & 168,819 & 197 & 57,732 & 267 & 37,255 & 292 & 21,348 & 434 & 11,928 \\
\hline 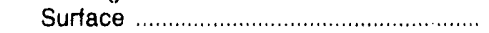 & 66 & 64,383 & 152 & 46,369 & 163 & 22,991 & 226 & 16,535 & 552 & 14,110 \\
\hline Interior Tota| $\left.\right|^{2}$ & 96 & 175,175 & 47 & 15,356 & 32 & 4,557 & 22 & 1,599 & 38 & 1,023 \\
\hline 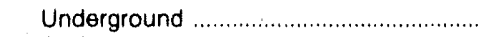 & 38 & 60,657 & 11 & 3,617 & 3 & 432 & 5 & 379 & 6 & 158 \\
\hline 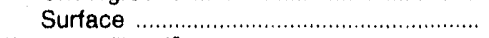 & 58 & 114,518 & 36 & 11,738 & 29 & 4,125 & 17 & 1,220 & 32 & 865 \\
\hline Western Total2 & 62 & 310,882 & 15 & 5,508 & 9 & 1,306 & 5 & 338 & 11 & 333 \\
\hline 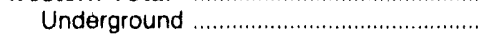 & 17 & 25,950 & 9 & 3,347 & 5 & 715 & 2 & 162 & 5 & 118 \\
\hline 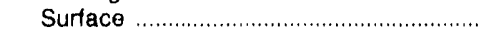 & 45 & 284,932 & 6 & 2,161 & 4 & 591 & 3 & 177 & 6 & 215 \\
\hline 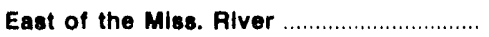 & 284 & 348,531 & 387 & 117,037 & 455 & 63,765 & 531 & 38,851 & 1,014 & 26,739 \\
\hline Underground & 173 & 229,476 & 208 & 61,349 & 270 & $37,6 \& 6$ & 297 & 21,727 & 438 & 12,011 \\
\hline Surface & 111 & 120,055 & 179 & 55,687 & 185 & $26,0 \% 8$ & 2.34 & 17,124 & 576 & 14,728 \\
\hline West of the Miss. River & 75 & 369,728 & 24 & 7,928 & 16 & 2,344 & 14 & 969 & 21 & 655 \\
\hline Underground & 17 & 25,950 & 9 & 3,347 & 5 & 715 & 2 & 162 & 7 & 194 \\
\hline 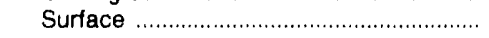 & 58 & 343,778 & 15 & 4,581 & 11 & 1,629 & 12 & 808 & 14 & 461 \\
\hline U.S. Total ........ & 359 & 719,259 & 411 & 124,965 & 471 & 66,108 & 545 & 39,820 & 1,035 & 27,394 \\
\hline Underground & 190 & 255,426 & 217 & 64,696 & 275 & 38,401 & 299 & 21,889 & 445 & 12,205 \\
\hline Surface & 169 & 463,833 & 194 & 60,268 & 196 & 27,707 & 246 & 17,931 & 590 & 15,189 \\
\hline
\end{tabular}

1 Excludes silt, culm, refuse bank, slurry dam, and dradge production except for Pennsylvania antiracite. Excludes mines producing less than 10,000 short tons of coal during the year.

2 For a definition of coal-producing regions, see Appendix C.

Note: Total may not equal sum of components because of independent rounding.

Source: Energy Information Administration, Form ElA-7A, "Coal Production Report." 
Table 6. U.S. Coal Production ${ }^{1}$ by State and Coal Rank, 1989 (Thousand Short Tons)

\begin{tabular}{|c|c|c|c|c|c|c|c|c|c|c|}
\hline \multirow{3}{*}{$\begin{array}{l}\text { Coal-Producing } \\
\text { State }\end{array}$} & \multicolumn{8}{|c|}{ Coal Rank } & \multicolumn{2}{|c|}{ Total } \\
\hline & \multicolumn{2}{|c|}{ Bituminous } & \multicolumn{2}{|c|}{ Subbituminous } & \multicolumn{2}{|c|}{ Llgnito } & \multicolumn{2}{|c|}{ Anthracite } & \multirow[b]{2}{*}{$\begin{array}{l}\text { Number } \\
\text { of Mines }\end{array}$} & \multirow[b]{2}{*}{ Production } \\
\hline & $\begin{array}{l}\text { Number } \\
\text { of MInes }\end{array}$ & Production & $\begin{array}{l}\text { Number } \\
\text { of Mines }\end{array}$ & Production & $\begin{array}{l}\text { Number } \\
\text { of MInes }\end{array}$ & Ploduction & $\begin{array}{c}\text { Number } \\
\text { of Mines }\end{array}$ & Production & & \\
\hline Alabama & 80 & 27,888 & - & - & - & - & - & - & 80 & 27,888 \\
\hline Alaska & - & - & 1 & 1,582 & - & - & - & - & 1 & 1,582 \\
\hline Arizona & 2 & 11,935 & - & - & - & - & - & - & 2 & 11,935 \\
\hline 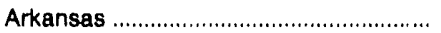 & 1 & 38 & - & - & - & - & - & - & 1 & 39 \\
\hline California & - & - & - & - & 1. & 41 & - & - & 1 & 41 \\
\hline 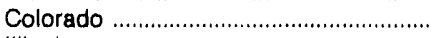 & 18 & 10,592 & 3 & 6,522 & - & - & - & - & 21 & 17,115 \\
\hline 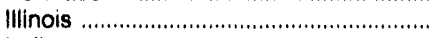 & 45 & 59,246 & - & - & - & - & - & - & 45 & 59,246 \\
\hline Indiana & 58 & 33,631 & - & - & - & - & - & - & 58 & 33,631 \\
\hline 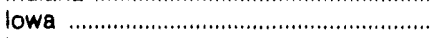 & 3 & 427 & - & - & - & - & - & - & 3 & 427 \\
\hline Kansas & 4 & 854 & - & - & - & - & - & - & 4 & 854 \\
\hline 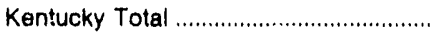 & 864 & 166,472 & - & - & - & - & - & - & 864 & 166,472 \\
\hline 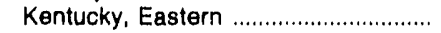 & 780 & 124,895 & - & - & - & - & - & - & 780 & 124,895 \\
\hline 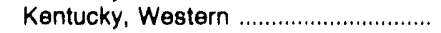 & 84 & 41,577 & - & - & - & - & - & - & 84 & 41,577 \\
\hline Loulsiana & - & - & - & - & 2 & 2,983 & - & - & 2 & 2,983 \\
\hline 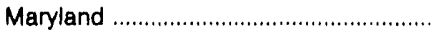 & 23 & 3,343 & - & - & - & - & - & - & 23 & 3,343 \\
\hline 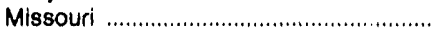 & 8 & 3,377 & -. & - & - & - & - & - & 8 & 3,377 \\
\hline 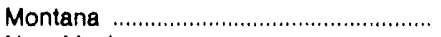 & - & - & 7 & 37,454 & 1 & 288 & - & -. & 8 & 37,742 \\
\hline 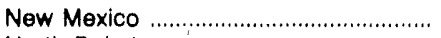 & 5 & 12,486 & 2 & 11,216 & - & - & - & - & 7 & 23,702 \\
\hline 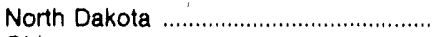 & - & - & - & - & 11 & 29,555 & - & - & 11 & 29,555 \\
\hline Ohio & 148 & 33,525 & - & - & - & - & - & - & 148 & 33,525 \\
\hline 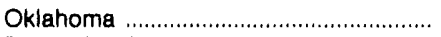 & 16 & 1,728 & - & - & - & - & - & - & 16 & 1,728 \\
\hline 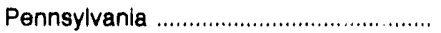 & 352 & 66,653 & - & - & - & - & 67 & 2,889 & $41 \theta$ & 69,542 \\
\hline 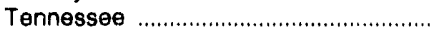 & 68 & 6,367 & - & - & - & - & - & - & 68 & 6,367 \\
\hline 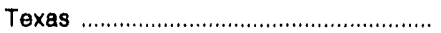 & 2 & 287 & - & - & 12 & 53,562 & - & - & 14 & 53,849 \\
\hline Utah & 20 & 20,099 & - & - & - & - $\quad-$ & - & - & 20 & 20,099 \\
\hline Virginia & 307 & 42,766 & - & - & - & - & - & - & 307 & 42,766 \\
\hline Washington & 1 & 141 & 2 & 4,898 & - & - & - & - & 3 & 5,039 \\
\hline 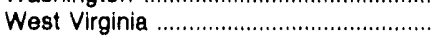 & 659 & 153,143 & - & - & - & - & - & - & 659 & 153,143 \\
\hline 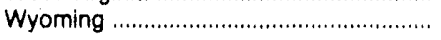 & 5 & 2,060 & 23 & 169,498 & - & - & - & - & 28 & 171,558 \\
\hline Appalachlan Total' .. & 2,417 & 458,580 & - & - & - & - & 67 & 2,889 & 2,484 & 461,469 \\
\hline 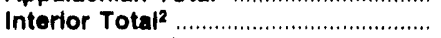 & 221 & 141,165 & - & - & 14 & 56,545 & - & - & 235 & 197,710 \\
\hline 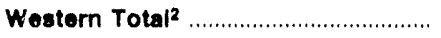 & 51 & 57,313 & 38 & 231,171 & 13 & 29,884 & - & - & 102 & 318,368 \\
\hline $\begin{array}{l}\text { East of the Mise. River } \ldots \ldots \ldots \ldots \ldots \ldots \ldots \\
\text { West of the Miss. Rlver } \ldots \ldots \ldots \ldots \ldots \ldots \ldots\end{array}$ & $\begin{array}{r}2,604 \\
85\end{array}$ & $\begin{array}{r}593,033 \\
64,025\end{array}$ & 38 & $231,17 \overline{1}$ & $\overline{27}$ & 86,429 & $\begin{array}{r}67 \\
-\end{array}$ & 2,889 & $\begin{array}{r}2,671 \\
150\end{array}$ & $\begin{array}{l}595,922 \\
381,625\end{array}$ \\
\hline 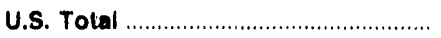 & 2,689 & 657,059 & 38 & 231,171 & 27 & 86,429 & 67 & 2,889 & 2,821 & 977,547 \\
\hline
\end{tabular}

1 Excludes silt, culm, refuse bank, slurry dam, and dredge production except for Pennsylvania anthracite. Excludes mines producing less than 10,000 short tons of coal during the year.

2 For a definition of coal-producing regions, see Appendix C.

Note: Total may not equal sum of components because of independent rounding.

Source: Energy Information Administration, Form ElA-7A, "Coal Production Report." 
Table 7. U.S. Production ${ }^{1}$ of Bituminous Coal by State and Coal Group, ${ }^{2} 1989$ (Thousand Short Tons)

\begin{tabular}{|c|c|c|c|c|c|c|c|c|c|}
\hline \multirow{2}{*}{$\begin{array}{l}\text { Coal-Producing } \\
\text { State }\end{array}$} & \multirow[b]{2}{*}{$\begin{array}{l}\text { Low Volatlle } \\
\text { Production }\end{array}$} & \multirow[b]{2}{*}{$\begin{array}{l}\text { Medlum } \\
\text { Volatile } \\
\text { Production }\end{array}$} & & Hlgh & Volatile Pro & Iuction & & Bltuminous & \multirow{2}{*}{$\begin{array}{l}\text { Total } \\
\text { Bituminous } \\
\text { Production }\end{array}$} \\
\hline & & & $\underset{A}{\text { Subgroup }}$ & $\underset{\text { Subgroup }}{\text { Sub }}$ & $\underset{\mathbf{C}}{\text { Subgroup }}$ & $\begin{array}{c}\text { High } \\
\text { Volatlio } \\
\text { Subgroup } \\
\text { Unknown }\end{array}$ & $\begin{array}{c}\text { High Vola- } \\
\text { tlle } \\
\text { Total }\end{array}$ & $\begin{array}{l}\text { Content/ } \\
\text { Group } \\
\text { Unknown } \\
\text { Production }\end{array}$ & \\
\hline 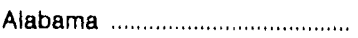 & - & 12,182 & 12,705 & 100 & 41 & 2,860 & 15,706 & - & 27,888 \\
\hline Arizona & - & - & - & 11,935 & - & - & 11,935 & - & 11,835 \\
\hline Arkansas & 39 & - & - & - & - & - & - & - & 39 \\
\hline 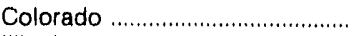 & - & 935 & 1,062 & 429 & 7,649 & 517 & 9,657 & - & 10,592 \\
\hline Hlinois & - & - & 14,716 & 17,552 & 13,217 & 13,760 & 59,246 & - & 59,246 \\
\hline Indiana & - & - & 2,225 & 7,144 & 17,682 & 6,580 & 33,631 & - & 33,631 \\
\hline 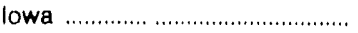 & - & - & - & - & 427 & - & 427 & - & 427 \\
\hline 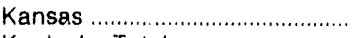 & - & - & - & 277 & 60 & 517 & 854 & - & 854 \\
\hline 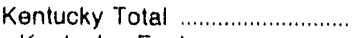 & - & - & 90,193 & 32,296 & 17,320 & 26,663 & 166,472 & - & 166,472 \\
\hline Kentucky, Eastern ........................ & - & - & 79,289 & 12,705 & 12,620 & 20,281 & 124,895 & - & 124,895 \\
\hline 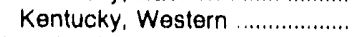 & - & - & 10,904 & 19,591 & 4,700 & 6,382 & 41,577 & - & 41,577 \\
\hline 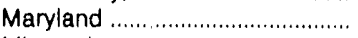 & 3,137 & - & (") & 101 & - & 105 & 206 & - & 3,343 \\
\hline 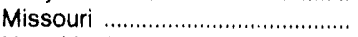 & - & .. & 527 & 2,488 & 136 & 227 & 3,377 & - & 3,377 \\
\hline New Mexico ............................... & - & - & 500 & - & 11,986 & - & 12,486 & - & 12,486 \\
\hline Ohio & - & - & 14,147 & 9,278 & 3,801 & 6,299 & 33,525 & - & 33,525 \\
\hline 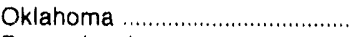 & - & 303 & 834 & 282 & 309 & - & 1,426 & - & 1,728 \\
\hline 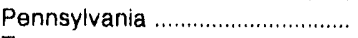 & 7,667 & 18,226 & 36,787 & 3,488 & - & 484 & 40,759 & - & 66,653 \\
\hline 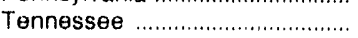 & - & - & 6,357 & - & - & 10 & 6,367 & - & 6,367 \\
\hline 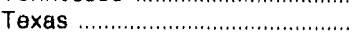 & - & - & - & - & - & 287 & 287 & - & $28 \%$ \\
\hline Utah & - & - & - & 15,681 & 4,418 & - & 20,099 & - & 20,099 \\
\hline 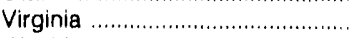 & - & 15,992 & 26,361 & 373 & - & 41 & 26,774 & - & 42,766 \\
\hline Washington $\ldots \ldots \ldots \ldots \ldots \ldots \ldots \ldots \ldots$ & - & - & 141 & - & - & - & 141 & - & 141 \\
\hline 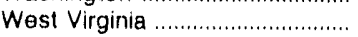 & 19,244 & 6,894 & 125,483 & 196 & 38 & 1,287 & 127,004 & - & 153,143 \\
\hline 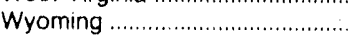 & 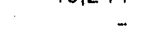 & - & - & - & 2,060 & - & 2,060 & - & 2,060 \\
\hline Appalachlan Total ${ }^{3} \ldots \ldots \ldots \ldots \ldots$ & 30,048 & 53,294 & 301,129 & 26,241 & 16,500 & 31,366 & 375,237 & - & 458,580 \\
\hline Interior Total ${ }^{3}$ & 39 & 303 & 20,207 & 47,333 & 36,531 & 27,753 & 140,823 & - & 141,165 \\
\hline Western Total ${ }^{3} \ldots \ldots \ldots \ldots \ldots \ldots \ldots \ldots$ & - & 935 & 1,703 & 28,045 & 26,114 & 517 & 56,379 & - & 57,313 \\
\hline $\begin{array}{l}\text { East of the Miss. River .......... } \\
\text { West of the Miss. River ......... }\end{array}$ & $\begin{array}{r}30,048 \\
39\end{array}$ & $\begin{array}{r}53,294 \\
1,237\end{array}$ & $\begin{array}{r}328,975 \\
3,064\end{array}$ & $\begin{array}{l}70,527 \\
31,091\end{array}$ & $\begin{array}{l}52,100 \\
27,045\end{array}$ & $\begin{array}{r}58,088 \\
1,548\end{array}$ & $\begin{array}{r}509,690 \\
62,749\end{array}$ & $\overline{-}$ & $\begin{array}{r}593,033 \\
64,025\end{array}$ \\
\hline 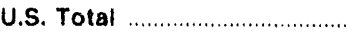 & 30,088 & 54,532 & 332,039 & 101,618 & 79,145 & 59,636 & 572,439 & - & 657,059 \\
\hline
\end{tabular}

1 Excludes silt, culm, refuse bank, slurry dam, and dredge production except for Pennsylvania anthracite. Excludes mines producing less than 10,000 short tons of coal during the year.

2 Refer to the Classification of Coals by Rank table in Appendix $C$ for Coal Group definitions.

3 For a definition of coal-producing regions, see Appendix C.

(") Less than 500 short tons

Note: Total may not equal sum of components because of independent rounding.

Source: Energy Intormation Administration, Form EIA-7A, "Coal Production Report." 
Table 8. U.S. Production ${ }^{1}$ of Subbituminous Coal by State and Coal Group, ${ }^{2} 1989$ (Thousand Short Tons)

\begin{tabular}{|c|c|c|c|c|c|}
\hline \multirow[b]{2}{*}{$\begin{array}{l}\text { Coal-Producing } \\
\text { State }\end{array}$} & \multicolumn{4}{|c|}{ Coal Group } & \multirow[b]{2}{*}{$\begin{array}{c}\text { Total } \\
\text { Subbltuminous } \\
\text { Production }\end{array}$} \\
\hline & $\begin{array}{c}\text { Subbltuminous A } \\
\text { Production }\end{array}$ & $\begin{array}{l}\text { Subbltuminous B } \\
\text { Production }\end{array}$ & $\begin{array}{c}\text { Subbituminous C } \\
\text { Production }\end{array}$ & $\begin{array}{l}\text { Subbltuminous } \\
\text { Group Unknown } \\
\text { Production }\end{array}$ & \\
\hline Alaska & - & - & 1,582 & - & 1,582 \\
\hline Colorado ………....................... & 6,522 & - & - & - & 6,522 \\
\hline 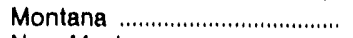 & 16 & 29,720 & 7,718 & - & 37,454 \\
\hline 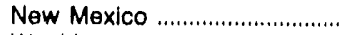 & 11,216 & - & - & - & 11,216 \\
\hline 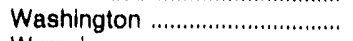 & & - & 4,898 & - & 4,898 \\
\hline 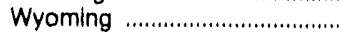 & 8,605 & 13,393 & 147,500 & .. & 169,498 \\
\hline U.S. Total & 26,359 & 43,113 & 161,698 & - & 231,171 \\
\hline
\end{tabular}

1 Excludes silt, culm, refuse bank, slurry dam, and dredge production except for Pennsylvania anthracite. Excludes mines produclng less than 10,000 short tons of coai during the year.

2 Refer to the Classification of Coals by Rank table in Appendix $C$ for Coal Group definitions.

Note: Total may not equal sum of components because of independent rounding.

Source: Energy Information Admiristration, Form EIA-7A, "Coal Production Repürt."

\section{Table 9. U.S. Production ${ }^{1}$ of Lignite Coal by State and Coal Group, ${ }^{2} 1989$} (Thousand Short Tons)

\begin{tabular}{|c|c|c|c|c|}
\hline \multirow[b]{2}{*}{$\begin{array}{l}\text { Coal-Producing } \\
\text { State }\end{array}$} & \multicolumn{3}{|c|}{ Coal Group } & \multirow[b]{2}{*}{$\begin{array}{c}\text { Total } \\
\text { Lignite } \\
\text { Production }\end{array}$} \\
\hline & $\begin{array}{c}\text { Lignite A } \\
\text { Production }\end{array}$ & $\begin{array}{c}\text { Lignite B } \\
\text { Production }\end{array}$ & $\begin{array}{l}\text { Llgnite } \\
\text { Group Unknown } \\
\text { Production }\end{array}$ & \\
\hline California & 41 & - & .. & 41 \\
\hline Louisiana & 2,983 & - & - & 2,983 \\
\hline 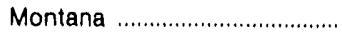 & 288 & - & - & 288 \\
\hline North Dakota .............................. & 29,555 & - & .. & 29,555 \\
\hline 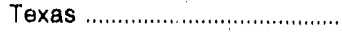 & 47,096 & 6,466 & - & 53,562 \\
\hline U.S. Total & 79,962 & 6,466 & - & 86,429 \\
\hline
\end{tabular}

1 Excludes silt, culm, refuse bank, slurry dam, and dredge production except for Pennsylvania anthracite. Excludes mines producing less than 10,000 short tons of coal during the year.

2 Refer to the Classification of Coals by Rank table in Appendix $\mathrm{C}$ for Coal Group definitions.

Note: Total may not equal sum of components because of independent rounding.

Source: Energy Information Administration, Form EIA-7A, "Coal Production Report." 
Table 10. Pennsylvania Anthracite Production' by County and Coal Group ${ }^{2}, 1989$ (Thousand Short Tons)

\begin{tabular}{|c|c|c|c|c|c|}
\hline \multirow{2}{*}{ County } & \multicolumn{4}{|c|}{ Coal Group } & \multirow{2}{*}{$\begin{array}{c}\text { Total } \\
\text { Anthraclte } \\
\text { Production }\end{array}$} \\
\hline & $\begin{array}{l}\text { Meta-anthracite } \\
\text { Production }\end{array}$ & $\begin{array}{l}\text { Anthractie } \\
\text { Production }\end{array}$ & $\begin{array}{l}\text { Semlanthracite } \\
\text { Production }\end{array}$ & $\begin{array}{l}\text { Anthracite Group } \\
\text { Unknown Production }\end{array}$ & \\
\hline 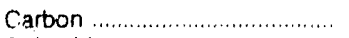 & - & 57 & - & - & 57 \\
\hline Columbia & - & 64 & - & -. & 64 \\
\hline 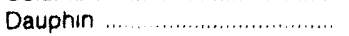 & - & 17 & - & - & 17 \\
\hline Lackawanna ............................ & $\cdots$ & - & - & - & 0 \\
\hline 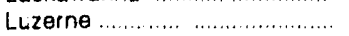 & - & 613 & - & - & 613 \\
\hline Northumberland ......................... & - & 47 & 47 & - & 94 \\
\hline 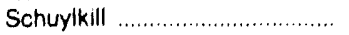 & - & 2,005 &. & - & 2,005 \\
\hline 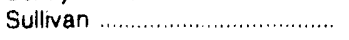 & - & - & 39 & - & 39 \\
\hline 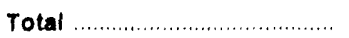 & - & 2,803 & 86 & - & 2,889 \\
\hline
\end{tabular}

1 Includes silt, culm, refuse bank, slurry dam, and dredge production. Excludes mines producing less than 10,000 short tons of coal during the year.

2 Reler to the Classification of Coals by Rank table in Appendix C lor Coal Group definitions.

Note: Total may not equal sum of components because of independent roundirig.

Source: Energy Information Administration, Form EIA-7A, "Coal Production Report."

Table 11. U.S. Coal Production ${ }^{1}$ by Coalbed Thickness and Type of Mining, 1989 (Thousand Short Tons)

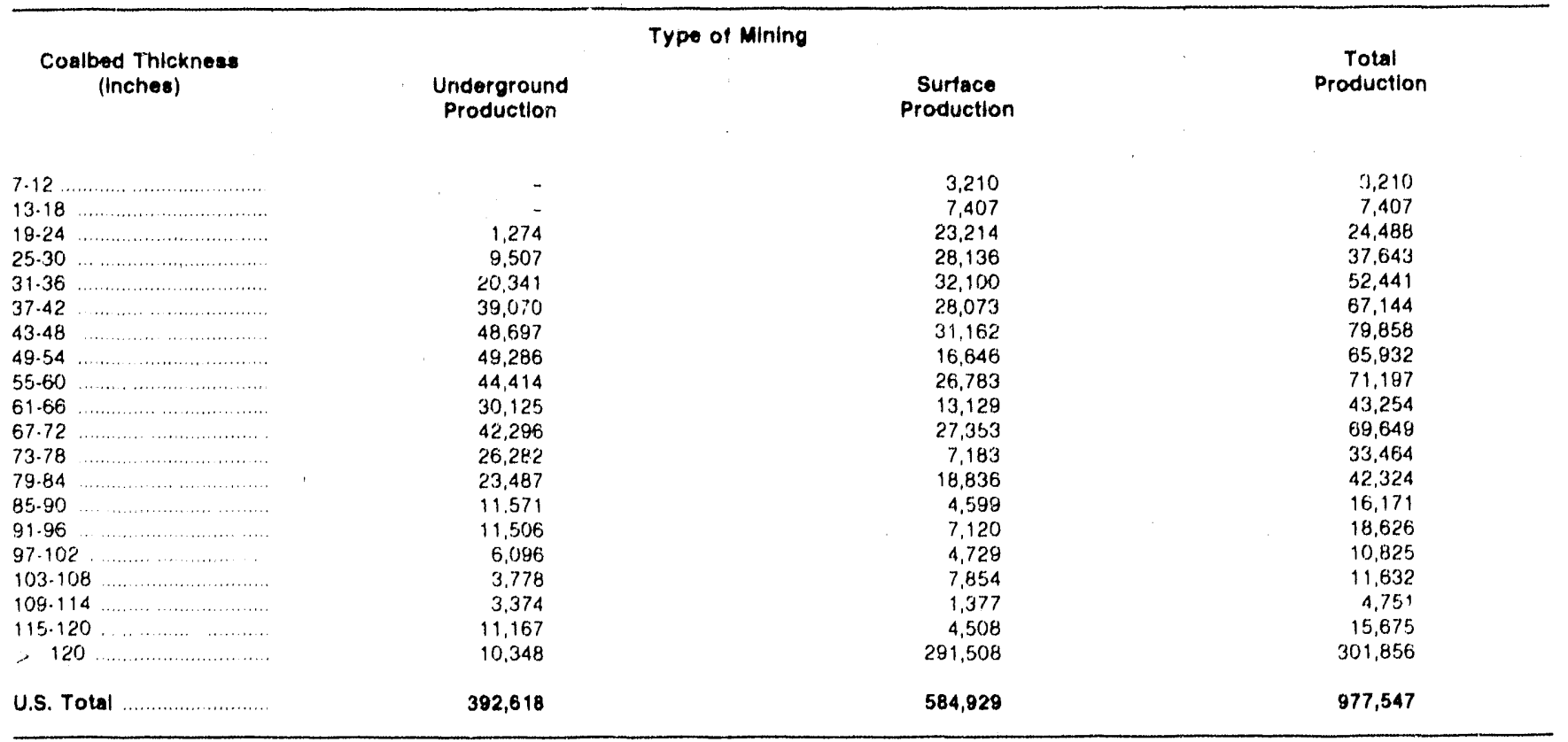

Excludes silt, culm, refuse bank, slurry dam, and dredge production except for Pennsylvania anthracite. Excludes mines producing less than 10,000 short lons of coal during the year.

Nole: Total may not equal sum of components because of independent rounding.

Source: Energy Information Administration, Form EIA.7A "Coal Production Aeport." 
Figure 4. U.S. Coal Production by Coal Rank and Coalbed Thickness, 1980-1989
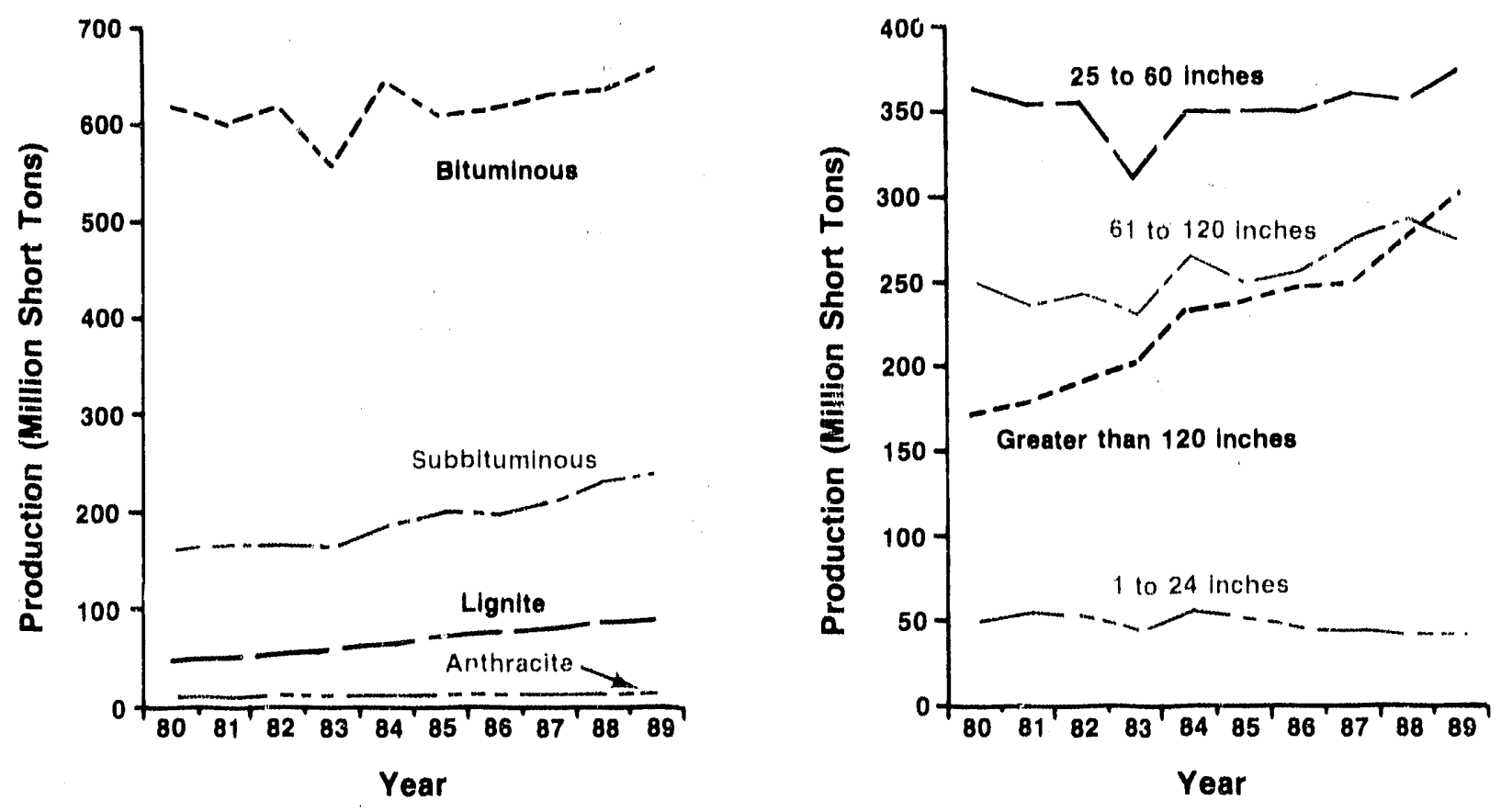

Note: Scale has been enlarged to show detail in the coalbed plot.

Source: Energy Informetion Administration. Form EIA-7A, "Coal Production Report." 


\section{Table 12. U.S. Coal Production ${ }^{1}$ and Coalbed Thickness by Major Coalbeds ${ }^{2}$}

and Type of Mining, 1989

\begin{tabular}{|c|c|c|c|c|c|c|c|c|}
\hline & \multirow{2}{*}{$\begin{array}{l}\text { Coalbed ID Number } \\
\text { Coalbed Name }\end{array}$} & \multicolumn{4}{|c|}{$\begin{array}{c}\text { Production } \\
\text { (Thousand Short Tona) }\end{array}$} & \multicolumn{3}{|c|}{$\begin{array}{c}\text { Thickness } \\
\text { (Inohes) }\end{array}$} \\
\hline & & Underground & Surface & & Total & Average 6 & Low & Hlgh \\
\hline \multicolumn{2}{|c|}{ Major Coulbeds Total ................................. } & 260,116 & 318,227 & & 578,343 & 273.10 & 7 & 1,388 \\
\hline 1699 & 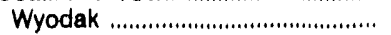 & & 143,305 & & 143,305 & 861.25 & 336 & 1,386 \\
\hline 0036 & 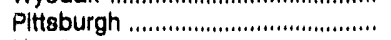 & 53,350 & 6,666 & & 60,016 & 70.24 & 24 & 172 \\
\hline 0484 & 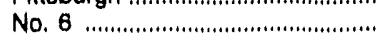 & 34,697 & 15,448 & & 50,145 & 17.74 & 24 & 107 \\
\hline 0489 & No. 9 & 28,723 & 15,929 & & 44,652 & 59.77 & 11 & 100 \\
\hline 0084 & Lower Klttanning ............................. & 9,490 & 17,845 & & 27,335 & 54.42 & 7 & 148 \\
\hline 1569 & 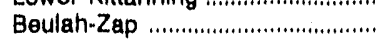 & 0, & 26,105 & & 26,106 & 171.70 & 62 & 210 \\
\hline 0111 & 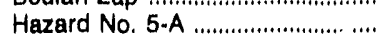 & 9,556 & 15,854 & & 25,410 & 66.62 & 18 & 197 \\
\hline 0071 & 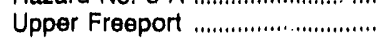 & 15,917 & 8,128 & & 24,046 & 57.50 & 12 & 102 \\
\hline 0135 & 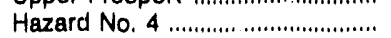 & 18,568 & 2,766 & & 21,334 & 45.07 & 23 & 100 \\
\hline 0168 & 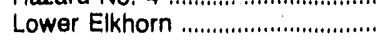 & 19,915 & 1,334 & & 21,250 & 55.03 & 16 & 120 \\
\hline 1808 & Rosebud & - & 20,557 & & 20,557 & 288.38 & 273 & 360 \\
\hline 0157 & Elkhorn No. 1 ............................... & 14,012 & 3,700 & & 17,711 & 57.34 & 15 & 112 \\
\hline 0151 & 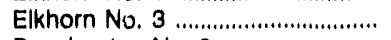 & 14,055 & 2,723 & & 16,778 & 47.61 & 16 & 108 \\
\hline 0344 & 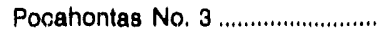 & 15,585 & 35 & & 15,631 & 64.58 & 28 & 66 \\
\hline 0103 & 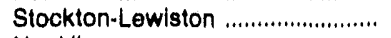 & 7,158 & 8,435 & : & 15,593 & 66.57 & 16 & 120 \\
\hline 0483 & 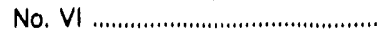 & - & 13,394 & & 13,384 & 61.55 & 24 & 84 \\
\hline 0121 & 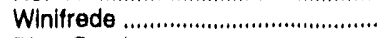 & 6,878 & 5,358 & & 12,236 & 70.65 & 28 & 149 \\
\hline 0280 & 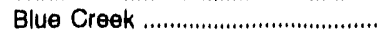 & 10,633 & 873 & & 11,506 & 70.47 & 12 & 96 \\
\hline 0080 & Middle Kittanning ........................... & 1,568 & 9,771 & & 11,339 & 38.13 & 12 & 84 \\
\hline \multicolumn{2}{|c|}{ Other Coalbede } & 132,502 & 266,702 & & 399,204 & 117.74 & 7 & 1,560 \\
\hline \multicolumn{2}{|c|}{ 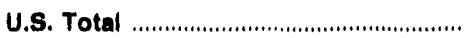 } & 392,618 & 584,929 & & 977,547 & 209.66 & 7 & 1,560 \\
\hline
\end{tabular}

1 Excludes sllt, culm, refuse bank, slurry dam, and dredge production except for Pennsylvanla anthracite. Exoludes mines producing less than 10,000 short tons of coal during the year.

2 A major coalbed is defined here as a coalbed from which 10 million or more short tons were produced during the year. The category "Other Coalbeds" Includes all coalbeds from which less than 10 million short tons were produced during the year. In some regions coalbeds ale characteristically dis. continuous or uncorrelatable from one location to another, and productlon is identified by the geologic formations, coal groups, or voal zones of the native rock where the coalbeds occur. These types of coalbeds are found primarlly in the Rocky Mountaln States and in the Gulf Coast lignite belt. Coalbeds 0 these types are included in "other coalbeds," even though production may exceed 10 million tons, because numerous separate coalbeds contribute to the totals.

3 The coalbed ID number is a unlque code assigned by EIA to each correlated coalbed or to coal-bearing geologlc formations, coal groups, or coal zones.

4he coalbed name given is the name most commonly used in the State having the greatest production from that coalbed. The States having the greatest production for each coalbed are: eastern Kentucky (coalbeds 0111, 0135, 0151, 0157, 0168); West Virginia (0036, 0084, 0103, 0121, 0344); Penn sylvania (0071); Ohlo (0080); western Kentucky (0489); Indiana (0483); Illinols (0484); North Dakota (1580); Montana (1808); Wyoming (1699). In 80me other States where these are major producing beds, the following alternate coalbed names are preferred: 0111, Coalburg (West Virginia); 0121, Hazard No. 5 (Eastern Kentucky), 0151, Jellico (Tennesee), Taggert (Virginia), Cedar Grove (West Virginia); 0157 Upper Standlford (Virginla); 0168 No. 2 Gas (West Virginia); 0484, No. 11 (western Kentucky); 0483, No. 12 (western Kentucky).

5 Average thickness is the bed thickness weighted by bed produotion.

Note: Total may not equal sum of components because of independent rounding.

Source: Energy Information Administration, Form EIA-7A, "Coal Production Report." 
Table 13. U.S. Coal Production' by Mine Produstits Range and Type of Mining, 1988, 1989

(Thousarid Short Tons)

\begin{tabular}{|c|c|c|c|c|c|c|c|c|c|}
\hline \multirow{4}{*}{$\begin{array}{c}\text { MInc Production } \\
\text { Range } \\
\text { (Thousand Short Tons) }\end{array}$} & \multicolumn{6}{|c|}{1989} & \multicolumn{3}{|c|}{1988} \\
\hline & \multicolumn{4}{|c|}{ Type of Mining } & \multicolumn{2}{|c|}{ Total } & \multicolumn{3}{|c|}{ Production } \\
\hline & \multicolumn{2}{|c|}{ Underground } & \multicolumn{2}{|c|}{ Surface } & \multirow[b]{2}{*}{ Production } & \multirow[b]{2}{*}{$\begin{array}{c}\text { Percent } \\
\text { Change } \\
\text { from } \\
\text { Provlous } \\
\text { Year }{ }^{2}\end{array}$} & \multicolumn{2}{|c|}{ Type of Mining } & \multirow[b]{2}{*}{ Total } \\
\hline & Production & $\begin{array}{c}\text { Percent } \\
\text { Change } \\
\text { from } \\
\text { Provlous } \\
\text { Year }{ }^{2}\end{array}$ & Production & $\begin{array}{c}\text { Percent } \\
\text { Changa } \\
\text { from } \\
\text { Provlous } \\
\text { Year }\end{array}$ & & & Underground & Surface & \\
\hline Over 1000 & 198,840 & 7.38 & 417,269 & 5.91 & 616,109 & 6.38 & 185,174 & 393,971 & 579,145 \\
\hline 500 to 1000 & 56,586 & .52 & 46,564 & -0.51 & 103,150 & -4.27 & 56,291 & 51,459 & 107,750 \\
\hline 200 to 500 & 64,696 & 1.11 & 60,268 & 8.64 & 124,965 & 4.61 & 63,987 & 55,473 & 119,460 \\
\hline 100 to 200 & 38,401 & -3.57 & 27,707 & -8.13 & 66,108 & $-5,54$ & 39,823 & 30,160 & 09,983 \\
\hline 50 to 100 & 21,889 & 2.69 & 17,931 & -6.62 & 39,820 & -1.72 & 21,315 & 19,202 & 40,517 \\
\hline 10 to 50 & 12,205 & -12.79 & 15,189 & -3.22 & 27,394 & -7.73 & 13,995 & 16,695 & 29,690 \\
\hline U.S. Totai & 392,618 & 3.16 & 584,929 & 3.35 & 977,547 & 3.28 & 380,584 & 565,961 & 946,545 \\
\hline
\end{tabular}

1 Excludes sllt, culm, refuse bank, slurry dam, and dredge production except for Pennsylvania anthracite. Fxcludes mines producing less than 10,000 short tons of coal during the year.

2 Percent changes are calculated using unrounded data.

Note: Total may not equal sum of components because of independent rounding.

Source: Energy Information Administration, Form ElA-7A, "Coal Production Report."

Table 14. Number of U.S. Coal Mines ${ }^{1}$ by Mine Production Range and Type of Mining, 1988, 1989

\begin{tabular}{|c|c|c|c|c|c|c|c|c|c|}
\hline \multirow{4}{*}{$\begin{array}{c}\text { Mine Production } \\
\text { Range } \\
\text { (Thousand Short Tons) }\end{array}$} & \multicolumn{6}{|c|}{1989} & \multicolumn{3}{|c|}{1988} \\
\hline & \multicolumn{4}{|c|}{ Type of Mining } & \multicolumn{2}{|c|}{ Total } & \multicolumn{3}{|c|}{$\begin{array}{l}\text { Number } \\
\text { of Mines }\end{array}$} \\
\hline & \multicolumn{2}{|c|}{ Underground } & \multicolumn{2}{|c|}{ Surface } & \multirow[b]{2}{*}{$\begin{array}{l}\text { Number } \\
\text { of Mines }\end{array}$} & \multirow[b]{2}{*}{$\begin{array}{c}\text { Percent } \\
\text { Change } \\
\text { from } \\
\text { Prevlous } \\
\text { Year }\end{array}$} & \multicolumn{2}{|c|}{ Type of Mining } & \\
\hline & $\begin{array}{l}\text { Number } \\
\text { of Mines }\end{array}$ & $\begin{array}{c}\text { Percent } \\
\text { Change } \\
\text { from } \\
\text { Prevlous } \\
\text { Year' }\end{array}$ & $\begin{array}{l}\text { Number } \\
\text { of Mines }\end{array}$ & $\begin{array}{c}\text { Percent } \\
\text { Change } \\
\text { from } \\
\text { Previous } \\
\text { Year }{ }^{2}\end{array}$ & & & Underground & Surface & Total \\
\hline Over 1000 & 108 & 5.88 & 101 & 8.60 & 209 & 7.18 & 102 & 93 & 195 \\
\hline 500 to 1000 & 82 & 3.80 & 68 & -9.33 & 150 & -2.60 & 79 & 75 & 154 \\
\hline 200 to 500 & 217 & 2.84 & 194 & 6.01 & 411 & 4.31 & 211 & 183 & 394 \\
\hline 100 to 200 & 275 & 0 & 196 & -5.31 & 471 & -2.28 & 275 & 207 & 482 \\
\hline 50 to 100 & 299 & .67 & 246 & -9.23 & 545 & -4.05 & 297 & 271 & 568 \\
\hline 10 to 50 & 445 & -10.82 & 590 & -5.30 & 1,035 & -7.75 & 499 & 623 & 1,122 \\
\hline U.S. Total & 1,426 & -2.53 & 1,395 & -3.93 & 2,821 & -3.22 & 1,463 & 1,452 & 2,915 \\
\hline
\end{tabular}

1 Excludes silt, culm, refuse bank, slurry dam, and dredge production except for Pennsylvania anthracite. Excludes mines producing less than 10,000 short tons of coal during the year.

2 Percent chariges are calculated using unrounded data.

Source: Energy Information Adrninistration, Form ElA-7A, "Coal Production Report." 
Table 15. U.S. Coal Production ${ }^{1}$ by State and Type of Mining, 1988, 1989 (Thousand Short Tons)

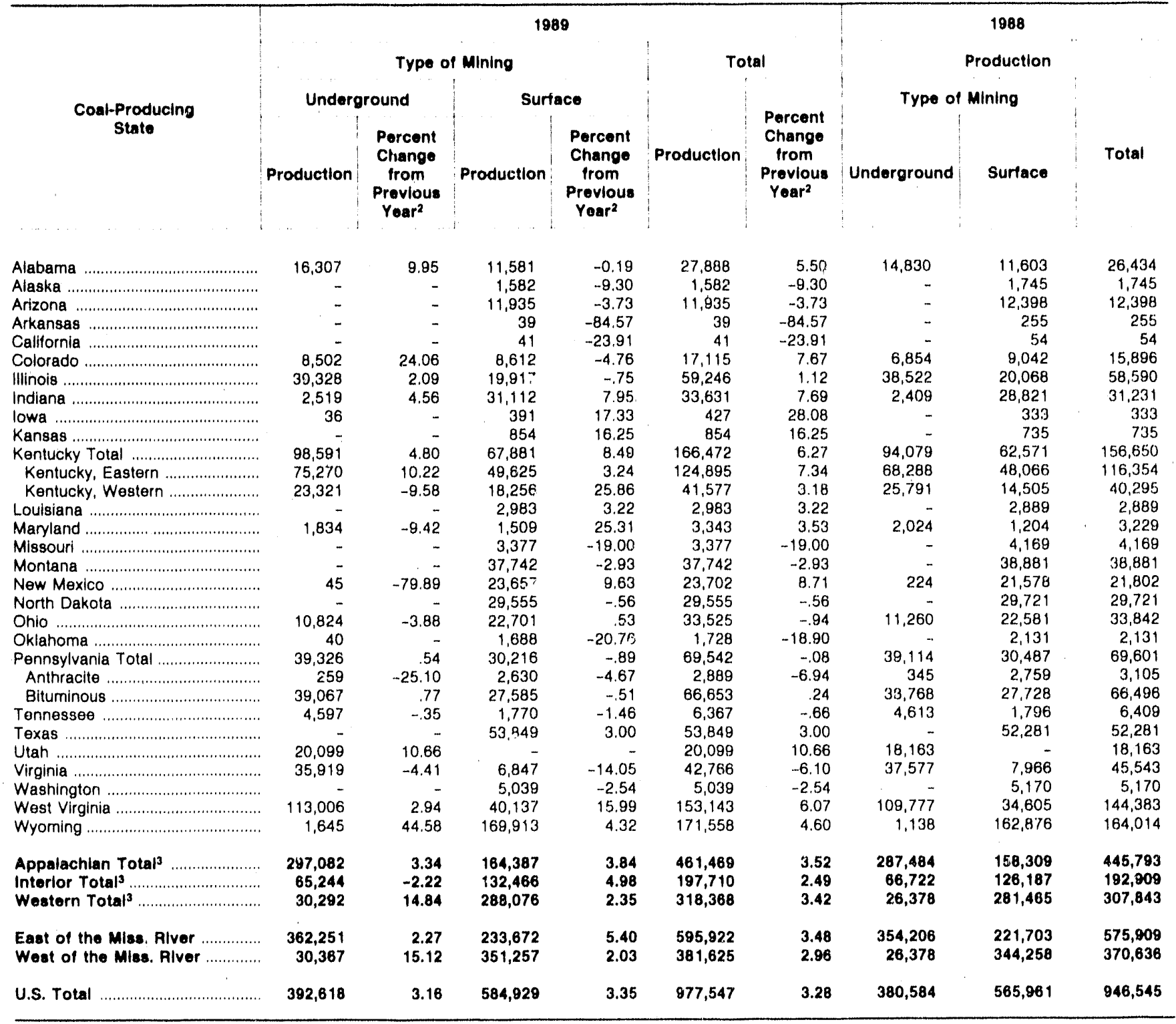

1 Excludes silt, culm, refuse bank, slurry dam, and dredge production except for Pennsylvania anthracite. Excludes mines producing less than 10,000 st.ort tons of coal during the year.

2 Percent changes are calculated : ing unrounded data.

3 For a definition of coal-producing regions, see Appendix. C.

Note: Total may not equal sum of components because of independent rounding.

Source: Energy Information Administration, Form ElA-7A, "Coal Production Report." 
Table 16. Number of U.S. Coal Mines ${ }^{1}$ by State and Type of Mining, 1988, 1989

\begin{tabular}{|c|c|c|c|c|c|c|c|c|c|}
\hline \multirow{4}{*}{$\begin{array}{l}\text { Coat-Producing } \\
\text { State }\end{array}$} & \multicolumn{6}{|c|}{1989} & \multicolumn{3}{|c|}{1988} \\
\hline & \multicolumn{4}{|c|}{ Type of Mining } & \multicolumn{2}{|c|}{ Total } & \multicolumn{3}{|c|}{$\begin{array}{l}\text { Number } \\
\text { of Mines }\end{array}$} \\
\hline & \multicolumn{2}{|c|}{ Underground } & \multicolumn{2}{|c|}{ Surtace } & \multirow[b]{2}{*}{$\begin{array}{l}\text { Number } \\
\text { of Mines }\end{array}$} & \multirow[b]{2}{*}{$\begin{array}{c}\text { Percent } \\
\text { Change } \\
\text { from } \\
\text { Provlous } \\
\text { Year }^{2}\end{array}$} & \multicolumn{2}{|c|}{ Type of Mining } & \multirow[b]{2}{*}{ Total } \\
\hline & $\begin{array}{l}\text { Number } \\
\text { of Mines }\end{array}$ & $\begin{array}{c}\text { Percent } \\
\text { Change } \\
\text { from } \\
\text { Previous } \\
\text { Yoar }^{2}\end{array}$ & $\begin{array}{l}\text { Number } \\
\text { of Mines }\end{array}$ & $\begin{array}{c}\text { Percent } \\
\text { Change } \\
\text { from } \\
\text { Provlous } \\
\text { Year }\end{array}$ & & & Underground & Surface & \\
\hline Alabama & 12 & 0 & 68 & 4.62 & 80 & 3.90 & 12 & 65 & 77 \\
\hline Alaska & - & - & 1 & $\begin{array}{r}4.02 \\
0\end{array}$ & 1 & 0 & - & 1 & 1 \\
\hline Arizona & - & - & 2 & 0 & 2 & 0 & - & 2 & 2 \\
\hline 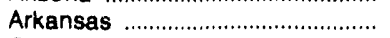 & - & - & 1 & -66.67 & 1 & -66.67 & - & 3 & 3 \\
\hline California ............................... & - & - & 1 & 0 & 1 & 0 & - & 1 & 1 \\
\hline 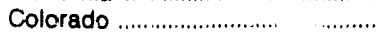 & 14 & 16.67 & 7 & -12.50 & 21 & 5.00 & 12 & 8 & 20 \\
\hline Illinois .............................. & 29 & 3.57 & 16 & -11.11 & 45 & -2.17 & 28 & 18 & 46 \\
\hline Indiana ....................................... & 6 & 20.00 & 52 & -13.33 & 58 & -10.77 & 5 & 60 & 65 \\
\hline 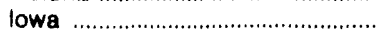 & 1 & - & 2 & -33.33 & 3 & 0 & - & 3 & 3 \\
\hline 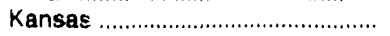 & - & - & 4 & 0 & 4 & 0 & - & 4 & 4 \\
\hline Kentucky Total .......................... & 545 & -5.71 & 319 & -12.12 & 864 & -8.18 & 578 & 363 & 841 \\
\hline Kentucky, Eastern ......................... & 519 & -6.32 & 261 & -14.71 & 780 & -9.30 & 554 & 306 & 860 \\
\hline Kontucky, Western ..................... & 26 & 8.33 & 58 & 1.75 & 84 & 3.70 & 24 & 57 & 81 \\
\hline 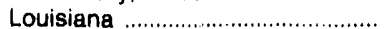 & - & - & 2 & 100.00 & 2 & 100.00 & - & 1 & 1 \\
\hline Maryland ................................... & 1 & -50.00 & 22 & 0 & 23 & -4.17 & 2 & 22 & 24 \\
\hline Missouri .......................................... & - & - & 8 & -20.00 & 8 & -20.00 & - & 10 & 10 \\
\hline Montana ……………………. & - & - & 8 & 0 & 8 & 0 & - & 8 & 8 \\
\hline 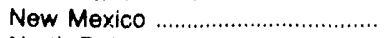 & 1 & 0 & 6 & -25.00 & 7 & -22.22 & 1 & 8 & 9 \\
\hline 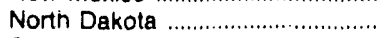 & - & -. & 11 & 10.00 & 11 & 10.00 & - & 10 & 10 \\
\hline 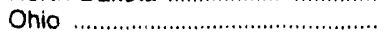 & 14 & 7.69 & 134 & -11.26 & 148 & -9.76 & 13 & 151 & 164 \\
\hline 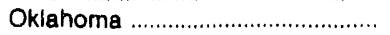 & 1 & - & 15 & -28.57 & 16 & -23.81 & - & 21 & 21 \\
\hline Pennsylvania Total .............................. & 77 & -1.28 & 342 & -3.39 & 419 & -3.01 & 78 & 354 & 432 \\
\hline Anthracite & 10 & 25.00 & 57 & 1.79 & 67 & 4.69 & 8 & 56 & 64 \\
\hline 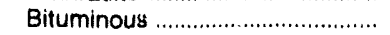 & 67 & -4.28 & 285 & -4.36 & 352 & -4.35 & 70 & 298 & 368 \\
\hline 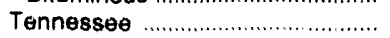 & 48 & -11.11 & 20 & -13.04 & 68 & -11.69 & 54 & 23 & 77 \\
\hline 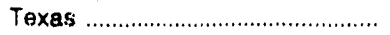 & - & - & 14 & 0 & 14 & 0 & - & 14 & 14 \\
\hline 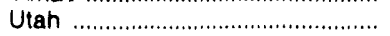 & 20 & 0 & - & - & 20 & 0 & 20 & - & 20 \\
\hline Virginia ...................................... & 243 & -3.18 & 64 & -15.79 & 307 & -6.12 & 251 & 76 & 327 \\
\hline Washington & - & & 3 & 0 & 3 & 0 & - & 3 & 3 \\
\hline 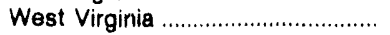 & 411 & 1.23 & 248 & 26.53 & 6.59 & 9.47 & 406 & 196 & 602 \\
\hline 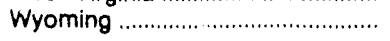 & 3 & 0 & 25 & -7.41 & 28 & -6.67 & 3 & 27 & 30 \\
\hline Appalechlan Total3 & 1,325 & -3.28 & 1,159 & -2.85 & 2,484 & -3.08 & & 1,183 & 2,583 \\
\hline Interlor Totap .................. & 63 & 10.53 & 172 & -9.95 & 235 & -5.24 & 57 & 101 & 248 \\
\hline Western Totals ............................. & 38 & 5.56 & 64 & -5.88 & 102 & -1.92 & 36 & 68 & 104 \\
\hline $\begin{array}{l}\text { East of the Mise. River } \\
\text { West of the Mise. River }\end{array}$ & $\begin{array}{r}1,386 \\
40\end{array}$ & $\begin{array}{l}-2.87 \\
11.11\end{array}$ & $\begin{array}{r}1,285 \\
110\end{array}$ & $\begin{array}{r}-3.24 \\
-11.28\end{array}$ & $\begin{array}{r}2,671 \\
150\end{array}$ & $\begin{array}{r}-3.05 \\
-6.25\end{array}$ & $\begin{array}{r}1,427 \\
36\end{array}$ & $\begin{array}{r}1,328 \\
124\end{array}$ & $\begin{array}{r}2,755 \\
160\end{array}$ \\
\hline U.S. Total & 1,426 & -2.53 & 1,395 & -3.93 & 2,821 & -3.22 & 1,463 & 1,462 & 2,815 \\
\hline
\end{tabular}

1 Excludes silt, culm, refuse bank, slurry dam, and oredge production except for Pennsylvania anthracite. Excludes mines producing less than 10,000 short tons of coal during the year.

Percent changes are calculated using unrounded data.

3 For a definition of coal-producing regions, seo Appendix $C$.

Source: Energy Information Administration, Form EIA-7A, "Coal Production Report." 


\section{Table 17. U.S. Coal Production ${ }^{1}$ by District and Type of Mining, 1988, 1989}

(Thousand Short Tons)

\begin{tabular}{|c|c|c|c|c|c|c|c|c|c|}
\hline \multirow{4}{*}{$\begin{array}{l}\text { Coal-Producing } \\
\text { District }\end{array}$} & \multicolumn{6}{|c|}{1989} & \multicolumn{3}{|c|}{1988} \\
\hline & \multicolumn{4}{|c|}{ Type of Mining } & \multicolumn{2}{|c|}{ Total } & \multicolumn{3}{|c|}{ Production } \\
\hline & \multicolumn{2}{|c|}{ Underground } & \multicolumn{2}{|c|}{ Surface } & \multirow[b]{2}{*}{ Production } & \multirow[b]{2}{*}{$\begin{array}{c}\text { Percent } \\
\text { Change } \\
\text { from } \\
\text { Prevlous } \\
\text { Year }\end{array}$} & \multicolumn{2}{|c|}{ Type of Mining } & \multirow[b]{2}{*}{ Total } \\
\hline & Production & $\begin{array}{l}\text { Percent } \\
\text { Change } \\
\text { from } \\
\text { Provlous } \\
\text { Yoar }\end{array}$ & Production & $\begin{array}{c}\text { Percent } \\
\text { Change } \\
\text { from } \\
\text { Prevlous } \\
\text { Year }{ }^{2}\end{array}$ & & & Underground & Surface & \\
\hline 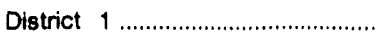 & 21,612 & 0.96 & 24,127 & 5.23 & 45,739 & 3.17 & 21,406 & 22,927 & 44,333 \\
\hline District 2 & 23,576 & 4.49 & 6,223 & -14.76 & 29,799 & -.22 & 22,563 & 7,301 & 29,864 \\
\hline District 3 & 34,859 & -1.07 & 7,633 & 14.66 & 42,492 & 1.43 & 35,234 & 6,657 & 41,891 \\
\hline District 4 & 10,824 & -3.88 & 22,701 & .53 & 33,525 & -.94 & 11,260 & 22,581 & 33,842 \\
\hline 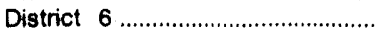 & 7,921 & 15.16 & 368 & 46.96 & 8,289 & 16.28 & 6,878 & 250 & 7,129 \\
\hline 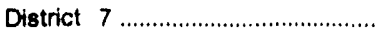 & 14,938 & -1.93 & 524 & 9.49 & 15,462 & -1.58 & 15,231 & 479 & 15,710 \\
\hline 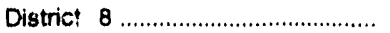 & 166,042 & 4.55 & 87,880 & 5.77 & 253,823 & 4.97 & 158,812 & 83,087 & 241,899 \\
\hline Distric 9 & 23,321 & -0.58 & 18,256 & 25.86 & 41,577 & 3.18 & 25,791 & 14,505 & 40,295 \\
\hline District 10 & 39,328 & 2.09 & 19,917 & -.75 & 59,246 & 1.12 & 38,522 & 20,068 & 58,590 \\
\hline 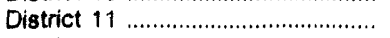 & 2,519 & 4.56 & 31,112 & 7.95 & 33,631 & 7.69 & 2,409 & 28,821 & 31,231 \\
\hline 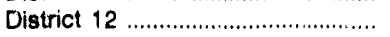 & 36 & - & 391 & 17.33 & 427 & 28.08 & - & 333 & 333 \\
\hline 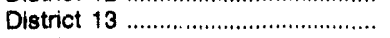 & 17,051 & 8.25 & 12,290 & .26 & 29,351 & 4.75 & 15,752 & 12,267 & 28,020 \\
\hline District 14 & - & - & 342 & -52.98 & 342 & $-52.8 B$ & - & 727 & 727 \\
\hline District 15 & 40 & - & 62,448 & 1.16 & 62,488 & 1.22 & - & 61,733 & 61,733 \\
\hline District 16 & - & - & 27 & -67.25 & 27 & -67.25 & - & 82 & 82 \\
\hline 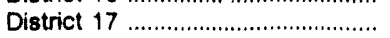 & 8,547 & 20.77 & 9,040 & -5.66 & 17,588 & 5.57 & 7,078 & 9,583 & 16,660 \\
\hline District 18 & . & - & 35,178 & 5.30 & 35,178 & 5.30 & - & 33,408 & 33,408 \\
\hline District 19 & 1,645 & 44.58 & 169,913 & 4.32 & 171,558 & 4.60 & 1,138 & 162,876 & $164,01 \AA$ \\
\hline District 20 & 20,099 & 10.66 & - & - & 20,099 & 10.66 & 18,163 & - & 18,163 \\
\hline District 21 & - & - & 29,555 & -.56 & 29,555 & -.56 & - & 29,721 & 29,721 \\
\hline District 22 & - & - & 37,742 & -2.53 & 37,742 & -2.93 & - & 38,881 & 38,881 \\
\hline District 23 & - & - & 6.621 & -4.25 & 6,621 & -4.25 & - & 6,915 & 6,915 \\
\hline 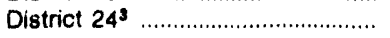 & 259 & -25.10 & 2,630 & -4.67 & 2,889 & -6.94 & 345 & 2,759 & 3,105 \\
\hline 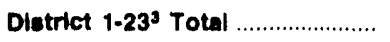 & 392,359 & 3.19 & 582,298 & 3.38 & 974,658 & 3.31 & 380,239 & 563,202 & 943,441 \\
\hline (n) & 392,618 & 3.16 & 584,929 & 3.35 & 877,547 & 3.28 & 380,584 & 565,861 & 946,545 \\
\hline
\end{tabular}

1 Exciudes silt, culm, refuse bank, slurry dam, and dredge production except for Pennsylvania anthracite. Excludes mines producing less than 10,000 short tons of coal during the year.

2 Percent changes are calculated using unrounded data.

3 District 24 is the anthracite-producing district in Penrisylvania. Districts 1.23 represent the total U.3. production of bituminous, subbituminous coal, and lignite. See Appendix C.

Source: Energy Information Administration, Form ElA-7A, "Coal Production Report." 
Table 18. Number of U.S. Coal Mines ${ }^{1}$ by District and Type of Mining, 1988, 1989

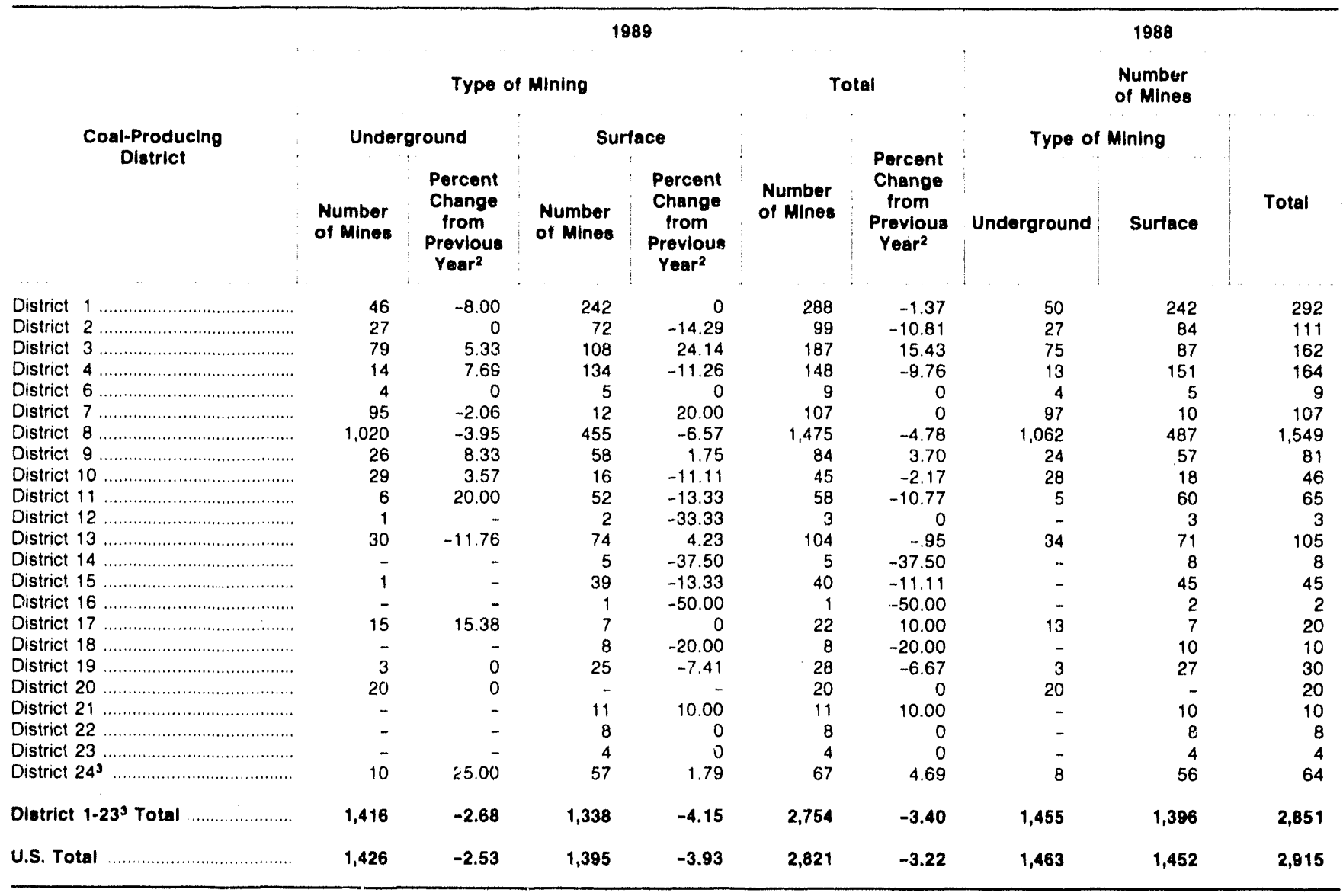

1 Excludes silt, culm, refuse bank, slurry dam, and dredge production except for Pennsylvania anthracite. Excludes mines producing less than 10,000 short tons of coal during the year.

2 Percent changes are calculated using unrounded data.

3 District 24 is the enthracite-producing district in Pennsylvania. Districts 1.23 represent the total II.S. production of bituminous, subbituminous coal, and lignite. See Appendix $C$.

Source: Energy information Administration, Form EIA.7A, "Coal Production Report." 


\section{Average Mine Prices}

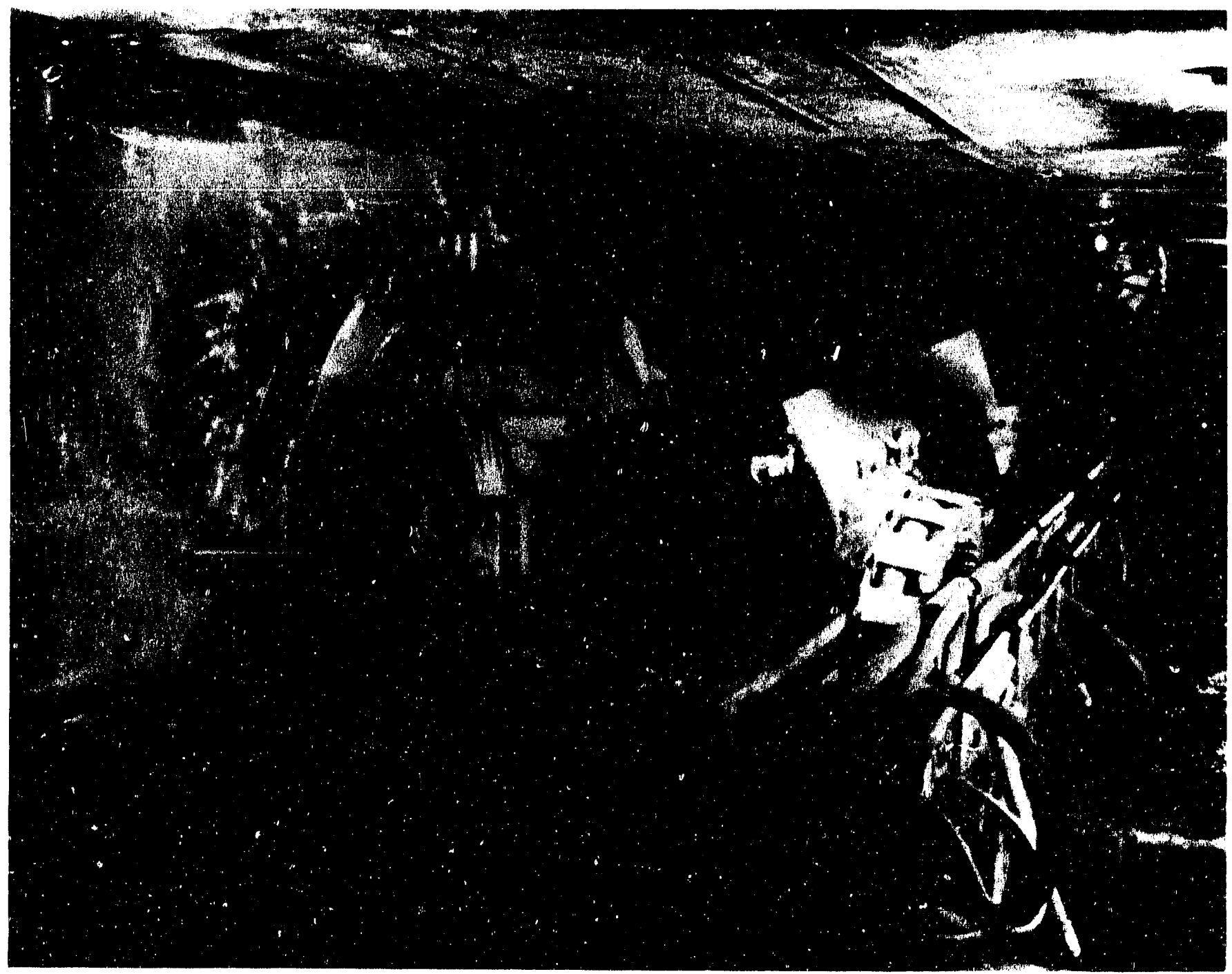

Modern mining machines, such as this longwall-type, spray water to reduce dust to a minimum and to help conserve machine life. 
Figure 5. Average Mine Price of U.S. Coal by Type of Mining and Coal Rank, 1980-1989
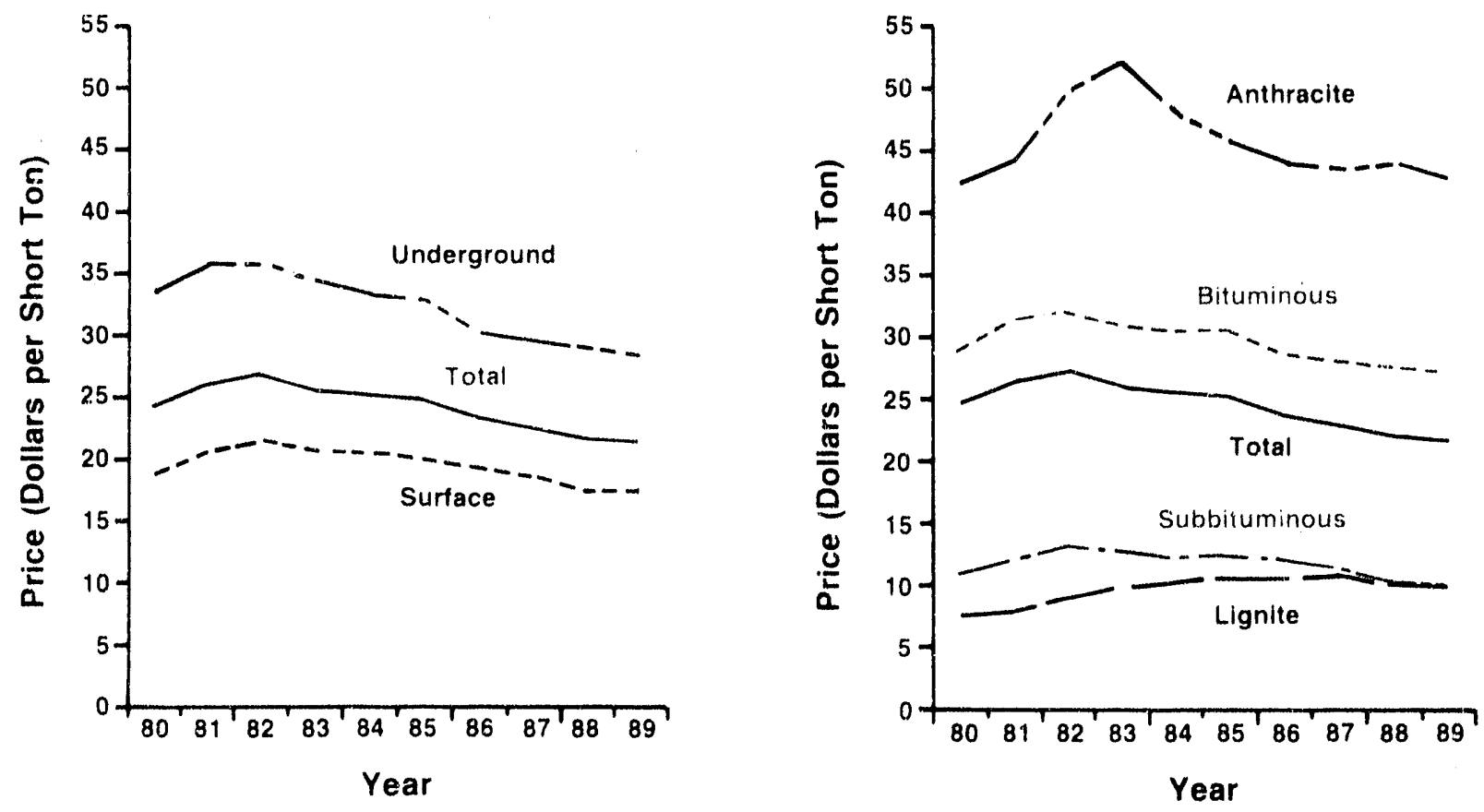

Source: Energy Information Administration, Form ElA-7A, "Coal Production Report." 
Table 19. U.S. Coal Production ${ }^{1}$ and Average Mine Price ${ }^{2}$ by State and Type of Mining, 1989

(Thousand Short Tons, Dollars Per Short Ton)

\begin{tabular}{|c|c|c|c|c|c|c|}
\hline \multirow{3}{*}{$\begin{array}{l}\text { Coal-Producing } \\
\text { State }\end{array}$} & \multicolumn{4}{|c|}{ Type of Mining } & \multicolumn{2}{|c|}{ Total } \\
\hline & \multicolumn{2}{|c|}{ Underground } & \multicolumn{2}{|c|}{ Surface } & \multirow[b]{2}{*}{ Production } & \multirow[b]{2}{*}{$\begin{array}{l}\text { Average Mine } \\
\text { Price }\end{array}$} \\
\hline & Production & $\begin{array}{c}\text { Average Mine } \\
\text { Price }\end{array}$ & Production & $\begin{array}{l}\text { Average Mine } \\
\text { Price }\end{array}$ & & \\
\hline Alabama & 16,307 & 38.96 & 11,581 & 44.31 & 27,888 & 41.18 \\
\hline Alaska & - & - & 1,582 & w & 1,582 & w \\
\hline Arizona & .- & - & 11,935 & $\mathrm{w}$ & 11,935 & $w$ \\
\hline 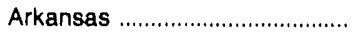 & - & - & 39 & $w$ & 39 & $w$ \\
\hline California ........................................... & - & - & 41 & $w$ & 41 & $w$ \\
\hline Colorado & 8,502 & 26.02 & 8,612 & 21.29 & 17,115 & 23.64 \\
\hline 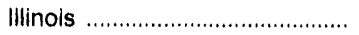 & 39,328 & 28.66 & 19,917 & 27.20 & 59,246 & 28.17 \\
\hline 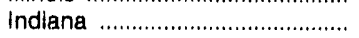 & 2,519 & 27.22 & 31,112 & 23.26 & 33,631 & 23.55 \\
\hline 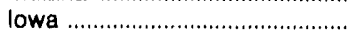 & 36 & $w$ & 391 & w & 427 & w \\
\hline 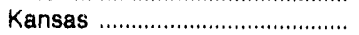 & - & - & 854 & 27.00 & 854 & 27.00 \\
\hline Kentucky Total ............................. & 98,591 & 25.06 & 67,881 & 24.84 & 166,472 & 24.97 \\
\hline Kentucky, Eastern ....................... & 75,270 & 25.69 & 49,625 & 25.96 & 124,895 & 25.80 \\
\hline Kentucky, Western ...................... & 23321 & 23.03 & 18,256 & 21.79 & 41,577 & 22.48 \\
\hline 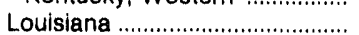 & 20051 & - & 2,883 & w & 2,983 & w \\
\hline 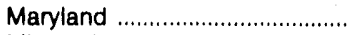 & 1,834 & w & 1,509 & $w$ & 3,343 & 24.73 \\
\hline Missouri ...................................... & - & - & 3,277 & w & 3,377 & $w$ \\
\hline 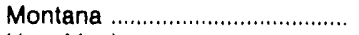 & - & - & 37,742 & 10.27 & 37,742 & 10.27 \\
\hline New Mexico .............................. & 45 & w & 23,657 & w & 23,702 & 23.42 \\
\hline North Dakota ................................ & - & - & 29,555 & 7.36 & 29,555 & 7.36 \\
\hline Ohio & 10,824 & 37.30 & 22,701 & 27.24 & 33,525 & 30.49 \\
\hline Oklahoma & 40 & w & 1,688 & w & 1,728 & 28.46 \\
\hline Pennsylvania Total ........................ & 39,326 & 31.75 & 30,216 & 26.19 & 69,542 & 29.33 \\
\hline Anthracite ................................ & 259 & 37.13 & 2,630 & 43.50 & 2,889 & 42.93 \\
\hline 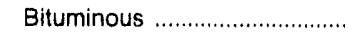 & 39,067 & 31.71 & 27,585 & 24.54 & 66,653 & 28.74 \\
\hline 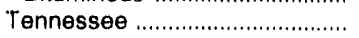 & 4,597 & w & 1,770 & $w$ & 6,367 & 26.98 \\
\hline 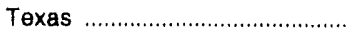 & - & - & 53,849 & 10.91 & 53,849 & 10.91 \\
\hline Utah & 20,099 & 21.46 & - & - & 20,099 & 21.46 \\
\hline 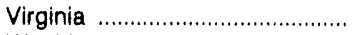 & 35,919 & 28.31 & 6,847 & 24.11 & 42,766 & 27.64 \\
\hline 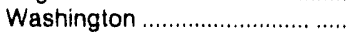 & & - & 5,039 & w & 5,039 & $w$ \\
\hline West Virginia ................................. & 113,006 & 29.49 & 40,137 & 26.42 & 153,143 & 28.69 \\
\hline 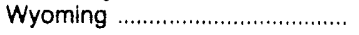 & 1,645 & w & 169,913 & w & 171,558 & 8.63 \\
\hline $\begin{array}{l}\text { Appalaciliail Total } \\
\text { Interlor Total }^{3} \\
\text { Western Total }\end{array}$ & $\begin{array}{r}297,082 \\
65,244 \\
30,292\end{array}$ & $\begin{array}{r}\$ 29.45 \\
26.58 \\
22.55\end{array}$ & $\begin{array}{l}164,387 \\
132,466 \\
288,076\end{array}$ & $\begin{array}{r}\$ 27.45 \\
18.71 \\
11.03\end{array}$ & $\begin{array}{l}461,469 \\
197,710 \\
318,368\end{array}$ & $\begin{array}{r}\$ 28.74 \\
21.31 \\
12.12\end{array}$ \\
\hline $\begin{array}{l}\text { East of the Mlss. River ........ } \\
\text { West of the Miss. River ............. }\end{array}$ & $\begin{array}{r}362,251 \\
30,367\end{array}$ & $\begin{array}{l}28.94 \\
22.54\end{array}$ & $\begin{array}{l}233,672 \\
351,257\end{array}$ & $\begin{array}{l}26.43 \\
11.37\end{array}$ & $\begin{array}{l}595,922 \\
381,625\end{array}$ & $\begin{array}{l}27.95 \\
12.25\end{array}$ \\
\hline U.S. rotal & 392,618 & 28.44 & 584,929 & 17.38 & 977,547 & 21.82 \\
\hline
\end{tabular}

1 Excludes silt, culm, refuse bank, slurry dam, and dredge production except for Pennsylvania anthracite. Excludes mines producing less than 10,000 short tons of coal during the year.

2 Average Mine Price is calculated by dividing the total free on board (f.o.b.) mine value of the coal produced by the total production.

3 For a definition of coal-producing regions, see Appendix C.

w Withheld to avoid disclosure of individual company data.

Note: Total may not equal sum of components because of independent rounding.

Source: Energy Information Administratiol Form EIA-7A, "Coai Production Report." 


\section{Table 20. U.S. Coal Production ${ }^{1}$ and Average Mine Price ${ }^{2}$ by District and Type of Mining, 1989}

(Thousand Short Tons, Dollars Per Short Ton)

\begin{tabular}{|c|c|c|c|c|c|c|}
\hline \multirow{3}{*}{$\begin{array}{c}\text { Coal-Producing } \\
\text { Dlstrict }\end{array}$} & \multicolumn{4}{|c|}{ Type of : *ining } & \multicolumn{2}{|c|}{ Total } \\
\hline & \multicolumn{2}{|c|}{ Underground } & \multicolumn{2}{|c|}{ Surtace } & \multirow[b]{2}{*}{ Production } & \multirow[b]{2}{*}{$\begin{array}{c}\text { Average Mine } \\
\text { Price }\end{array}$} \\
\hline & Production & $\begin{array}{l}\text { Average Mine } \\
\text { Price }\end{array}$ & Production & $\begin{array}{c}\text { Average Mine } \\
\text { Price }\end{array}$ & & \\
\hline 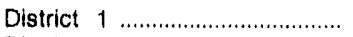 & 21,612 & $w$ & 24,127 & $w$ & 45,739 & 27.66 \\
\hline 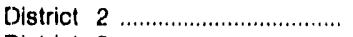 & 23,576 & 31.63 & 6,223 & 26.51 & 29,799 & 30.56 \\
\hline 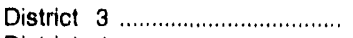 & 34,859 & 27.44 & 7,633 & 21.54 & 42,492 & 26.38 \\
\hline 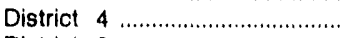 & 10,824 & 37.30 & 22,701 & 27.24 & 33,525 & 30.49 \\
\hline District 6 & 7,921 & $w$ & 368 & $w$ & 8,289 & $w$ \\
\hline District 7 & 14,938 & 30.06 & 524 & 29.96 & 15,462 & 30.06 \\
\hline District 8 & 166,012 & 27.53 & 87,880 & 26.37 & 253,923 & 27.13 \\
\hline District 9 & 23,321 & 23.03 & 18,256 & 2.1 .79 & 41,577 & 22.48 \\
\hline 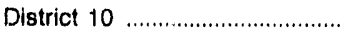 & 39,328 & 28.66 & 19,917 & 27.20 & 59,246 & 28.17 \\
\hline District 11 & 2,519 & 27.22 & 31,112 & 23.26 & 33,631 & 23.55 \\
\hline 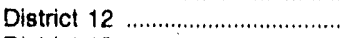 & 36 & $w$ & 391 & $w$ & 427 & $w$ \\
\hline 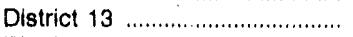 & 17,051 & 38.22 & 12,299 & 43.22 & 29,351 & 40.32 \\
\hline District 14 & - & - & 342 & $w$ & 342 & w \\
\hline District 15 & 40 & $w$ & 62,448 & $w$ & 62,488 & 12.80 \\
\hline 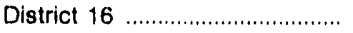 & - & - & 27 & $w$ & 27 & $w$ \\
\hline District 17 & 8,547 & 26.02 & 9,040 & 21.59 & 17,588 & 23.74 \\
\hline 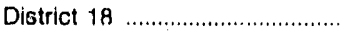 & - & - & 35,178 & 21.82 & 35,178 & 21.82 \\
\hline 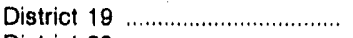 & 1,645 & w & 169,913 & $w$ & 171,558 & 8.63 \\
\hline District $20 \ldots \ldots \ldots \ldots \ldots \ldots \ldots \ldots \ldots \ldots \ldots \ldots \ldots \ldots$ & 20,099 & 21.46 & - & - & 20,099 & 21.46 \\
\hline District 21 & - & - & 29,555 & 7.36 & 29,555 & 7.36 \\
\hline 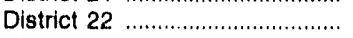 & - & - & 37,742 & 10.27 & 37,742 & 10.27 \\
\hline District 23 & - & - & 6,621 & $w$ & $\$, 621$ & $w$ \\
\hline 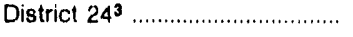 & 259 & 37.13 & 2,630 & 43.50 & 2,889 & 42.93 \\
\hline Dlatrict $\mathbf{1 - 2 3}^{3}$ Total ................... & 392,359 & $\$ 28.44$ & 582,298 & $\$ 17.27$ & 974,658 & $\$ 21.76$ \\
\hline 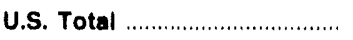 & 392,618 & 28.44 & 584,829 & 17.38 & 877,547 & 21.82 \\
\hline
\end{tabular}

1 Excludes silt, culm, refuse bank, slurry dam, and dredge production except for Pennsylvania anthracite. Excludes mines producing less than 10,000 short tons of coal during the year.

2 Average Mine Price is calculated by dividing the total free on board (f.o.b.) mine value of the coal produced by the total production.

3 District 24 is the anthracite-producing district in Pennsylvania. Districts 1.23 represent the total U.S. production nf bituminous, subbituminous coal, and lignite. See Appendix C.

Withheld to avoid disclosure of individual company data.

Note: Total may not equal sum of components because of independent rounding.

Source: Energy Information Administration, Form ElA-7A, "Coal Production Report." 


\section{Table 21. U.S. Coal Production ${ }^{1}$ and Average Mine Price ${ }^{2}$ by State and Disposition, 1989}

(Thousand Short Tons, Dollars Per Short Ton)

\begin{tabular}{|c|c|c|c|c|c|c|}
\hline \multirow{3}{*}{$\begin{array}{c}\text { Coal-Producing } \\
\text { State }\end{array}$} & \multicolumn{4}{|c|}{ Disposition } & \multicolumn{2}{|c|}{ Total } \\
\hline & \multicolumn{2}{|c|}{ Open Market ${ }^{3}$} & \multicolumn{2}{|c|}{ Captive } & \multirow[b]{2}{*}{ Production } & \multirow[b]{2}{*}{$\begin{array}{l}\text { Average Mine } \\
\text { Price }\end{array}$} \\
\hline & Production & $\begin{array}{l}\text { Average Mlne } \\
\text { Price }\end{array}$ & Production & $\begin{array}{c}\text { Average Mine } \\
\text { Price }\end{array}$ & & \\
\hline Alabama & 26,997 & $w$ & 891 & $w$ & 27,888 & 41.18 \\
\hline 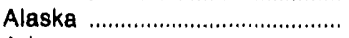 & 1,581 & $w$ & 2 & $w$ & 1,582 & w \\
\hline Arizona & 11,935 & $w$ & - & - & 11,935 & $w$ \\
\hline Arkansas & 39 & $w$ & - & - & 39 & $w$ \\
\hline 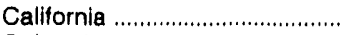 & - & - & 41 & $w$ & 41 & $w$ \\
\hline 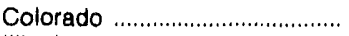 & 16,989 & $w$ & 125 & w & 17,115 & 23.64 \\
\hline 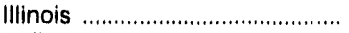 & 59,183 & 28.17 & 53 & 26.93 & 59,246 & 28.17 \\
\hline Indiana & 33,631 & 23.55 & $(*)$ & - & 33,631 & 23.55 \\
\hline 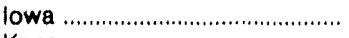 & 427 & $w$ & - & - & 427 & $w$ \\
\hline 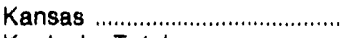 & 854 & 27.00 & - & - & 854 & 27.00 \\
\hline 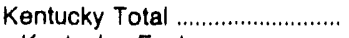 & 163,499 & 24.89 & 2,973 & 29.20 & 166,472 & 24.97 \\
\hline Kentucky, Eastern ...................... & 123,293 & 25.70 & 1,602 & 33.42 & 124,895 & 25.80 \\
\hline Kentucky, Western .................. & 40,205 & 22.42 & 1,371 & 24.27 & 41,577 & 22.48 \\
\hline 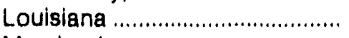 & 103 & $w$ & 2,880 & $w$ & 2,983 & $w$ \\
\hline 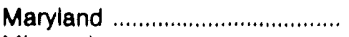 & 3,254 & $w$ & 88 & $w$ & 3,343 & 24.73 \\
\hline 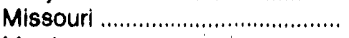 & 987 & w & 2,390 & $w$ & 3,377 & $w$ \\
\hline 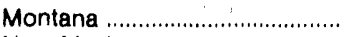 & 33,149 & 10.50 & 4,593 & 8.64 & 37,742 & 10.27 \\
\hline New Mexico ............................... & $23 ; 702$ & 23.42 & - & - & 23,702 & 23.42 \\
\hline North Dakota ............................... & 26,253 & 6.93 & 3,302 & 10.78 & 29,555 & 7.36 \\
\hline 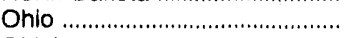 & 25,349 & 26.59 & 8,176 & 42.58 & 33,525 & 30.49 \\
\hline 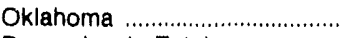 & 1,728 & 28.46 & - & - & 1,728 & 28.46 \\
\hline Pennsylvania Total ...................... & 63,145 & 28.41 & 6,397 & 38.43 & 69,542 & 29.33 \\
\hline 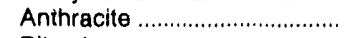 & 2,781 & $w$ & 108 & $w$ & 2,889 & 42.93 \\
\hline 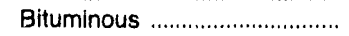 & 60,365 & $w$ & 6,288 & $w$ & 66,653 & 28.74 \\
\hline 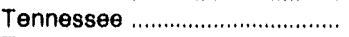 & 6,226 & $w$ & 141 & $w$ & 6,367 & 26.98 \\
\hline 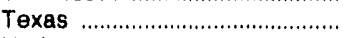 & 11,157 & 11.89 & 42,692 & 10.65 & 53,849 & 10.91 \\
\hline 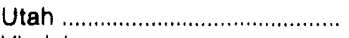 & 13,115 & 23.45 & 6,984 & 17.72 & 20,099 & 21.46 \\
\hline 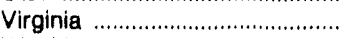 & 42,748 & 27.64 & 19 & 19.34 & 42,766 & 27.64 \\
\hline 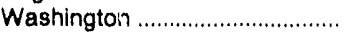 & 141 & $w$ & 4,898 & $w$ & 5,039 & $w$ \\
\hline 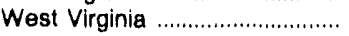 & 144,232 & 28.26 & 8,911 & 35.63 & 153,143 & 28.69 \\
\hline 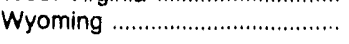 & 167,194 & 8.54 & 4,364 & 12.16 & 171,558 & 8.63 \\
\hline $\begin{array}{l}\text { Appalachian Total }{ }^{5} \ldots \ldots \ldots \ldots \ldots \\
\text { Interior Total }{ }^{6} \ldots \ldots \ldots \ldots \ldots \ldots \ldots \ldots \\
\text { Western Total }\end{array}$ & $\begin{array}{l}435,245 \\
148,324 \\
294,059\end{array}$ & $\begin{array}{r}\$ 28.17 \\
24.27 \\
11.86\end{array}$ & $\begin{array}{l}26,224 \\
49,386 \\
24,309\end{array}$ & $\begin{array}{r}\$ 38.27 \\
12.43 \\
15.36\end{array}$ & $\begin{array}{l}461,469 \\
197,710 \\
318,368\end{array}$ & $\begin{array}{r}528.74 \\
21.31 \\
12.12\end{array}$ \\
\hline $\begin{array}{l}\text { East of the Mles. Rlver ......... } \\
\text { West of the Mles. Rlver ....... }\end{array}$ & $\begin{array}{l}568,274 \\
309,354\end{array}$ & $\begin{array}{l}27.49 \\
12.04\end{array}$ & $\begin{array}{l}27,648 \\
72,271\end{array}$ & $\begin{array}{l}37.55 \\
13.18\end{array}$ & $\begin{array}{l}595,922 \\
381,625\end{array}$ & $\begin{array}{l}27.85 \\
12.25\end{array}$ \\
\hline 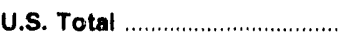 & 877,628 & 22.04 & 99,919 & 19.92 & 977,547 & 21.82 \\
\hline
\end{tabular}

1 Excludes silt, culm, refuse bank, slurry dam, and dredge production except for Pennsylvania anthracite. Excludes mines producing less than 10,000 short tons of coal during the year.

2 Average Mine Price is calculated by dividing the total free on board (f.o.b.) mine value of the coal produced by the total production

3 Open Market includes all coal sold on the open market to other coal companies or consumers.

4 Captive includes all coal used by the producing company or sold to affiliated or parent companies.

- For a definition of coal-producing regions, see Appendix $\mathrm{C}$

(") Less than 500 short tons.

withheld to avoid disclosure of individual company data.

Note: Total may not equal sum of components because of independent rounding

Source: Energy Information Administration, Form ElA-7A, "Coal Production Report." 


\section{Table 22. U.S. Coal Production ${ }^{1}$ and Average Mine Price ${ }^{2}$ by District and Disposition, 1989}

(Thousand Short Tons, Dollars Per Short Ton)

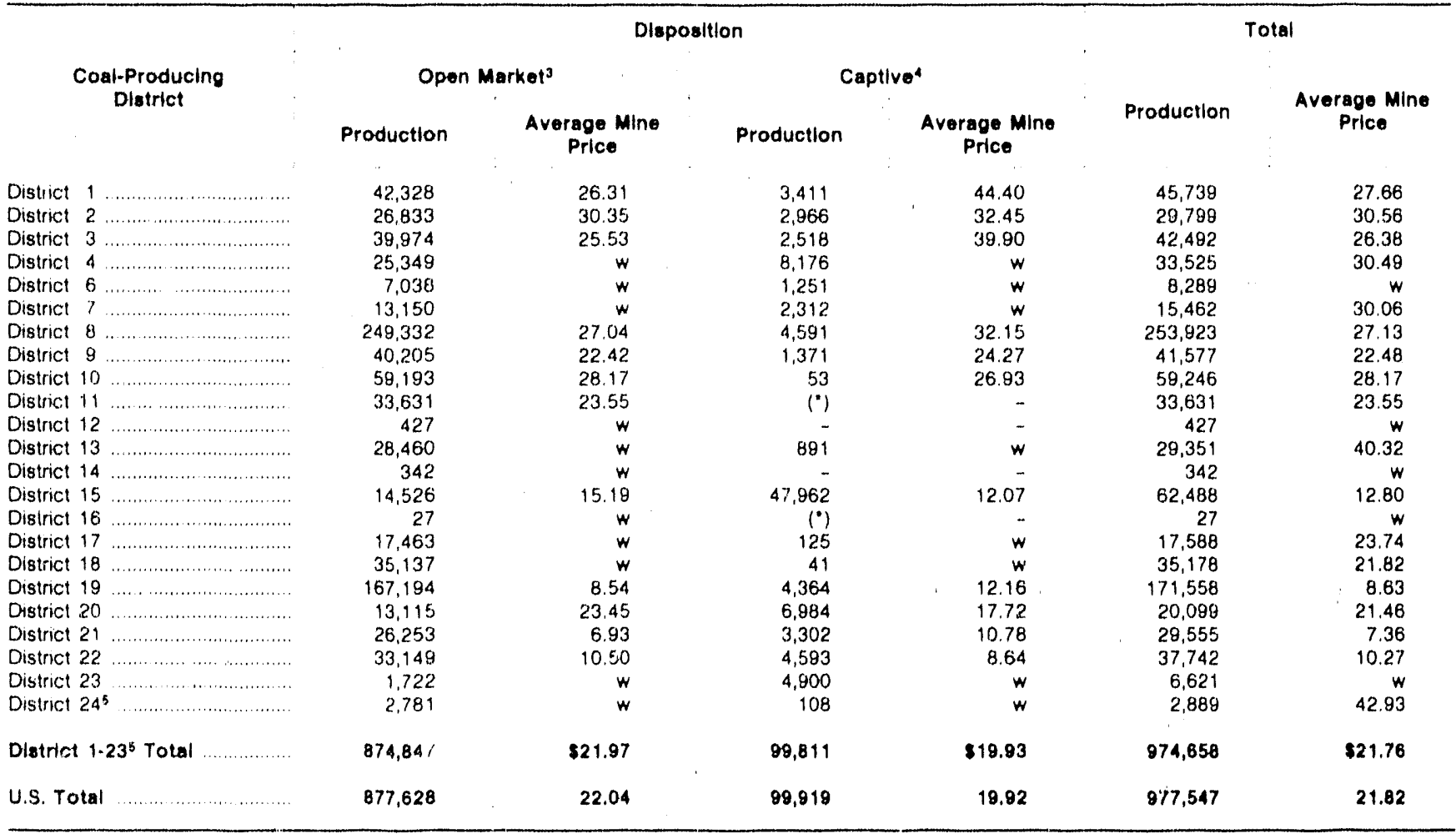

1 Excludes silt, culm, refuse bank, slurry darn, and dredge production except for Pennsylvaria anthracite. Excludes mines producing less than 10,000 short toris of coal during the year.

2 Average Mine Price is calculated by dividing the total free on board (f.o.b.) mine value of the coal produced by the total production.

3 Dpen Markel includes all coal sold on the open market to other coal cornparies or consumers.

4 Captive includes all coal used by the producing company or sold to aftiliated or parent companies.

5 District 24 is the anthracite-producing district in Pennsylvania. Districts 1.23 represent the total U.S. production of bituminous, subbituminous coal, and lignite. Ste Appendix C

(") Less than 500 short tons.

Withheld to avoid disclosure of individual company data

Note: Total may not equal sum of components because of independent rounding

Source Energy iniormation Adrriristration, Form ElA.7A, "Coal Production Repon." 
Table 23. U.S. Coal Production ${ }^{1}$, Number of Mines and Average Mine Price ${ }^{2}$ by State and County, 1989

(Thousand Short Tons, Dollars Per Short Ton)

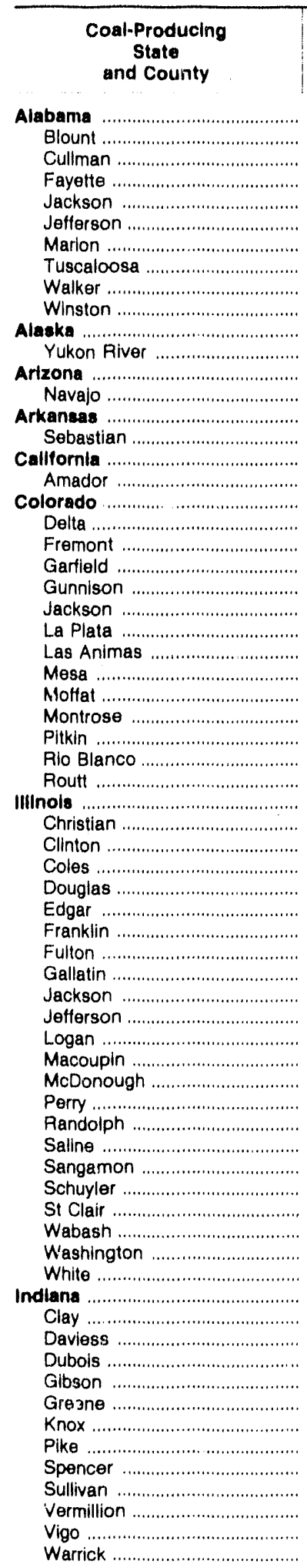

Number of Mines

80

5
1

1

14

7
20
25

Winston

Yukon Piver

Arlzona

Navajo

Sebastian

Callfornia

Amador

Delta

Fremont

Gunnison

Jackson

Las Anima

Mess

Montrose

Routt

Christian

Clinton

Douglas

Fulton

Gallatin

Jefferson

Logan

Randolph

chuyler

W'ashington

White

Davies

Dubois

Greane

Knox

Spencer

Sullivan

Vigo .......

Warrick

See Footnotes at end of table.

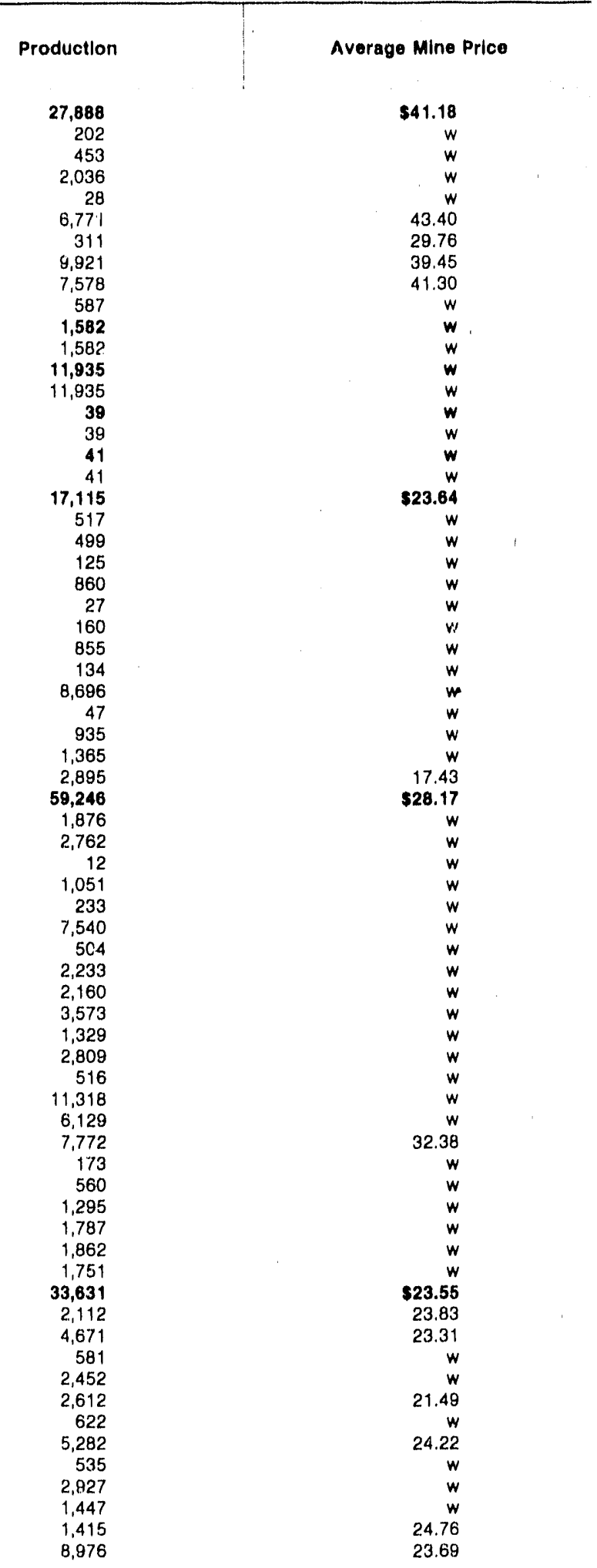


Table 23. U.S. Coal Production ${ }^{1}$, Number of Mines and Average Mine Price ${ }^{2}$ by State and Couinty, 1989 (Continued)

(Thousand Short Tons, Dollars Per Short Ton)

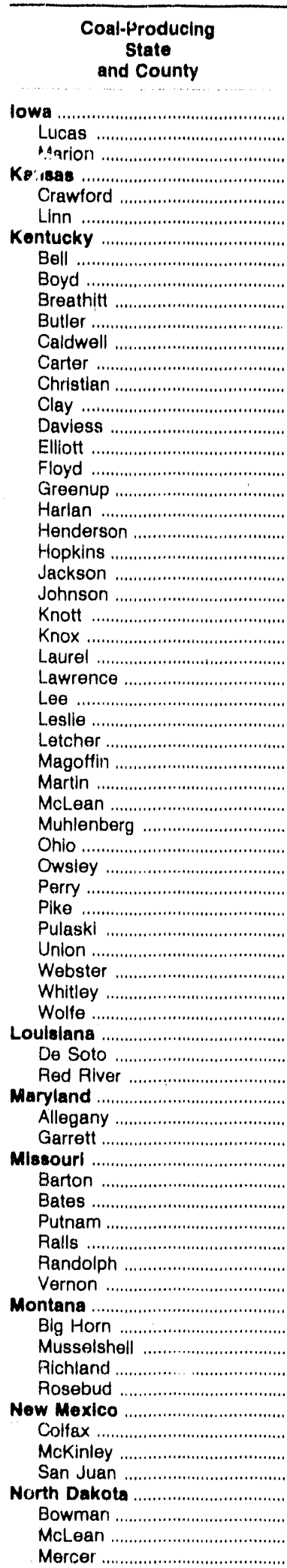

Number of Mines

Production

Average Mine Price

lowa .

\section{3}

1
2

Crawtord

Linn .......

Boll .........

Breyd ..........

Butler

Caldwell

Carter

Floyd

Greenup ،

Harian

Knot

Laurel

Letcher

Magottin

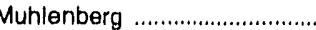

Plke

Pulaskl

Wolfe

Red River

Garrett

lesouri

Ralls

Randolph

Vernon

Blg Horn

Musselshell

Richland

Rosebud

McKinley

San Juan

Bowman ......

Mercer

See Footnotes at end of table. 

Table 23. U.S. Coal Production ${ }^{1}$, Number of Mines and Average Mine Price ${ }^{2}$
by State and County, 1989 (Continued)

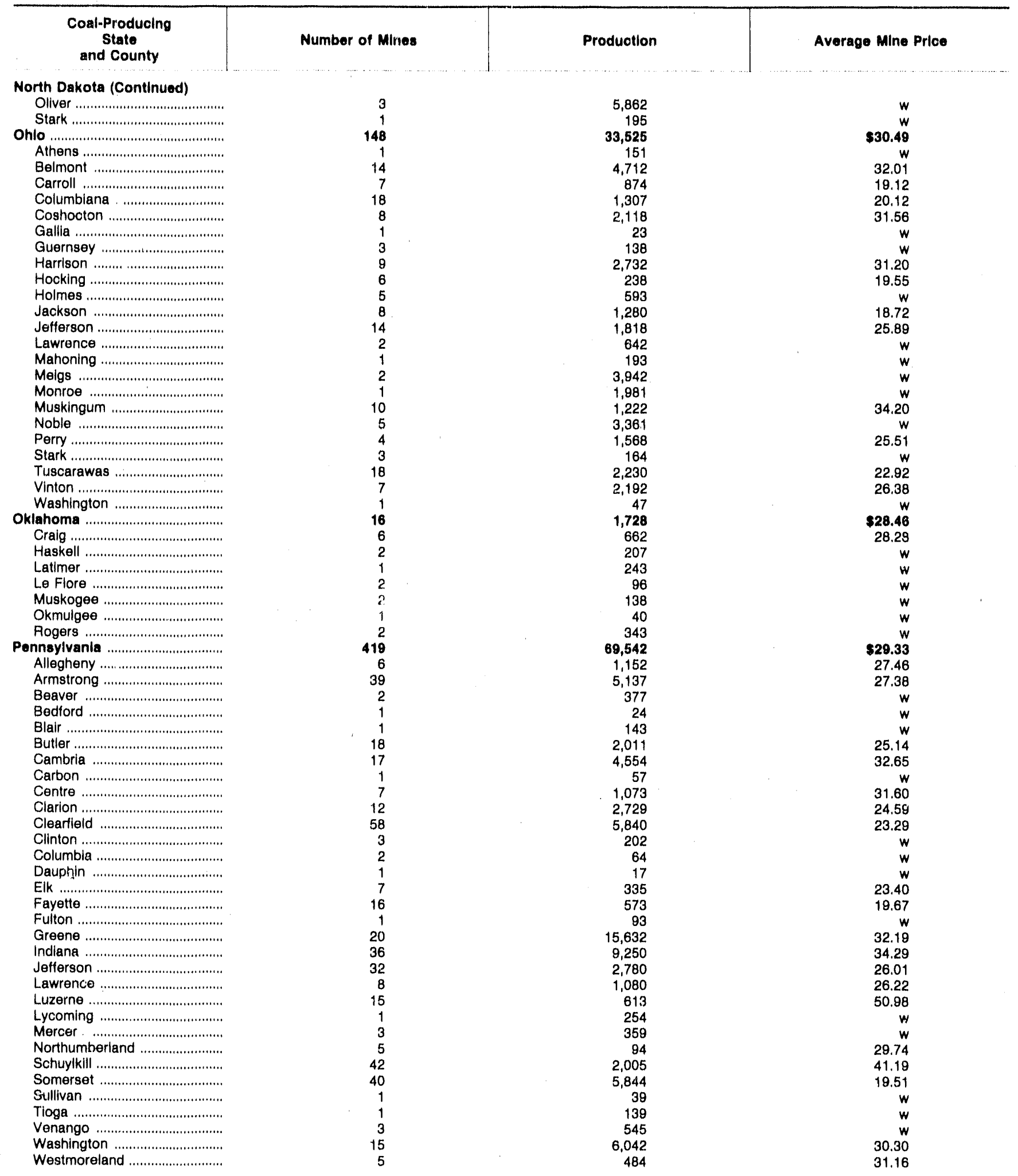

See Footnotes at end of table. 

Table 23. U.S. Coal Production ${ }^{1}$, Number of Mines and Average Mine Price
by State and County, 1989 (Continued) (Thousand Short Tons, Dollars Per Short Ton)

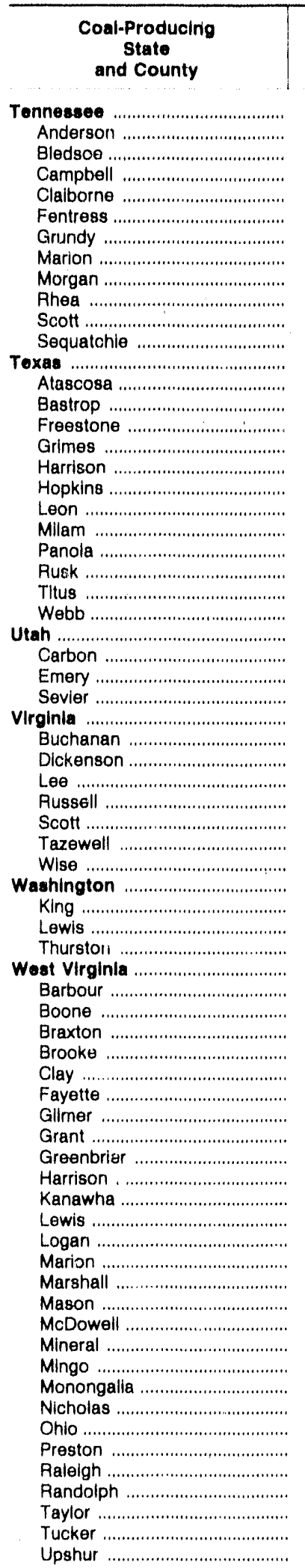

Number of Mines

Production

Average Mine Price

Anderson

8

.

6,367
1,285

147
1,445

1,445

1,600

135
55

79

186
49

253

1,133

53,848

2,984

221

5,670

3,482

3,064

2,035

8,065

5,828

8,554

3,928

9,729
287

20,099

8,177

8,563

3,059

42,766

19,005

5,819

2,479
772

41
2,723

11,928

$\mathbf{5 , 0 3 9}$

141
1,812

3,086

153,143

2,272
2,245

22,945

2,868

1,307
837

1,868

46
4,782

4,782
895

2,933

6,160

456
3,484

5,460

6,740

186
6766

446

17,745

17,162

8,698

242
4,500

4,500
7,791

1,838

64
315

1,766

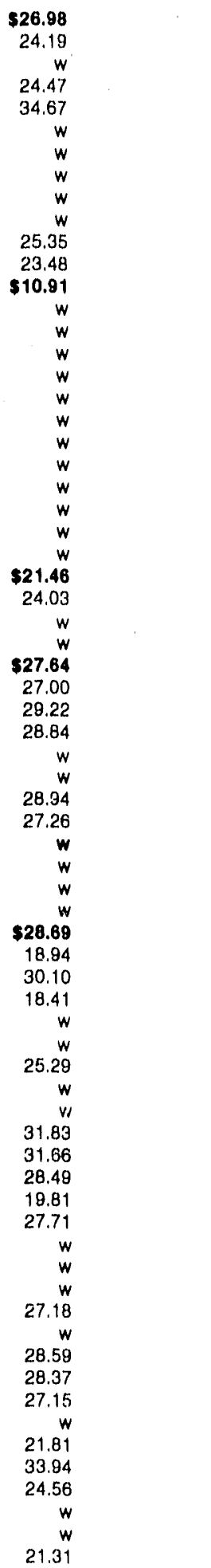

See Footnotes at end of table. 

Table 23. U.S. Coal Production ${ }^{1}$, Number of Mines and Average Niine Price ${ }^{2}$
by State and County, 1989 (Continued)

(Thousand Short Tons, Dollars Per Short Ton)

\begin{tabular}{|c|c|c|c|c|}
\hline $\begin{array}{l}\text { Coal-Producing } \\
\text { State } \\
\text { and County }\end{array}$ & Number of Mines & Production & Average Mine Prlce & \\
\hline \multicolumn{5}{|l|}{ Weat Virginia (ContInued) } \\
\hline 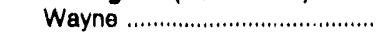 & 3 & 809 & w & \\
\hline 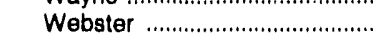 & 16 & 2,675 & 22.34 & \\
\hline 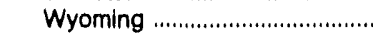 & 32 & 9,077 & 30.23 & \\
\hline 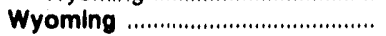 & 28 & 171,558 & $\$ 8.63$ & \\
\hline 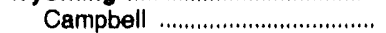 & 14 & 143,865 & 6.92 & \\
\hline 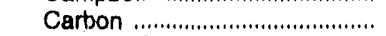 & 4 & 4,794 & w & . \\
\hline Converse ...................................... & 2 & 6,100 & $w$ & \\
\hline Hot Springs ................................. & 1 & 52 & $w$ & \\
\hline 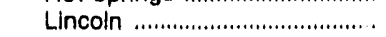 & 2. & 4,776 & $w$ & \\
\hline 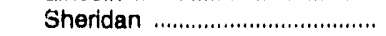 & 1 & 111 & $w$ & \\
\hline 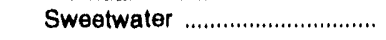 & 4 & 11,860 & $w$ & \\
\hline 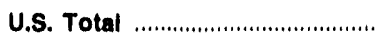 & 2,821 & 977,547 & $\$ 21.82$ & \\
\hline
\end{tabular}

1 Excludes sili, culm, refuse bank, slurry dam, and dredge production except for Pennsyivania anthracite. Excludes mines producing less than 10,000 short tons of coal during the year.

2 Average Mine Price is calculated by dividing the total free on board (t.o.b.) mine value of the coal produced by the total produotion.

w Withheld to avoid disclosure of individual company data.

Note: Total may not equal sum of components because of independent rounding.

Source: Energy Information Administration, Form EIA-7A, "Coal Production Report." 
Table 24. U.S. Coal Production ${ }^{1}$ and Average Mine Price $^{2}$ by State and Coal Rank, 1989 (Thousand Short Tons, Dollars Per Short Ton)

\begin{tabular}{|c|c|c|c|c|c|c|c|c|c|c|}
\hline \multirow{3}{*}{$\begin{array}{l}\text { Coal-Producing } \\
\text { State }\end{array}$} & \multicolumn{8}{|c|}{ Coal Rank } & \multicolumn{2}{|c|}{ Total } \\
\hline & \multicolumn{2}{|c|}{ Bltuminous } & \multicolumn{2}{|c|}{ Subbltuminous } & \multicolumn{2}{|c|}{ Lignite } & \multicolumn{2}{|c|}{ Anthracite } & \multirow[b]{2}{*}{ Production } & \multirow{2}{*}{$\begin{array}{c}\text { Average } \\
\text { Mline } \\
\text { Price }\end{array}$} \\
\hline & Production & $\begin{array}{l}\text { Average } \\
\text { Mlne } \\
\text { Price }\end{array}$ & Production & $\begin{array}{l}\text { Average } \\
\text { Mlne } \\
\text { Price }\end{array}$ & Production & $\begin{array}{l}\text { Avernge } \\
\text { Wine } \\
\text { Prics }\end{array}$ & Production & $\begin{array}{l}\text { Averikge } \\
\text { Mine } \\
\text { Price }\end{array}$ & & \\
\hline Alabama & 27,888 & 41.18 & - & - & - & - & - & - & 27,888 & 41.18 \\
\hline Alaska & - & - & 1,582 & $w$ & - & - & - & - & 1,582 & w \\
\hline 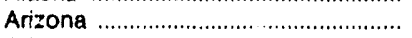 & 11,935 & $w$ & - & - & - & - & - & - & 11,935 & $w$ \\
\hline 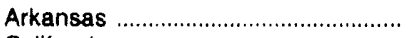 & 39 & $w$ & - & - & - & - & - & - & 39 & $w$ \\
\hline 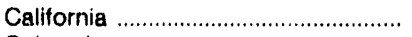 & - & - & - & - & 41 & $w$ & - & - & 41 & $w$ \\
\hline 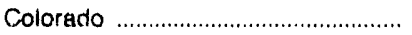 & 10,592 & $w$ & 6,522 & $w$ & - & - & - & - & 17,115 & 23.64 \\
\hline 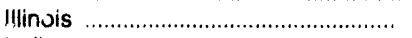 & 58,246 & 28.17 & - & - & - & - & - & - & 59,246 & 28.17 \\
\hline 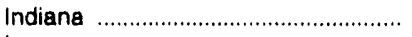 & 33,631 & 23.55 & - & - & - & - & - & - & 33,631 & 23.55 \\
\hline 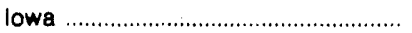 & 427 & $w$ & - & - & - & - & - & - & 427 & $w$ \\
\hline 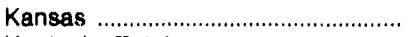 & 854 & 27.00 & - & - & - & - & - & - & 854 & 27.00 \\
\hline Kentucky Total ....................................... & 166,472 & 24.97 & - & - & - & - & .. & - & 166,472 & 24.97 \\
\hline 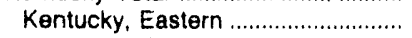 & 124,895 & 25.80 & - & - & - & - & - & - & 124,895 & 25.80 \\
\hline Kentucky, Western ....................... & 41.577 & 22.48 & - & - & - & - & - & - & 41,577 & 22.48 \\
\hline 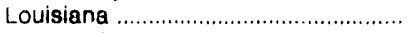 & - & - & - & - & 2,983 & $w$ & - & - & 2,983 & w \\
\hline 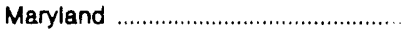 & 3,343 & 24.73 & - & - & - & - & - & - & 3,343 & 24.73 \\
\hline 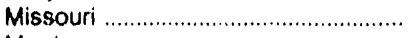 & 3,377 & $w$ & - & - & - & - & - & - & 3,377 & $w$ \\
\hline 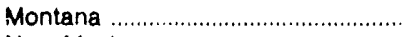 & - & - & 37,454 & $w$ & 288 & $w$ & - & - & 37,742 & 10.27 \\
\hline 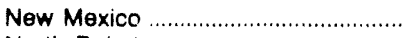 & 12,480 & $w$ & 11,216 & $w$ & - & - & - & - & 23,702 & 23.42 \\
\hline 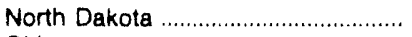 & - & - & - & - & 29,555 & 7.36 & - & - & 29,555 & 7.36 \\
\hline Ohio & 33,525 & 30.49 & - & - & - & - & - & - & 33,525 & 30.49 \\
\hline 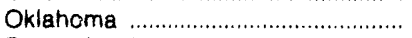 & 1,728 & 28.45 & - & - & - & - & - & - & 1,728 & 28.46 \\
\hline 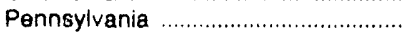 & 66,653 & 28.74 & - & - & - & - & 2,889 & 42.93 & 69,542 & 29.33 \\
\hline 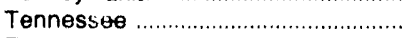 & 6,367 & 26.98 & - & - & - & .. & - & - & 6,367 & 26.98 \\
\hline 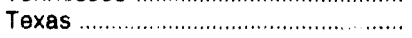 & 287 & $w$ & - & - & 53,562 & $w$ & - & - & 53,849 & 10.91 \\
\hline Utah & 20,099 & 21.46 & - & - & - & - & - & - & 20,099 & 21.46 \\
\hline Virginis & 42,766 & 27.64 & - & - & - & - & - & - & 42,766 & 27.64 \\
\hline 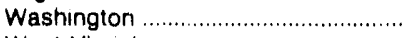 & 141 & $w$ & 4,898 & $w$ & - & - & - & - & 5,039 & $w$ \\
\hline 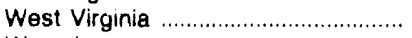 & 153,143 & 28.69 & - & - & - & - & - & - & 153,143 & 28.69 \\
\hline 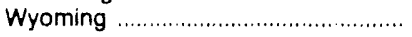 & 2.060 & $w$ & 169,498 & $w$ & - & - & - & - & 171,558 & 8.63 \\
\hline 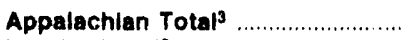 & 458,580 & $\$ 28.65$ & - & - & - & - & 2,889 & $\$ 12.93$ & 461,469 & $\$ 28.74$ \\
\hline $\begin{array}{l}\text { Interior Total } \\
\text { Western Total }\end{array}$ & $\begin{array}{r}141,165 \\
57,313\end{array}$ & $\begin{array}{l}25.34 \\
22.51\end{array}$ & $231,17 \overline{1}$ & $\$ 10.16$ & $\begin{array}{l}56,545 \\
28,884\end{array}$ & $\begin{array}{r}\$ 11.24 \\
7.40\end{array}$ & - & - & $\begin{array}{l}197,710 \\
318,368\end{array}$ & $\begin{array}{l}21.31 \\
12.12\end{array}$ \\
\hline $\begin{array}{l}\text { East of the Miss. River } \\
\text { West of the Mlses. River } \ldots \ldots \ldots \ldots \ldots \ldots \ldots \ldots\end{array}$ & $\begin{array}{r}593,033 \\
64,025\end{array}$ & $\begin{array}{l}27.88 \\
22.89\end{array}$ & $231,171^{-}$ & 10.16 & 86,429 & $9.8 \overline{1}$ & 2,889 & $\begin{array}{r}42.93 \\
-\end{array}$ & $\begin{array}{l}595,922 \\
381,625\end{array}$ & $\begin{array}{l}27.95 \\
12.25\end{array}$ \\
\hline 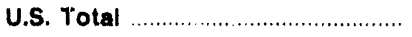 & 657,059 & 27.40 & 231,171 & 10.16 & 86,429 & 9.81 & 2,889 & 42.93 & 077,547 & 21.82 \\
\hline
\end{tabular}

1 Excludes silt, cuim, refuse bank, slurry cam, and dredge production except for Pennsylvania anthracite. Excludes mines producing less than 10,000 short tons of coal during tr.s year.

2 Average Mine Price is calculated by dividing the lotal free on board (f.o.b.) mine value of the coal produced by the total production.

3 For a detinition of coal-producing regions, see Appendix $C$

- Withheld to avoid disclosure of individual company data.

Note: Total may not equal sum of components because of independent rounding.

Source: Energy Information Administration, Form ElA-7A. "Coal Production Report." 
Table 25. Average Mine Price ${ }^{1}$ of U.S. Coal by State and Type of Mining, 1988, 1989

(Dollars Per Short Ton)

\begin{tabular}{|c|c|c|c|c|c|c|c|c|c|}
\hline \multirow{4}{*}{$\begin{array}{l}\text { Coal-Producino } \\
\text { State }\end{array}$} & \multicolumn{6}{|c|}{1989} & \multicolumn{3}{|c|}{1988} \\
\hline & \multicolumn{4}{|c|}{ Type of Mining } & \multicolumn{2}{|c|}{ Total } & \multicolumn{3}{|c|}{ Average Mine Price } \\
\hline & \multicolumn{2}{|c|}{ Underground } & \multicolumn{2}{|c|}{ Surfuce } & \multirow[b]{2}{*}{$\begin{array}{l}\text { Average } \\
\text { Mine } \\
\text { Price }\end{array}$} & \multirow[b]{2}{*}{$\begin{array}{c}\text { Percent } \\
\text { Change } \\
\text { from } \\
\text { Provious } \\
\text { Year }\end{array}$} & \multicolumn{2}{|c|}{ Type of Mining } & \multirow[b]{2}{*}{ Total } \\
\hline & $\begin{array}{l}\text { Average } \\
\text { Mine } \\
\text { Price }\end{array}$ & $\begin{array}{c}\text { Percent } \\
\text { Change } \\
\text { from } \\
\text { Prevlous } \\
\text { Yaar }\end{array}$ & $\begin{array}{l}\text { Average } \\
\text { Mine } \\
\text { Price }\end{array}$ & $\begin{array}{c}\text { Percent } \\
\text { Change } \\
\text { from } \\
\text { Provlous } \\
\text { Year }{ }^{2}\end{array}$ & & & Underground & Surface & \\
\hline Alabama & 38.96 & -2.33 & 44.31 & 3.26 & 41.18 & -0.08 & 39.89 & 42.91 & 41.22 \\
\hline Alaska & - & - & $w$ & $w$ & w & w & - & w & w \\
\hline Arizona & - & - & w & $w$ & $\mathbf{w}$ & w & - & $w$ & $w$ \\
\hline Arkansas & - & - & w & $w$ & $\mathbf{w}$ & w & - & w & $w$ \\
\hline 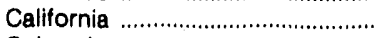 & - & - & w & $w$ & w & $w$ & - & w & $w$ \\
\hline 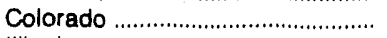 & 26.02 & -1.38 & 21.29 & 3.40 & 23.64 & 2.39 & 26.39 & 20.59 & 23.09 \\
\hline Illinois & 28.66 & -3.23 & 27.20 & 2.66 & 28.17 & -1.32 & 29.62 & 26.50 & 28.55 \\
\hline Indiana & 27.22 & -8.99 & 23.26 & -2.38 & 23.55 & -3.05 & 29.90 & 23.83 & 24.29 \\
\hline lowa & w & - & w & w & w & w & - & $w$ & $w$ \\
\hline 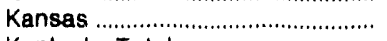 & - & - & 27.00 & -11.76 & 27.00 & -11.76 & - & 30.59 & 30.59 \\
\hline 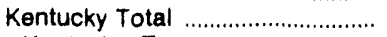 & 25.06 & -6.97 & 24.84 & -1.00 & 24.97 & -4.70 & 26.94 & 25.09 & 26.20 \\
\hline Kentucky, Eastern .............................. & 25.69 & -7.30 & 25.96 & .13 & 25.80 & -4.36 & 27.72 & 25.92 & 26.97 \\
\hline Kentucky, Western ............................. & 23.03 & -7.48 & 21.79 & -2.39 & 22.48 & -6.18 & 24.89 & 22.32 & 23.96 \\
\hline 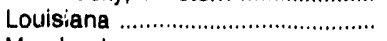 & - & - & w & $w$ & w & w & - & $w$ & w \\
\hline 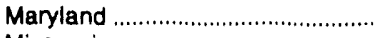 & w & $w$ & w & $w$ & 24.73 & -3.35 & w & w & 25.59 \\
\hline 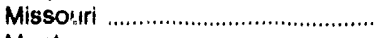 & - & - & $w$ & $w$ & w & $w$ & - & 27.06 & 27.06 \\
\hline Montana & - & - & 10.27 & 2.10 & 10.27 & 2.10 & - & 10.06 & 10.00 \\
\hline 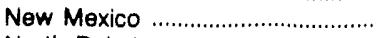 & w & $w$ & w & $w$ & 23.42 & 2.80 & $w$ & w & 22.78 \\
\hline North Dakota & - & - & 7.36 & -.23 & 7.36 & -.23 & - & 7.38 & 7.38 \\
\hline Onio & 37.30 & 1.78 & 27.24 & -.70 & 30.49 & -.04 & 36.64 & 27.44 & 30.50 \\
\hline 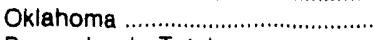 & $w$ & - & w & w & 28.46 & -6.37 & - & 31.06 & 31.06 \\
\hline 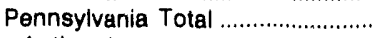 & 31.75 & $-2,94$ & 26.19 & -.45 & 29.33 & -1.92 & 32.71 & 26.31 & 28.80 \\
\hline Anthracite & 37.13 & -5.30 & 43.50 & -2.87 & 42.93 & -2.80 & 39.21 & 44.78 & 44.16 \\
\hline 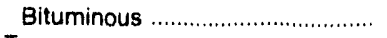 & 31.71 & -2.88 & 24.54 & .28 & 28.74 & -1.70 & 32.65 & 24.47 & 29.24 \\
\hline 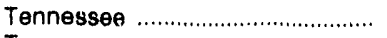 & $w$ & w & $w$ & w & 26.98 & .75 & $w$ & $w$ & 26.78 \\
\hline Taxas & - & - & 10.91 & -3.85 & 10.91 & -3.85 & - & 11.34 & 11.34 \\
\hline Utah & 21.46 & -4.27 & - & - & 21.46 & -4.27 & 22.42 & - & 22.42 \\
\hline Virginia & 28.31 & 5.27 & 24.11 & -2.02 & 27.64 & 4.32 & 26.89 & 24.60 & 26.49 \\
\hline 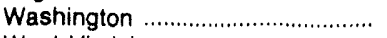 & & - & w & w & $w$ & $w$ & - & $w$ & $w$ \\
\hline West Virginia & 29.49 & 1.58 & 26.42 & 3.08 & 28.69 & 1.67 & 29.03 & 25.63 & 28.22 \\
\hline 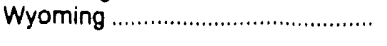 & $w$ & w & $w$ & $w$ & 8.63 & -5.74 & $w$ & $w$ & 9.16 \\
\hline Appalachlan Total ${ }^{3} \ldots \ldots \ldots \ldots \ldots$ & $\$ 22.45$ & $w$ & $\$ 27.45$ & $w$ & $\$ 28.74$ & -.50 & $\mathbf{w}$ & $\mathbf{w}$ & $\$ 28.88$ \\
\hline $\begin{array}{l}\text { Interior Total3 } \\
\text { Western Total }\end{array}$ & $\begin{array}{l}26.58 \\
22.55\end{array}$ & -3.13 & $\begin{array}{l}18.71 \\
11.03\end{array}$ & $\begin{array}{r}w \\
-1.50\end{array}$ & $\begin{array}{l}21.31 \\
12.12\end{array}$ & $\begin{array}{r}-3.27 \\
-.87\end{array}$ & $\begin{array}{r}w \\
\$ 23.27\end{array}$ & $\begin{array}{r}w \\
\$ 11.19\end{array}$ & $\begin{array}{l}22.03 \\
12.23\end{array}$ \\
\hline East of the Mlse. River & 28.94 & -1.55 & 26.43 & & & & 29.39 & & 28.26 \\
\hline West of the Mles. River .............. & 22.54 & -3.14 & 11.37 & -2.27 & 12.25 & -1.63 & 23.27 & 11.63 & 12.46 \\
\hline U.S. Total . & 28.44 & -1.82 & 17.38 & -.28 & 21.82 & -1.12 & 28.97 & 17.43 & 22.07 \\
\hline
\end{tabular}

1 Average Mine Price is calculated by dividing the totel tree on board (f.o.b.) mine value of the coal produced by the total production. Production excludes silt, culm, refuse bank, siurny dam, and dredge proctuction except for Pennsylvania anthracite. Excludes mines producing less than 10,000 short tons of coal during the year.

2 Percent changes are calculated using unrounded data.

3 For a definition of coal-producing regions, see Appendix C.

- Withneld to avoid disclosure of individual company date

Source: Energy !nformation Administration, Form ElA-7f, "Coal Production Report." 


\section{Table 26. Average Mine Price ${ }^{1}$ of U.S. Coal by District and Type of Mining, 1988, 1989 (Dollars Per Short Ton)}

\begin{tabular}{|c|c|c|c|c|c|c|c|c|c|c|}
\hline \multirow{4}{*}{\multicolumn{2}{|c|}{$\begin{array}{l}\text { Coal-Producing } \\
\text { Distrlet }\end{array}$}} & \multicolumn{6}{|c|}{1989} & \multicolumn{3}{|c|}{1988} \\
\hline & & \multicolumn{4}{|c|}{ Type of Mining } & \multicolumn{2}{|c|}{ Total } & \multicolumn{3}{|c|}{ Average Mine Price } \\
\hline & & \multicolumn{2}{|c|}{ Underground } & \multicolumn{2}{|c|}{ Surtace } & \multirow[b]{2}{*}{$\begin{array}{l}\text { Average } \\
\text { Mine } \\
\text { Price }\end{array}$} & \multirow[b]{2}{*}{$\begin{array}{c}\text { Percent } \\
\text { Change } \\
\text { from } \\
\text { Prevlous } \\
\text { Year }\end{array}$} & \multicolumn{2}{|c|}{ Type of Mining } & \multirow[b]{2}{*}{ Total } \\
\hline & & $\begin{array}{c}\text { Average } \\
\text { Mine } \\
\text { Price }\end{array}$ & $\begin{array}{c}\text { Percent } \\
\text { Change } \\
\text { from } \\
\text { Provious } \\
\text { Year' }^{2}\end{array}$ & $\begin{array}{c}\text { Average } \\
\text { Mine } \\
\text { Price }\end{array}$ & $\begin{array}{c}\text { Percent } \\
\text { Change } \\
\text { from } \\
\text { Provlous } \\
\text { Year }^{2}\end{array}$ & & & Underground & Surface & \\
\hline District & 1 & $w$ & $w$ & w & $w$ & 27.66 & -1.58 & $w$ & $w$ & 28.10 \\
\hline District & 2 & 31.63 & -2.08 & 26.51 & 1.56 & 30.56 & -.73 & 32.30 & 26.11 & 30.79 \\
\hline District & 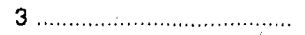 & 27.44 & -1.13 & 21.54 & 1.66 & 26.38 & -1.24 & 27.76 & 21.19 & 26.71 \\
\hline District & 4 & 37.30 & 1.78 & 27.24 & -.70 & 30.49 & -.04 & 36.64 & 27.44 & 30.50 \\
\hline District & 6 & $w$ & $w$ & $w$ & $w$ & $w$ & $w$ & w & $w$ & w \\
\hline District & 7 & 30.06 & 6.20 & 29.96 & -1.40 & 30.06 & 5.95 & 28.30 & 30.39 & 28.37 \\
\hline District & $8 \ldots$ & 27.53 & -1.64 & 26.37 & 1.39 & 27.13 & -.66 & 27.89 & 26.01 & 27.31 \\
\hline District & 9 & 23.03 & -7.48 & 21.79 & -2.39 & 22.48 & -6.18 & 24.89 & 22.32 & 23.96 \\
\hline District & 10 & 28.66 & -3.23 & 27.20 & 2.66 & 28.17 & -1.32 & 29.62 & 26.50 & 28.55 \\
\hline District & 11 & 27.22 & -8.99 & 23.26 & -2.38 & 23.55 & -3.05 & 29.90 & 23.83 & 24.29 \\
\hline District & 12 & $w$ & - & $w$ & $w$ & $w$ & $w$ & - & $w$ & $w$ \\
\hline District & $13 \ldots \ldots$ & 38.22 & -1.69 & 43.22 & 2.77 & 40.32 & 12 & 38.88 & 42.06 & 40.27 \\
\hline District & 14 & - & - & $w$ & $w$ & $w$ & $w$ & - & 24.38 & 24.38 \\
\hline District & 15 & $w$ & - & $w$ & $w$ & 12.80 & -4.48 & - & 13.40 & 13.40 \\
\hline District & 16 & - & - & $w$ & $w$ & $w$ & $w$ & - & $w$ & $w$ \\
\hline District & 17 & 26.02 & -1.97 & 21.59 & .77 & 23.74 & .61 & 26.55 & 21.43 & 23.60 \\
\hline District & 18 & - & - & 21.82 & 6.32 & 21.82 & 6.32 & - & 20.53 & 20.53 \\
\hline District & 19 & $w$ & $w$ & $w$ & $w$ & 8.63 & -5.74 & $w$ & $w$ & 9.16 \\
\hline Oistrict & 20 & 21.46 & -4.27 & - & - & 21.46 & -4.27 & 22.42 & - & 22.42 \\
\hline District & $21 \ldots$ & - & - & 7.36 & -.23 & 7.36 & -.23 & - & 7.38 & 7.38 \\
\hline District & 22 & - & - & 10.27 & 2.10 & 10.27 & 2.10 & - & 10.06 & 10.06 \\
\hline District & 23 & - & - & $w$ & $w$ & $w$ & $w$ & - & $w$ & $w$ \\
\hline District & $24^{3}$ & 37.13 & -5.30 & 43.50 & -2.87 & 42.93 & -2.80 & 39.21 & 44.78 & 44.16 \\
\hline District & t 1-233 Total .................... & $\$ 28.44$ & -1.81 & $\$ 17.27$ & -.19 & $\$ 21.76$ & -1.07 & $\$ 28.26$ & $\$ 17.30$ & $\$ 22.00$ \\
\hline U.S. To & & 28.44 & -1.82 & 17.38 & -.28 & 29.82 & -1.12 & 28.97 & 17.43 & 22.07 \\
\hline
\end{tabular}

1 Average Mine Price is calculated by dwiding the lotal free on board (f.o.b.) mine value of the coal produced by the total production. Production ex cludes sil, culm, refuse bank, slurry dam, and dredge production except for Pennsylvania anthracite. Excludes mines producing less than 10,000 short tons of coal duing the year.

2 Percerit changes are calculated using unrounded data.

3 District 24 is the anthracite-producing district in Pennsytvania. Distncts 1.23 represent tho tota! U.S. pruduction of bituminous, subbituminous coal, and lignite. See Appendix

- Withheld to avord disclosure of indwidual company data

Source: Energy Information Administration, Form ElA.7A, "Coal Production Report." 


\section{Table 27. Average Mine Price ${ }^{1}$ of U.S. Coal by Mine Production Range and Type of Mining, 1988, 1989}

(Dollars Per Short Ton)

\begin{tabular}{|c|c|c|c|c|c|c|c|c|c|}
\hline \multirow{4}{*}{$\begin{array}{l}\text { Mine Production Range } \\
\text { (Thousand Short Tons) }\end{array}$} & \multicolumn{6}{|c|}{1989} & \multicolumn{3}{|c|}{1988} \\
\hline & \multicolumn{4}{|c|}{ Type of Wining } & \multicolumn{2}{|c|}{ Total } & \multicolumn{3}{|c|}{ Average Mine Price } \\
\hline & \multicolumn{2}{|c|}{ Underground } & \multicolumn{2}{|c|}{ Surface } & \multirow[b]{2}{*}{$\begin{array}{c}\text { Average } \\
\text { Mine } \\
\text { Price }\end{array}$} & \multirow[b]{2}{*}{$\begin{array}{c}\text { Percent } \\
\text { Change } \\
\text { from } \\
\text { Prevlous } \\
\text { Yoar? }\end{array}$} & \multicolumn{2}{|c|}{ Type of Mining } & \multirow[b]{2}{*}{ Total } \\
\hline & $\begin{array}{c}\text { Average } \\
\text { Mine } \\
\text { Price }\end{array}$ & $\begin{array}{c}\text { Percent } \\
\text { Change } \\
\text { from } \\
\text { Prevlous } \\
\text { Year }{ }^{2}\end{array}$ & $\begin{array}{c}\text { Average } \\
\text { Mine } \\
\text { Price }\end{array}$ & $\begin{array}{c}\text { Percent } \\
\text { Change } \\
\text { from } \\
\text { Prevlous } \\
\text { Year? }\end{array}$ & & & Underground & Surface & \\
\hline 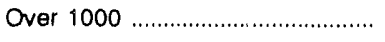 & 30.26 & -1.00 & 14.23 & 2.21 & 19.40 & 0.83 & 30.57 & 13.92 & 18.24 \\
\hline 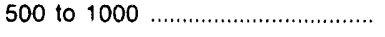 & 29.14 & -4.11 & 27.21 & -.54 & 28.27 & -2.32 & 30.38 & 27.36 & 28.94 \\
\hline 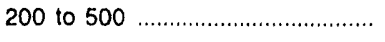 & 25.81 & -4.37 & 24.95 & -1.85 & 25.39 & -3.29 & 26.99 & 25.42 & 26.26 \\
\hline 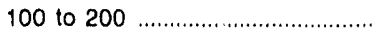 & 25.37 & -2.39 & 23.80 & -1.21 & 24.71 & -1.83 & 25.99 & 24.09 & 25.17 \\
\hline 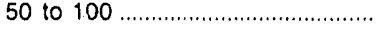 & 25.02 & .58 & 24.10 & 1.71 & 24.61 & 1.19 & 24.88 & 23.69 & 24.31 \\
\hline 10 to 50 & 25.31 & -2.20 & 24.34 & -.40 & 24.77 & -1.37 & 25.88 & 24.44 & 25.12 \\
\hline 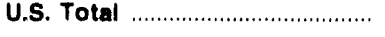 & $\$ 28.44$ & -1.82 & $\$ 17.38$ & -.28 & $\$ 21.82$ & -1.12 & $\$ 28.97$ & $\$ 17.43$ & $\$ 22.07$ \\
\hline
\end{tabular}

1 Average Mine Price is calculated by dividing the total free on board (f.o.b.) mine value of the coal produced by the total production. Production excludes silt, culm, refuse bank, slurry dam, and dredge production except for Pennsylvania anthracite. Excludes mines producing less than 10,000 short tons of coal during the year

2 Percent changes are calculated using unrounded data.

Source: Energy Information Administration, Form ElA-7A, "Coal Production Report." 


\section{Productivity and Employment}

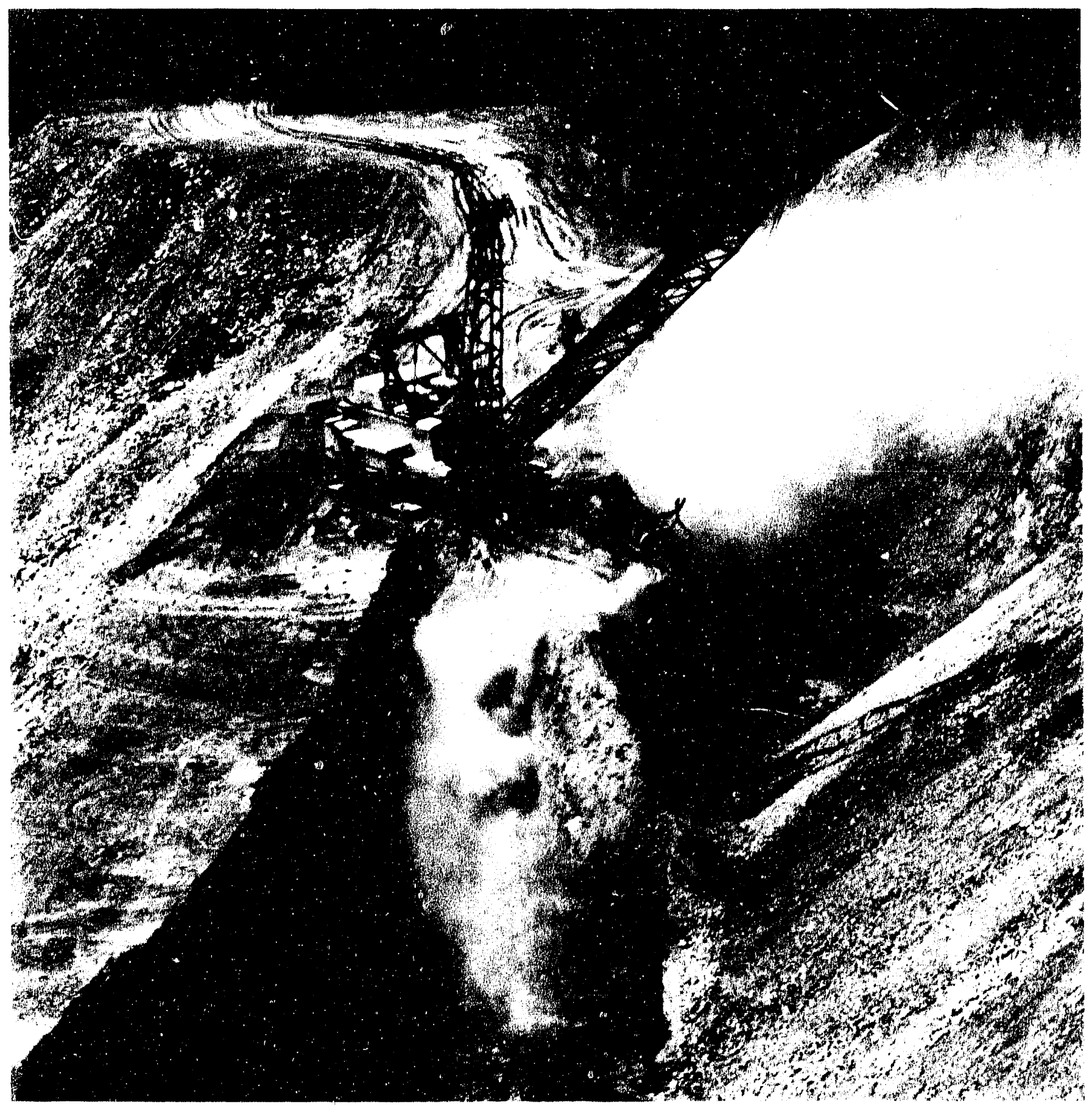

A dragline is in the process of removing overburden at Ashland Oil, Inc. 's Big John Mine. 
Figure 6. U.S. Coal Mining Productivity by Type of Mining and by Region, 1980-1989
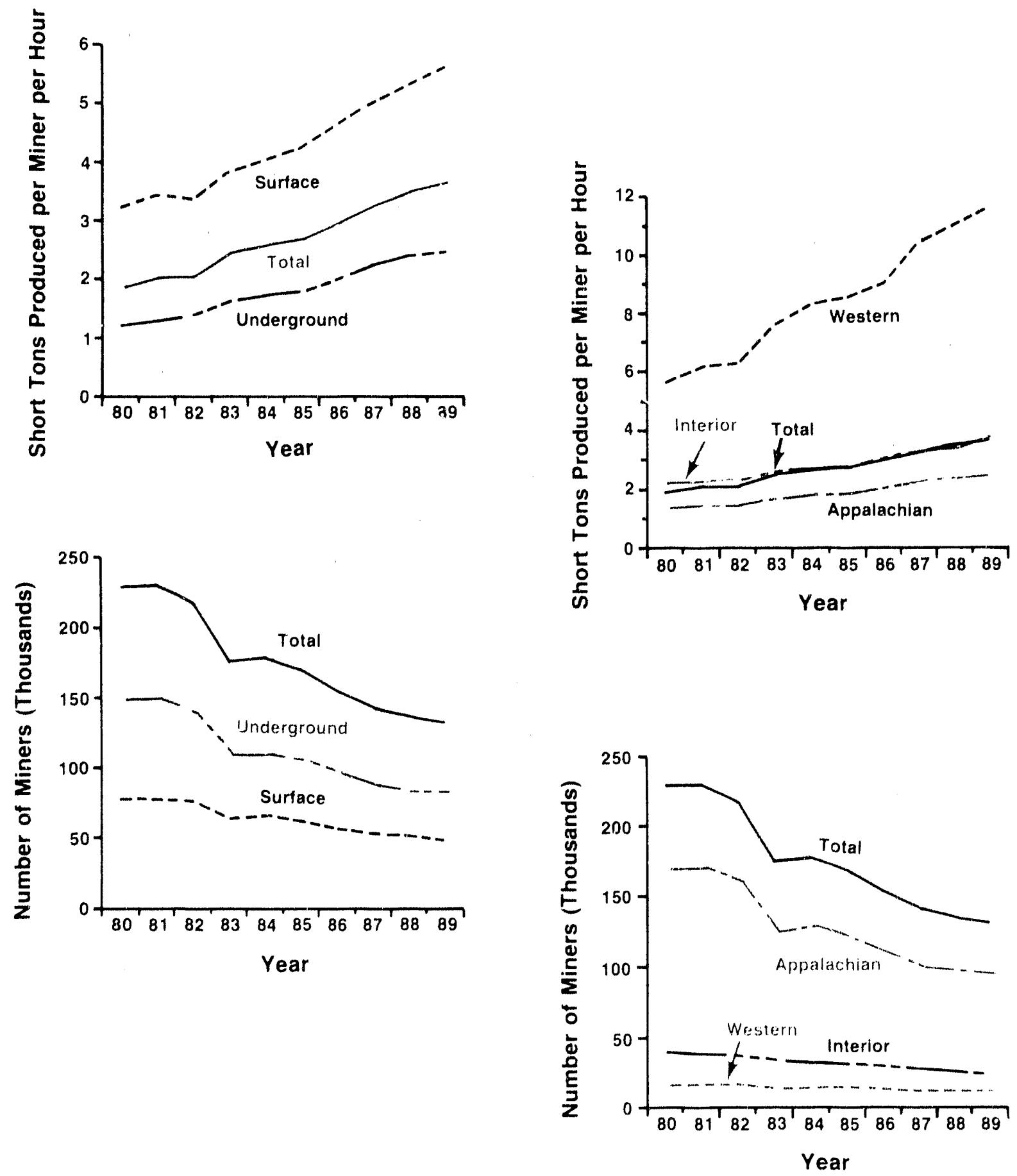

Note: Scale has been enlarged to show detail in the short tons produced per miner per hour by type of mining plot

Source: Energy Information Administration. Form ElA-7A, "Coal Production Report." 
Table 28. U.S. Coal Mining Productivity by State, 1989

\begin{tabular}{|c|c|c|c|c|c|c|}
\hline $\begin{array}{l}\text { Coal-Producing } \\
\text { State }\end{array}$ & $\begin{array}{c}\text { Number of } \\
\text { Mining } \\
\text { Operations }\end{array}$ & $\begin{array}{c}\text { Cosal } \\
\text { Production } \\
\text { (thousand } \\
\text { short tons) }\end{array}$ & $\begin{array}{l}\text { Average Number } \\
\text { of } \\
\text { Miners Working } \\
\text { Dally }\end{array}$ & $\begin{array}{l}\text { Average Number } \\
\text { of Days Worked } \\
\text { During the Year }\end{array}$ & $\begin{array}{l}\text { Average } \\
\text { Production } \\
\text { per Miner } \\
\text { per Hour' } \\
\text { (short tons) }\end{array}$ & $\begin{array}{l}\text { Average } \\
\text { Production } \\
\text { per Miner } \\
\text { per Shift } \\
\text { (short tons) }\end{array}$ \\
\hline Alabama & 86 & 27,888 & 6,505 & 207 & 2.25 & 19.44 \\
\hline 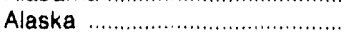 & 1 & 1,582 & 87 & 265 & 7.73 & 69.60 \\
\hline Arizona & 2 & 11,935 & 780 & 205 & 6.25 & 75.00 \\
\hline Arkansas .................................. & 1 & 39 & 8 & 300 & 1.75 & 15.73 \\
\hline 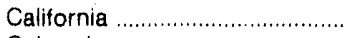 & 1 & 41 & 5 & 124 & 8.21 & 65.69 \\
\hline Colorado & 23 & 17,115 & 2,019 & 224 & $\Delta .08$ & 35.74 \\
\hline 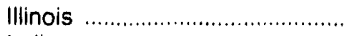 & 54 & 59,246 & 10,003 & 238 & 2.77 & 22.98 \\
\hline 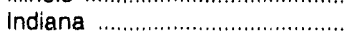 & 62 & 33,631 & 3,684 & 256 & 3.86 & 35.42 \\
\hline lowa & 3 & 427 & 149 & 210 & 151 & 14.37 \\
\hline 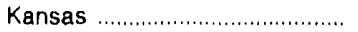 & 4 & 854 & 165 & 234 & 2.66 & 21.30 \\
\hline Kentucky Total ........................... & 1,103 & 166,472 & 30,656 & 208 & 2.78 & 23.96 \\
\hline Kentucky, Eastern .................... & 1,004 & 124,895 & 24,620 & 208 & 2.58 & 22.19 \\
\hline Kentucky, Western ................... & 99 & 41,577 & 6,036 & 203 & 3.62 & 32.03 \\
\hline Louisiana & 2 & 2,983 & 69 & 186 & 15.04 & 135.32 \\
\hline 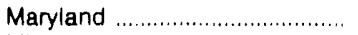 & 25 & 3,343 & 554 & 221 & 3.00 & 25.97 \\
\hline Missouri .......................................... & 8 & 3,377 & 428 & 211 & 3.43 & 26.46 \\
\hline Montana & 8 & 37,742 & 682 & 291 & 18.94 & 165.05 \\
\hline New Mexico .............................. & 8 & 23,702 & 1,470 & 223 & 7.93 & 63.45 \\
\hline North Dakota & 11 & 29,555 & 850 & 231 & 15.69 & 131.90 \\
\hline Ohio & 170 & 33,525 & 7,374 & 226 & 2.33 & 21.14 \\
\hline 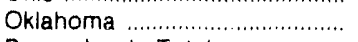 & 16 & 1,728 & 414 & 214 & 2.15 & 18.32 \\
\hline Pennsylvania Total ..................... & 496 & 69,542 & 15,469 & 234 & 2.28 & 19.48 \\
\hline 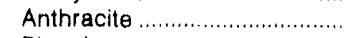 & 103 & 2,889 & 1,394 & 248 & 1.12 & 8.87 \\
\hline 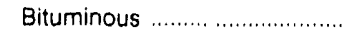 & 393 & 66,653 & 14,075 & 231 & 2.39 & 20.79 \\
\hline Tennesser ................................. & 87 & 6,367 & 1,857 & 225 & 1.72 & 14.52 \\
\hline Texas & 14 & 53,849 & 2,109 & 308 & 7.31 & 74.54 \\
\hline 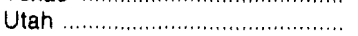 & 21 & 20,099 & 2,169 & 219 & 4.75 & 40.12 \\
\hline Virginia & 403 & 42,766 & 10,371 & 212 & 2.21 & 18.30 \\
\hline 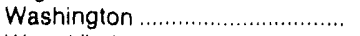 & 3 & 5,039 & 787 & 200 & 3.34 & 28.94 \\
\hline 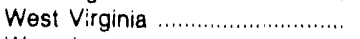 & 871 & 153,143 & 29,482 & 198 & 2.77 & 23.62 \\
\hline 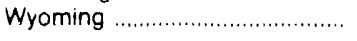 & 28 & 171,558 & 3,351 & 271 & 20.28 & 184.65 \\
\hline $\begin{array}{l}\text { Appalachian Totap } \\
\text { Interlor Tota }\end{array}$ & $\begin{array}{r}3,1,2 \\
263\end{array}$ & $\begin{array}{l}461,469 \\
197710\end{array}$ & 96,232 & 211 & 2.49 & 21.32 \\
\hline Wostern Total ${ }^{8}$ & 106 & 318,368 & 12,200 & 240 & $\begin{array}{r}5.64 \\
11.63\end{array}$ & $\begin{array}{r}33.84 \\
101.69\end{array}$ \\
\hline $\begin{array}{l}\text { East of the Miss. Rlver } \ldots \ldots \ldots . \\
\text { West of the Miss. River } \ldots \ldots \ldots .\end{array}$ & $\begin{array}{r}3,357 \\
154\end{array}$ & $\begin{array}{l}595,922 \\
381,625\end{array}$ & $\begin{array}{r}115,955 \\
15,542\end{array}$ & $\begin{array}{l}212 \\
241\end{array}$ & $\begin{array}{r}2.63 \\
10.21\end{array}$ & $\begin{array}{l}22.52 \\
89.91\end{array}$ \\
\hline U.S. Total & 3,511 & 977,547 & 131,497 & 214 & 3.70 & 31.75 \\
\hline
\end{tabular}

1 Includes preparation plants.

2 Excludes silt, culm, refuse bank, slurry dam, and dredge production except for Pennsylvania anthracite. Excludes mines producing less than 10,000 short tons of coal during the year.

3 Includes all employees engaged in production, preparation, processing, development, maintenance, repair, shop or yard work at mining operations. Exsludes office workers. Includes mining operations management and all technical and engineering personnel.

- Calculated by dividing total coal production by the total diract labor hours worked by all mine employees identified in footnote 3 above.

s Calculated by multiplying average production per miner per hour by the average length of a miner shift.

- For a definition of coal-producing regions, see Appendix C.

Note: Total may not equal sum of components because of indepenuent rounding.

Source: Energy information Administration, Form EIA.7A, "Coal Production Report." 
Table 29. U.S. Underground Coal Mining Productivity by State, 1989

\begin{tabular}{|c|c|c|c|c|c|c|}
\hline $\begin{array}{l}\text { Coal-Producing } \\
\text { State }\end{array}$ & $\begin{array}{l}\text { Number of } \\
\text { Alning } \\
\text { Operations ' } 2\end{array}$ & $\begin{array}{l}\text { Coal } \\
\text { Production² } \\
\text { (theusand } \\
\text { ahort tons) }\end{array}$ & $\begin{array}{c}\text { Average Number } \\
\text { of } \\
\text { Miners Working } \\
\text { Dally }\end{array}$ & $\begin{array}{l}\text { Average Number } \\
\text { of Days Worked } \\
\text { During the Year }\end{array}$ & $\begin{array}{l}\text { Average } \\
\text { Production } \\
\text { per Mlner } \\
\text { per Hour } \\
\text { (short tons) }\end{array}$ & $\begin{array}{l}\text { Average } \\
\text { Production } \\
\text { per Miner } \\
\text { per Shlits } \\
\text { (short tons) }\end{array}$ \\
\hline$\cdots \cdots+n^{\prime} \cdots$ & & & & & & \\
\hline 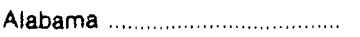 & 13 & 16,307 & 4,371 & 2.12 & 2.00 & 16.04 \\
\hline Cotorado & 16 & 8,502 & 1,490 & 233 & 2.91 & 24.50 \\
\hline 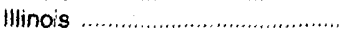 & 35 & 39,328 & 8,084 & 238 & 2.41 & 20.35 \\
\hline Indiana & 7 & 2,518 & 521 & 223 & 2.47 & 20.98 \\
\hline lowa & 1 & 36 & 15 & 121 & 2.06 & 19.58 \\
\hline Kentucky Total & 673 & 98,591 & 20,752 & 208 & 2.54 & 21.10 \\
\hline Kentucky, Eastern ....................... & 639 & 75.270 & 16,586 & 208 & 2.40 & 19.88 \\
\hline Kentucky, Western & 34 & 23,321 & 4,166 & 218 & 3.13 & 27.15 \\
\hline 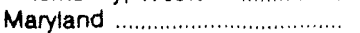 & 2 & 1,834 & 282 & 237 & 3.09 & 27.82 \\
\hline New Mexjco & 2 & 45 & 49 & 100 & 1.03 & 8.73 \\
\hline 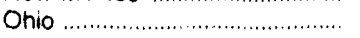 & 20 & 10,824 & 2,953 & 228 & 2.00 & 17.76 \\
\hline Oklahoma & 1 & 40 & 36 & 86 & 1.28 & 12.15 \\
\hline Pennsylvania Total ....................... & 104 & 39,326 & 9,578 & 235 & 2.16 & 17.59 \\
\hline Anthracite $\ldots \ldots \ldots \ldots \ldots \ldots \ldots$ & 20 & 259 & 193 & 256 & .71 & 5.56 \\
\hline Bituminous & 84 & 39,067 & 9,385 & 231 & 2.19 & 18.02 \\
\hline 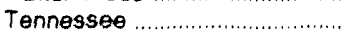 & 58 & 4,597 & 1,471 & 220 & 1.58 & 13.17 \\
\hline Utah & 21 & 20,099 & 2,169 & 219 & 4.75 & 40.12 \\
\hline Virginia & 294 & 35,919 & 8,889 & 210 & 2.15 & 17.70 \\
\hline 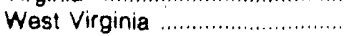 & 529 & 113,006 & 23,048 & 200 & 2.59 & $21.6 \hat{2}$ \\
\hline 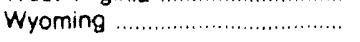 & 3 & 1,645 & 280 & 209 & 3.21 & 27.85 \\
\hline Appalachian Totap ................ & 1,650 & 297,082 & 67,178 & 208 & 2.34 & 19.40 \\
\hline Interior Totar & 78 & 65,244 & 12,822 & 224 & 2.63 & 22.52 \\
\hline 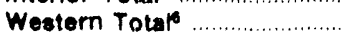 & 42 & 30,292 & 3,988 & 218 & 3.83 & 33.22 \\
\hline $\begin{array}{l}\text { East of the Miss. River ........ } \\
\text { West of the Mlas. River ....... }\end{array}$ & $\begin{array}{r}1,735 \\
44\end{array}$ & $\begin{array}{r}362,251 \\
30,367\end{array}$ & $\begin{array}{r}79,949 \\
4,039\end{array}$ & $\begin{array}{l}209 \\
213\end{array}$ & $\begin{array}{l}2.39 \\
3.92\end{array}$ & $\begin{array}{l}19.82 \\
33.28\end{array}$ \\
\hline 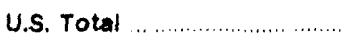 & 1,779 & 392,618 & 83,988 & 200 & 2.46 & 20.45 \\
\hline
\end{tabular}

1 Includes preparation plants.

2 Excludes silt, culm, refuse bank, slurry dam, and dradge production except for Pennsylvania anthracite. Excludes mines producing less than 10,000 short tons of coal during the year.

3 includes all employes engaged in production, preparation. processing, develupment, maintenance, repair, shop or yard work at mining operations. Ex. cludes oftice workers. Includes mining operations management and all technical and engineering personnel.

4 Calculated by dividing total coal production by the total direct labor hours worked by ail mine employees identified in footnote 3 anove.

- Calculated by multiplying average production per miner per hour by the average length of a miner shitt.

- For a definition of coal-producing regions, see Appendix C.

Note: Total may not equal sum of components because of independent rounding

Source: Energy information Administration. Form EIA-7A. "Coal Production Report." 
Table 30. U.S. Surface Coal Mining Productivity by State, 1989

\begin{tabular}{|c|c|c|c|c|c|c|}
\hline $\begin{array}{l}\text { Coal-Producing } \\
\text { State }\end{array}$ & $\begin{array}{c}\text { Number of } \\
\text { Mlning } \\
\text { Operations }\end{array}$ & $\begin{array}{l}\text { Coal } \\
\text { Production? } \\
\text { (thousand } \\
\text { short tons) }\end{array}$ & $\begin{array}{c}\text { Average Number } \\
\text { of } \\
\text { Miners Working } \\
\text { Dally }\end{array}$ & $\begin{array}{l}\text { Average Number } \\
\text { of Daye Worked } \\
\text { During ttie Year }\end{array}$ & $\begin{array}{l}\text { Average } \\
\text { Production } \\
\text { per Mlner } \\
\text { per Hour } \\
\text { (ehort tons) }\end{array}$ & $\begin{array}{l}\text { Average } \\
\text { Productlon } \\
\text { per Mlner } \\
\text { per Shltt' } \\
\text { (ehort tons) }\end{array}$ \\
\hline 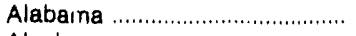 & 73 & 11,581 & 2,134 & 206 & 2.72 & 23.82 \\
\hline Alaska & 1 & 1,582 & 87 & 265 & 7.73 & 69.60 \\
\hline 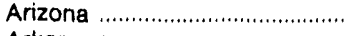 & 2 & 11,935 & 780 & 205 & 6.25 & 75.00 \\
\hline 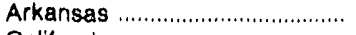 & 1 & 39 & 8 & 300 & 1.75 & 15.73 \\
\hline 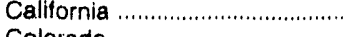 & 1 & 41 & 5 & 124 & 8.21 & 65.68 \\
\hline 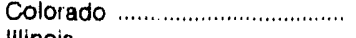 & 7 & 8,612 & 529 & 205 & 6.74 & 64.48 \\
\hline 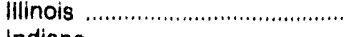 & 19 & 19,817 & 1,919 & 236 & 3.96 & 31.60 \\
\hline 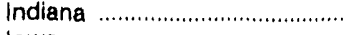 & 55 & 31,112 & 3,163 & 260 & 4.04 & 37.46 \\
\hline lowa & 2 & 391 & 134 & 255 & 1.48 & 14.03 \\
\hline 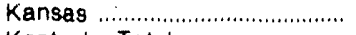 & 4 & 854 & 165 & 234 & 2.66 & 21,30 \\
\hline 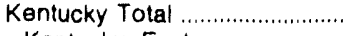 & 430 & 67,881 & 9,904 & 206 & 3.23 & 29.36 \\
\hline Kentucky, Eastern .................... & 365 & 49,625 & 8,034 & 208 & 2.92 & 26.64 \\
\hline Kentucky, Western .................. & 65 & 18,256 & 1,870 & 195 & 4.53 & 40.47 \\
\hline 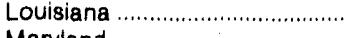 & 2 & 2,983 & 69 & 186 & 15.04 & 135.32 \\
\hline 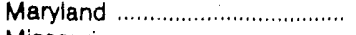 & 23 & 1,509 & 272 & 220 & 2.90 & 25.01 \\
\hline 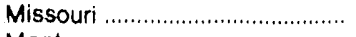 & 8 & 3,377 & 428 & 211 & 3.43 & 28.46 \\
\hline 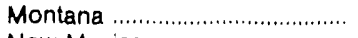 & 8 & 37,742 & 682 & 291 & 18.94 & 165.05 \\
\hline New Mexico ............................... & 6 & 23,657 & 1,421 & 264 & 8.03 & 62.83 \\
\hline 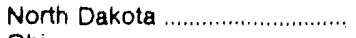 & 11 & 29,555 & 850 & 231 & 15.69 & 131.90 \\
\hline Ohio & 150 & 22,701 & 4,421 & 226 & 2.52 & 22.89 \\
\hline 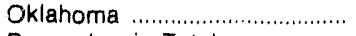 & 15 & 1,688 & 378 & 223 & 2.18 & 18.48 \\
\hline Pennsylvania Total . ................. & 392 & 30,216 & 5,881 & 234 & 2.47 & 21.34 \\
\hline 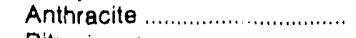 & 83 & 2,630 & 1,201 & 246 & 1.19 & 9.43 \\
\hline 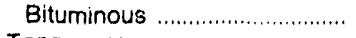 & 309 & 27,585 & 4,690 & 231 & 2.76 & 24.30 \\
\hline 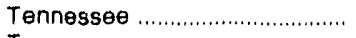 & 29 & 1,770 & 386 & 235 & 2.20 & 19.20 \\
\hline Texas & 14 & 53,849 & 2,108 & 308 & 7.31 & 74.54 \\
\hline Virginia & 109 & 6,847 & 1,482 & 219 & 2.59 & 21.77 \\
\hline 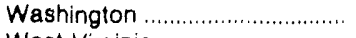 & 3 & 5,039 & 787 & 200 & 3.34 & 28.94 \\
\hline West Virginia ............................... & 342 & 40,137 & 6,434 & 195 & 3.49 & 30.55 \\
\hline 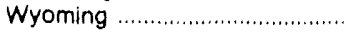 & 25 & 169,913 & 3,071 & 279 & 21.38 & 195.81 \\
\hline Appaiachian Total & 1,483 & 164,387 & 29,054 & 215 & 2.84 & 25.03 \\
\hline Interior Total & 185 & 132,466 & 10,243 & 232 & 4.87 & 44.31 \\
\hline Weatern Total ${ }^{\circ}$......................... & 64 & 288,076 & 8,212 & 254 & 14.64 & 130.80 \\
\hline East of the Miss. River ......... & 1,622 & 233,672 & 36,006 & 216 & 3.13 & 27.63 \\
\hline Weat of the Mies. Rlver ........ & 110 & 351,257 & 11,503 & 252 & 11.86 & 105.68 \\
\hline 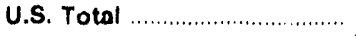 & 1,732 & 584,929 & 47,509 & 218 & 5.61 & 49.56 \\
\hline
\end{tabular}

1 Includes preparation plants.

2 Excludes silt, culm, refuse bank, slurry dam, and dredge production except for Pennsylvania anthracite. Excludes mines producing less than 10,000 short tons of coal during the year.

3 Includes all employees engaged in production, preparation, processing, development, maintenance, repair, shop or yard work at mining operations. Excludes office workers. Includes mining operations management and all technical and engineering personnel.

4 Calculated by dividing total coal production by the total direct labor hours worked by all mine employees identified in footnote 3 above.

- Calculated by multiplying average production per miner per hour by the average length of a miner shift.

- For a definition of coal-producing regions, see Appendix C.

Note: Total may not equal sum of components because of independent rounding.

Source: Energy Information Administration, Form EIA-7A, "Coal Production Report." 
Table 31. U.S. Coal Mining Productivity by District, 1989

\begin{tabular}{|c|c|c|c|c|c|c|}
\hline $\begin{array}{l}\text { Coai-Producing } \\
\text { Dlstrict }\end{array}$ & $\begin{array}{c}\text { Number of } \\
\text { Mining } \\
\text { Operations } 12\end{array}$ & $\begin{array}{c}\text { Coal } \\
\text { Production } \\
\text { (thousand } \\
\text { short tons) }\end{array}$ & $\begin{array}{c}\text { Average Number } \\
\text { of } \\
\text { Miners Working } \\
\text { Dally }\end{array}$ & $\begin{array}{l}\text { Average Number } \\
\text { of Days Worked } \\
\text { During the YGar }\end{array}$ & $\begin{array}{l}\text { Average } \\
\text { Productlon } \\
\text { per Miner } \\
\text { per Hour } \\
\text { (short tons) }\end{array}$ & $\begin{array}{l}\text { Average } \\
\text { Productlon } \\
\text { per Miner } \\
\text { per Shltt' } \\
\text { (short tone) }\end{array}$ \\
\hline 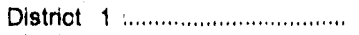 & 324 & 45,739 & 10,146 & 230 & 2.28 & 19.91 \\
\hline 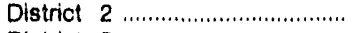 & 110 & 29,799 & 5,618 & 229 & 2.65 & 22.64 \\
\hline 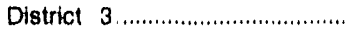 & 243 & 42,492 & 7,701 & 209 & 2.74 & 23.56 \\
\hline 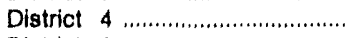 & 170 & 33,525 & 7,374 & 226 & 2.33 & 21.14 \\
\hline District $6 \ldots \ldots \ldots \ldots \ldots \ldots \ldots \ldots \ldots \ldots$ & 10 & 8,289 & 1,539 & 244 & 2.47 & 20.90 \\
\hline District $7 \ldots \ldots \ldots \ldots \ldots \ldots \ldots \ldots \ldots \ldots \ldots$ & 133 & 15,462 & 3,787 & 193 & 2.29 & 19.23 \\
\hline District 8 & 1,836 & 253,923 & 51,786 & 206 & 2.59 & 22.05 \\
\hline 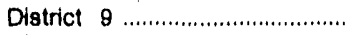 & 99 & 41,577 & 6,036 & 203 & 3.62 & 32.03 \\
\hline 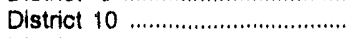 & 54 & 59,246 & 10,003 & 238 & 2.77 & 22.98 \\
\hline 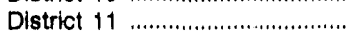 & 62 & 33,631 & 3,684 & 256 & 3.86 & 35.42 \\
\hline 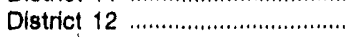 & 3 & 427 & 149 & 210 & 1.51 & 14.37 \\
\hline 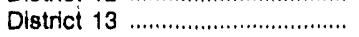 & 113 & 29,351 & 6,887 & 210 & 2.24 & 18.14 \\
\hline District 14 & 5 & 342 & 89 & 277 & 1.69 & 14.23 \\
\hline District 15 & 40 & 62,488 & 3,104 & 242 & 6.58 & 58.90 \\
\hline District $16 \ldots \ldots \ldots \ldots \ldots \ldots$ & 1 & 27 & 8 & 192 & 1.80 & 16.21 \\
\hline 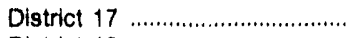 & 25 & 17,588 & 2,150 & 213 & 4.00 & 34.85 \\
\hline 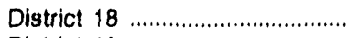 & 8 & 35,178 & 2,116 & 246 & 7.48 & 66.52 \\
\hline 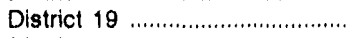 & 28 & 171,558 & 3,351 & 271 & 20.28 & 184.65 \\
\hline 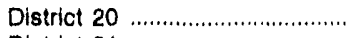 & 21 & 20,099 & 2,169 & 210 & 4.75 & 40.12 \\
\hline 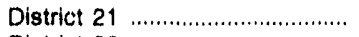 & 11 & 29,555 & 850 & 231 & 15.69 & 131.90 \\
\hline District $22 \ldots \ldots \ldots \ldots \ldots \ldots \ldots \ldots \ldots$ & 8 & 37,742 & 682 & 291 & 18.84 & 165.05 \\
\hline 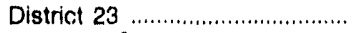 & 4 & 6,621 & 874 & 216 & 3.86 & 33.81 \\
\hline 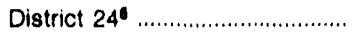 & 103 & 2,889 & 1,394 & 248 & 1.12 & 8.87 \\
\hline 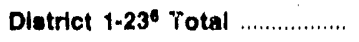 & 3,408 & 974,658 & 130,103 & 213 & 3.73 & 32.05 \\
\hline 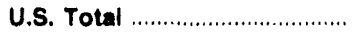 & 3,511 & 977,547 & 131,497 & 214 & 3.70 & 31.75 \\
\hline
\end{tabular}

1 Includes preparation plants.

2 Excludes silt, culm, refuse bank, slurry dam, and dredge production excepl for Pennsylvania anthracite. Excludes mines producing less than 10,000 short tons of coal during the year.

3 Includes all employees engaged in production, preparation, processing, development, maintenance, repair, shop or yard work at mining operations. Excludes office workers. Includes mining operations management and all technical and engineering personnel.

4 Calculated by dividing total coal production by the total direct labor hours worked by all mine employees identified in footriote 3 above.

- Calculated by multiplying average production per ininer per hour by the average length of a miner shift.

- District 24 is the anthracite-pindiscing district in Pennsylvania. Districts 1-23 represent the total U.S. production of bituminous, subbituminous coal, and lignite. See Appendix C

Note: Total may not equal sum of components because of independent rounding.

Source: Energy Information Administration, Form EIA-7A, "Coal Production Report." 
Table 32. U.S. Underground Coal Mining Productivity by District, 1989

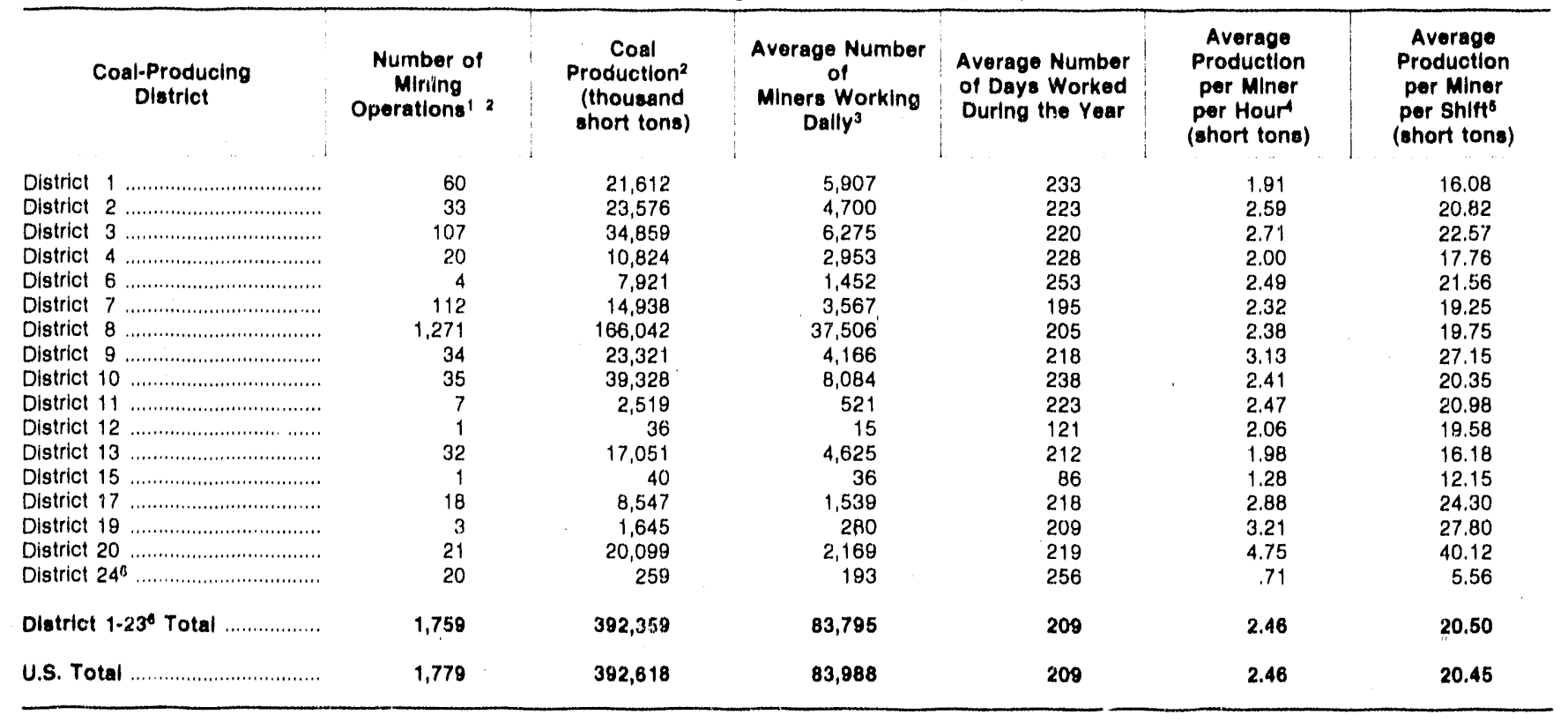

1 Includes preparation plants.

2 Excludes silt, culm, refuse barik, slurry dam, and dredge production excepl for Pennsylvania anthracite. Excludes mines producing less than 10,000 short tons of coal during the year.

3 Includes all employees engaged in production, preparation, processing, devolupment, maintenance, repair, shop or yard work at mining operailons. Excludes office workers. Includes mining operations management and all technical and engineering personnel.

4 Calculated by dividing total coal production by the total direct labor hours worked by all mine employees identified in footnote 3 above.

5 Calculated by multiplying average production per miner per hour by the average length of a miner shift.

B District 24 is the anthracite-producing district in Pennsylvania. Districts 1.23 represent the total U.S. production of bituminous, subbituminous coal, and lignite. See Appendix $C$.

Note: Total may not equal sum of components because of independent rounding.

Source: Energy Intormation Administration, Form ElA-7A, "Coal Production Report." 
Table 33. U.S. Surface Coal Mining Productivity by District, 1989

\begin{tabular}{|c|c|c|c|c|c|c|}
\hline $\begin{array}{c}\text { Coat-Producing } \\
\text { Divtrlet }\end{array}$ & $\begin{array}{l}\text { Number of } \\
\text { Mining } \\
\text { Operations } 2\end{array}$ & $\begin{array}{l}\text { Coal } \\
\text { Productlon } \\
\text { (thousand } \\
\text { hort tons) }\end{array}$ & $\begin{array}{c}\text { Average Number } \\
\text { of } \\
\text { Miners Working } \\
\text { Dally3 }\end{array}$ & $\begin{array}{l}\text { Average Number } \\
\text { of Day: Worked } \\
\text { During the Year }\end{array}$ & $\begin{array}{l}\text { Aversge } \\
\text { Production } \\
\text { per Miner } \\
\text { per Hourd } \\
\text { (ehort tons) }\end{array}$ & $\begin{array}{l}\text { Average } \\
\text { Production } \\
\text { per Miner } \\
\text { per Shifts } \\
\text { (short tons) }\end{array}$ \\
\hline 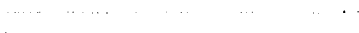 & & & & & & \\
\hline 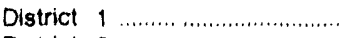 & 264 & 24,127 & 4,239 & 230 & 2.75 & 24.26 \\
\hline 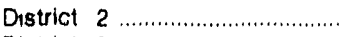 & 77 & 6.223 & 918 & 231 & 2.81 & 25.48 \\
\hline 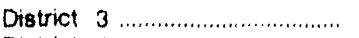 & 138 & 7.633 & 1,428 & 201 & 2.91 & 25.60 \\
\hline 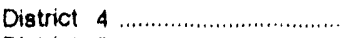 & 150 & 22.701 & 4,421 & 226 & 2.52 & 22.99 \\
\hline 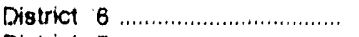 & 6 & 368 & 87 & 238 & 2.02 & 16.82 \\
\hline District $7 \ldots \ldots \ldots \ldots \ldots \ldots \ldots$ & 21 & 524 & 220 & 183 & $1.6 \theta$ & 15.04 \\
\hline 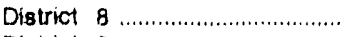 & 665 & 87,880 & 14,280 & 206 & 3.11 & 27.72 \\
\hline District 9 & 65 & 18,256 & 1,870 & 185 & 4.53 & 40.47 \\
\hline 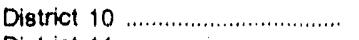 & 19 & 19,917 & 1,919 & 236 & 3.86 & 31.60 \\
\hline District 11 & 55 & 31,112 & 3,163 & 260 & 4.04 & 37.46 \\
\hline 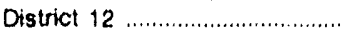 & 2 & 391 & 134 & 255 & 1.48 & 14.03 \\
\hline 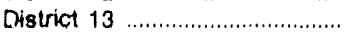 & 81 & 12,298 & 2,262 & 208 & 2.73 & 23.76 \\
\hline 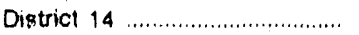 & 5 & 342 & 89 & 277 & 1.69 & 14.23 \\
\hline 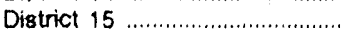 & 39 & 62,448 & 3,068 & 246 & 6.60 & 58.96 \\
\hline 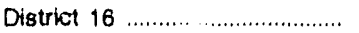 & 1 & 27 & 8 & 192 & 1.80 & 16.21 \\
\hline 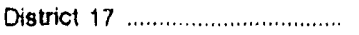 & 7 & 9,040 & 611 & 198 & 6.32 & 59.63 \\
\hline District 18 & 8 & 35,178 & 2,116 & 246 & 7.49 & 66.52 \\
\hline District 19 & 25 & 169,813 & 3,071 & 278 & 21.38 & 195.81 \\
\hline 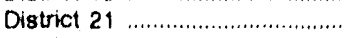 & 11 & 29,555 & 850 & 231 & 15.68 & 131.90 \\
\hline 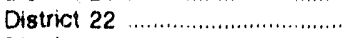 & 8 & 37,742 & 682 & 291 & 18.94 & 165.05 \\
\hline District $23 \ldots \ldots \ldots \ldots \ldots \ldots \ldots \ldots \ldots$ & 4 & $6,62 !$ & 874 & 216 & 3.86 & 33.81 \\
\hline 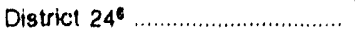 & 83 & 2,630 & 1,201 & 246 & 1.19 & 9.43 \\
\hline Dietrict $1.23^{6}$ Total ..................... & 1,649 & 582,208 & 46,308 & 217 & 5.70 & 50.67 \\
\hline 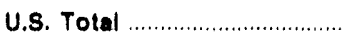 & 1,732 & 584,829 & 47,509 & 218 & 5.61 & 49.56 \\
\hline
\end{tabular}

1 Includes preparation plants.

2 Exciudes sitt, culm, refuse bank, slurry dam, and dredge production except for Pennsylvania anthracite. Excludes mines producing less than 10,000 short tons of coal during the year.

3 Includes all employees engaged in production, preparation, processing, development, maintenance, repair, shop or yard work at mining operations. Excludes office workers. Includes mining operations management and all technical and engineering personnel.

- Calculated by dividing total coal production by the total direct labor hours worked by all mine employees identifled in footnote 3 above.

5 Calculated by multiplying average production per miner per hour by the average length of a miner shift.

- District 24 is the anthracite-producing district in Pennsytvania. Districts 1.23 represent the tota! U.S. production of bituminous, subbituminous coal, and lignite. See Appendix C

Note: Total may not equal sum of components because of independent rounding.

Source: Energy Information Administration, Form ElA.7A, "Coal Production Report." 
Table 34. Average Number of Miners Working Daily ${ }^{1}$ at U.S. Coal Mines ${ }^{2}$ by State and Type of Mining, 1988, 1989

\begin{tabular}{|c|c|c|c|c|c|c|c|c|c|}
\hline \multirow{3}{*}{$\begin{array}{c}\text { Coal-Producing } \\
\text { State }\end{array}$} & \multicolumn{4}{|c|}{ Type of MIning } & \multicolumn{2}{|c|}{ Total } & \multicolumn{3}{|c|}{1988} \\
\hline & \multicolumn{2}{|c|}{ Underground } & \multicolumn{2}{|c|}{ Surface } & \multirow[b]{2}{*}{$\begin{array}{c}\text { Average } \\
\text { Number } \\
\text { of } \\
\text { Mlners } \\
\text { Working } \\
\text { Dally }\end{array}$} & \multirow[b]{2}{*}{$\begin{array}{c}\text { Percent } \\
\text { Change } \\
\text { from } \\
\text { Previous } \\
\text { Year }\end{array}$} & \multicolumn{2}{|c|}{ Type of Mining } & \multirow[b]{2}{*}{ Total } \\
\hline & $\begin{array}{c}\text { Average } \\
\text { Number } \\
\text { of } \\
\text { Miners } \\
\text { Working } \\
\text { Dally }\end{array}$ & $\begin{array}{l}\text { Porcent } \\
\text { Change } \\
\text { from } \\
\text { Prevlous } \\
\text { Year }\end{array}$ & $\begin{array}{c}\text { Average } \\
\text { Number } \\
\text { of } \\
\text { Mlners } \\
\text { Working } \\
\text { Dally }\end{array}$ & $\begin{array}{l}\text { Percent } \\
\text { Change } \\
\text { from } \\
\text { Provious } \\
\text { Yoar }\end{array}$ & & & Underground & Surface & \\
\hline 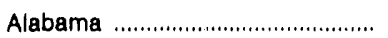 & 4,371 & -3.02 & 2,134 & -9.23 & 6,505 & -5.15 & 4,507 & 2,351 & 6,858 \\
\hline 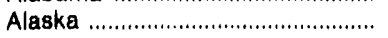 & - & - & 87 & -6.45 & 87 & -6.45 & - & 93 & 93 \\
\hline 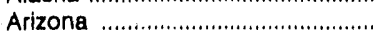 & - & - & 780 & -20.73 & 780 & -20.73 & - & 984 & 984 \\
\hline 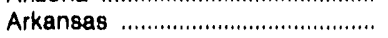 & - & - & 8 & -63.64 & 8 & -63.64 & - & 22 & 22 \\
\hline 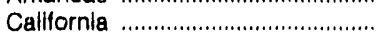 & - & - & 5 & -37.50 & 5 & -37.50 & - & 8 & 8 \\
\hline 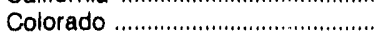 & 1,490 & 4.63 & 529 & -22.09 & 2,019 & -3.99 & 1,424 & 679 & 2,103 \\
\hline 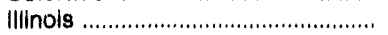 & 8,084 & 8.66 & 1,919 & -25.68 & 10,003 & -.19 & 7,440 & 2,582 & 10,022 \\
\hline 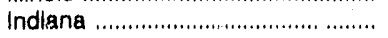 & 521 & 29.60 & 3,163 & -14.65 & 3,684 & -10.32 & 402 & 3,706 & 4,108 \\
\hline lowa & 15 & -66.67 & 134 & 76.32 & 149 & 23.14 & 45 & 76 & 121 \\
\hline 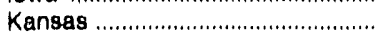 & - & - & 165 & -38.43 & 165 & -38.43 & - & 268 & 268 \\
\hline 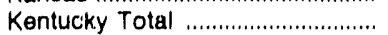 & 20,752 & 1.36 & 9,904 & -1.80 & 30,656 & .32 & 20,473 & 10,086 & 30,559 \\
\hline 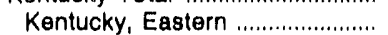 & 16,586 & 3.11 & 8,034 & -2.75 & 24,620 & 1.13 & 16,085 & 8,261 & 24,346 \\
\hline 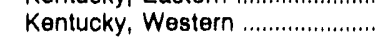 & 4,166 & -5.06 & 1,870 & 2.47 & 6,036 & -2.85 & 4,388 & 1,825 & 6,213 \\
\hline 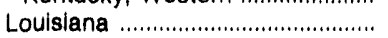 & 4,100 & - & 69 & -11.54 & 69 & -11.54 & 4,000 & 78 & 78 \\
\hline Maryland & 282 & -2.08 & 272 & 12.40 & 554 & 4.53 & 288 & 242 & 530 \\
\hline 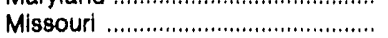 & - & - & 428 & -36.21 & 428 & -36.21 & - & 671 & 671 \\
\hline 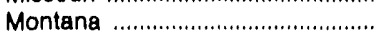 & - & - & 682 & -21.78 & 682 & -21.79 & - & 872 & 872 \\
\hline New Mexico .................................... & 49 & -69.18 & 1,421 & -.28 & 1,470 & -7.20 & 159 & 1,425 & 1,584 \\
\hline 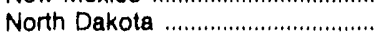 & - & - & 850 & -13.00 & 850 & -13.00 & 100 & 977 & 977 \\
\hline Ohlo & 2,953 & 3.25 & 4,421 & 4.12 & 7,374 & 3.77 & 2,860 & 4,246 & 7,106 \\
\hline 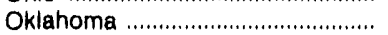 & 36 & - & 378 & -48.01 & 414 & -43.05 & 4,00 & 727 & 727 \\
\hline Pennsylvania Total ............................ & 9,578 & -6.48 & 5,891 & -6.95 & 15,469 & -6.66 & 10,242 & 6,331 & 16,573 \\
\hline Anthracite & 193 & -10.57 & 1,201 & -1.23 & 1,394 & -4.06 & $\begin{array}{r}1,246 \\
237\end{array}$ & 1,216 & 1,453 \\
\hline 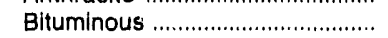 & 9,385 & -6.20 & 4,690 & -8.31 & 14,075 & -6.91 & 10,005 & 5,115 & 15,120 \\
\hline 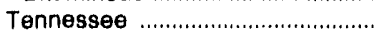 & 1,471 & -12.23 & 386 & -12.87 & 1,857 & -12.36 & 1,676 & 443 & 2,118 \\
\hline 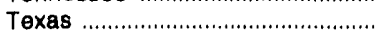 & - & - & 2,109 & -31.01 & 2,109 & -31.01 & 1,0 & 3,057 & 3,057 \\
\hline Utah & $2,16 \theta$ & -8.67 & - & - & 2,169 & -8.67 & 2,375 & 0,001 & 2,375 \\
\hline 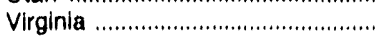 & 8,889 & -1.74 & 1,482 & -10.18 & 10,371 & -3.04 & 9,046 & 1,650 & 10,698 \\
\hline Washington & - & - & 787 & 9.92 & 787 & 9.92 & - & 716 & 716 \\
\hline 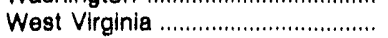 & 23,048 & -1.90 & 6,434 & 17.47 & 29,482 & 1.76 & 23,495 & 5,477 & 28,972 \\
\hline Wyoming & 280 & 112.12 & 3,071 & 1.19 & 3,351 & 5.81 & 132 & 3,035 & 3,167 \\
\hline $\begin{array}{l}\text { Appalachian Total } 4 \ldots \ldots \ldots \ldots \ldots \ldots \\
\text { Interior Total } \ldots \ldots \ldots \ldots \ldots \ldots \ldots \ldots \ldots \\
\text { Western Total } 4 \ldots \ldots \ldots \ldots \ldots \ldots \ldots \ldots \ldots\end{array}$ & $\begin{array}{r}67,178 \\
12,822 \\
3,988\end{array}$ & $\begin{array}{r}-1.50 \\
4.46 \\
-2.49\end{array}$ & $\begin{array}{r}29,054 \\
10,243 \\
8,212\end{array}$ & $\begin{array}{r}.18 \\
-21.28 \\
-6.57\end{array}$ & $\begin{array}{l}96,232 \\
23,065 \\
12,200\end{array}$ & $\begin{array}{l}-1.00 \\
-8.79 \\
-6.27\end{array}$ & $\begin{array}{r}68,199 \\
12,275 \\
4,090\end{array}$ & $\begin{array}{r}29,001 \\
13,012 \\
8,789\end{array}$ & $\begin{array}{l}97,200 \\
26,287 \\
12,878\end{array}$ \\
\hline $\begin{array}{l}\text { East of the Miss. Aiver ............. } \\
\text { Weat of the Miss. Alver ............ }\end{array}$ & $\begin{array}{r}79,949 \\
4,039\end{array}$ & $\begin{array}{r}-.60 \\
-2.32\end{array}$ & $\begin{array}{l}36,006 \\
11,503\end{array}$ & $\begin{array}{r}-2.98 \\
-15.86\end{array}$ & $\begin{array}{r}115,955 \\
15,542\end{array}$ & $\begin{array}{r}-1.35 \\
-12.80\end{array}$ & $\begin{array}{r}80,429 \\
4,135\end{array}$ & $\begin{array}{l}37,114 \\
13,688\end{array}$ & $\begin{array}{r}117,543 \\
17,823\end{array}$ \\
\hline 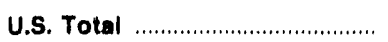 & 83,988 & -.68 & 47,509 & -6.48 & 131,497 & -2.86 & 84,564 & 50,802 & 135,366 \\
\hline
\end{tabular}

1 includes all employees engaged in production, preparation, processing, development, maintenance, repair, shop or yard work at mining operations. Excludes office workers. Includes mining operations management and all technical and engineering personnel.

2 Excludes silt, culm, refuse barik, slurry dam, and dredge production except for Pennsylvania anthracite. Excludes mines producing less than 10,000 short tons of coal during the year.

3 Percent changes are calculated using unrounded data.

4 For a definition of coal-producing regions, se日 Appendix $C$.

Source: Energy Information Administration, Form EIA.7A, "Coal Production Report." 
Table 35. Average Number of Miners Working Daily' at U.S. Coal Mines ${ }^{2}$ by District and Type of Mining, 1988, 1989

\begin{tabular}{|c|c|c|c|c|c|c|c|c|c|}
\hline \multirow{4}{*}{$\begin{array}{c}\text { Coal-Producing } \\
\text { District }\end{array}$} & ' & \multicolumn{2}{|c|}{ Type of Mining } & 989 & & al & \multicolumn{2}{|r|}{1988} & $\begin{array}{l}\text { Average Number of } \\
\text { Miners Working Dally }\end{array}$ \\
\hline & & & \multirow{2}{*}{\multicolumn{2}{|c|}{ Surface }} & . & $\therefore$ & & & \\
\hline & \multicolumn{2}{|c|}{ Underground } & & & & & \multicolumn{2}{|c|}{ Type of Mining } & \\
\hline & $\begin{array}{c}\text { Average } \\
\text { Number } \\
\text { of } \\
\text { Miners } \\
\text { Working } \\
\text { Dally }\end{array}$ & $\begin{array}{c}\text { Percent } \\
\text { Change } \\
\text { from } \\
\text { Prevlous } \\
\text { Years }\end{array}$ & $\begin{array}{c}\text { Average } \\
\text { Number } \\
\text { of } \\
\text { Miners } \\
\text { Working } \\
\text { Dally }\end{array}$ & $\begin{array}{c}\text { Percent } \\
\text { Change } \\
\text { from } \\
\text { Previous } \\
\text { Year }\end{array}$ & $\begin{array}{c}\text { Average } \\
\text { Numbor } \\
\text { of } \\
\text { Miners } \\
\text { Worklng } \\
\text { Dally }\end{array}$ & $\begin{array}{l}\text { Percent } \\
\text { Change } \\
\text { from } \\
\text { Prevlous } \\
\text { Year }\end{array}$ & Underground & Surtace & Total \\
\hline 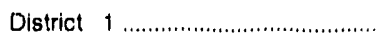 & 5,907 & $-4,63$ & 4,239 & -3.86 & 10,140 & $-4,31$ & 6,194 & 4,409 & 10,603 \\
\hline 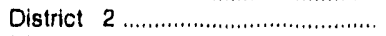 & 4,700 & -5.72 & 918 & -23.50 & 5,618 & -9.17 & 4,985 & 1,200 & 6,185 \\
\hline 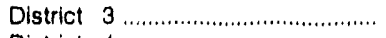 & 6,275 & -.63 & 1,426 & 21.47 & 7,701 & 2.83 & 6,315 & 1,174 & 7,489 \\
\hline 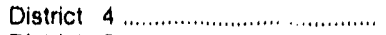 & 2,953 & 3.2 .5 & 4,421 & 4.12 & 7,374 & 3.77 & 2,860 & 4,246 & 7,106 \\
\hline 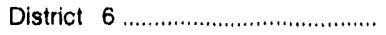 & 1,452 & 1.89 & 87 & 26.09 & 1,539 & 3.01 & 1,425 & 69 & 1,494 \\
\hline 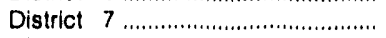 & 3,567 & -8.28 & 220 & 17.02 & 3,787 & -7.11 & 3,809 & 188 & 4,077 \\
\hline 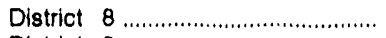 & 37,506 & .09 & 14,280 & 1.80 & 51,786 & .56 & 37,471 & 14,028 & 51,499 \\
\hline 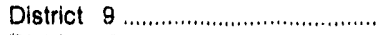 & 4,166 & -5.06 & 1,870 & 2.47 & 6,036 & -2.85 & 4,388 & 1,825 & 6,213 \\
\hline 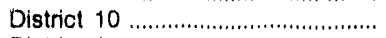 & 8,084 & 8.66 & 1,019 & -25.68 & 10,003 & -.19 & 7,440 & 2,582 & 10,022 \\
\hline 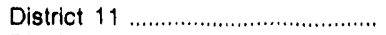 & 521 & 29.60 & 3,163 & -14.65 & 3,684 & -10.32 & 402 & 3,706 & 4,108 \\
\hline 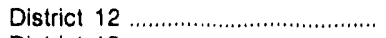 & 15 & -66.67 & 134 & 76.32 & 148 & 23.14 & 45 & 76 & 121 \\
\hline 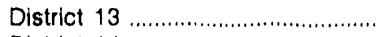 & 4,625 & -4.11 & 2,262 & -8.46 & 6,887 & -5.58 & 4,823 & 2,471 & 7,294 \\
\hline 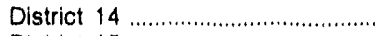 & - & - & 89 & -49.43 & 89 & -49.43 & - & 176 & 176 \\
\hline 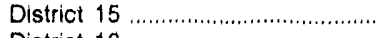 & 36 & - & 3,068 & -33.88 & 3,104 & -33.20 & - & 4,647 & 4,647 \\
\hline 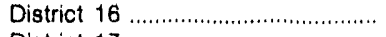 & - & - & 8 & -66.87 & 8 & -66.67 & - & 24 & 24 \\
\hline 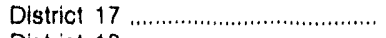 & 1,539 & -2.78 & 611 & -22.66 & 2,150 & -9.40 & 1,583 & 790 & 2,373 \\
\hline 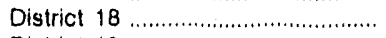 & - & - & 2,116 & -7.27 & 2,116 & -7.27 & - & 2,282 & 2,282 \\
\hline 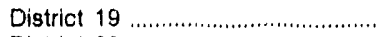 & 280 & 112.12 & 3,071 & 1.19 & 3,351 & 5.81 & 132 & 3,035 & 3,167 \\
\hline 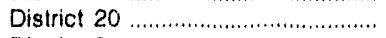 & 2,169 & -8.67 & - & - & $2,16 \theta$ & -8.67 & 2,375 & - & 2,375 \\
\hline 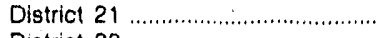 & - & - & 850 & -13.00 & 850 & -13.00 & - & 977 & 977 \\
\hline 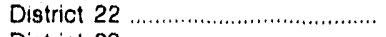 & - & - & 682 & -21.79 & 682. & -21.79 & - & 872 & 872 \\
\hline 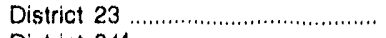 & - & - & 874 & 8.03 & 874 & 8.03 & - & 809 & 809 \\
\hline 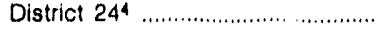 & 193 & -18.57 & 1,201 & -1.23 & 1,394 & -4.06 & 237 & 1,216 & 1,453 \\
\hline 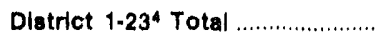 & 83,795 & -.63 & 46,308 & -6.61 & $93 n, 103$ & -2.85 & 64,327 & 49,586 & 133,813 \\
\hline U.S. Total & 83,988 & -.68 & 47,509 & -6.48 & 131,497 & -2.86 & 84,564 & 50,802 & 135,386 \\
\hline
\end{tabular}

1 Includes all employees engaged in production, preparation, processing, development, maintenance, repair, shop or yard work at mining operations. Ex. cludes office workers. Includes mining operations management and all technical and engineering personnel.

2 Excludes silt, culm, refuse bank, slurry dam, and dredge productioni except for Pennsylvania anthracite. Excludes mines producing less than 10,000 short tons of coal during the year.

3 Percent changes are calculated using unrounded data.

4 District 24 is the anthracite-producing district in Pennsylvania. Districts 1.23 represent the total U.S. production of bituminous, subbituminous coal, and lignite. See Appenaix. C.

Source: Energy Information Administration, Form EIA-7A, "Coal Production Repon." 
Table 36. Average Number of Miners Working Daily ${ }^{1}$ at U.S. Coal Mines ${ }^{2}$ by Mine Production Range and Type of Mining, 1988, 1989

\begin{tabular}{|c|c|c|c|c|c|c|c|c|c|}
\hline \multirow{3}{*}{$\begin{array}{l}\text { Mine } \\
\text { Production Range } \\
\text { (Thoueanc Short Tons) }\end{array}$} & \multicolumn{4}{|c|}{ Type of Mining } & \multicolumn{2}{|c|}{ Total } & \multicolumn{3}{|c|}{$\begin{array}{l}\text { Average Number of } \\
\text { Miners Working Dally }\end{array}$} \\
\hline & \multicolumn{2}{|c|}{ Underground } & \multicolumn{2}{|c|}{ Surface } & \multirow[b]{2}{*}{$\begin{array}{c}\text { Average } \\
\text { Number } \\
\text { of } \\
\text { Miners } \\
\text { Working } \\
\text { Dally }\end{array}$} & \multirow[b]{2}{*}{$\begin{array}{c}\text { Percent } \\
\text { Change } \\
\text { from } \\
\text { Prevlous } \\
\text { Year3 }\end{array}$} & \multicolumn{2}{|c|}{ Type of Mining } & \multirow[b]{2}{*}{ Total } \\
\hline & $\begin{array}{c}\text { Average } \\
\text { Nuinber } \\
\text { of } \\
\text { Miners } \\
\text { Working } \\
\text { Dally }\end{array}$ & $\begin{array}{l}\text { Percent } \\
\text { Change } \\
\text { from } \\
\text { Prevlous } \\
\text { Year }\end{array}$ & $\begin{array}{c}\text { Average } \\
\text { Number } \\
\text { of } \\
\text { Miners } \\
\text { Working } \\
\text { Dally }\end{array}$ & $\begin{array}{c}\text { Percent } \\
\text { Change } \\
\text { from } \\
\text { Previous } \\
\text { Year }{ }^{3}\end{array}$ & & & Underground & Surface & \\
\hline Over $1000 \ldots \ldots$ & 34,895 & 6.97 & 16,973 & -9.91 & 51,868 & 0.79 & 32,620 & 18,840 & 51,460 \\
\hline 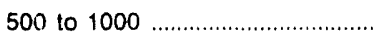 & 11,393 & -9.45 & 6,133 & -9.11 & 17,526 & -9.33 & 12,582 & 6,748 & 19,330 \\
\hline 200 to $500 \ldots$ & 11,099 & -4.25 & 8,046 & 3.54 & 19,145 & -1.13 & 11,592 & 7,771 & 19,363 \\
\hline 100 to $200 \ldots$ & 7,513 & -9.61 & 4,447 & -11.99 & 11,960 & -10.51 & 8,312 & 5,053 & 13,365 \\
\hline 50 to 100 & 5,832 & -1.92 & $4,05 ?$ & -3.91 & 9,884 & -2.75 & 5,946 & $4,21 i$ & 10,163 \\
\hline 10 to 50 & 6,229 & -7.83 & 4,610 & -11.58 & 10,839 & -9.46 & 6,758 & 5,214 & 11,972 \\
\hline 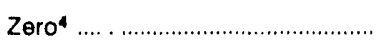 & 7,027 & 4.04 & 3,248 & 9.77 & 10,275 & 5.79 & 6,754 & 2,959 & 9,713 \\
\hline U.S. Total & 83,988 & -.68 & 47,509 & -6.48 & 131,497 & -2.86 & 84,564 & 50,802 & 135,366 \\
\hline
\end{tabular}

1 Includes all employees engaced in production, preparaticn, processing, development, maintenance, repair, shop or yard work at mining operations. Excludes office workers. Includes minir 7 operations management and all technical and engineering personnel.

2 Excludes silt, culm, refuse bank, slurry dam, and dredge production except for Pennsylvania anthracite. Excludes mines producing less than 10,000 short tons of coal during the year.

3 Percent changes are calculated using unrounded data.

- Includes all employees at preperation plants and tipples not co-located with a mine.

Source: Energy information Administration, Form EIA.7A, "Coal Production Report." 
Table 37. U.S. Coal Mining ${ }^{1}$ Productivity ${ }^{2}$ by State and Type of Mining, 1988, 1989

(Short Tons of Coal Produced Per Miner Hour)

\begin{tabular}{|c|c|c|c|c|c|c|c|c|c|}
\hline \multirow{4}{*}{$\begin{array}{l}\text { Coal-Producing } \\
\text { state }\end{array}$} & \multicolumn{6}{|c|}{1989} & \multicolumn{3}{|c|}{1988} \\
\hline & \multicolumn{4}{|c|}{ Type of Mining } & \multicolumn{2}{|c|}{ Total } & \multicolumn{3}{|c|}{ Productivity } \\
\hline & \multicolumn{2}{|c|}{ Underground } & \multicolumn{2}{|c|}{ Surface } & \multirow[b]{2}{*}{ Productivity } & \multirow[b]{2}{*}{$\begin{array}{l}\text { Percent } \\
\text { Change } \\
\text { from } \\
\text { Previous } \\
\text { Year }\end{array}$} & \multicolumn{2}{|c|}{ Type of Mining } & \multirow[b]{2}{*}{ Total } \\
\hline & Productivity & $\begin{array}{c}\text { Percent } \\
\text { Change } \\
\text { front } \\
\text { Provlous } \\
\text { Year }^{3}\end{array}$ & Productlvity & $\begin{array}{c}\text { Persent } \\
\text { Change } \\
\text { trom } \\
\text { Provious } \\
\text { Year }^{3}\end{array}$ & & & Underground & Surface & \\
\hline Alabama & 2.07 & 13.79 & 2.72 & 7.89 & 2.25 & 10.85 & 1.76 & 2.52 & 2.03 \\
\hline Alaska & - & - & 7.73 & -4.66 & 7.73 & -4.66 & - & 8.11 & 8.11 \\
\hline Arizona . .............................. & - & - & 6.25 & -3.10 & 6.25 & -3.10 & - & 6.45 & 6.45 \\
\hline 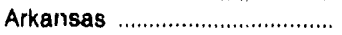 & - & - & 1.75 & -77.22 & 1.75 & -77.22 & - & 7.67 & 7.67 \\
\hline 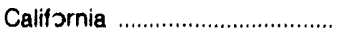 & - & - & 8.21 & -45.02 & 8.21 & -45.02 & - & 14.93 & 14.93 \\
\hline 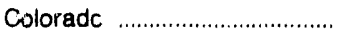 & 2.91 & 12.39 & 6.74 & 3.53 & 4.08 & 3.48 & 2.59 & 6.51 & 3.94 \\
\hline Illinois & 2.41 & -.81 & 3.96 & 19.30 & 2.77 & 3.73 & 2.43 & 3.32 & 2.67 \\
\hline 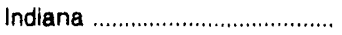 & 2.47 & 8.37 & 4.04 & 9.77 & 3.86 & 9.75 & 2.28 & 3.68 & 3.52 \\
\hline lowa ……………………… & 2.06 & - & 1.48 & -5.32 & 1.51 & -2.73 & 0 & 1.56 & 1.55 \\
\hline 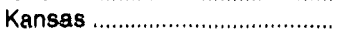 & - & - & 2.66 & 82.04 & 2.66 & 82.04 & - & 1.46 & 1.46 \\
\hline Kentucky Total ........................... & 2.54 & .48 & 3.23 & 2.79 & 2.78 & 1.47 & 2.53 & 3.14 & 2.7 .4 \\
\hline Kentucky, Eastern ..................... & 2.40 & -.93 & 2.92 & -7.09 & 2.58 & -3.48 & 2.42 & 3.14 & 2.68 \\
\hline Kentucky, Western ................ & 3.13 & 9.57 & 4.53 & 44.59 & 3.62 & 22.75 & 2.86 & 3.13 & 2.95 \\
\hline Louisiana .............................. & - & - & 15.04 & -6.07 & 15.04 & -6.07 & - & 16.01 & 16.01 \\
\hline Maryland .............................. & 3.09 & -9.89 & 2.90 & 13.51 & 3.00 & -1.31 & 3.43 & 2.56 & 3.04 \\
\hline Missouri ……………………. & - & - & 3.43 & 28.06 & 3.43 & 28.06 & - & 2.68 & 2.68 \\
\hline 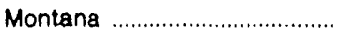 & - & - & 18.94 & -3.17 & 18.84 & -3.17 & - & 19.56 & 19.56 \\
\hline New Mexico ................................ & 1.03 & -52.08 & 8.03 & 21.49 & 7.93 & 22.50 & 2.14 & 6.61 & 6.47 \\
\hline North Dakota .............................. & - & - & $15.6 \theta$ & .35 & 15.68 & .35 & - & 15.63 & 15.63 \\
\hline Ohio ………………………. & 2.00 & 2.38 & 2.52 & -6.46 & 2.33 & -2.82 & 1.95 & 2.70 & 2.39 \\
\hline Oklahoma & 1.28 & - & 2.18 & 3.98 & 2.15 & 2.31 & - & 2.10 & 2.10 \\
\hline Pennsylvania Total ..................... & 2.16 & 9.65 & 2.47 & 6.44 & 2.28 & 8.30 & 1.97 & 2.32 & 2.11 \\
\hline 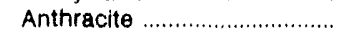 & .71 & -16.56 & 1.10 & -6.89 & 1.12 & -7.31 & .86 & 1.28 & 1.21 \\
\hline Bituminous ………………............ & 2.19 & 9.86 & 2.76 & 8.98 & 2.39 & 9.46 & 1.99 & 2.53 & 2.18 \\
\hline Tennessee ............................... & 1.58 & 8.94 & 2.20 & 9.52 & 1.72 & 9.00 & 1.45 & 2.00 & 1.57 \\
\hline 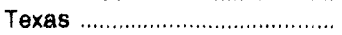 & - & - & 7.31 & 12.18 & 7.31 & 12.18 & - & 6.52 & 6.52 \\
\hline 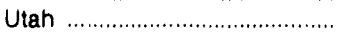 & 4.75 & 14.20 & - & - & 4.75 & 14.20 & 4.16 & -. & 4.16 \\
\hline Virginia & 2.15 & -5.71 & 2.59 & 3.88 & 2.21 & -4.53 & 2.28 & 2.49 & 2.31 \\
\hline 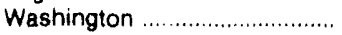 & - & - & 3.34 & -6.58 & 3.34 & -6.58 & - & 3.57 & 3.57 \\
\hline West Virginia ........................... & 2.59 & 4.00 & 3.49 & -1.17 & 2.77 & 3.66 & 2.49 & 3.53 & 2.68 \\
\hline 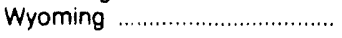 & 3.21 & -13.78 & 21.38 & 10.50 & 20.28 & 7.86 & 3.72 & 19.34 & 18.80 \\
\hline Appolachisn Totar $4 \ldots \ldots \ldots \ldots$ & 2.34 & 3.02 & 2.84 & -.08 & 2.48 & $\begin{array}{r}2.07 \\
11.32\end{array}$ & 2.27 & 2.84 & 2.44 \\
\hline $\begin{array}{l}\text { Interlor Totat4 } \\
\text { Western Total4 }\end{array}$ & $\begin{array}{l}2.63 \\
3.93\end{array}$ & $\begin{array}{r}2.12 \\
10.58\end{array}$ & $\begin{array}{r}4.97 \\
14.64\end{array}$ & $\begin{array}{r}18.1 \\
6.89\end{array}$ & $\begin{array}{r}3.84 \\
11.63\end{array}$ & $\begin{array}{r}11.32 \\
5.63\end{array}$ & $\begin{array}{l}2.57 \\
3.55\end{array}$ & $\begin{array}{r}4.20 \\
13.70\end{array}$ & $\begin{array}{r}3.45 \\
11.01\end{array}$ \\
\hline East of the Mises. River ......... & 2.39 & 2.78 & 3.13 & 4.77 & 2.63 & 3.62 & 2.32 & 2.99 & 2.54 \\
\hline West of the Miss. River ....... & 3.82 & 10.17 & 11.86 & 10.53 & 10.21 & 8.84 & 3.55 & 10.73 & 8.38 \\
\hline U.S. Total & 2.46 & 3.43 & 5.61 & 5.35 & 3.70 & 4.23 & 2.38 & 5.32 & 3.55 \\
\hline
\end{tabular}

1 Excludes silt, culm, refuse bank, slurry dam, and dredge production except for Pennsylvania ant'nracite. Excludes mines producing less thar 10,000 short tons of coal during the year.

2 Calculated by dividing total coal production by the total direct labor hours worked by all employees engaged in production, preparation, processing, de. velopment. mairtenance, repair, and shop or yero work at mining operations. Excludes office workers. Includes mining operations management and all technical and engineering personnel.

- Percent changes are calculated using unrounded data.

4 For a definition of coal-producing regions, see Appendix $C$ Source: Energy Information Administration, Form ElA-7A, "Coal Production Repori.." 
Table 38. U.S. Coal Mining ${ }^{1}$ Productivity ${ }^{2}$ by District and Type of Mining, 1988, 1989

(Short Tons of Coal Froduced Per Miner Hour)

\begin{tabular}{|c|c|c|c|c|c|c|c|c|c|}
\hline \multirow{4}{*}{$\begin{array}{l}\text { Coal-Producing } \\
\text { District }\end{array}$} & \multicolumn{6}{|c|}{1889} & \multicolumn{3}{|c|}{1988} \\
\hline & \multicolumn{4}{|c|}{ Type of Mining } & \multicolumn{2}{|c|}{ Total } & \multicolumn{3}{|c|}{ Productivity } \\
\hline & Undergr & ound & Surfa & & & & Type of & lining & \\
\hline & Productivity & $\begin{array}{c}\text { Percent } \\
\text { Change } \\
\text { from } \\
\text { Provlous } \\
\text { Year }\end{array}$ & Productivity & $\begin{array}{c}\text { Percent } \\
\text { Change } \\
\text { from } \\
\text { Previous } \\
\text { Year }\end{array}$ & Productlvity & $\begin{array}{l}\text { Percent } \\
\text { Change } \\
\text { from } \\
\text { Previous } \\
\text { Year }\end{array}$ & Underground & Surface & Total \\
\hline 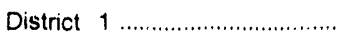 & 1.91 & 11.18 & 2.75 & 10.74 & 2.28 & 11.41 & 1.72 & 2.48 & 2.04 \\
\hline 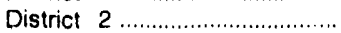 & $2.5 \theta$ & 9.09 & 2.91 & 7.90 & 2.65 & 8.38 & 2.37 & 2.70 & 2.44 \\
\hline 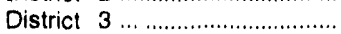 & 2.71 & .56 & 2.91 & -19.82 & 2.74 & -2.34 & 2.69 & 3.63 & 2.81 \\
\hline 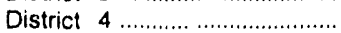 & 2.00 & 2.38 & 2.52 & -6.46 & 2.33 & -2.82 & 1.95 & 2.70 & 2.39 \\
\hline District 6 & 2.49 & 1.53 & 2.02 & -12.09 & 2.47 & .73 & 2.45 & 2.30 & 2.45 \\
\hline 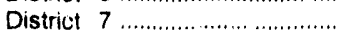 & 2.32 & 1.11 & 1.69 & -11.07 & 2.29 & .48 & 2.30 & 1.90 & 2.28 \\
\hline 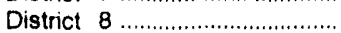 & 2.38 & .47 & 3.11 & -1.62 & 2.59 & -.06 & 2.37 & 3.16 & 2.59 \\
\hline District 9 & 3.13 & 9.57 & 4.53 & 44.59 & 3.62 & 22.75 & 2.86 & 3.13 & 2.95 \\
\hline District 10 & 2.41 & -.81 & 3.96 & 19.30 & 2.77 & 3.73 & 2.43 & 3.32 & 2.67 \\
\hline 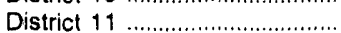 & 2.47 & 8.37 & 4.04 & 9.77 & 3.86 & 9.75 & 2.28 & 3.68 & 3.52 \\
\hline District 12 & 2.06 & 0.01 & 1.48 & -5.32 & 1.51 & -2.73 & 0 & 1.56 & 1.55 \\
\hline District 13 & 1.98 & 12.66 & 2.73 & 8.07 & 2.24 & 10.36 & 1.76 & 2.53 & 2.03 \\
\hline 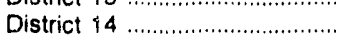 & - & 12.00 & 1.69 & -30.00 & 1.69 & -30.00 & - & 2.42 & 2.42 \\
\hline 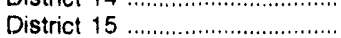 & 1.28 & - & 6.60 & 17.68 & 6.58 & 17.37 & - & 5.61 & 5.61 \\
\hline District 16 & - & - & 1.80 & -4.73 & 1.80 & -4.73 & - & 1.89 & 1.89 \\
\hline District 17 & 2.88 & 12.05 & 6.32 & 5.97 & 4.00 & 4.66 & 2.57 & 5.97 & 3.83 \\
\hline District 18 & - & 12.00 & 7.49 & 10.60 & 7.49 & 10.60 & - & 6.78 & 6.78 \\
\hline District 19 & 3.21 & -13.78 & 21.38 & 10.50 & 20.28 & 7.86 & 3.72 & 19.34 & 18.80 \\
\hline District 20 & 4.75 & 14.20 & - & - & 4.75 & 14.20 & 4.16 & - & 4.16 \\
\hline District 21 & - & - & 15.69 & .35 & 15.69 & .35 & - & 15.63 & 15.63 \\
\hline District 22 & - & - & 18.94 & -3.17 & 18.94 & -3.17 & - & 19.56 & 18.56 \\
\hline District 23 & - & - & 3.86 & -7.16 & 3.86 & -7.16 & - & 4.16 & 4.16 \\
\hline District 244 & .71 & -16.56 & 1.19 & -6.89 & 1.12 & -7.31 & .86 & 1.28 & 1.21 \\
\hline Dlatrict $1-23^{4}$ Total .................. & 2.46 & 3.43 & 5.70 & 5.49 & 3.73 & 4.28 & 2.38 & 5.41 & 3.58 \\
\hline 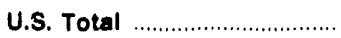 & 2.46 & 3.43 & 5.61 & 5.35 & 3.70 & 4.23 & 2.38 & 5.32 & 3.55 \\
\hline
\end{tabular}

1 Excludes silt, culm, refuse bank, slurry dam, and dredge production, except for $\mathrm{Pe}_{\mathrm{\theta}}$ : sylvania anthracite. Excludes mines producing less than 10,000 short tons of coal during the year.

2 Calculated by dividing total coal production by the total direct labor hours worked by all emplcyees engaged in production, preparation, processing, de. velopment, maintenance, repair, and shop or yard work at mining operations. Excludes sffice workers. Includes mining operations management and all technical and engineering personnel.

3 Percent changes are calculated using unrounded data.

4 District 24 is the anthracite-producing district in Pennsylvania. Districts 1.23 represent the total U.S. production of bituminous, subbituminous coal, and lignite. See Appendix C.

Source: Energy Information Administration, Form EIA-7A. "Coal Production Report." 


\section{Recoverable Reserves}

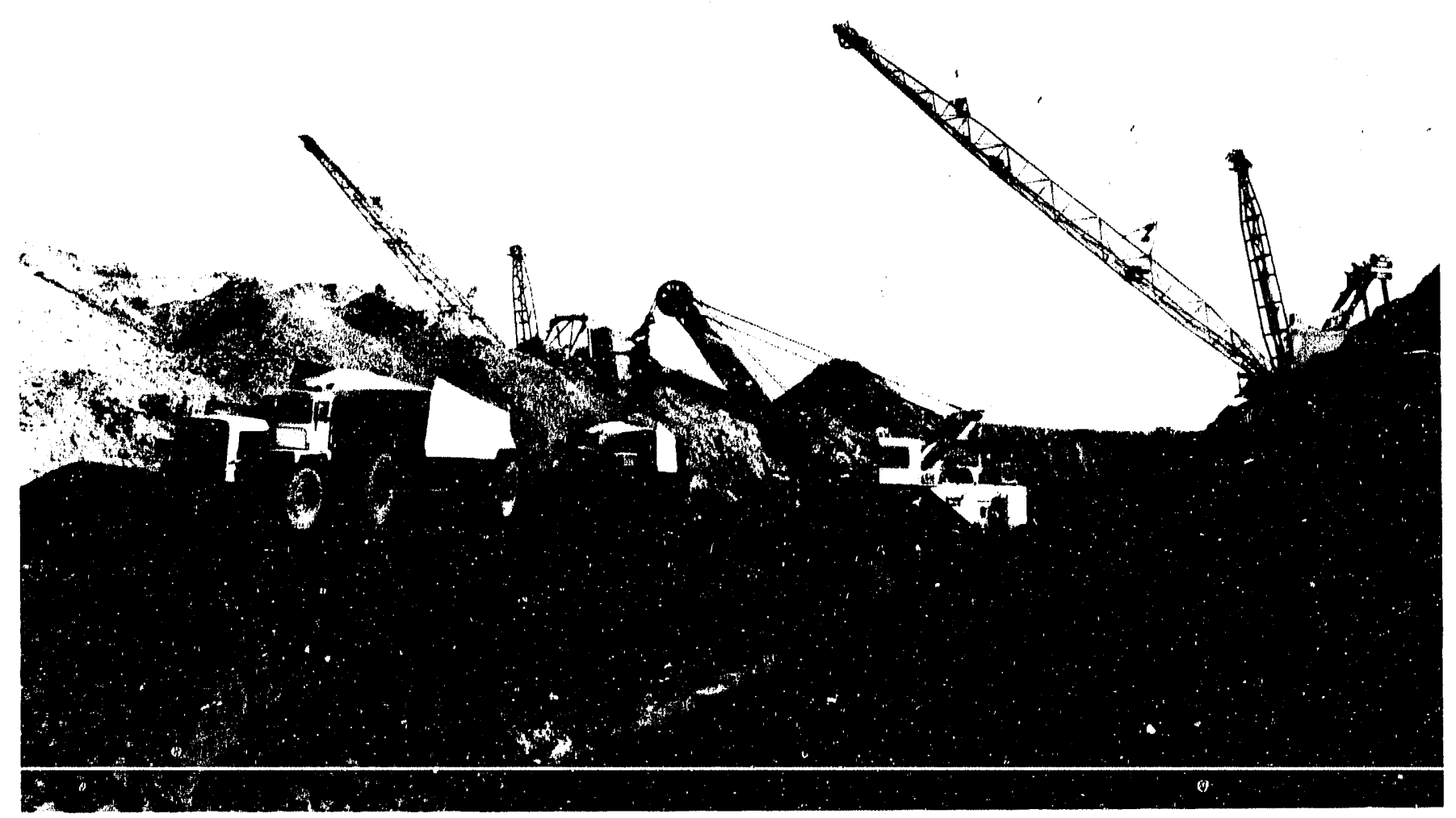

Large capacity off-road trucks are being used to haul Texas lignite. 
Figure 7. Recoverable Coal Reserves at U.S. Mines by Type of Mining and by Region, 1980-1989
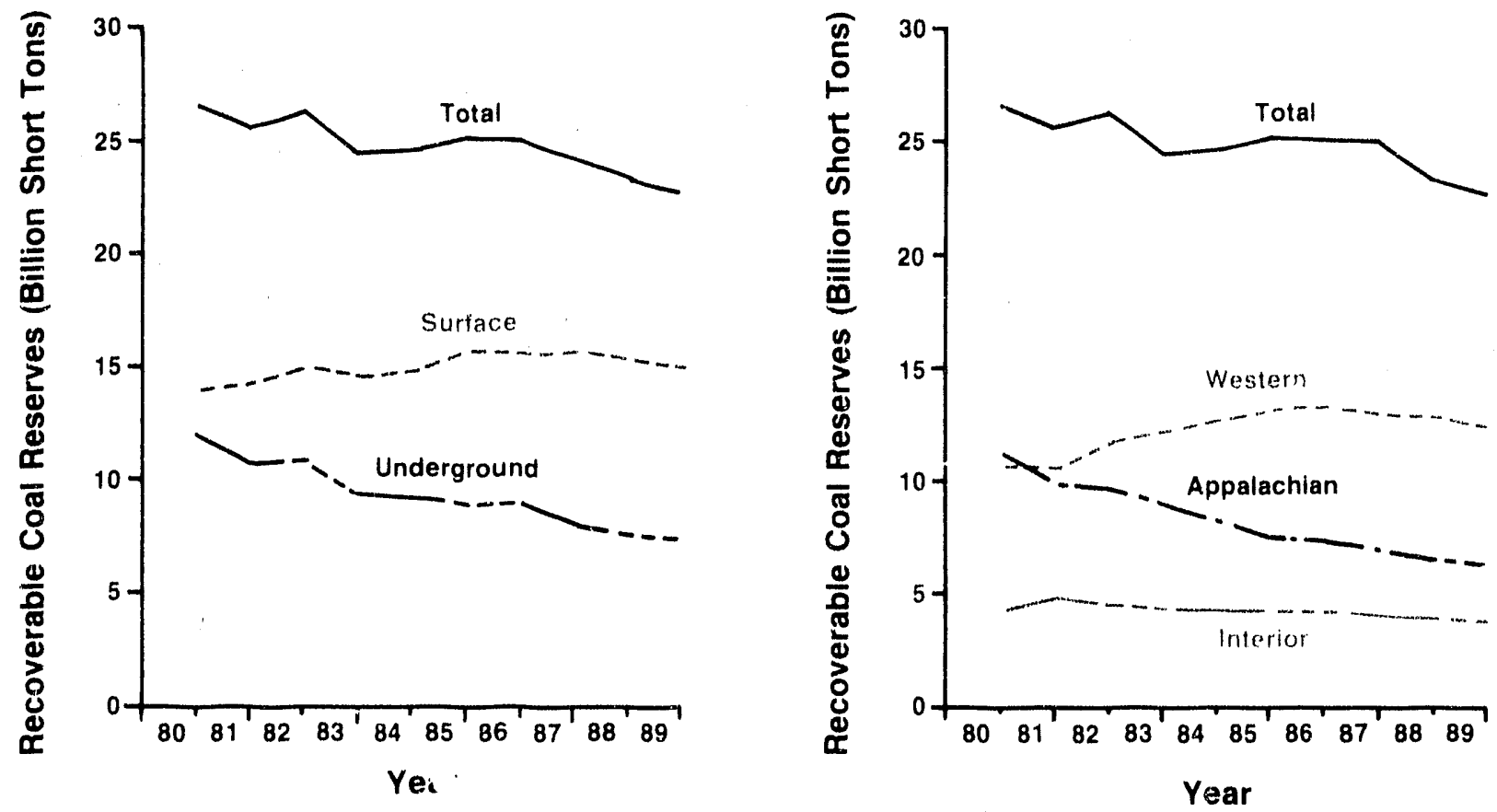

Source: Energy Information Administration, Form ElA-7A, "Coal Production Report." 


\section{Table 39. Recoverable Coal Reserves ${ }^{1}$ and Average Recovery Percentage ${ }^{2}$ at U.S. Mines ${ }^{3}$ by State and Type of Mining, 1989}

(Million Short Tons)

\begin{tabular}{|c|c|c|c|c|c|c|}
\hline \multirow{3}{*}{$\begin{array}{l}\text { Coal-Producing } \\
\text { State }\end{array}$} & \multicolumn{4}{|c|}{ Type vi Mlning } & \multicolumn{2}{|c|}{ Total } \\
\hline & \multicolumn{2}{|c|}{ Underground } & \multicolumn{2}{|c|}{ Surface } & \multirow[b]{2}{*}{$\begin{array}{l}\text { Recoverable } \\
\text { Coal Peserves }\end{array}$} & \multirow[b]{2}{*}{$\begin{array}{l}\text { Average } \\
\text { Recovery } \\
\text { Percentage }\end{array}$} \\
\hline & $\begin{array}{l}\text { Recoverable } \\
\text { Coal Reserves }\end{array}$ & $\begin{array}{c}\text { Average } \\
\text { Pecovery } \\
\text { Percentago }\end{array}$ & $\begin{array}{l}\text { Recoverabie } \\
\text { Coal Reserves }\end{array}$ & $\begin{array}{c}\text { Average } \\
\text { Recovery } \\
\text { Percentage }\end{array}$ & & \\
\hline 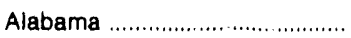 & 359.2 & 56.18 & 148.6 & 86.46 & 507.7 & 81.00 \\
\hline 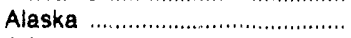 & - & - & $w$ & $w$ & w & $w$ \\
\hline Arizona & - & - & $w$ & $w$ & $w$ & $w$ \\
\hline 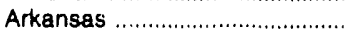 & - & - & $w$ & $w$ & $w$ & $w$ \\
\hline 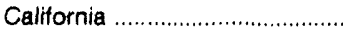 & - & - & $w$ & $w$ & $w$ & $w$ \\
\hline 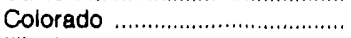 & 476.9 & 61.57 & 185.1 & 88.00 & 662.1 & 69.50 \\
\hline 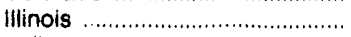 & $1,225.4$ & 46.97 & 155.3 & 78.13 & $1,380.7$ & 57.59 \\
\hline 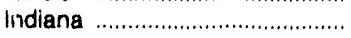 & 78.0 & 53.00 & 396.9 & 82.56 & 474.9 & 79.48 \\
\hline 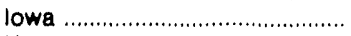 & - & - & w & $w$ & $w$ & $w$ \\
\hline 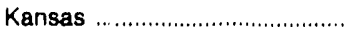 & - & - & 27.6 & 78.25 & 27.6 & 78.25 \\
\hline Kentucky Tota! ............................... & $1,012.0$ & 62.75 & 501.2 & 82.98 & $1,513.2$ & 70.48 \\
\hline Kentucky, Eastern .................... & 615.3 & 64.00 & 317.8 & 81.95 & 833.1 & 70.26 \\
\hline 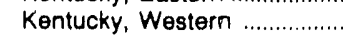 & 396.7 & 48.33 & 183.3 & 87.03 & 580.1 & 72.05 \\
\hline 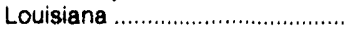 & - & - & $w$ & $w$ & $w$ & $w$ \\
\hline 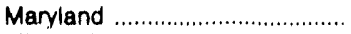 & $w$ & $w$ & $w$ & $w$ & 86.7 & 82.76 \\
\hline 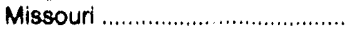 & - & - & $w$ & $w$ & w & $w$ \\
\hline 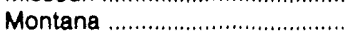 & - & - & $1,513.7$ & 91.00 & $1,513,7$ & 91.00 \\
\hline New Mexico .................................. & $w$ & $w$ & $w$ & $w$ & $1,410.7$ & 86.71 \\
\hline North Dakota & - & - & $1,360.6$ & 80.30 & $1,360.6$ & 89.30 \\
\hline Ohio & 460.4 & 60.25 & 354.2 & 86.69 & 814.6 & 84.30 \\
\hline 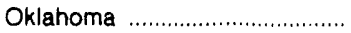 & $w$ & $w$ & $w$ & $w$ & 23.7 & 84.60 \\
\hline Ponnsylvania Total ....................... & 824.5 & 65.50 & 359.6 & 80.73 & $1,184.1$ & 77.25 \\
\hline Anthracite $\ldots \ldots \ldots \ldots \ldots \ldots \ldots$ & 34.1 & 71.25 & 26.0 & 61.38 & 60.1 & 63.51 \\
\hline 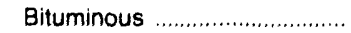 & 790.4 & 64.73 & 333.6 & 83.54 & $1,124.0$ & 79.20 \\
\hline 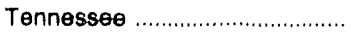 & $w$ & $w$ & $w$ & $w$ & 71.6 & 69.54 \\
\hline 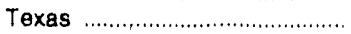 & - & - & $1,135.3$ & 83.43 & $1,135.3$ & 83.43 \\
\hline 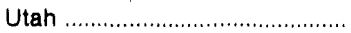 & 518.6 & 56.16 & - & - & 518.6 & 56.16 \\
\hline 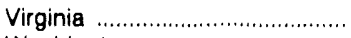 & 353.5 & 64.07 & 66.2 & 84.66 & 419.7 & 68.38 \\
\hline 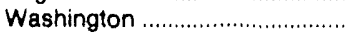 & - & - & w & $w$ & w & w \\
\hline 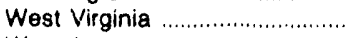 & $1,889.7$ & 62.61 & 423.7 & 80.88 & $2,313.4$ & 68.84 \\
\hline 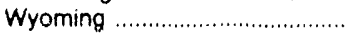 & w & $w$ & $w$ & $w$ & $6,287.7$ & 87.18 \\
\hline Appalachian Totak 4 ................... & $w$ & 63.48 & $\mathbf{w}$ & 82.67 & $6,330.8$ & 72.63 \\
\hline Intorlor Total 4 & $w$ & 48.36 & $w$ & 83.58 & $3,906.6$ & 73.14 \\
\hline 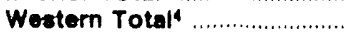 & $1,092.1$ & 58.86 & $11,350.2$ & 89.31 & $12,442.3$ & 77.93 \\
\hline $\begin{array}{l}\text { East of the Miss. Rlver ........ } \\
\text { West of the Miss. River ...... }\end{array}$ & $\begin{array}{l}6,300.2 \\
1,092.3\end{array}$ & $\begin{array}{l}62.48 \\
59.05\end{array}$ & $\begin{array}{r}2,466.4 \\
12,820.9\end{array}$ & $\begin{array}{l}82.77 \\
86.88\end{array}$ & $\begin{array}{r}8,766.6 \\
13,913.2\end{array}$ & $\begin{array}{l}72.42 \\
79.53\end{array}$ \\
\hline 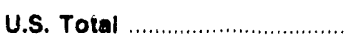 & $7,392.5$ & 62.34 & $15,287.3$ & 83.22 & $22,679.8$ & 72.86 \\
\hline
\end{tabular}

1 Represents the quantity of coal that can be recovered (i.e. mined) from existing coal reserves at reporting mines.

2 Represents the percentage of coal that can be recovered from coal reserves at reporting mines, averaged tor all mines in the reported geographic area.

${ }^{3}$ Excludes silt, culm, refuse bank, slurry dam, and dredge operations. Excludes mines producing less than 10,000 short tons of coal during the year.

- For a definition of coal-producing regions, see Appendix $C$.

- Withheld to avoid disclosure of individual company data.

Note: Total may not equal sum of components bacause of independent rounding.

Source: Energy Information Administration, Form EIA.7A "Coal Production Report." 
Table 40. Recoverable Coal Reserves ${ }^{1}$ and Average Recovery Percentage ${ }^{2}$ at U.S. Mines ${ }^{3}$ by District and Type of Mining, 1989 (Million Short Tons)

\begin{tabular}{|c|c|c|c|c|c|c|}
\hline \multirow{3}{*}{$\begin{array}{c}\text { Coal-Producing } \\
\text { District }\end{array}$} & \multicolumn{4}{|c|}{ Type of Mining } & \multicolumn{2}{|c|}{ Total } \\
\hline & \multicolumn{2}{|c|}{ Underground } & \multicolumn{2}{|c|}{ Surface } & \multirow[b]{2}{*}{$\begin{array}{l}\text { Recoverable } \\
\text { Coal Reserves }\end{array}$} & \multirow[b]{2}{*}{$\begin{array}{c}\text { Aversge } \\
\text { Recovery } \\
\text { Percentage }\end{array}$} \\
\hline & $\begin{array}{l}\text { Recoverable } \\
\text { Coal Resurves }\end{array}$ & $\begin{array}{l}\text { Average } \\
\text { Recovery } \\
\text { Percentage }\end{array}$ & $\begin{array}{l}\text { Recoverable } \\
\text { Coal Reserves }\end{array}$ & $\begin{array}{c}\text { Average } \\
\text { Recovery } \\
\text { Percentage }\end{array}$ & & \\
\hline District $1 \ldots \ldots \ldots \ldots \ldots \ldots$ & $w$ & $w$ & $w$ & $w$ & 811.1 & 79.78 \\
\hline 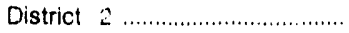 & 468.2 & 67.91 & 36.9 & 81.87 & 505.1 & 77.64 \\
\hline 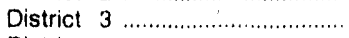 & 567.2 & 66.00 & 22.9 & 82.46 & 590.1 & 74.30 \\
\hline 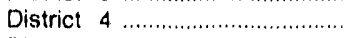 & 460.4 & 60.25 & 354.2 & 86.69 & 814.6 & 84.30 \\
\hline 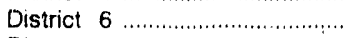 & $w$ & $w$ & $w$ & $w$ & $w$ & $w$ \\
\hline District $7 \ldots \ldots \ldots$ & 194.5 & 63.57 & 2.6 & 75.86 & 197.2 & 64.98 \\
\hline 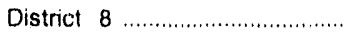 & $1,560.8$ & 63.36 & 782.5 & 81.59 & $2,343.3$ & 68.88 \\
\hline District 9 & 396.7 & 48.33 & 183.3 & 87.03 & 580.1 & 72.05 \\
\hline 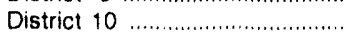 & $1,225.4$ & 46.97 & 155.3 & 78.13 & $1,380.7$ & 57.59 \\
\hline District $11 \ldots \ldots \ldots \ldots \ldots \ldots \ldots \ldots \ldots$ & 78.0 & 53.00 & 396.9 & 82.56 & 474.9 & 79.48 \\
\hline District 12 & - & - & w & $w$ & $w$ & $w$ \\
\hline District $13 \ldots \ldots \ldots \ldots \ldots \ldots \ldots \ldots$ & 364.1 & 60.31 & 155.8 & 86.68 & 519.9 & 78.32 \\
\hline 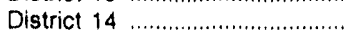 & - & - & w & $w$ & w & $w$ \\
\hline District 15 & $w$ & $w$ & $w$ & $w$ & $1,448.7$ & 83.24 \\
\hline 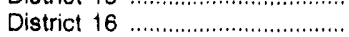 & - & - & $w$ & $w$ & W & $\frac{0.44}{w}$ \\
\hline District 17 & 492.8 & 62.40 & 184.0 & 87.17 & 676.9 & 69.48 \\
\hline District 18 & - & - & $1,889.4$ & 91.00 & $1,889.4$ & 91.00 \\
\hline District 19 & w & w & W & $w$ & $6,287.7$ & 87.18 \\
\hline District 20 & 518.6 & 56.16 & - & - & 518.6 & 56.16 \\
\hline District 21 & - & .. & $1,360.6$ & 89.30 & $1,360.6$ & 89.30 \\
\hline 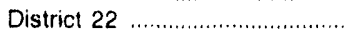 & - & - & $1,513.7$ & 91.00 & $1,513.7$ & 91.00 \\
\hline 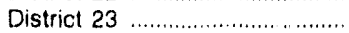 & - & - & w & $w$ & w & 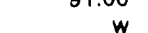 \\
\hline 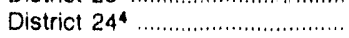 & 34.1 & 71.25 & 26.0 & 61.38 & 60.1 & 63.51 \\
\hline District $1-23^{4}$ Total .................... & $7,358.4$ & 62.27 & $15,261.3$ & 83.90 & $22,619.7$ & 73.15 \\
\hline U.S. Total .... & $7,392.5$ & 62.34 & $15,287.3$ & 83.22 & $22,679.8$ & 72.96 \\
\hline
\end{tabular}

1 Represents the quantity of coal that can be recovered (i.e. mined) from existing coal reserves at reporting mines.

2 Represents the percentage of coal that can be recovered from coal reserves at reporting mines, averaged for all mines in the reported geographic area.

3 Excludes silt, culm, refuse bank, slurry dam, and dredge operations. Excludes mines producing less than 10,000 short tons of coal during the year.

4 District 24 is the anthracite-producing district in Pennsylvania. Districts $1-23$ represent the total U.S. production of bituminous, subbituminous coal, and ligrite. See Appendix C

withheld to avoid disclosure of individual company data.

Note: Total may not equal sum of components because of independent rounding

Source: Energy Information Adininistration, Form EIA-7A "Coal Production Report." 
Table 41. Recoverable Coal Reserves ${ }^{1}$ at U.S. Mines ${ }^{2}$ by State and Type of Mining, 1988, 1989 (Million Short Tons)

\begin{tabular}{|c|c|c|c|c|c|c|c|c|c|}
\hline \multirow[b]{2}{*}{$\begin{array}{c}\text { Coal-Producing } \\
\text { State }\end{array}$} & \multicolumn{3}{|c|}{ Type of Mining } & & \multicolumn{2}{|c|}{ Total } & \multicolumn{3}{|c|}{1988} \\
\hline & $\begin{array}{l}\text { Undergr } \\
\text { Recoverable } \\
\text { Reserves }\end{array}$ & $\begin{array}{l}\text { ound } \\
\text { Percent } \\
\text { Change } \\
\text { from } \\
\text { Previous } \\
\text { Year }\end{array}$ & $\begin{array}{c}\text { Recoverable } \\
\text { Reserves }\end{array}$ & $\begin{array}{l}\text { Percent } \\
\text { Change } \\
\text { from } \\
\text { Prevlous } \\
\text { Year }\end{array}$ & $\begin{array}{c}\text { Aecoverable } \\
\text { Reserves }\end{array}$ & $\begin{array}{l}\text { Percent } \\
\text { Change } \\
\text { from } \\
\text { Previous } \\
\text { Year }\end{array}$ & Underground & Surface & Total \\
\hline Alabama & 359.2 & 4.51 & 148.6 & -11.01 & 507.7 & -0.56 & 343.6 & 166.9 & 510.6 \\
\hline 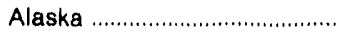 & - & - & w & w & $w$ & $w$ & - & w & w \\
\hline 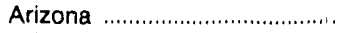 & - & - & $w$ & $w$ & $w$ & $w$ & - & $w$ & $w$ \\
\hline 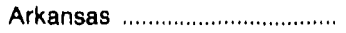 & - & - & $w$ & $w$ & $w$ & $w$ & - & $w$ & $w$ \\
\hline 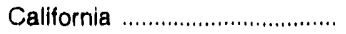 & - & - & $w$ & $w$ & $w$ & w & - & $w$ & $w$ \\
\hline 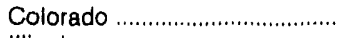 & 476.9 & 6.36 & 185.1 & -9.12 & 662.1 & 1.52 & 448.4 & 203.7 & 652.1 \\
\hline 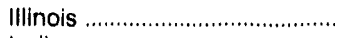 & $1,225.4$ & .81 & 155.3 & 15.07 & $1,380.7$ & 2.24 & $1,215.5$ & 135.0 & $1,350.5$ \\
\hline 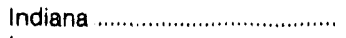 & 78.0 & 25.35 & 396,9 & .98 & 474.9 & 4.31 & 62.2 & 393.1 & 455.3 \\
\hline lowa & - & - & $w$ & $w$ & $w$ & w & - & w & $w$ \\
\hline 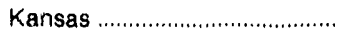 & - & - & 27.6 & w & 27.6 & $w$ & - & $w$ & $w$ \\
\hline 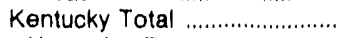 & $1,012.0$ & -10.62 & 501.2 & -16.01 & $1,513.2$ & -12.48 & $1,132.2$ & 596.8 & $1,729.0$ \\
\hline Kentucky, Eastern .................. & 615.3 & -17.63 & 317.8 & -15.27 & 933.1 & -16.84 & 747.0 & 375.2 & $1,122.1$ \\
\hline Kentucky, Western ................ & 396.7 & 2.97 & 183.3 & -17.27 & 580.1 & -4.42 & 385.3 & 221.6 & 606.9 \\
\hline 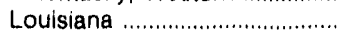 & - & - & $w$ & w & $w$ & $w$ & - & $w$ & $w$ \\
\hline 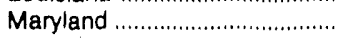 & $w$ & $w$ & $w$ & $w$ & 86.7 & -2.61 & $w$ & $w$ & 89.0 \\
\hline 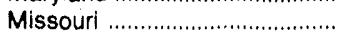 & - & - & $w$ & $w$ & w & w & - & 131.1 & 131.1 \\
\hline 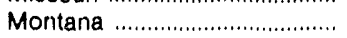 & - & - & $1,513.7$ & -3.76 & $1,513.7$ & -3.76 & - & $1,572.9$ & $1,572.9$ \\
\hline 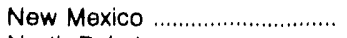 & $w$ & $w$ & w & $w$ & $1,410.7$ & -11.29 & $w$ & $w$ & $1,590.2$ \\
\hline 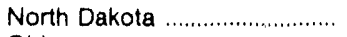 & - & - & $1,360.6$ & -2.33 & $1,360.6$ & -2.33 & - & $1,393.1$ & $1,393.1$ \\
\hline Ohio & 460.4 & 11.01 & 354.2 & -11.10 & 814.6 & .17 & 414.7 & 398.4 & 813.2 \\
\hline 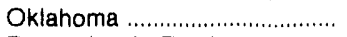 & w & - & $w$ & w & 23.7 & -33.17 & - & 35.5 & 35.5 \\
\hline Pennsyivania Total ................... & 824.5 & -17.15 & 359.6 & 25.91 & $1,184.1$ & -7.55 & 995.2 & 285.6 & $1,280.8$ \\
\hline 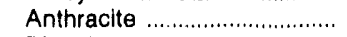 & 34.1 & 10.39 & 26.0 & -3.44 & 60.1 & 3.96 & 30.9 & 26.9 & 57.8 \\
\hline 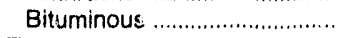 & 790.4 & -18.03 & 333.6 & 28.96 & $1,124.0$ & -8.09 & 964.3 & 258.7 & $1,223,0$ \\
\hline 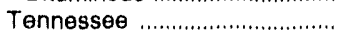 & $w$ & w & W & $w$ & 71.6 & -35.96 & $w$ & w & 111.8 \\
\hline 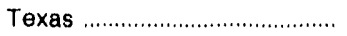 & - & - & $1,135.3$ & -2.73 & $1,135.3$ & -2.73 & - & $1,167.2$ & $1,167.2$ \\
\hline 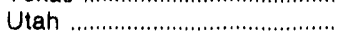 & 518.6 & -3.49 & - & - & 518.6 & -3.49 & 537.4 & - & 537.4 \\
\hline 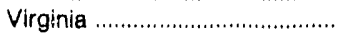 & 353.5 & 2.54 & 66.2 & -.57 & 419.7 & 2.03 & 344.7 & 66.6 & 411.3 \\
\hline 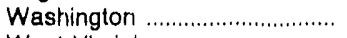 & - & - & $w$ & w & w & $w$ & - & w & w \\
\hline 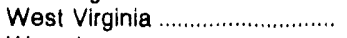 & $1,889.7$ & -4.95 & 423.7 & 11.58 & $2,313,4$ & -2.30 & $1,988.1$ & 379.7 & $2,367,8$ \\
\hline 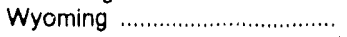 & w & $w$ & $w$ & $w$ & $6,287.7$ & -2.45 & $w$ & $w$ & $6,445.9$ \\
\hline 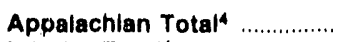 & $w$ & $w$ & $w$ & $w$ & $6,330.9$ & -.5 .60 & $w$ & $w$ & $6,706.6$ \\
\hline Interior Total ${ }^{4}$ & $w$ & w & $w$ & $w$ & $3,906.6$ & -1.81 & $w$ & $\mathbf{w}$ & $3,978.6$ \\
\hline Western Tota| $\left.\right|^{4} \ldots \ldots \ldots \ldots \ldots \ldots \ldots$ & $1,092.1$ & 5.14 & $11,350.2$ & -4.27 & $12,442.3$ & -3.51 & $1,038.7$ & $11,856.7$ & $12,895.4$ \\
\hline $\begin{array}{l}\text { East of the Miss. Rlver ........ } \\
\text { West of the Miss. Plver ....... }\end{array}$ & $\begin{array}{l}6,300.2 \\
1,092.3\end{array}$ & $\begin{array}{r}-5.00 \\
5.17\end{array}$ & $\begin{array}{r}2,466.4 \\
12,820.9\end{array}$ & $\begin{array}{r}-.86 \\
-4.48\end{array}$ & $\begin{array}{r}8,766.6 \\
13,913.2\end{array}$ & $\begin{array}{l}-3.87 \\
-3.79\end{array}$ & $\begin{array}{l}6,631.5 \\
1,038.7\end{array}$ & $\begin{array}{r}2,487.7 \\
13,422.6\end{array}$ & $\begin{array}{r}9,119.3 \\
14,461.3\end{array}$ \\
\hline 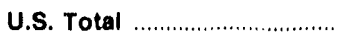 & $7,392.5$ & -3.62 & $15,287.3$ & -3.92 & $22,679.8$ & -3.82 & $7,670.2$ & $15,910.3$ & $23,580.6$ \\
\hline
\end{tabular}

1 Represents the quantity of coal that can be recovered (i.e. mined) from existing coal reserves at reporting mines.

2 Excludes silt, culm, refuse bank, slurry dam, and dredge operations. Excludes mines producing less than 10,000 short tons of coal during the year.

3 Percent changes are calculated using unrounded data.

4 For a definition of coal-producing regions, see Appendix C.

- Withheld to avoid disclosure of individual company data.

Note: Total may not equal sum of components because of independent rounding.

Source: Energy Information Adrninistration, Form ElA-7A, "Coal Production Report." 
Table 42. Recoverable Coal Reserves ${ }^{1}$ at U.S. Mines ${ }^{2}$ by District and Type of Mining, 1988, 1989

(Million Short Tons)

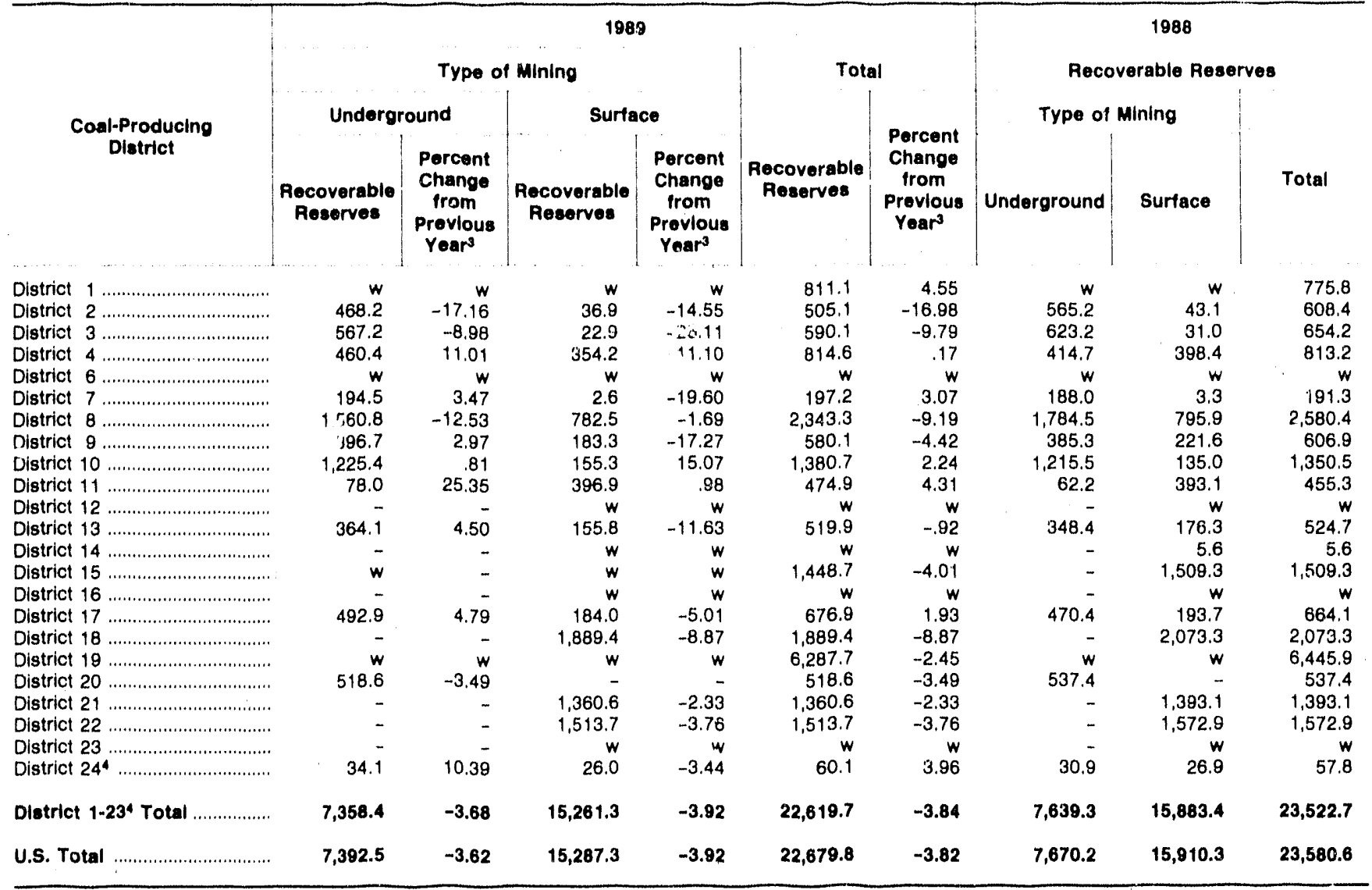

1 Represents the quantity of coal that can be recovered (i.e. mined) from existing coal reserves at reporting mines.

2 Excludes silt, culm, refuse bank, slurry dam, and dredge operations. Excludes mines producing less than 10,000 short tons of coal during the year.

3 Percent changes are calculated using unrounded data.

- District 24 is the anthracite-producing district in Pennsylvania. Districts 1-23 represent the total U.S. production of bituminous, subbituminous coal, and lignite. See A.ppendix $\mathrm{C}$.

Withheld to avoid disclosure of individual company data.

Note: Total may not equal sum of components because of independent rounding.

Source: Energy Information Administration, Form EIA-7A, "Coal Production Report." 
Table 43. Recoverable Coal Reserves ${ }^{1}$ at U.S. Mines ${ }^{2}$ by Mine Production Range and Type of Mining, 1988, 1989

(Million Short Tons)

\begin{tabular}{|c|c|c|c|c|c|c|c|c|c|}
\hline \multirow{4}{*}{$\begin{array}{l}\text { Mine Production Range } \\
\text { (Thousand Short Tons) }\end{array}$} & \multicolumn{6}{|c|}{1989} & \multicolumn{3}{|c|}{1988} \\
\hline & \multicolumn{4}{|c|}{ Type of Mining } & \multicolumn{2}{|c|}{ Total } & \multicolumn{3}{|c|}{ Recoverable Reserves } \\
\hline & \multicolumn{2}{|c|}{ Underground } & \multicolumn{2}{|c|}{ Surface } & \multirow[b]{2}{*}{$\begin{array}{c}\text { Recoverable } \\
\text { Reserves }\end{array}$} & \multirow[b]{2}{*}{$\begin{array}{c}\text { Percent } \\
\text { Change } \\
\text { from } \\
\text { Previoua } \\
\text { Year }\end{array}$} & \multicolumn{2}{|c|}{ Type of Mining } & \multirow[b]{2}{*}{ Total } \\
\hline & $\begin{array}{c}\text { Aecoverable } \\
\text { Aeserves }\end{array}$ & $\begin{array}{c}\text { Percent } \\
\text { Change } \\
\text { from } \\
\text { Previous } \\
\text { Year }\end{array}$ & $\begin{array}{c}\text { Recoverable } \\
\text { Reserves }\end{array}$ & $\begin{array}{l}\text { Percent } \\
\text { Change } \\
\text { from } \\
\text { Prevlous } \\
\text { Year }{ }^{3}\end{array}$ & & & Underground & Surface & \\
\hline 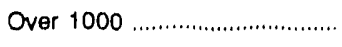 & $4,512.0$ & 2.33 & $12,899.8$ & -2.70 & $17,411.8$ & -1.44 & $4,409.3$ & $13,257,2$ & $17,666.5$ \\
\hline 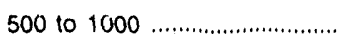 & $1,313.4$ & -1.40 & 563.8 & -52.34 & $1,877.3$ & -25.36 & 1,332.1 & 1.183 .1 & $2,515.2$ \\
\hline 200 to $500 \ldots \ldots$ & 639.8 & -28.04 & 568.6 & -15.73 & $1,208.3$ & -22.73 & 889.1 & 674.7 & $1,563.8$ \\
\hline 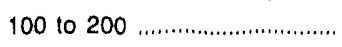 & 434.4 & -29.14 & 503.9 & 86.10 & 938.3 & 6.16 & 613.0 & 270.8 & 883.8 \\
\hline 50 to $100 \ldots \ldots$ & 188.5 & -16.03 & 200.7 & -36.59 & 389.2 & -28.06 & 224.5 & 316.5 & 541.0 \\
\hline 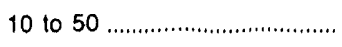 & 304.5 & 50.59 & 550.5 & 164.60 & 855.0 & 108.40 & 202.2 & 208.0 & 410.2 \\
\hline 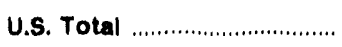 & $7,392.5$ & -3.62 & $15,287.3$ & -3.02 & $22,679.8$ & -3.82 & $7,670.2$ & $15,910.3$ & $23,580.6$ \\
\hline
\end{tabular}

1 Represents the quantity of coal that can be recovered (l.e. mined) from existing coal reserves at reporting mines.

2 Excludes silt, culm, refuse bank, slurry dam, and dredge operations. Excludes mines producing less than 10,000 short tons of coal during the year.

3 Percent changes are calculated using unrounded data.

Note: Total may not equal sum of components because of independent rounding.

Source: Energy Intormation Administration, Form EIA-7A, "Coal Production Report." 


\title{
Appendix A
}

\author{
Demonstrated \\ Reserve Base
}

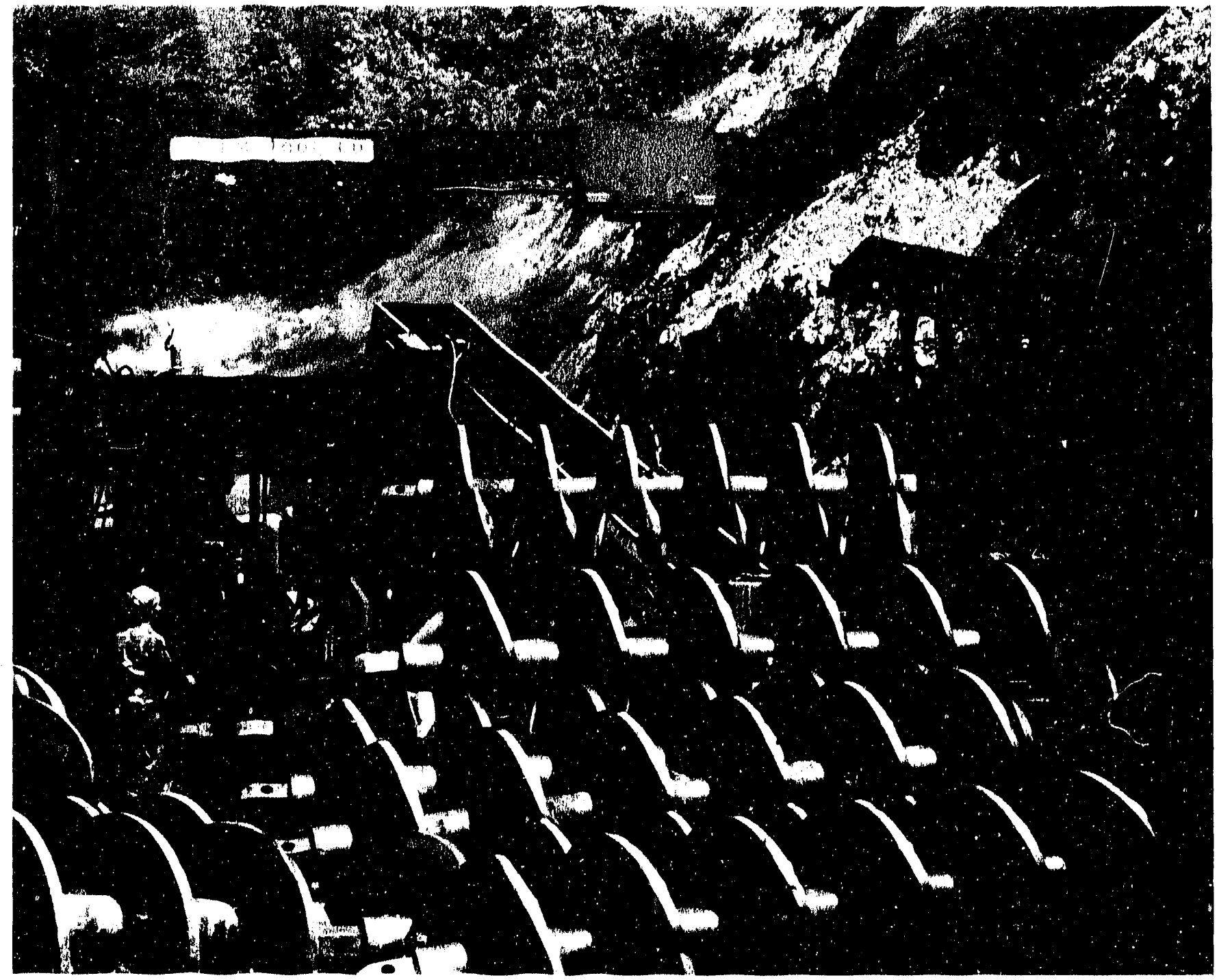

Auger mining is used when the overburden is too costly to excavate. These large-diameter drills are being driven into a coalbed in the side of a stripped pit. 


\section{Appendix A}

\section{Demonstrated Reserve Base}

EIA has calculated a demonstrated reserve base (DRB) of coal in the United States as of January 1, 1990, that contains an estimated 472 billion short tons. At least 50 percent of the DRB total is considered to be recoverable with today's technologies. However, neither the DRB nor the recoverable portion of the DRB is meant to represent the precise amount of coal that can be profitably mined, given current laws, regulations, economics, and usages. Depletion adjustments for the calendar year were made using 1989 production data.

Widely distributed geographically, over half of the 1990 DRB occurs in States West of the Mississippi River (Table A1). Almost a third of the total DRB is presently considered attainable by surface mining, and nearly three-quarters of that surface-minable coal is located West of the Mississippi. Conversely, over half of the WRB coal currently requiring underground mining is located in States East of the Mississippi.

Coal ranks are also distributed differently among coal regions. About half of the DRB is bituminous coal, which is concentrated primarily in the States East of the Mississippi. Subbituminous coal accounts for 38 percent of the DRB and is all located West of the Mississippi, primarily in the Rocky Mountain and Pacific Northwest States. Nearly 10 percent of the DRB is lignite, almost all of which is located in the West. Only 1.6 percent of the DRB is anthracite, which is concentrated almost entirely in northeastern Pennsylvania.

Detailed data by State, coal rank, and potential method of mining are presented in Tables A1 through A4. All tables include the previous DRB estimates (as of January 1,1989$)$ for comparison. No new data sources or adjustments based on changes in DRB criteria or data analysis were used in the 1990 DRB. The differences between 1989 and 1990 data are based on depletion adjustments using production in 1989.

The Explanatory Notes section (Appendix D) includes a summary of all adjustments, except for normal annual depletion adjustments, performed on DRB data since EIA took over DRB responsibilities in 1977. Also described there are plans for a new program to develop improved DRB and reserves data. 
Table A1. Demonstrated Reserve Base of Coal by Rank (Million Short Tons)

\begin{tabular}{|c|c|c|c|c|c|c|c|c|c|c|}
\hline \multirow{2}{*}{$\begin{array}{l}\text { Coal Producing Region } \\
\text { and State }\end{array}$} & \multicolumn{2}{|c|}{ Anthracite } & \multicolumn{2}{|c|}{ Bltuminous } & \multicolumn{2}{|c|}{ Subbituminous } & \multicolumn{2}{|c|}{ Lignite } & \multicolumn{2}{|c|}{ Total } \\
\hline & 1989 & 1990 & 1989 & 1990 & 1989 & 1990 & 1989 & 1980 & 1989 & 1990 \\
\hline Alabama & - & - & $3,822.0$ & $3,774.8$ & - & - & $1,083.0$ & $1,083.0$ & $4,905.0$ & $4,857.8$ \\
\hline 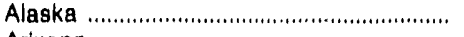 & - & - & 697.5 & 697.5 & $5,430.2$ & $5,428.2$ & 14.0 & 14.0 & $6,141.7$ & $6,139.7$ \\
\hline 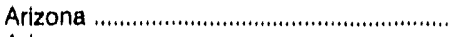 & - & - & 281.6 & 266.6 & - & - & - & - & 281.6 & 266.6 \\
\hline 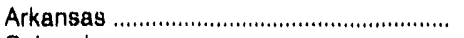 & 104.1 & 104.1 & 287.7 & 287.6 & - & - & 25.4 & 25.4 & 417.1 & 417.0 \\
\hline 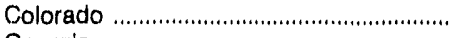 & 25.5 & 25.5 & $8,923.0$ & $8,803,4$ & $3,906.8$ & $3,898,7$ & $4,189.9$ & $4,189.9$ & $17,045.2$ & $17,017,4$ \\
\hline 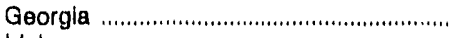 & - & - & 3.2 & 3.2 & - & - & - & - & 3.2 & 3.2 \\
\hline 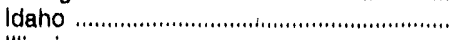 & - & - & 4.4 & 4.4 & - & - & - & - & 4.4 & 4.4 \\
\hline 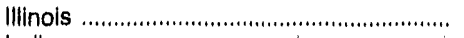 & - & - & $78,434,9$ & $78,331.3$ & - & - & - & - & $78,434,9$ & $78,331.3$ \\
\hline 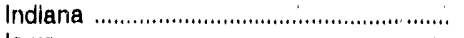 & - & - & $10,243.1$ & $10,199.2$ & - & - & - & - & $10,243.1$ & $10,199.2$ \\
\hline 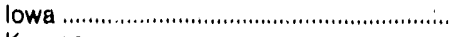 & - & - & $2,191,6$ & $2,191,0$ & - & - & - & - & $2,191.6$ & $2,191.0$ \\
\hline 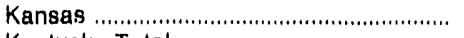 & - & - & 979.3 & 978.3 & - & - & - & - & 979.3 & 978.3 \\
\hline 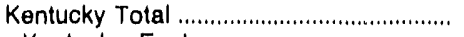 & - & - & $29,926.5$ & $29,643,0$ & - & - & - & - & $29,926,5$ & $29,643.0$ \\
\hline Kertucky, Eastern .................................... & - & - & $9,439.1$ & $9,225.1$ & - & - & .. & - & $9,439.1$ & $9,225.1$ \\
\hline 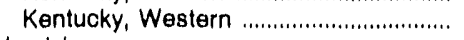 & - & - & $20,487,4$ & $20,417.9$ & - & - & - & - & $20,487.4$ & $20,417.9$ \\
\hline 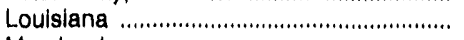 & - & - & - & - & - & - & 495.3 & 491.6 & 495.3 & 491.6 \\
\hline 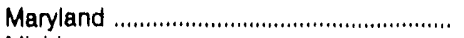 & - & - & 768.0 & 762.4 & - & - & - & - & 768,0 & 762.4 \\
\hline Michigan & + & - & 127.7 & 127.7 & - & - & - & - & 127.7 & 127.7 \\
\hline 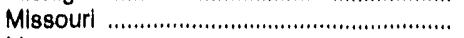 & - & - & $6,011.5$ & $6,007.3$ & - & - & - & - & $6,011.5$ & $6,007.3$ \\
\hline 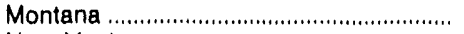 & - & - & $1,385.4$ & $1,385.4$ & $102,812.7$ & $102,865.8$ & $15,762.6$ & $15,762.2$ & $120,060.7$ & $120,013.5$ \\
\hline 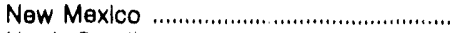 & 2.3 & 2.3 & $1,977.4$ & $1,961.8$ & $2,537.2$ & $2,523.2$ & - & - & $4,516.9$ & $4,487.2$ \\
\hline North Carolina & - & - & 10.7 & 10.7 & - & - & - & - & 10.7 & 10.7 \\
\hline 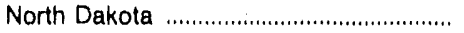 & - & - & - & - & - & - & $9,700.4$ & $9,663,4$ & $9,700.4$ & $9,663.4$ \\
\hline 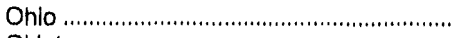 & - & - & $18,565.5$ & $18,515.3$ & - & - & - & - & $18,565.5$ & $18,515.3$ \\
\hline 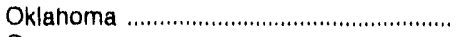 & - & - & $1,593.3$ & $1,591.1$ & - & - & - & - & $1,593.3$ & $1,591.1$ \\
\hline Oregon & - & - & - & - & 17.5 & 17.5 & - & - & 17.5 & 17.5 \\
\hline 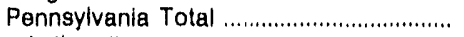 & $7,061.0$ & $7,057.7$ & $22,290.5$ & $22,177.1$ & - & - & - & - & $29,351.4$ & $29,234.8$ \\
\hline 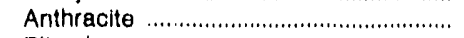 & $7,061.0$ & $7,057.7$ & - & - & .. & .- & - & - & $7,061.0$ & $7,057.7$ \\
\hline 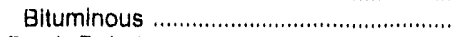 & - & - & $22,290,5$ & $22,177.1$ & - & - & - & - & $22,290.5$ & $22,177.1$ \\
\hline 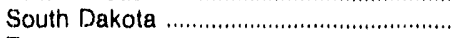 & - & - & - & - & - & - & 366.1 & 366.1 & 366.1 & 366.1 \\
\hline 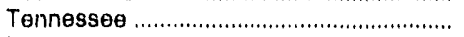 & - & - & 867.5 & 855.9 & - & - & - & - & 867.5 & 855.9 \\
\hline Texas & - & - & - & - & - & - & $13,469.0$ & $13,402.1$ & $13,469.0$ & $13,402.1$ \\
\hline Utah & - & - & $6,216.9$ & $6,176.7$ & 1.1 & 1.1 & - & - & $6,218.0$ & $6,177.8$ \\
\hline 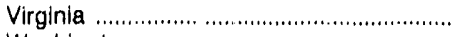 & 125.5 & 125.5 & $2,667.2$ & $2,586.4$ & - & - & - & - & $2,792.6$ & $2,711.9$ \\
\hline 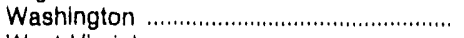 & - & - & 303.7 & 303.7 & $1,119.2$ & 1.113 .1 & 8.1 & 8.1 & $1,431.0$ & $1,424.8$ \\
\hline 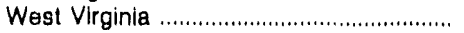 & - & - & $37,666.1$ & $37,389.2$ & - & - & - & - & $37,666.1$ & $37,389.2$ \\
\hline 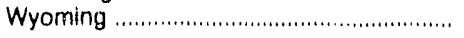 & - & - & $4,412.6$ & $4,408.8$ & $64,041.0$ & $63,829.1$ & - & - & $68,453.6$ & $68,237,8$ \\
\hline 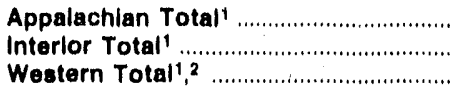 & $\begin{array}{r}7,186.5 \\
104.1 \\
27.8\end{array}$ & $\begin{array}{r}7,183.2 \\
104.1 \\
27.8\end{array}$ & $\begin{array}{r}96,099.8 \\
120,356.5 \\
24,202.5\end{array}$ & $\begin{array}{r}95,300.1 \\
12 C, 131.3 \\
24,108.3\end{array}$ & $179,965 . \overline{-}$ & $179,676 . \overline{5}$ & $\begin{array}{r}1,083.0 \\
13,989.7 \\
30,041.0\end{array}$ & $\begin{array}{r}1,083.0 \\
13,919.0 \\
30,003.7\end{array}$ & $\begin{array}{l}104,369.3 \\
134,450.2 \\
234,236.8\end{array}$ & $\begin{array}{l}103,566.4 \\
134,154.3 \\
233,816.3\end{array}$ \\
\hline 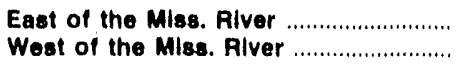 & $\begin{array}{r}7,186.5 \\
131.9\end{array}$ & $\begin{array}{r}7,183.2 \\
131.9\end{array}$ & $\begin{array}{r}205,392.9 \\
35,265.9\end{array}$ & $\begin{array}{r}204,376.2 \\
35,163.6\end{array}$ & $179,965 . \overline{5}$ & $179,676 . \overline{5}$ & $\begin{array}{r}1,083.0 \\
44,030.7\end{array}$ & $\begin{array}{r}1,083.0 \\
43,922.6\end{array}$ & $\begin{array}{l}213,662.4 \\
259,394.0\end{array}$ & $\begin{array}{l}212,642.4 \\
258,894.6\end{array}$ \\
\hline 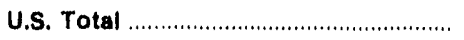 & $7,318.3$ & $7,315.1$ & $240,658.9$ & $239,539.7$ & $179,965.5$ & $179,676.5$ & $45,113.7$ & $45,005.6$ & $473,056.3$ & $471,537.0$ \\
\hline
\end{tabular}

1 For a detinition of coal-producing regions, see Appendix $\mathrm{C}$.

2 The 1989 Western Region totals, as published in Coal Production 1988, did not include the subbituminous data for Oregon or the lignite data for South Dakota.

Note: Total may not equal sum of components because of independent rounding. Data are reported as of the first day of the year.

Source: State geological and mineral resource surveys, and other geological reports. 
Table A2. Demonstrated Reserve Base of Coal by Potential Method of Mining (Million Short Tons)

\begin{tabular}{|c|c|c|c|c|c|c|}
\hline \multirow{2}{*}{$\begin{array}{l}\text { Coal Producing Region } \\
\text { and State }\end{array}$} & \multicolumn{2}{|c|}{ Underground } & \multicolumn{2}{|c|}{ Surface } & \multicolumn{2}{|c|}{ Total } \\
\hline & 1989 & 1990 & $\$ 989$ & 1990 & 1989 & 1990 \\
\hline 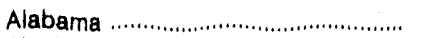 & $1,555.7$ & $1,523.0$ & $3,349.4$ & $3,334,8$ & $4,905.0$ & $4,857,8$ \\
\hline 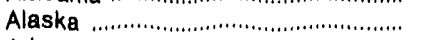 & $5,423,0$ & $5,423.0$ & 718.7 & 716.7 & $6,141.7$ & $6,139,7$ \\
\hline 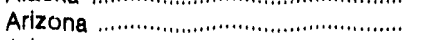 & 101.6 & 101.6 & 180.0 & 165.1 & 281.6 & 266.6 \\
\hline Arkansas .............................................. & 272.5 & 272.5 & 144.6 & 144.5 & 417.1 & 417.0 \\
\hline 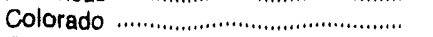 & $12,185,4$ & $12,168.4$ & $4,859.8$ & $4,849.0$ & $17,045.2$ & $17,017.4$ \\
\hline 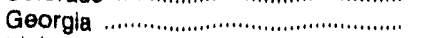 & 1.9 & 1.8 & 1.3 & $1.3^{\prime \prime}$ & 3.2 & 3.2 \\
\hline 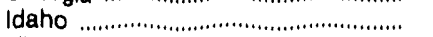 & 4.4 & 4.4 & - & - & 4.4 & 4.4 \\
\hline 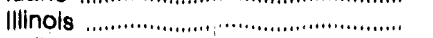 & $62,979.7$ & $62,901.1$ & $15,455.2$ & $15,430.2$ & $78,434.9$ & $78,331.3$ \\
\hline 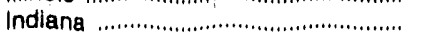 & $8,906.7$ & $8,901.7$ & $1,336.4$ & $1,297.5$ & $10,243.1$ & $10,199.2$ \\
\hline lowa & $1,732.6$ & $1,732.5$ & 459.0 & 458.5 & $2,191.6$ & $2,191.0$ \\
\hline 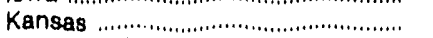 & - & - & 979.3 & 978.3 & 979.3 & 978.3 \\
\hline 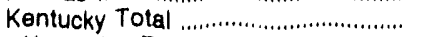 & $24,311.2$ & $24,113.1$ & $5,615.3$ & $5,529,9$ & $2 \theta, 926.5$ & $29,643,0$ \\
\hline Kentucky, Eastern ............................. & $7,675.3$ & 7.523 .8 & $1,763.8$ & $1,701.3$ & $0,439.1$ & $9,225.1$ \\
\hline 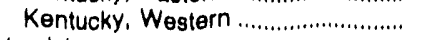 & $16,635.9$ & $16,589.3$ & $3,861.5$ & $3,828.6$ & $20,48 \% .4$ & $20,417.9$ \\
\hline 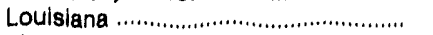 & - & - & 495.3 & 491.6 & 495.3 & 491.6 \\
\hline 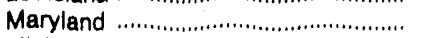 & 677.3 & 673.7 & 90.7 & 88.8 & 768.0 & 762.4 \\
\hline 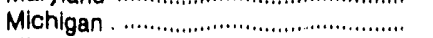 & 123.1 & 123.1 & 4.6 & 4.6 & 127.7 & 127.7 \\
\hline 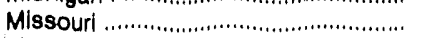 & $1,479.1$ & $1,479.1$ & $4,532.4$ & $4,528.2$ & $6,011.5$ & $6,007,3$ \\
\hline 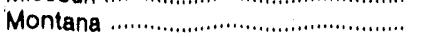 & $70,958.7$ & $70,958.7$ & $49,102.0$ & $49,054.8$ & $120,060.7$ & $120,013,6$ \\
\hline 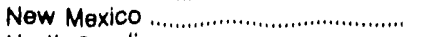 & $2,121,8$ & $2,121.8$ & $2,395.0$ & $2,365.5$ & $4,516.9$ & $4,487,2$ \\
\hline North Carolina ..................................... & 10.7 & 10.7 & - & - & 10.7 & 10.7 \\
\hline 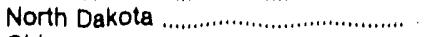 & - & - & $9,700.4$ & $\theta, 663.4$ & $8,700.4$ & $9,663.4$ \\
\hline 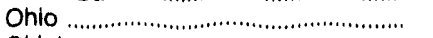 & $12,841.6$ & $12,819.9$ & $5,724.0$ & $5,695.4$ & $18,565.5$ & $18,515.3$ \\
\hline 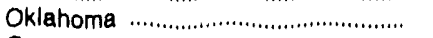 & $1,238.3$ & $1,238.2$ & 355.0 & 352.9 & $1,593.3$ & $1,591.1$ \\
\hline 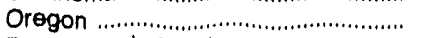 & 14.5 & 14.5 & 2.9 & 2.9 & 17.5 & 17.5 \\
\hline Pennsylvania Total .............................. & $27,993.9$ & $27,914.7$ & $1,357.5$ & $1,320.1$ & $29,351,4$ & $29,234.8$ \\
\hline 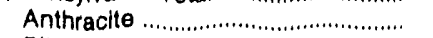 & $6,954,8$ & $6,853.8$ & 106.1 & 103.8 & $7,061.0$ & $7,057.7$ \\
\hline 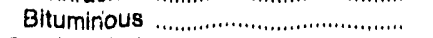 & $21,039.1$ & $20,960.9$ & $1,251.4$ & $1,216.2$ & $22,290.5$ & $22,177.1$ \\
\hline 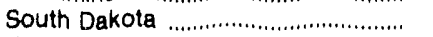 & - & - & 366.1 & 366.1 & 366.1 & 368.1 \\
\hline 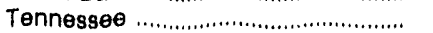 & $568 . \theta$ & 559.5 & 298.6 & 296.3 & 867.5 & 855.8 \\
\hline 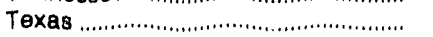 & - & - & $13,469.0$ & $13,402.1$ & $13,469,0$ & $13,402.1$ \\
\hline Utah & $5,950.1$ & $5,909.9$ & 267.9 & 267.8 & $6,218.0$ & $6,177.8$ \\
\hline 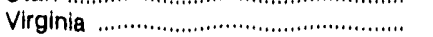 & $2,034.2$ & $1,83 \div 1$ & 758.5 & 749.8 & $2,792.6$ & $2,711,8$ \\
\hline 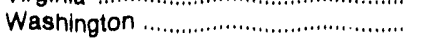 & $1,332.3$ & $1,332.3$ & 98.7 & 92.6 & $1,431.0$ & $1,424.6$ \\
\hline 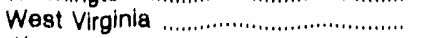 & $32,761,4$ & $32,535.0$ & $4,904.7$ & $4,854.3$ & $37,666.1$ & $37,389.2$ \\
\hline 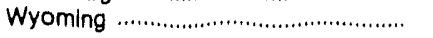 & $42,550.0$ & $42,546.7$ & $25,903.6$ & $25,691.2$ & $68,453.6$ & $68,237,8$ \\
\hline 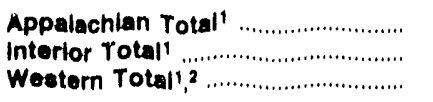 & $\begin{array}{r}86,120.8 \\
93,368.0 \\
140,841.8\end{array}$ & $\begin{array}{r}85,524.2 \\
93,237.5 \\
140,581.2\end{array}$ & $\begin{array}{l}18,248.5 \\
41,082.3 \\
93,595.0\end{array}$ & $\begin{array}{l}18,042.2 \\
40,916.9 \\
93,235.1\end{array}$ & $\begin{array}{l}104,369.3 \\
134,450.2 \\
234,236.8\end{array}$ & $\begin{array}{l}103,566.4 \\
134,154.3 \\
233,816.3\end{array}$ \\
\hline $\begin{array}{l}\text { East of the Mles. Alver .................. } \\
\text { West of the Mise. Rlver ................ }\end{array}$ & $\begin{array}{l}174,766.3 \\
145,364.3\end{array}$ & $\begin{array}{l}174,039.3 \\
115,303.5\end{array}$ & $\begin{array}{r}38,896.1 \\
114,029.7\end{array}$ & $\begin{array}{r}38,603.0 \\
113,591.1\end{array}$ & $\begin{array}{l}213,682.4 \\
259,384.0\end{array}$ & $\begin{array}{l}212,642.4 \\
258,894.6\end{array}$ \\
\hline 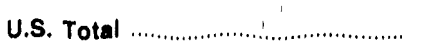 & $320,130.5$ & $319,342.8$ & $152,925.8$ & $152,184.1$ & $473,056.3$ & $471,537.0$ \\
\hline
\end{tabular}

1 For a definition of coal-producing regions, see Appendix C.

2 The 1989 Western Fegion totals, as published in Coal Production 1988, did not include the subbituminous data for Oregon or the lignite data for South Dakota.

Note: Total may not equal sum of components because of independent rounding. Data are reported as of the first day of the year. Source: State geological and mineral resource surveys, and other geological reports. 


\section{Table A3. Demonstrated Reserve Base of Coal by Rank, Potentially Minable by Underground Methods (Million Short Tons)}

\begin{tabular}{|c|c|c|c|c|c|c|c|c|c|c|c|}
\hline \multirow{2}{*}{$\begin{array}{l}\text { Coal Producing Region } \\
\text { and State }\end{array}$} & \multicolumn{2}{|c|}{ Anthracite } & \multicolumn{2}{|c|}{ Bituminous } & \multicolumn{2}{|c|}{ Subbltuminous } & \multicolumn{3}{|c|}{ Lignite } & \multicolumn{2}{|c|}{ Total } \\
\hline & 1989 & 1980 & 1889 & 1990 & 1889 & 1990 & 1989 & & 1890 & 1989 & 1990 \\
\hline Alabama & - & - & $1,555.7$ & $1,523.0$ & - & - & & - & - & $1,555.7$ & $1,523.0$ \\
\hline Alaska & - & - & 617.0 & 617.0 & $4,805.9$ & $4,805.3$ & & - & - & $5,423.0$ & $5,423.0$ \\
\hline Arizona & - & - & 101.6 & 101.6 & - & - & & - & - & 101.6 & 101.6 \\
\hline Arkansas & 88.6 & 88.6 & 183.9 & 183.9 & - & - & & - & - & 272.5 & 272.5 \\
\hline 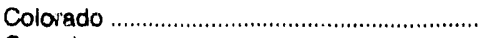 & 25.5 & 25.5 & $8,322.9$ & $8,305.9$ & 3.837 .0 & $3,837.0$ & & - & - & $12,185.4$ & $12,168.4$ \\
\hline Georgia & - & - & 1.9 & 1.9 & - & - & & - & - & 1.9 & 1.9 \\
\hline Idaho & - & - & 4.4 & 4.4 & - & - & & - & - & 4.4 & 4.4 \\
\hline Illinois & - & - & $32,979.7$ & $62,901.1$ & - & - & & - & - & $62,979.7$ & $62,901.1$ \\
\hline 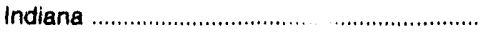 & - & - & $8,906.7$ & $8,901.7$ & - & - & & - & - & $8,906.7$ & $8,901.7$ \\
\hline lowa & - & - & $1,732.6$ & $1,732.5$ & - & - & & - & - & 1.732 .6 & $1,732.5$ \\
\hline 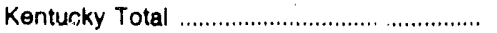 & - & - & $24,311,2$ & $24,113.1$ & - & - & & - & - & $24,311,2$ & $24,113.1$ \\
\hline 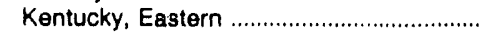 & - & - & $7,675.3$ & $7,523.8$ & - & - & & - & - & $7,675.3$ & $7,523.8$ \\
\hline Kentucky, Westeri & - & - & $16,635.9$ & $16,589.3$ & - & - & & - & - & $16,6^{n=}=9$ & $16,589.3$ \\
\hline Maryland & - & - & 677.3 & 673.7 & - & - & & - & - & 677.3 & 673.7 \\
\hline Michigan & - & - & 123.1 & 123.1 & - & - & & - & - & 123.1 & 123.1 \\
\hline 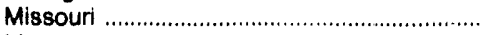 & - & - & $1,479.1$ & $1,479.1$ & -. & - & & - & - & $1,479.1$ & $1,479.1$ \\
\hline Montana & - & - & $1,385.4$ & $1,385,4$ & $69,573.3$ & $69,573.3$ & & - & - & $70,958.7$ & $70,958.7$ \\
\hline 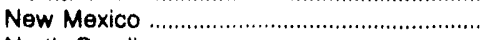 & 2.3 & 2.3 & $1,230.6$ & $1,230.5$ & 889.0 & 889.0 & & - & - & $2,121.9$ & $2,121.8$ \\
\hline North Carolina & - & - & 10.7 & 10.7 & - & - & & - & - & 10.7 & 10.7 \\
\hline Ohio & - & - & $12,841.6$ & $12,819.9$ & - & - & & - & - & $12,841.6$ & $12,819.9$ \\
\hline 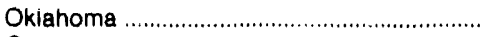 & - & - & $1,238.3$ & $1,238.2$ & - & - & & - & - & $1,238.3$ & $1,238.2$ \\
\hline 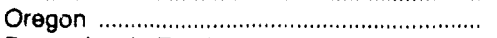 & - & - & - & - & 14.5 & 14.5 & & - & - & $14 . b$ & 14.5 \\
\hline Pennsylvania Total .............................................. & $6,954.8$ & $6,853.8$ & $21,038.1$ & $20,960.9$ & - & - & & - & - & $27,993.9$ & $27,914.7$ \\
\hline 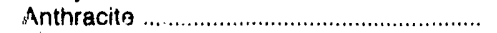 & $6,954.8$ & $6,953.8$ & - & - & - & - & & - & - & $6,954.8$ & $6,953.8$ \\
\hline 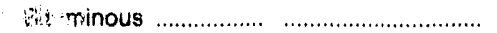 & - & - & $21,039.1$ & $20,960.9$ & - & - & & - & - & $21,039,1$ & $20,960.9$ \\
\hline 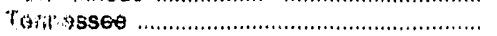 & - & - & 568.9 & 559.5 & - & - & & - & - & 568.9 & 559.5 \\
\hline 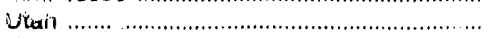 & - & - & $5,949.0$ & $5,908.8$ & 1.1 & 1.1 & & - & - & $5,950.1$ & $5,809.9$ \\
\hline Virginia & 125.5 & 125.5 & $1,908.7$ & $1,836.6$ & - & - & & - & - & $2,034.2$ & $1,962.1$ \\
\hline Weshington & - & - & 303.7 & 303.7 & $1,028.6$ & $1,028.6$ & & - & - & $1,332.3$ & $1,332.3$ \\
\hline 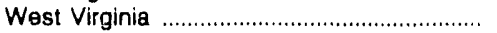 & - & - & $32,761.4$ & $32,535.0$ & - & - & & - & - & $32,761.4$ & $32,535.0$ \\
\hline Wyoming & - & - & $3,887.0$ & $3,883.7$ & $38,663.0$ & $38,660.0$ & & - & - & $42,550.0$ & $42,546.7$ \\
\hline 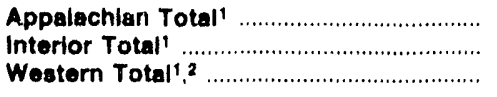 & $\begin{array}{r}7,080.3 \\
88.6 \\
27.6\end{array}$ & $\begin{array}{r}7,079.3 \\
88.6 \\
27.8\end{array}$ & $\begin{array}{l}79,040.5 \\
93,279.4 \\
21,801.6\end{array}$ & $\begin{array}{l}71,44.9 \\
81,48.9 \\
21,761.0\end{array}$ & $118,812 . \overline{-}$ & $118,812 . \overline{4}$ & & $\begin{array}{l}- \\
-\end{array}$ & $\overline{-}$ & $\begin{array}{r}86,120.8 \\
93,368.0 \\
140,641.8\end{array}$ & $\begin{array}{r}85,524.2 \\
93,237.5 \\
140,581.2\end{array}$ \\
\hline 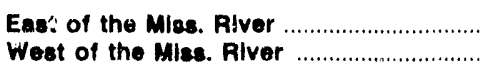 & $\begin{array}{r}7,080.3 \\
116.4\end{array}$ & $\begin{array}{r}7,079.3 \\
116.4\end{array}$ & $\begin{array}{r}167,685.9 \\
26,435.5\end{array}$ & $\begin{array}{r}166,960.1 \\
26,374.7\end{array}$ & $118,812 . \overline{-}$ & $118,812 . \overline{-}$ & & - & - & $\begin{array}{l}174,766.3 \\
145,364.3\end{array}$ & $\begin{array}{l}174,039.3 \\
145,303.5\end{array}$ \\
\hline (1) & $7,196.7$ & $7,195.7$ & $194,121.4$ & $193 ;: 4.8$ & $178,812.4$ & $118,812.4$ & & - & - & $320,130.5$ & $319,342.8$ \\
\hline
\end{tabular}

1 For a definition of coal-producing regions, sea Appendix C.

2 The 1989 Westerr Region totals, as publistied in Coal Production 1988, did not include the subbituminour usta for Oregon or the lignite data for South Dakote.

Note: Total may not equal sum of components because of independent rounding. Data are reported as of the tirst day of the year.

Source: Staie geological and mineral resource surveys, and other geological reports. 


\section{Table A4. Demonstrated Reserve Base of Coal by Rank, Potentially Minable}

by Surface Methods

(Million Short Tons)

\begin{tabular}{|c|c|c|c|c|c|c|c|c|c|c|}
\hline \multirow{2}{*}{$\begin{array}{c}\text { Coal Producing Pegion } \\
\text { and State }\end{array}$} & \multicolumn{2}{|c|}{ Anthracite } & \multicolumn{2}{|c|}{ Bltuminous } & \multicolumn{2}{|c|}{ Subbltuminous } & \multicolumn{2}{|c|}{ Ligr $t$} & \multicolumn{2}{|c|}{ Total } \\
\hline & 1989 & 1990 & 1989 & 1990 & 1989 & 1980 & 1989 & 1800 & 1889 & 1990 \\
\hline Alabama & - & - & $2,266.4$ & $2,251.8$ & - & - & $1,083.0$ & $1,083.0$ & $3,349.4$ & $3,334.8$ \\
\hline Alaska & - & - & 80.5 & 80.5 & 624.2 & 622.2 & 14.0 & 14.0 & 718.7 & 716.7 \\
\hline Arizona & - & - & 180.0 & 165.1 & - & - & - & - & 180.0 & 165.1 \\
\hline Arkansas & 15.5 & 15.5 & 103.8 & 103.7 & - & - & 25.4 & 25.4 & 144.6 & 144.5 \\
\hline 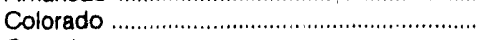 & - & - & 600.1 & 597.5 & 69.8 & 61.7 & $4,189.9$ & $4,189.9$ & $4,859.8$ & $4,849.0$ \\
\hline 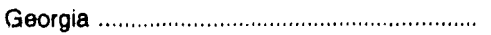 & - & - & 1.3 & 1.3 & - & - & - & - & 1.3 & 1.3 \\
\hline 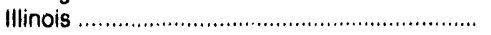 & - & - & $15,455.2$ & $15,430.2$ & - & - & - & - & $15,455.2$ & $15,430.2$ \\
\hline Indiana & - & - & $1,336.4$ & $1,297.5$ & - & - & - & - & $1,336.4$ & $1,297.5$ \\
\hline 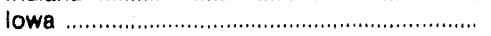 & - & - & 459.0 & 458.5 & - & - & - & - & 459.0 & 458.5 \\
\hline Kansas & - & - & 979.3 & 978.3 & - & - & - & - & 979.3 & 978.3 \\
\hline 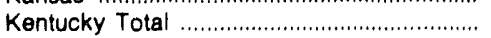 & - & - & $5,615.3$ & $5,529.9$ & - & - & - & - & $5,615.3$ & $5,529.9$ \\
\hline 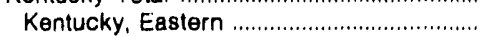 & - & - & $1,763.8$ & $1,701.3$ & - & - & - & - & $1,763.8$ & $1,701.3$ \\
\hline 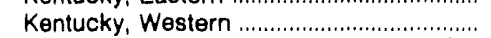 & - & - & $3,851.5$ & $3,828.6$ & - & - & - & - & $3,851.5$ & $3,828.6$ \\
\hline Louisiana & - & - & - & - & 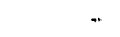 & - & 495.3 & 481.6 & 495.3 & 491.6 \\
\hline 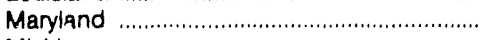 & - & - & 90.7 & 88.8 & - & - & - & - & 90.7 & 88.8 \\
\hline Michigan & - & - & 4.6 & 4.6 & - & - & - & - & 4.6 & 4.6 \\
\hline 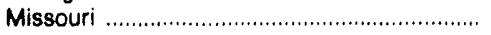 & - & - & 4.532 .4 & $4,528.2$ & - & - & - & - & $4,532.4$ & $4,528.2$ \\
\hline 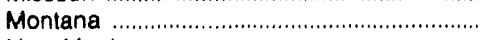 & - & - & - & - & $33,338.4$ & $33,292.6$ & $15,762.6$ & $15,762.2$ & $49,102.0$ & $49,054.8$ \\
\hline 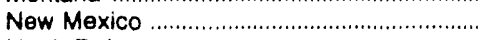 & - & - & 746.9 & 731.3 & $1,648.2$ & $1,634.2$ & - & - & $2,395.0$ & $2,365.5$ \\
\hline North Dakota & - & - & - & - & - & - & $9,700.4$ & $9,663.4$ & $9,700.4$ & $9,663.4$ \\
\hline Ohio & & - & $5,724.0$ & $5,695.4$ & - & - & - & - & $5,724.0$ & $5,695.4$ \\
\hline Oklahoma & - & - & 355.0 & 352.9 & - & - & - & - & 355.0 & 352.9 \\
\hline Oregon & - & - & - & - & 2.9 & 2.8 & - & - & 2.9 & 2.8 \\
\hline 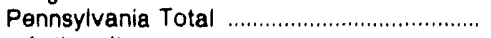 & 106.1 & 103.9 & $1,251.4$ & $1,216.2$ & - & - & - & - & $1,357.5$ & $1,320.1$ \\
\hline Anthracite & 106.1 & 103.9 & - & - & - & - & - & - & 106.1 & 103.9 \\
\hline 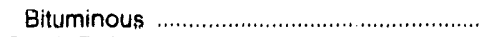 & - & - & $1,251.4$ & $1,216.2$ & - & - & - & - & $1,251.4$ & $1,216.2$ \\
\hline South Dakota & - & -. & - & - & - & - & 366.1 & 366.1 & 366.1 & 366.1 \\
\hline 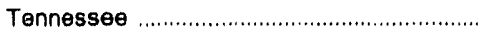 & - & - & 298.6 & 296.3 & - & - & - & - & 298.6 & 296.3 \\
\hline 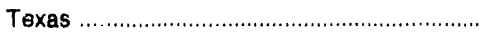 & - & - & - & - & - & - & $13,469.0$ & $13,402.1$ & $13,469.0$ & $13,402.1$ \\
\hline 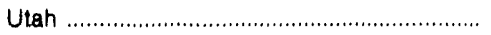 & - & - & 267.9 & 267.9 & - & - & - & - & 267.8 & 267.9 \\
\hline Virginia & - & - & 758.5 & 749.8 & - & - & - & - & 758.5 & 749.8 \\
\hline Washington & - & - & - & - & 90.6 & 84.5 & 8.1 & 8.1 & 98.7 & 92.6 \\
\hline West Virginia & - & - & $4,904.7$ & $4,854.3$ & - & - & - & 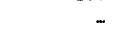 & $4,804.7$ & $4,854.3$ \\
\hline Wyoming & - & - & 525.6 & 525.1 & $25,378.0$ & $25,166.1$ & - & - & $25,903.6$ & $25,691.2$ \\
\hline Appalachlan Total' ${ }^{1}$. & 106.1 & 103.8 & $17,058.4$ & $16,855.2$ & - & - & $1,083.0$ & $1,083.0$ & $18,248.5$ & $18,042.2$ \\
\hline Interlor Total' & 15.5 & 15.5 & $27,077.1$ & $26,982.4$ & - & - & $13,889.7$ & $13,918.0$ & $41,082.3$ & $40,916,9$ \\
\hline 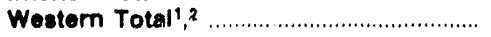 & - & - & $2,400.0$ & $2,367.3$ & $61,153.1$ & $60,864.2$ & $30,041.0$ & $30,003.7$ & $93,595.0$ & $83,235.1$ \\
\hline $\begin{array}{l}\text { East of the Miss. River } \\
\text { West of the Mise. Plver } \ldots \ldots \ldots \ldots \ldots \ldots \ldots \ldots \ldots \ldots \ldots \ldots \ldots \ldots \ldots \ldots\end{array}$ & $\begin{array}{r}106.1 \\
15.5\end{array}$ & $\begin{array}{r}103.9 \\
15.5\end{array}$ & $\begin{array}{r}57,707.0 \\
8,830.5\end{array}$ & $\begin{array}{r}37,416.1 \\
8,788.9\end{array}$ & $61,153 . \overline{1}$ & $60,864 . \overline{2}$ & $\begin{array}{r}1,083.0 \\
44,030.7\end{array}$ & $\begin{array}{r}1,083.0 \\
43,922.6\end{array}$ & $\begin{array}{r}38,896.1 \\
114,029.7\end{array}$ & $\begin{array}{r}38,603.0 \\
113,591.1\end{array}$ \\
\hline (1) & 121.6 & 119.4 & $46,537.5$ & $46,205.0$ & $61,153.1$ & $60,864.2$ & $45,113.7$ & $45,005.6$ & $152,825.8$ & $152,194.1$ \\
\hline
\end{tabular}

1 For a definition of coal.producing regions, see Appendix C.

2 The 1989 Western Region totals, as published in Coal/ Production 1988, did not include the subbituminous data for Oregen or the lignite data for South Dakoia.

Note: Total may not equal sum of components because of independent rounding. Data are reported as of the first day of the year.

Source: State geological and mineral resource surveys, and other geological reports. 


\section{Appendix B}

State Coal Facts

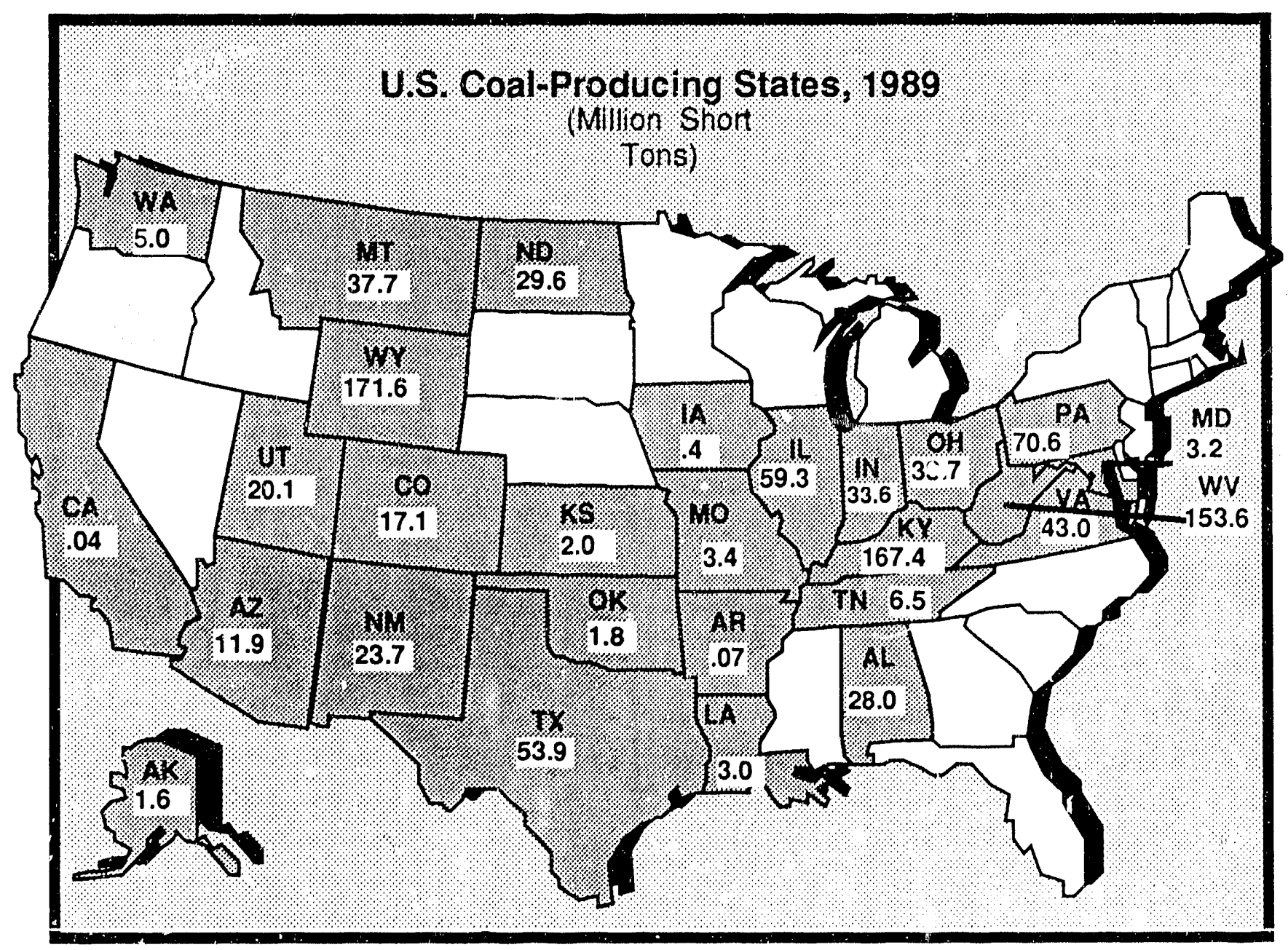




\section{Appendix B}

\section{State Coal Facts}

This appendix provides a ready reference of basic coalrelated information for each coal-producing State including detailed statistics for production, mining method, price, number of mines and miners, and consumption. Covering the period 1970 through 1989, the data show changes that have occurred since the oil embargo and the enactment of clean air standards in the early 1970 's. Data on coal-fired electric generating units and coal deposits are shown for 1989. A map appears for each State, with the coal-producing counties shaded and coal-fired electric generating plants indicated if coal is the primary fuel used at the plant.

Since 1970, coal has gained importance as a source of energy in the United States, its share of total energy consumption rising from almost 19 percent in 1970 to 23 percent in 1989 . Reflecting this growth, coal production rose from 613 million short tons in 1970 to 981 million short tons in 1989. Although States East of the Mississippi River have been the mainstay of U.S. coal production during the period, States West of the Mississippi River have gained importance as a source of coal. Coal production West of the Mississippi River rose from 45 million shert tons in 1970 to 382 million short tons in 1989. The magnitude of Western coal production is further emphasized in that Wyoming is now the leading producer for the second consecutive year. Of the seven States that reached record levels of production in 1989, five (Louisiana, New Mexico, Texas, Utah and Wyoming) are West of the Mississippi.

Coal consumption increased from 523 million short tons in 1970 to 889 million st.ort tons in 1989. Virtually all of the increase was due to the greater use of coal to generate electricity. This is illustrated by the fact that in 1989,86 percent of the total coal consumed was consumed by the electric utility industry, in contrast to 61 percent in 1970 . As a source of the total electricity generated, the share represented by coal increased from 46 percent in 1970 to 56 percent in 1989. Texas is still the top coal-consuming State $(91$ million short tons), primarily for electric power generation, followed by Ohio (61 million short tons) and Pennsylvania (59 million short tons). 
Table B1. Coal Production Trends by State (Thousand Short Tons)

\begin{tabular}{|c|c|c|c|c|c|c|c|c|}
\hline $\begin{array}{c}\text { Coal-Producing Region } \\
\text { and State }\end{array}$ & 1982 & 1983 & 1984 & 1985 & 1986 & 1987 & 1988 & 1989 \\
\hline Alabama & 26,556 & 23,812 & 27,088 & 27,797 & 25,826 & 25,540 & 26,518 & 27,992 \\
\hline Alaska & 833 & 786 & 859 & 1,433 & 1.570 & $1,49 c_{c}$ & 1,745 & 1,582 \\
\hline Arizona & 12,364 & 11,404 & 11,522 & 9,625 & 11,556 & 11,379 & 12,398 & 11,935 \\
\hline Arkansas & 161 & 88 & 82 & 80 & 167 & 84 & 276 & 70 \\
\hline 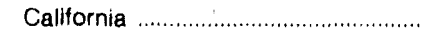 & . & - & - & 71 & - & 46 & 54 & 41 \\
\hline 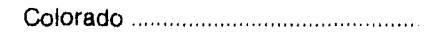 & 18,318 & 16,732 & 17,967 & 17,243 & 15,237 & 14,420 & 15,913 & 17,123 \\
\hline Georgia & 14 & 187 & 125 & - & - & - & - & - \\
\hline 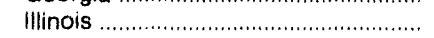 & 60,275 & 56,846 & 63,769 & 59,201 & 61,866 & 59,155 & 58,594 & 59,267 \\
\hline 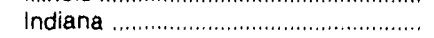 & 31,783 & 31,835 & 37,555 & 33,316 & 32,852 & 34,208 & 31,271 & 33,641 \\
\hline lowa & 566 & 385 & 527 & 591 & 484 & 468 & 341 & 430 \\
\hline 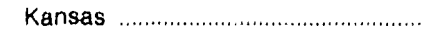 & 1,412 & 1,271 & 1,328 & 994 & 1,486 & 2,021 & 737 & $8 b 6$ \\
\hline 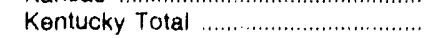 & 150.215 & 131,217 & 159,541 & 152,272 & 153,933 & 165,192 & 157,852 & 167,389 \\
\hline Eastern & 111,195 & $95,61 j$ & 117,274 & 113,251 & 112,732 & 119,906 & 117,524 & 125,739 \\
\hline Western & 39,020 & 35,602 & 42,267 & 39,021 & 41,201 & 45,285 & 40,328 & 41,649 \\
\hline 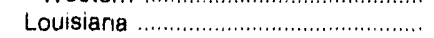 & - & - & - & 207 & 2,254 & 2,751 & 2,869 & 2,983 \\
\hline Maryland & 3,817 & 3,184 & 4,103 & 2,985 & 3,906 & 3,962 & 3,242 & 3,376 \\
\hline 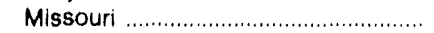 & 5,341 & 4,982 & 6,733 & 5,571 & 4,687 & 4,292 & 4,168 & 3,378 \\
\hline 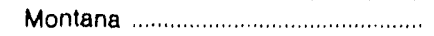 & 27,890 & 28,930 & 33,000 & 33,290 & 33,978 & 34,399 & 38,881 & 37,742 \\
\hline 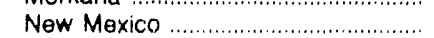 & 19,944 & 20,415 & 21,279 & 22,203 & 21,496 & 19,131 & 21,803 & 23,702 \\
\hline 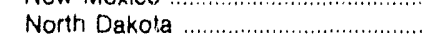 & 17,855 & 19,190 & 22,112 & 26,873 & 25,640 & 25,142 & 29,731 & 28,566 \\
\hline Ohio & 36,480 & 33,770 & 39,256 & 35,602 & 36,441 & 35,788 & 34,043 & 33,689 \\
\hline Oklahoma & 4,797 & 3,694 & 4,640 & 3,337 & $\begin{array}{r}30,44, \\
3,048\end{array}$ & 2,870 & 2,136 & $\begin{array}{r}00,000 \\
1,753\end{array}$ \\
\hline Pennsylvania Total ........................... & 79,359 & 69,828 & 77,494 & 71,408 & 71,648 & 70,423 & 70,645 & 70,596 \\
\hline Anthracite & 4,588 & 4,089 & 4,162 & 4,708 & 4,292 & 3,560 & 3,555 & 3,348 \\
\hline 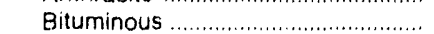 & 74,771 & 65,739 & 73,332 & 66,699 & 67,356 & 66,863 & 67,091 & 67,248 \\
\hline Tennessee & 7,450 & 6,640 & 7,313 & 7,446 & 6,870 & $6,44 ?$ & 6,510 & 6,480 \\
\hline Texas & 34,818 & 38,947 & 41,145 & 45,459 & 48,590 & 50,529 & 52,281 & 53,854 \\
\hline Utah & 17.029 & 11.768 & 12,323 & 12,780 & 14,269 & 16,508 & 18,163 & 20,102 \\
\hline Virginia & 39,778 & 35,027 & 40,368 & 40,940 & 41,178 & 44,543 & 45,886 & 43,006 \\
\hline 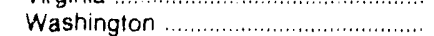 & 4,164 & 3,881 & 3,872 & 4,438 & 4,601 & 4,449 & 5,170 & 5,039 \\
\hline 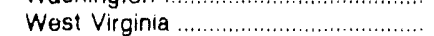 & 128,540 & 115,049 & 131,008 & 127,764 & 129.907 & 136,676 & 145,005 & 153.580 \\
\hline 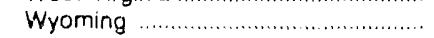 & 108,361 & 112,214 & 130,914 & 140,714 & 136,826 & 146,850 & 164,014 & 171,558 \\
\hline $\begin{array}{l}\text { Appalachian Total }{ }^{1} \ldots \ldots \ldots \ldots \ldots \ldots \ldots \ldots \\
\text { Interlor Total } \\
\text { Western Total' }\end{array}$ & $\begin{array}{l}433,199 \\
178,153 \\
228,758\end{array}$ & $\begin{array}{l}383,111 \\
173,651 \\
225,329\end{array}$ & $\begin{array}{l}444,029 \\
198,045 \\
253,846\end{array}$ & $\begin{array}{l}427,193 \\
187,778 \\
268,669\end{array}$ & $\begin{array}{l}428,508 \\
196,634 \\
265,172\end{array}$ & $\begin{array}{l}443,281 \\
201,663 \\
273,818\end{array}$ & $\begin{array}{l}449,373 \\
193,022 \\
307,871\end{array}$ & $\begin{array}{l}464,457 \\
197,880 \\
318,391\end{array}$ \\
\hline $\begin{array}{l}\text { East of the Mles. Rlver ................... } \\
\text { West of the Mlss. Rlver } \ldots \ldots \ldots \ldots \ldots \ldots \ldots\end{array}$ & $\begin{array}{l}564,257 \\
273,853\end{array}$ & $\begin{array}{l}507,395 \\
274,897\end{array}$ & $\begin{array}{l}587,620 \\
308,301\end{array}$ & $\begin{array}{l}558,730 \\
324,808\end{array}$ & $\begin{array}{l}564,427 \\
325,887\end{array}$ & $\begin{array}{l}581,929 \\
336,833\end{array}$ & $\begin{array}{l}579,565 \\
370,700\end{array}$ & $\begin{array}{l}599,015 \\
381,714\end{array}$ \\
\hline U.S. Total & 838,110 & 782,091 & 895,821 & 883,638 & 880,315 & 918,762 & 950,265 & 880,729 \\
\hline
\end{tabular}

1 For a defirition of coal-producing regions, see Appendix $C$.

Note: Excludes silt, culm, refuse bank, slurry dam, and dredge prodiction except for Pennsylvanic anthracite. Total may not equal sum of components becalse of independent rounding. Coal dat for 1989 have been revised and finalized.

Source: Energy Information Administration, Form EIA-6, "Coal Distribution Report"; and State mining agency coal production reports. 
Table B2. Rankings for Coal Consumption, Reserves, and Production by Coal-Producing State

\begin{tabular}{|c|c|c|c|c|c|c|c|c|c|}
\hline \multirow[b]{2}{*}{ State } & \multicolumn{5}{|c|}{1989 Consumption } & \multirow{2}{*}{$\begin{array}{c}\text { Demonatrated } \\
\text { Reserve Bsee } \\
\text { on January } 1 \\
1890\end{array}$} & \multicolumn{3}{|c|}{1989 Production } \\
\hline & $\begin{array}{l}\text { Electrlc } \\
\text { Utilities }\end{array}$ & $\begin{array}{l}\text { Coke } \\
\text { Planto }\end{array}$ & $\begin{array}{c}\text { Other } \\
\text { Industrial }\end{array}$ & $\begin{array}{c}\text { Resldential } \\
\text { and } \\
\text { Commerclal }\end{array}$ & Total & & Underground & Surlece & Total \\
\hline $\begin{array}{l}\text { Alabama } \\
\text { Alaska } \\
\text { Artzons } \\
\text { Arkaneas } \\
\text { Calffornia }\end{array}$ & $\begin{array}{l}10 \\
26 \\
13 \\
21 \\
-\end{array}$ & $\begin{array}{c}\because 4 \\
-1 / 1 \\
-1 \\
-\end{array}$ & $\begin{array}{r}12 \\
\quad 17 \\
22 \\
10\end{array}$ & $\begin{array}{l}16 \\
- \\
25 \\
23 \\
20\end{array}$ & $\begin{array}{r}9 \\
27 \\
14 \\
22 \\
26\end{array}$ & $\begin{array}{l}15 \\
13 \\
26 \\
25 \\
27\end{array}$ & $\begin{array}{l}7 \\
- \\
- \\
- \\
-\end{array}$ & $\begin{array}{l}13 \\
21 \\
12 \\
25 \\
26\end{array}$ & $\begin{array}{l}12 \\
23 \\
16 \\
26 \\
27\end{array}$ \\
\hline $\begin{array}{l}\text { Colorado } \\
\text { lilinols } \\
\text { Indiana } \\
\text { lowa } \\
\text { Kansas }\end{array}$ & $\begin{array}{r}14 \\
7 \\
4 \\
17 \\
16\end{array}$ & $\begin{array}{l}- \\
5 \\
1 \\
- \\
-\end{array}$ & $\begin{array}{r}21 \\
7 \\
2 \\
11 \\
25\end{array}$ & $\begin{array}{r}15 \\
4 \\
3 \\
10 \\
22\end{array}$ & $\begin{array}{r}15 \\
7 \\
4 \\
13 \\
19\end{array}$ & $\begin{array}{r}8 \\
2 \\
10 \\
18 \\
21\end{array}$ & $\begin{array}{r}9 \\
4 \\
11 \\
16 \\
-\end{array}$ & $\begin{array}{r}14 \\
11 \\
6 \\
24 \\
23\end{array}$ & $\begin{array}{r}15 \\
5 \\
10 \\
25 \\
24\end{array}$ \\
\hline $\begin{array}{l}\text { Kentucky } \\
\text { Loulslana } \\
\text { Maryland } \\
\text { Mlesourt } \\
\text { Montana }\end{array}$ & $\begin{array}{r}6 \\
20 \\
24 \\
8 \\
22\end{array}$ & $\begin{array}{l}8 \\
- \\
7 \\
- \\
-\end{array}$ & $\begin{array}{l}13 \\
16 \\
19 \\
15 \\
24\end{array}$ & $\begin{array}{r}7 \\
26 \\
17 \\
5 \\
18\end{array}$ & $\begin{array}{r}6 \\
21 \\
23 \\
10 \\
24\end{array}$ & $\begin{array}{r}5 \\
24 \\
23 \\
14 \\
1\end{array}$ & $\begin{array}{r}2 \\
- \\
12 \\
- \\
-\end{array}$ & $\begin{array}{r}2 \\
18 \\
22 \\
17 \\
5\end{array}$ & $\begin{array}{r}2 \\
21 \\
20 \\
10 \\
8\end{array}$ \\
\hline $\begin{array}{l}\text { Now Mexico } \\
\text { North Dakota } \\
\text { Onlo } \\
\text { Oklahoma } \\
\text { Pennsylvanla }\end{array}$ & $\begin{array}{r}15 \\
11 \\
2 \\
18 \\
3\end{array}$ & $\begin{array}{l}- \\
- \\
- \\
2\end{array}$ & $\begin{array}{r}26 \\
1 \\
3 \\
20 \\
5\end{array}$ & $\begin{array}{r}21 \\
11 \\
2 \\
24 \\
1\end{array}$ & $\begin{array}{r}16 \\
8 \\
2 \\
17 \\
3\end{array}$ & $\begin{array}{r}16 \\
11 \\
7 \\
19 \\
6\end{array}$ & $\begin{array}{r}14 \\
- \\
8 \\
15 \\
3\end{array}$ & $\begin{array}{r}9 \\
8 \\
10 \\
20 \\
7\end{array}$ & $\begin{array}{r}13 \\
11 \\
9 \\
22 \\
4\end{array}$ \\
\hline $\begin{array}{l}\text { Tennesee } \\
\text { Texas } \\
\text { Utah } \\
\text { Virginia } \\
\text { Wachington } \\
\text { West Virginia } \\
\text { Wyoming }\end{array}$ & $\begin{array}{r}12 \\
1 \\
19 \\
23 \\
25 \\
5 \\
9\end{array}$ & $\begin{array}{c}11 \\
- \\
9 \\
10 \\
- \\
6 \\
-\end{array}$ & $\begin{array}{r}6 \\
4 \\
18 \\
8 \\
23 \\
9 \\
14\end{array}$ & $\begin{array}{r}8 \\
19 \\
6 \\
9 \\
14 \\
12 \\
13\end{array}$ & $\begin{array}{r}12 \\
1 \\
18 \\
20 \\
25 \\
5 \\
11\end{array}$ & $\begin{array}{r}22 \\
9 \\
12 \\
17 \\
20 \\
4 \\
3\end{array}$ & $\begin{array}{r}10 \\
- \\
6 \\
5 \\
- \\
1 \\
13\end{array}$ & $\begin{array}{r}19 \\
3 \\
- \\
15 \\
16 \\
4 \\
1\end{array}$ & $\begin{array}{r}17 \\
6 \\
14 \\
7 \\
18 \\
3 \\
1\end{array}$ \\
\hline
\end{tabular}

Sources: Energy Information Administration, Coal Production; Quartorly Coal Report; Weekly Coal Production; and Coal Data: A Reterence. 
Table B3. U.S. Coal Production Ranked by State, 1987-1989

(Thousand Short Tons)

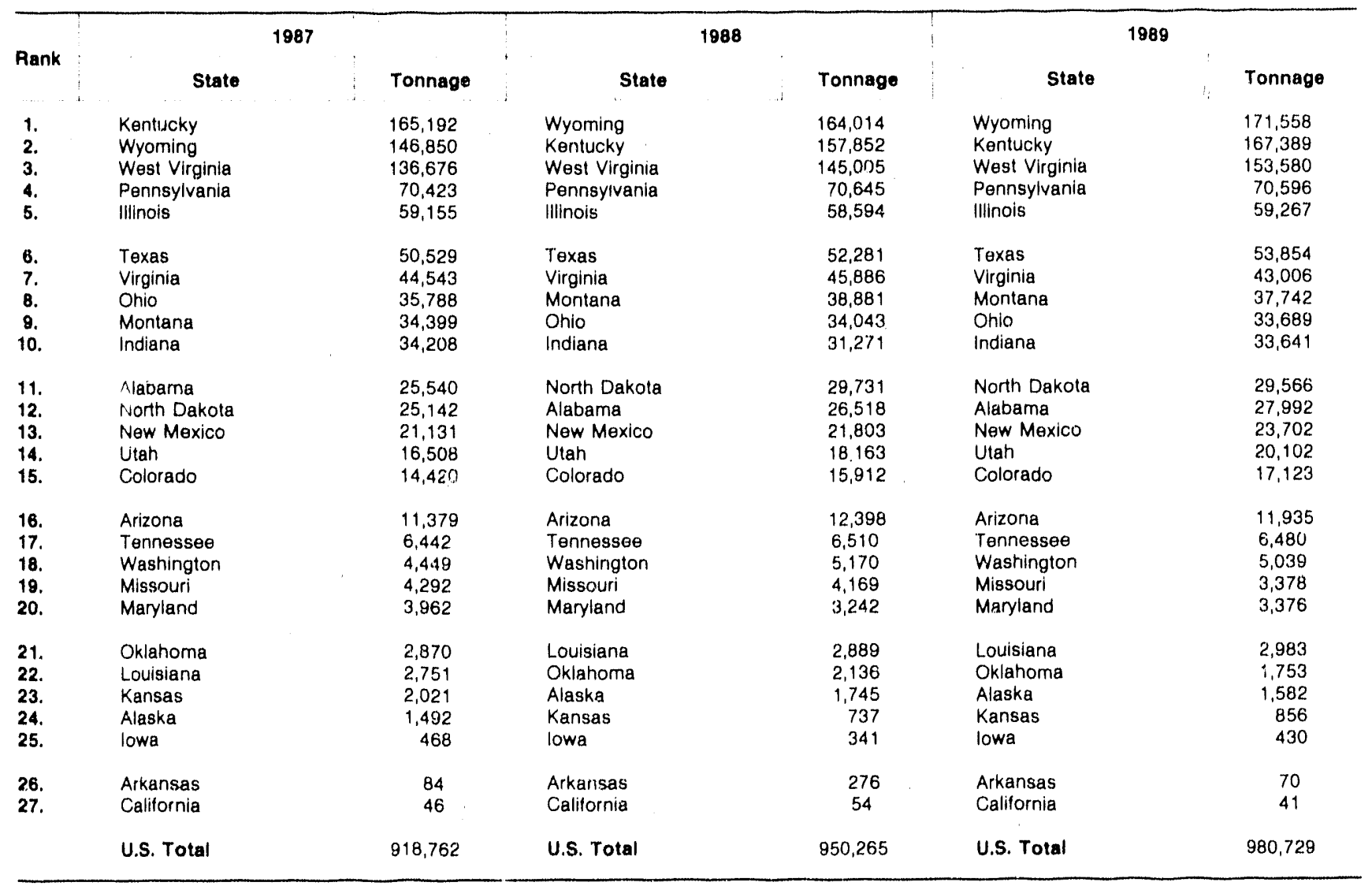

Source: Energy Information Administration, COal Production, DOE/EIA-0118, various issues. 
Table B4. U.S. Coal Consumption Ranked by State, 1987-1989

(Thousand Short Tons)

\begin{tabular}{|c|c|c|c|c|c|c|}
\hline \multirow{2}{*}{ Rank } & \multicolumn{2}{|c|}{1987} & \multicolumn{2}{|c|}{1988} & \multicolumn{2}{|c|}{1989} \\
\hline & State & Tonnage & State & Tonnage & State & Tonnage \\
\hline $\begin{array}{l}1 . \\
2 . \\
3 . \\
4 . \\
5 .\end{array}$ & $\begin{array}{l}\text { Texas } \\
\text { Ohio } \\
\text { Pennsylvanla } \\
\text { Indiana } \\
\text { Michigan }\end{array}$ & $\begin{array}{l}82,915 \\
59,350 \\
55,305 \\
52,335 \\
35,865\end{array}$ & $\begin{array}{l}\text { Texas } \\
\text { Ohio } \\
\text { Pennsylvania } \\
\text { Indiana } \\
\text { West Virginia }\end{array}$ & $\begin{array}{l}86,689 \\
61,096 \\
58,639 \\
56,762 \\
36,435\end{array}$ & $\begin{array}{l}\text { Texas } \\
\text { Ohilo } \\
\text { Pennsylvania } \\
\text { Indlana } \\
\text { West Virginia }\end{array}$ & $\begin{array}{l}90,989 \\
61,016 \\
58,526 \\
58,203 \\
37,186\end{array}$ \\
\hline $\begin{array}{c}6 . \\
7 . \\
8 . \\
9 . \\
10 .\end{array}$ & $\begin{array}{l}\text { West Virginia } \\
\text { lllinols } \\
\text { Kentucky } \\
\text { Georgia } \\
\text { Alabama }\end{array}$ & $\begin{array}{l}34,815 \\
34,632 \\
32,023 \\
29,126 \\
26,632\end{array}$ & $\begin{array}{l}\text { Michigan } \\
\text { Kentucky } \\
\text { Illinois } \\
\text { Georgia } \\
\text { North Dakota }\end{array}$ & $\begin{array}{l}35,355 \\
35,176 \\
32,979 \\
28,654 \\
28,028\end{array}$ & $\begin{array}{l}\text { Michigan } \\
\text { Kentucky } \\
\text { Illinois } \\
\text { Georgia } \\
\text { North Dakota }\end{array}$ & $\begin{array}{l}34,932 \\
32,792 \\
32,374 \\
27,918 \\
27,401\end{array}$ \\
\hline $\begin{array}{l}11 . \\
12 . \\
13 . \\
14 . \\
15 .\end{array}$ & $\begin{array}{l}\text { Tennessee } \\
\text { Missouri } \\
\text { Wyoming } \\
\text { North Dakota } \\
\text { Florida }\end{array}$ & $\begin{array}{l}24,782 \\
24,764 \\
21,399 \\
24,101 \\
23,644\end{array}$ & $\begin{array}{l}\text { Alabama } \\
\text { Missouri } \\
\text { Wyoming } \\
\text { Tennesse日 } \\
\text { Florida }\end{array}$ & $\begin{array}{l}26,365 \\
26,119 \\
25,424 \\
25,152 \\
24,595\end{array}$ & $\begin{array}{l}\text { Alabama } \\
\text { Missouri } \\
\text { Florida } \\
\text { Wyoming } \\
\text { Tennessee }\end{array}$ & $\begin{array}{l}27,261 \\
26,297 \\
25,447 \\
23,952 \\
23,496\end{array}$ \\
\hline $\begin{array}{l}16 . \\
17 . \\
18 . \\
19 . \\
20 .\end{array}$ & $\begin{array}{l}\text { North Carolina } \\
\text { Wisconsin } \\
\text { Kansas } \\
\text { lowa } \\
\text { Colorado }\end{array}$ & $\begin{array}{l}19,965 \\
19,652 \\
15,194 \\
15,191 \\
15,007\end{array}$ & $\begin{array}{l}\text { North Carolina } \\
\text { Wisconsin } \\
\text { Minnesota } \\
\text { lowa } \\
\text { Colorado }\end{array}$ & $\begin{array}{l}20,506 \\
20,038 \\
17,231 \\
16,114 \\
15,860\end{array}$ & $\begin{array}{l}\text { North Carolina } \\
\text { Wisconsin } \\
\text { Minnesota } \\
\text { lowa } \\
\text { Arizona }\end{array}$ & $\begin{array}{l}22,239 \\
19,922 \\
17,584 \\
17,103 \\
16,871\end{array}$ \\
\hline $\begin{array}{l}21 . \\
22 . \\
23 . \\
24 . \\
25 .\end{array}$ & $\begin{array}{l}\text { Minnesota } \\
\text { New Mexico } \\
\text { Oklahoma } \\
\text { Arizona } \\
\text { Virginia }\end{array}$ & $\begin{array}{l}14,504 \\
14,395 \\
13,476 \\
13,375 \\
13,227\end{array}$ & $\begin{array}{l}\text { Oklahoma } \\
\text { Kansas } \\
\text { Now Moxico } \\
\text { Arizona } \\
\text { Utah }\end{array}$ & $\begin{array}{l}15,006 \\
14,951 \\
14,715 \\
14,525 \\
14,513\end{array}$ & $\begin{array}{l}\text { Colorado } \\
\text { New Mexico } \\
\text { Oklahorna } \\
\text { Utah } \\
\text { Karisas }\end{array}$ & $\begin{array}{l}16,393 \\
15,295 \\
15,086 \\
15,044 \\
14,963\end{array}$ \\
\hline $\begin{array}{l}26 . \\
27 . \\
28 . \\
29 . \\
30 .\end{array}$ & $\begin{array}{l}\text { Arkansas } \\
\text { Utah } \\
\text { South Carolina } \\
\text { New York } \\
\text { Maryland }\end{array}$ & $\begin{array}{l}12,066 \\
11,807 \\
11,701 \\
11,471 \\
11,311\end{array}$ & $\begin{array}{l}\text { Virginia } \\
\text { New York } \\
\text { Louisiana } \\
\text { Arkansas } \\
\text { South Carolina }\end{array}$ & $\begin{array}{l}13,430 \\
12,955 \\
12,848 \\
12,555 \\
11,937\end{array}$ & $\begin{array}{l}\text { Virginia } \\
\text { New York } \\
\text { Louisiana } \\
\text { South Carolina } \\
\text { Arkansas }\end{array}$ & $\begin{array}{l}14,279 \\
14,105 \\
12,471 \\
11,981 \\
11,547\end{array}$ \\
\hline $\begin{array}{l}31 . \\
32 . \\
33 . \\
34 . \\
35 .\end{array}$ & $\begin{array}{l}\text { Louisiana } \\
\text { Montana } \\
\text { Nevada } \\
\text { Nebraska } \\
\text { Washington }\end{array}$ & $\begin{array}{l}10,391 \\
7,730 \\
6,920 \\
6,744 \\
5,819\end{array}$ & $\begin{array}{l}\text { Maryland } \\
\text { Montana } \\
\text { Nevada } \\
\text { Nebraska } \\
\text { Washington }\end{array}$ & $\begin{array}{l}11,757 \\
10,634 \\
8,276 \\
8,057 \\
5,929\end{array}$ & $\begin{array}{l}\text { Maryland } \\
\text { Montana } \\
\text { Nevada } \\
\text { Nebraska } \\
\text { Washington }\end{array}$ & $\begin{array}{l}11,541 \\
10,458 \\
7,667 \\
7,587 \\
5,843\end{array}$ \\
\hline $\begin{array}{l}38 . \\
37 . \\
38 . \\
39 . \\
40 .\end{array}$ & $\begin{array}{l}\text { Mississippi } \\
\text { Massachusetts } \\
\text { Now Jersey } \\
\text { Delaware } \\
\text { California }\end{array}$ & $\begin{array}{l}4,846 \\
4,487 \\
3,434 \\
2,710 \\
1,934\end{array}$ & $\begin{array}{l}\text { Mississippi } \\
\text { Massachusetts } \\
\text { New Jersey } \\
\text { Delaware } \\
\text { South Dakota }\end{array}$ & $\begin{array}{l}5,136 \\
4,463 \\
3,295 \\
2,686 \\
2,591\end{array}$ & $\begin{array}{l}\text { Mussachusetts } \\
\text { Mississippl } \\
\text { New Jersey } \\
\text { California } \\
\text { South Dakota }\end{array}$ & $\begin{array}{l}4,641 \\
3,831 \\
3,545 \\
2,551 \\
2,541\end{array}$ \\
\hline $\begin{array}{l}41 . \\
42 . \\
43 . \\
44 . \\
45 .\end{array}$ & $\begin{array}{l}\text { New Hampshire } \\
\text { South Dakota } \\
\text { Connecticut } \\
\text { Idaho } \\
\text { Alaska }\end{array}$ & $\begin{array}{r}1,176 \\
1,101 \\
815 \\
494 \\
274\end{array}$ & $\begin{array}{l}\text { California } \\
\text { New Hampshire } \\
\text { Connecticut } \\
\text { Alaska } \\
\text { Idaho }\end{array}$ & $\begin{array}{r}2,209 \\
1,229 \\
881 \\
727 \\
527\end{array}$ & $\begin{array}{l}\text { Delaware } \\
\text { New Hampshire } \\
\text { Connecticut } \\
\text { Idaho } \\
\text { Oregon }\end{array}$ & $\begin{array}{r}2,357 \\
1,183 \\
866 \\
533 \\
396\end{array}$ \\
\hline $\begin{array}{l}46 . \\
47 . \\
48 . \\
49 . \\
50 . \\
51 .\end{array}$ & $\begin{array}{l}\text { Maine } \\
\text { Oregon } \\
\text { Dist Columbia } \\
\text { Hawaii } \\
\text { Vermont } \\
\text { Rhode Island }\end{array}$ & $\begin{array}{l}273 \\
205 \\
70 \\
63 \\
12 \\
5\end{array}$ & $\begin{array}{l}\text { Maine } \\
\text { Oregon } \\
\text { Rhode Island } \\
\text { Hawaii } \\
\text { Dist Columbia } \\
\text { Vermont }\end{array}$ & $\begin{array}{l}277 \\
176 \\
175 \\
50 \\
31 \\
11\end{array}$ & $\begin{array}{l}\text { Alaska } \\
\text { Maine } \\
\text { Dist Columbia } \\
\text { Hawail } \\
\text { Rhode Island } \\
\text { Vermont }\end{array}$ & $\begin{array}{l}299 \\
271 \\
60 \\
32 \\
27 \\
9\end{array}$ \\
\hline & Unknown & 411 & Unknown & 124 & Unknown & 581 \\
\hline & U.S. Total & 836,941 & U.S. Total & 883,664 & U.S. Total & 889,481 \\
\hline
\end{tabular}

Sources: Energy Information Administration, Quarterly COal Report, DOE/EIA-0121, various issues. 
Table B5. U.S. Demonstrated Reserve Base on January 1, 1990, by Mining Method and Ranked by State Totai

(Million Short Tons)

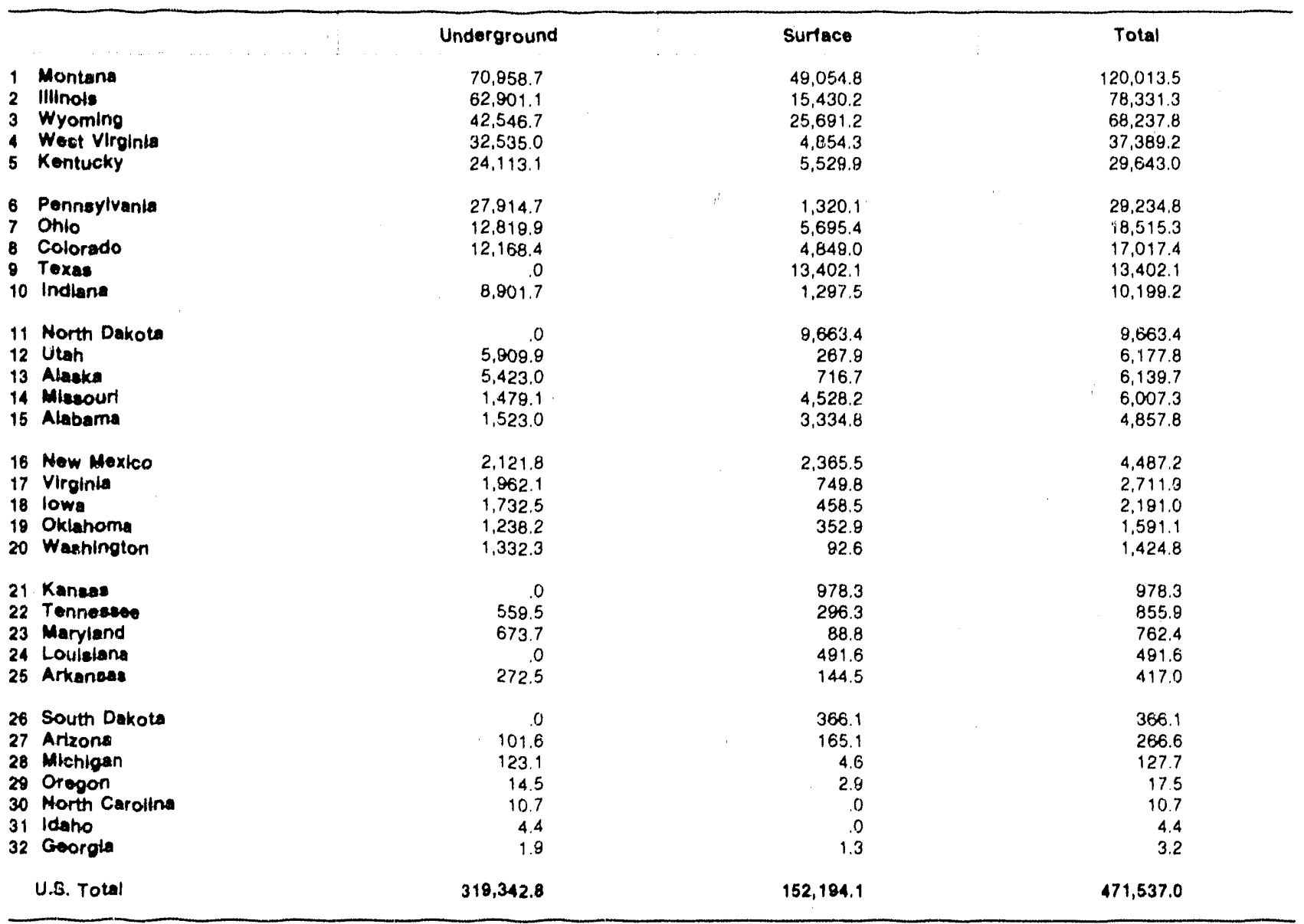

Sources: State geological and mineral resource surveys, and other geological reports. 


\section{Coal Highlights}

Production

(thousand short tons)

Value

(million dollars)

Number of Employees

Consumption

(thousand short tons)

Coal-Fired Electric Generating Units

Number

Summer Capability (megawatts)

$\%$ of Total Summer Capability

Generation (gigawatthours)

Coal Deposits

Area (square miles)

Percent of State Land
27,992

1,153

6,505

27,261

11,171

57.5

52,601

9,700

19

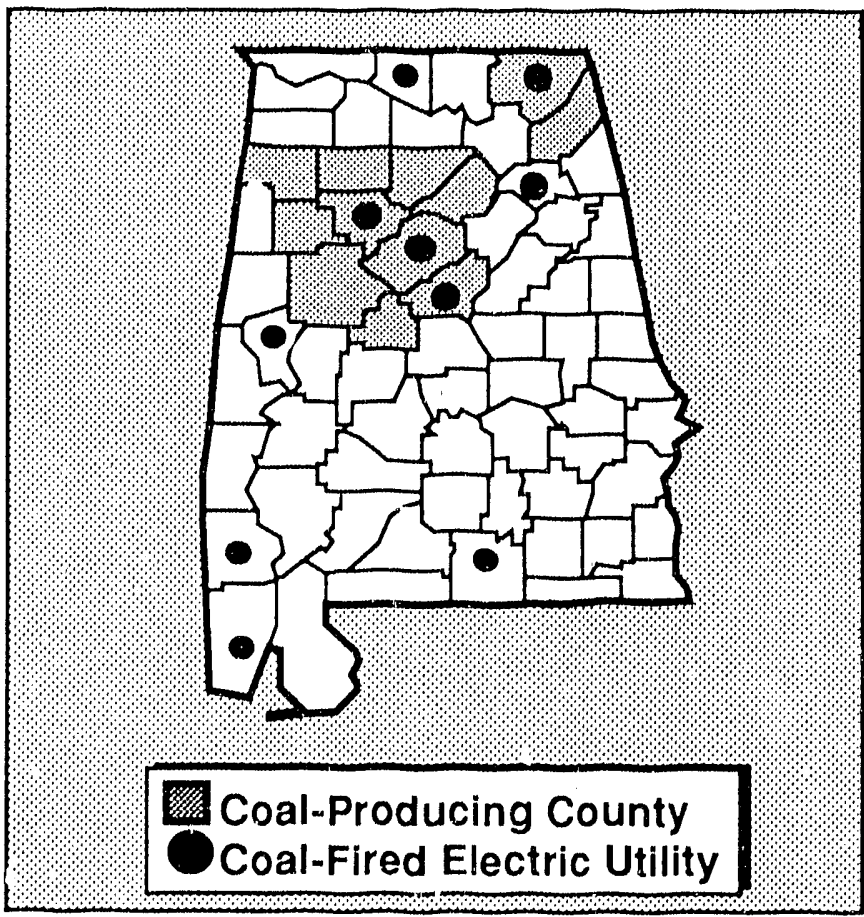

Table B6. Alabama Coal Facts

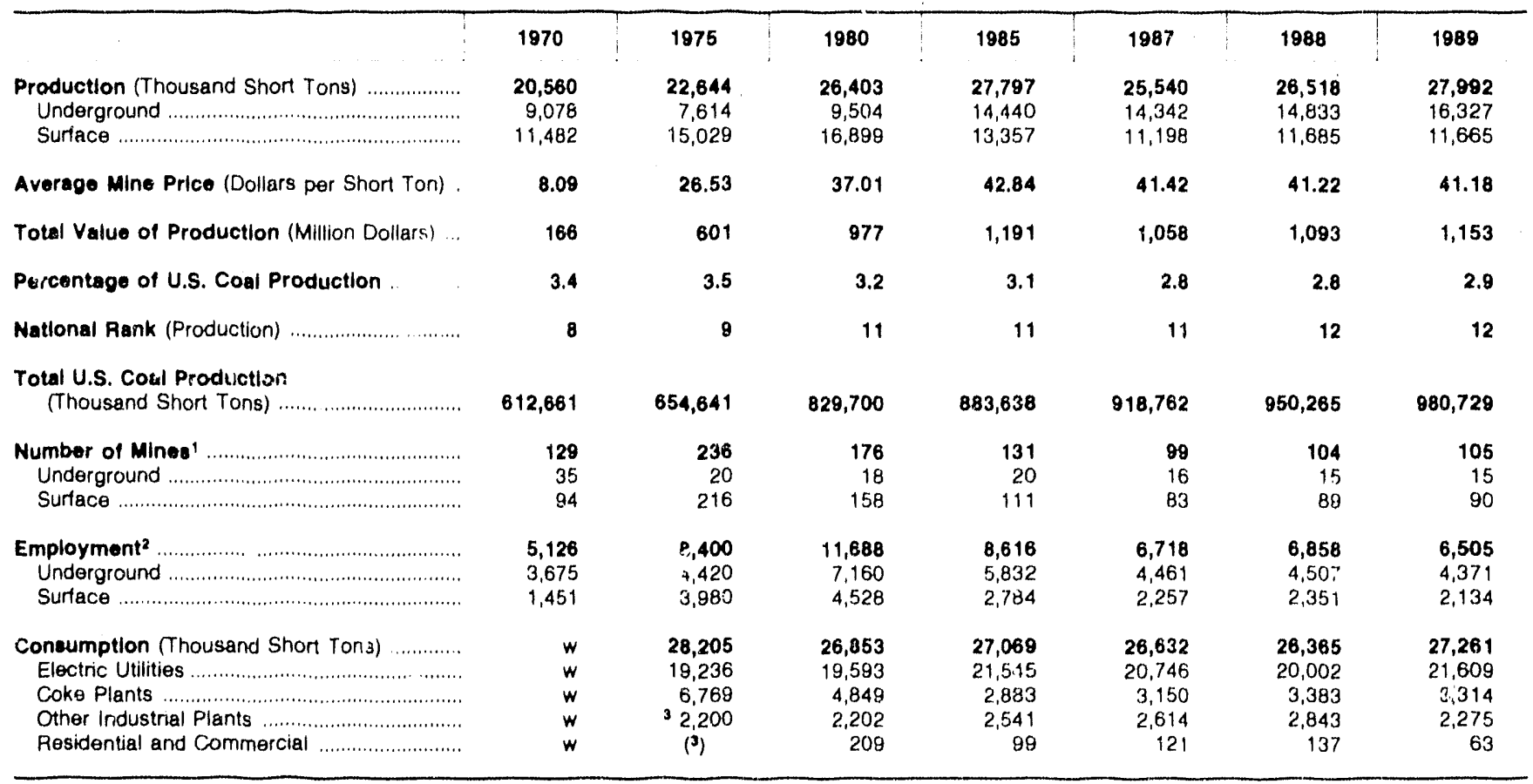

1 For 1980, includes only mines that produced 10,000 or more tons.

2 Beginning in 1980 , includes only mines that produced 10,000 or more tons

3 Consumption data at Sther Industrial Plants and in Residential and Commercial Sector are combined to avoid disclosure of individual company data.

- Withheid to avoid disclosure of individual company data.

Note: Total may not equal sum of components because of indapendent rounding

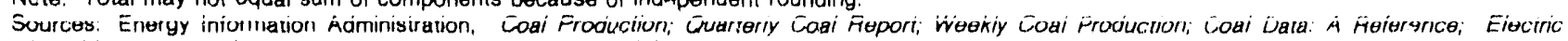
Power Monthly 1989; and Inventory of Power Plants in the United States 1989; Bureau of Mines, U.S. Department of Interior, Minerals Yeartook; Intormation Circular 8705: Historical Fuels and Energy Consumption Data, 1960-72, United States by States and Census Districts West of the Mississippi; and Ge0. logical Survey Bulletin 1412: Coal Resources of the United States, January 1, 1974. 


\section{Alaska}

\section{Coal Highlights}

Production

(thousand short tons)

Value

(million dollars)

Number of Employees

Consumption

(thousand short tons)

Coal-Fired Electric Generating Units

Number

Summer Capability (megawatts)

$\%$ of Total Summer Capability

Generation (gigawatthours)

\section{Coal Deposits}

Area (square miles)

Percent of State Land
W

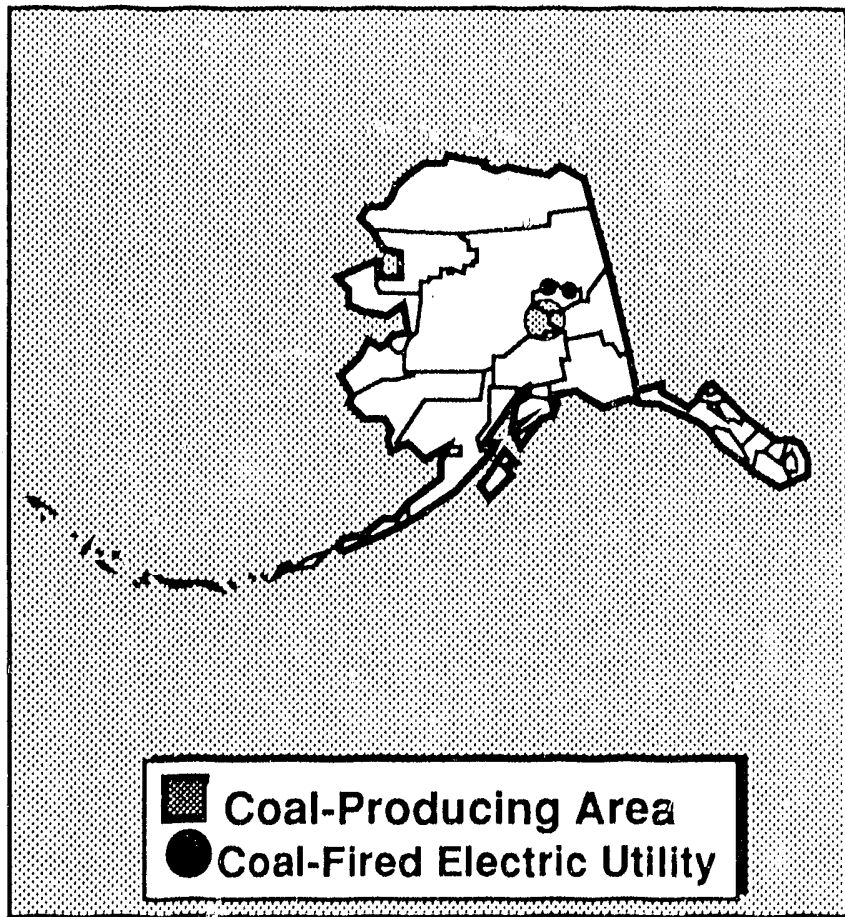

Table B7. Alama Coal Facts

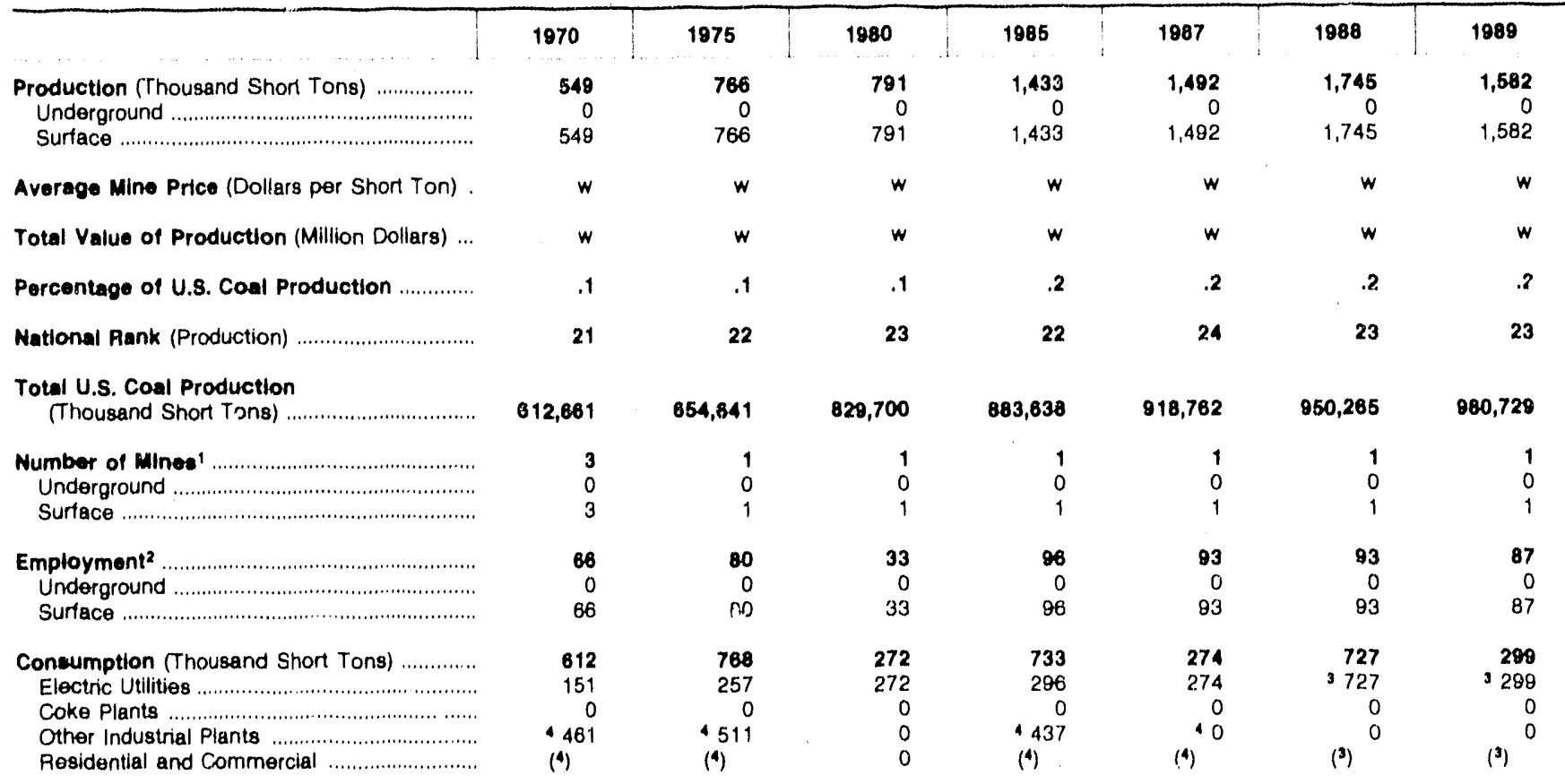

1 For 1980 , includes only mines that produced 10,000 or more tons

2 Beginning in 1880 , includes only mines that produced $; 0,000$ or more tone

- Consumption data at Electric Utilities and in Fesidential and Commercial Sector are combiried to avoid disclosure of individual company data.

4 Consumption data at Other Industrial Plants and in Residential and Commercial Sector are combined io avoid disclosure of individual company data.

- Withhetd to avoid disclosure of individual company data.

Note: Total may not equal sum of components because of independent rounding

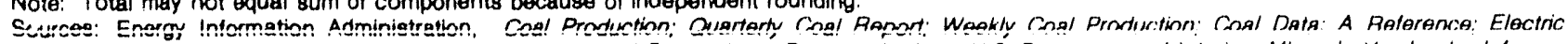
Power Monthly 1989; and invintory of Power Plants in the United States 1989; Bureau of Mines, U.S. Department of Interior, Minerals Yearbook; Informason Circular 8705: Historical Fuels and Energy Consumption Data, 1960-72, United States by states ana Census Districts West of the Mississippi; and Geo. togical Survey Bulletin 1412: Coal Resources of the United States, January 1, 1974. 


\section{Arizona}

\section{Coal Highlights}

Production

(thousand short tons)

11,935

Value

(million dollars)

Number of Employees

780

Consumption

(thousand short tons)

16,871

Coal-Fired Electric Generating Units

Number

Summer Capability (megawatts)

$\%$ of Total Summer Capability

4,756

Generation (gigawatthours)

32,364

\section{Coal Deposits}

Area (square miles)

Percent of State Land

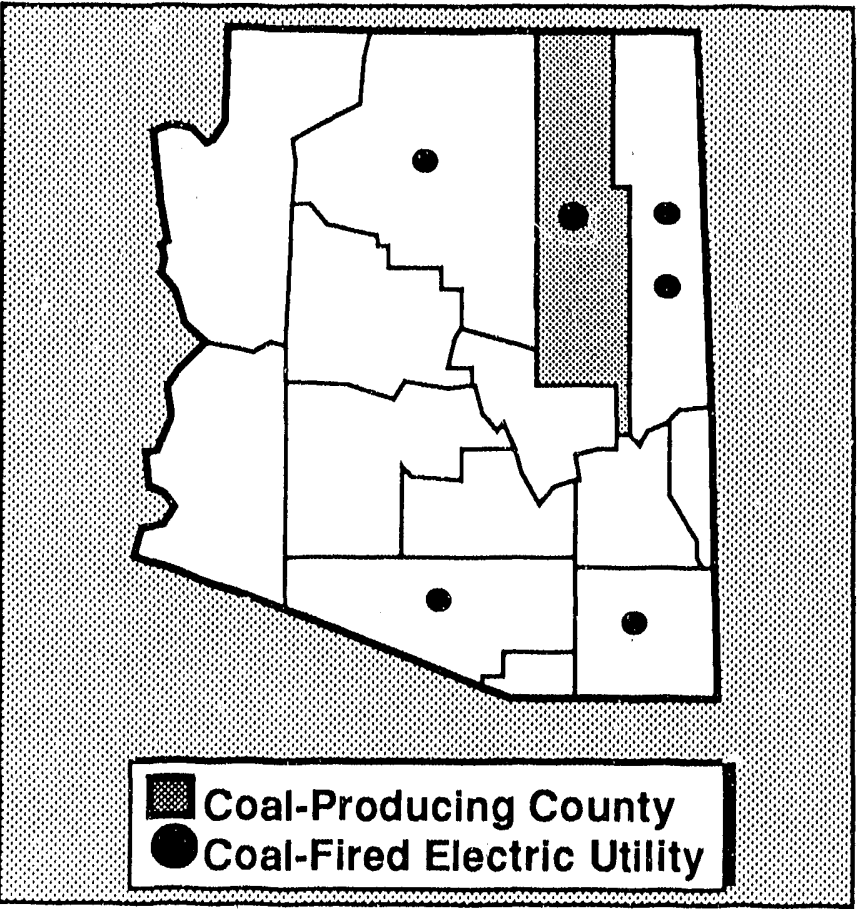

Table B8. Arizona Coal Facts

\begin{tabular}{|c|c|c|c|c|c|c|c|}
\hline$\ldots 1$ & 1970 & 1975 & 1980 & 1985 & 1987 & 19887 & 1889 \\
\hline Production (Thousand Short Tons) ................. & 132 & 6,986 & 10,905 & 9,625 & 11,379 & 12,398 & 11,835 \\
\hline 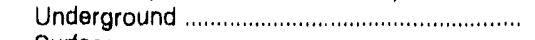 & 0 & & 0 & 0 & 0 & 0 & 0 \\
\hline Surface & 132 & 6,886 & 10,905 & 9,625 & 11,379 & 12,398 & 11,935 \\
\hline Average Mine Price (Dollars per Short Ton) & $w$ & $w$ & $w$ & $w$ & $w$ & $w$ & $w$ \\
\hline Total Value of Production (Million Dollars) ... & $w$ & $w$ & $w$ & $w$ & $w$ & $w$ & $w$ \\
\hline Percentage of U.S. Coal Production .............. & (') & 1.1 & 1.3 & 1.1 & 1.2 & 1.3 & 1.2 \\
\hline 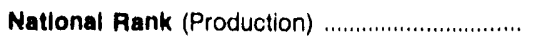 & 23 & 16 & 18 & 16 & 16 & 16 & 16 \\
\hline \multicolumn{8}{|l|}{ Total U.S. Coal Production } \\
\hline 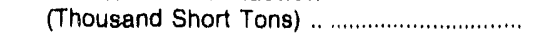 & 612,661 & 654,641 & 829,700 & 683,638 & 918,762 & 950,265 & 980,729 \\
\hline 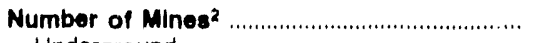 & 1 & 2 & 2 & 2 & 2 & 2 & 2 \\
\hline 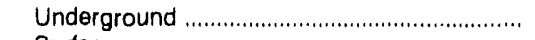 & 0 & 0 & 0 & 0 & 0 & 0 & 0 \\
\hline Surtace & 1 & 2 & 2 & 2 & 2 & 2 & 2 \\
\hline 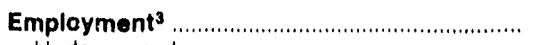 & 75 & 450 & 897 & 885 & 900 & 884 & 780 \\
\hline 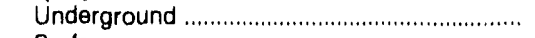 & 0 & 0 & 0 & 0 & 0 & 0 & 0 \\
\hline 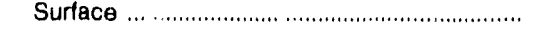 & 75 & 450 & 897 & 885 & 900 & 984 & 780 \\
\hline Consumption (Thousand Short Tons) ............. & $w$ & 3,985 & 11,558 & 16,384 & 13,375 & 14,525 & 16,871 \\
\hline 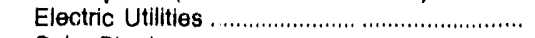 & $w$ & 3,873 & 10,915 & 14,448 & 12,706 & 13,932 & 16,182 \\
\hline 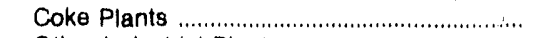 & $w$ & 0 & 0 & 0 & 0 & 0 & 0 \\
\hline 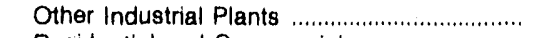 & $w$ & 4112 & 4643 & 4,916 & 4669 & 4593 & 689 \\
\hline Residential and Commercial ............................. & $w$ & $(4)$ & $(4)$ & (4) & $(4)$ & $(4)$ & (4) \\
\hline
\end{tabular}

1 Less than 0.05 percent.

2 For 1980 , includes only mines that produced 10,000 or more tons.

3 Beginning in 1980 , includes only mines that produced 10,000 or more tons.

- Consumption data at Other Industrial Plants and in Residential and Commersial Sector are combined to avciJ disclosure of individual company data.

withheld to avoid disclosure of individual company data.

Note: Total may not equal sum of comporients because of independent rounding.

Sources: Energy Inforrnation Administration, Coal Production; Quarteriv Coal Report: Weekly Coal Proxtuction: Casal Mata A Raferenca: Electric Power Monthly 1989; and Inventory of Power Hiants in the United States 1989; Bureau of Mines, U.S. Department of Interior, Minerals Yeartook; Intorma. tion Circular 8705: Historical Fuels and Energy Consumption Data, 1960-72. United States by States and Census Districts West of the Mississippi; and Geo. logical Survey Bulletin 1.112: Coal Resources of the United States, January 1, 1974. 


\section{Arkansas}

\section{Coal Highlights}

Production

(thousand short tons)

Value

(million dollars)

Number of Employees

Consumption

(thousand short tons)

Coal-Fired Electric Generating Units

Number

Summer Capability (megawatts)

$\%$ of Total Summer Capability

Generation (gigawatthours)

Coal Deposits

Area (square miles)

Percent of State Land

70

w

8

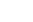

7

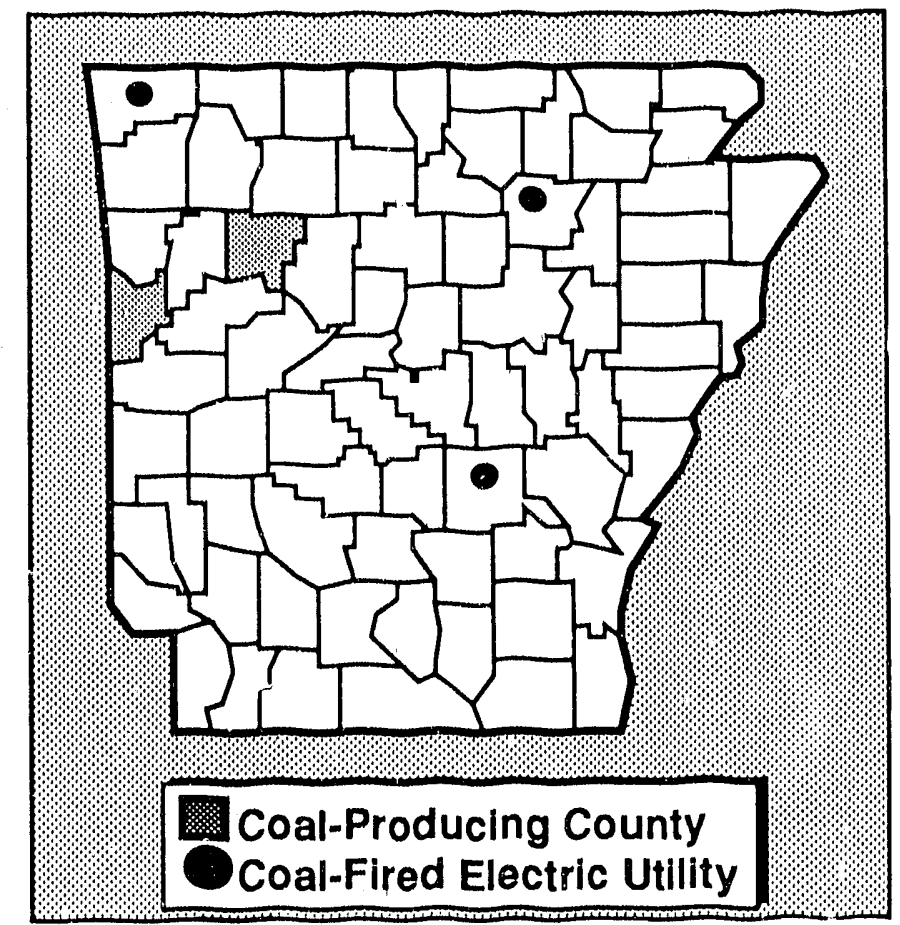

Table B9. Arkansás Coal Facts

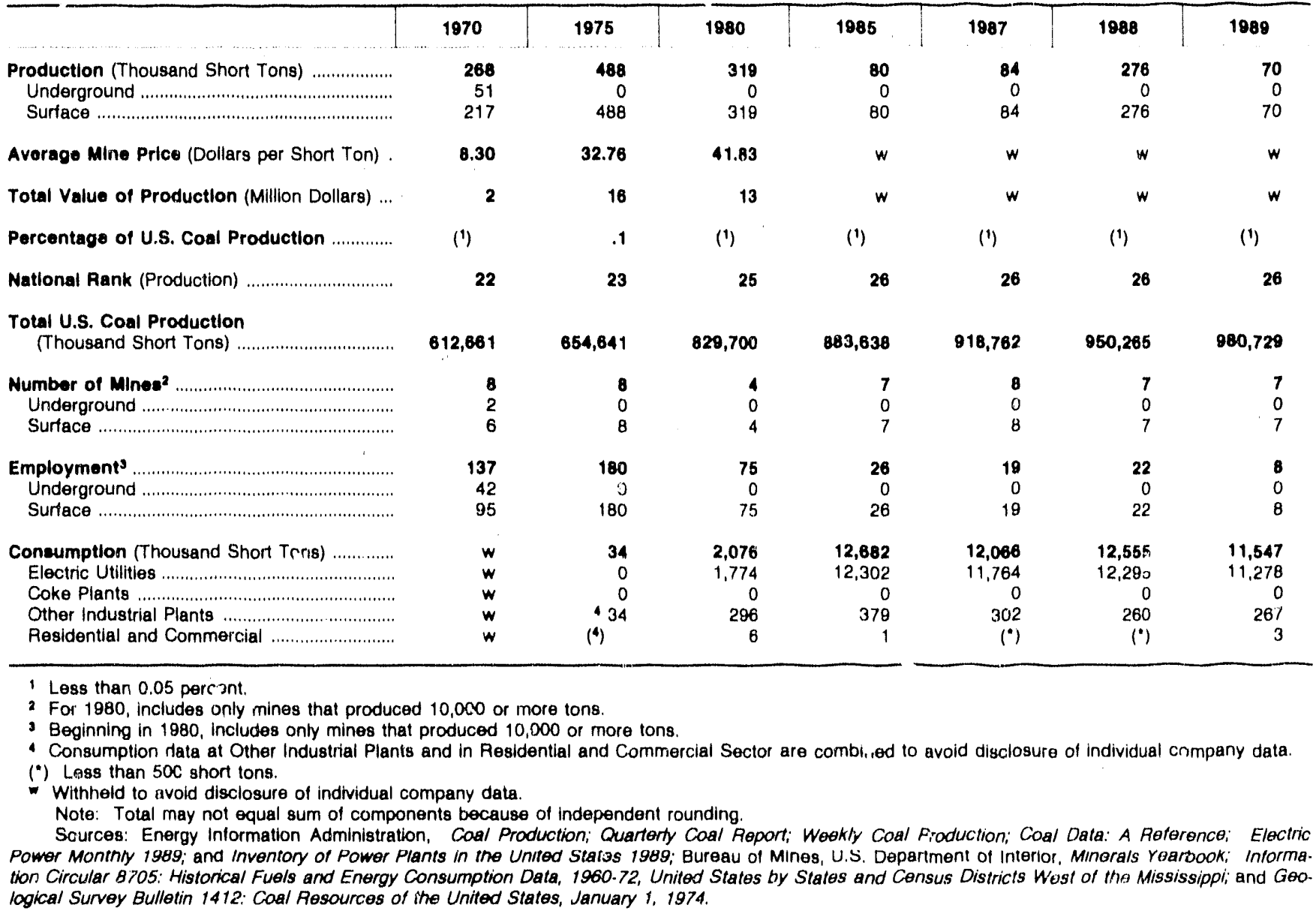




\section{California}

\section{Coal Highlights}

Production

(thousand short tons)

Value

(million dollars)

Number of Employees

Consumption

(thousand short tons)

Coal-Fired Electric Generating Units

Number

Summer Capability (megawatts)

$\%$ of Total Summer Capability

Generation (gigawatthours)

Coal Deposits

Area (square miles)

Percent of State Land
41

w

2,551

0

0

0

0.1

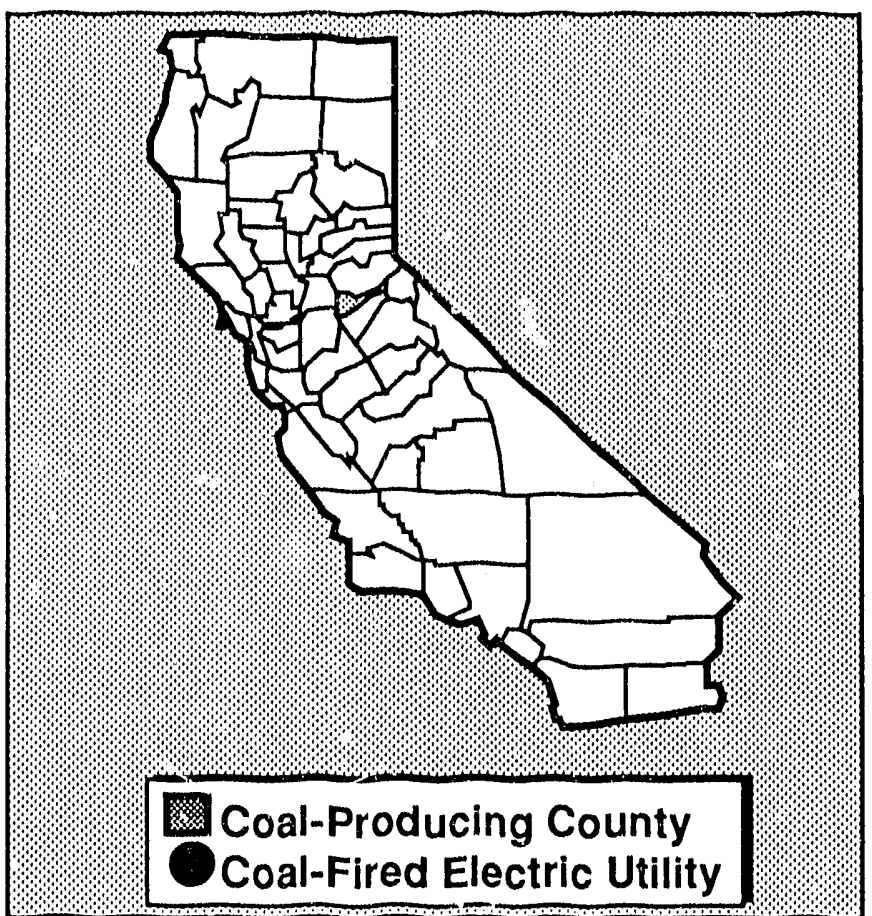

Table B10. California Coal Facts

\begin{tabular}{|c|c|c|c|c|c|c|c|}
\hline & 1970 & 1975 & 1980 & 1985 & 1987 & 1988 & 1989 \\
\hline Production (Thousand Short Tons) .................... & 0 & 0 & 0 & 71 & $\Delta 6$ & 54 & 41 \\
\hline 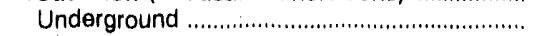 & 0 & 0 & 0 & 0 & 0 & 0 & 0 \\
\hline 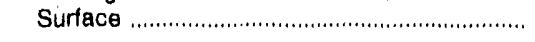 & 0 & 0 & 0 & 71 & 46 & 54 & 41 \\
\hline Average Mine Price (Dollars per Short Ton) & .00 & .00 & .00 & 6.05 & $w$ & w & $w$ \\
\hline Total Value of Production (Million Dollars) ... & 0 & 0 & 0 & 0 & w & $w$ & $w$ \\
\hline Percentage of U.S. Coal Production ................ & .0 & .0 & .0 & (1) & .0 & (1) & (1) \\
\hline 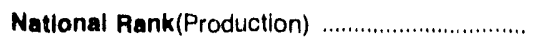 & 0 & 0 & 0 & 27 & 27 & 27 & 27 \\
\hline $\begin{array}{l}\text { Total U.S. Coal Production } \\
\text { (Thousand Short Tons) }\end{array}$ & 612,661 & 654,641 & 829,700 & 883,638 & 918,762 & 950,265 & $y 80,729$ \\
\hline Number of Mines $2 \ldots$ & 0 & 0 & 0 & 1 & 1 & 1 & 1 \\
\hline Underground .............................................. & 0 & 0 & 0 & 0 & 0 & 0 & 0 \\
\hline 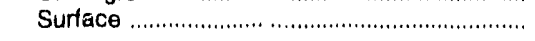 & 0 & 0 & 0 & 1 & 1 & 1 & 1 \\
\hline Employment ${ }^{3}$ & 0 & 0 & 0 & 9 & 37 & 8 & 5 \\
\hline Underground ................. & 0 & 0 & 0 & 0 & 0 & 0 & 0 \\
\hline 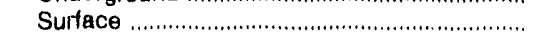 & 0 & 0 & 0 & 9 & 37 & 8 & 5 \\
\hline Consumption (Thousand Short Tons) .................. & 2,317 & 2,151 & 2,669 & 1,942 & 1,934 & 2,209 & 2,551 \\
\hline 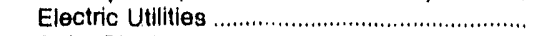 & 0 & 0 & 0 & 0 & 0 & 0 & 0 \\
\hline 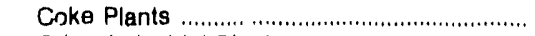 & 0 & 0 & 1,510 & 0 & 0 & 0 & 0 \\
\hline Other Industrial Plants .............................. & 2,189 & 2,151 & 1,155 & 1,889 & 1,834 & 42,203 & 42,551 \\
\hline Residential and Commercial ......................... & 128 & 0 & 4 & 5,3 & 0 & $(4)$ & $(4)$ \\
\hline
\end{tabular}

1 Less than 0.05 percent.

2 For 1980 , includes only mines that produced 10,000 or more tons.

3 Beginning in 1980, lincludes only mines that produced 10,000 or more tons.

- Consumption data at Other Industrial Plants and in Residential and Commercial Sector are combined to avoid nisclosure of individual company data.

w Withheld to avoid discicsure of individual company data.

Note: Total may not equal sum cf components because of independent rounding.

Sources: Energy Information Administration, Caal Production; Quarterly Cual Report; Weekly Coal Pro uction;Coal Data: A Heterence; Electric Power

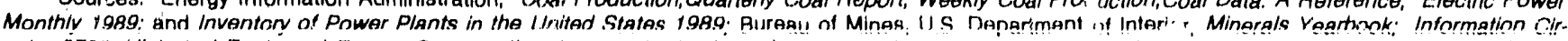
cular 8705: Historical Fuels aind Energy Consumption Data, 1960.72, United States by States and Census Districts Vest of the Mississippi: andGeological Surver Bulletin 1412: Caal Resources of the United States, January 1, 1974. 


\section{Colorado}

\section{Coal Highlights}

Production

(tho'sand short tons)

17,123

Value

(million dollars)

405

Number of Employees

2,019

Consumption

(thousand short tons)

16,393

Coal-Fired Electric Generating Units

Number

Summer Capability (megawatts)

4,942

$\%$ of Total Summer Capability

74.4

Generation (gigawatthours)

29,406

Coal Deposits

Area (square miles)

Percent of State Land

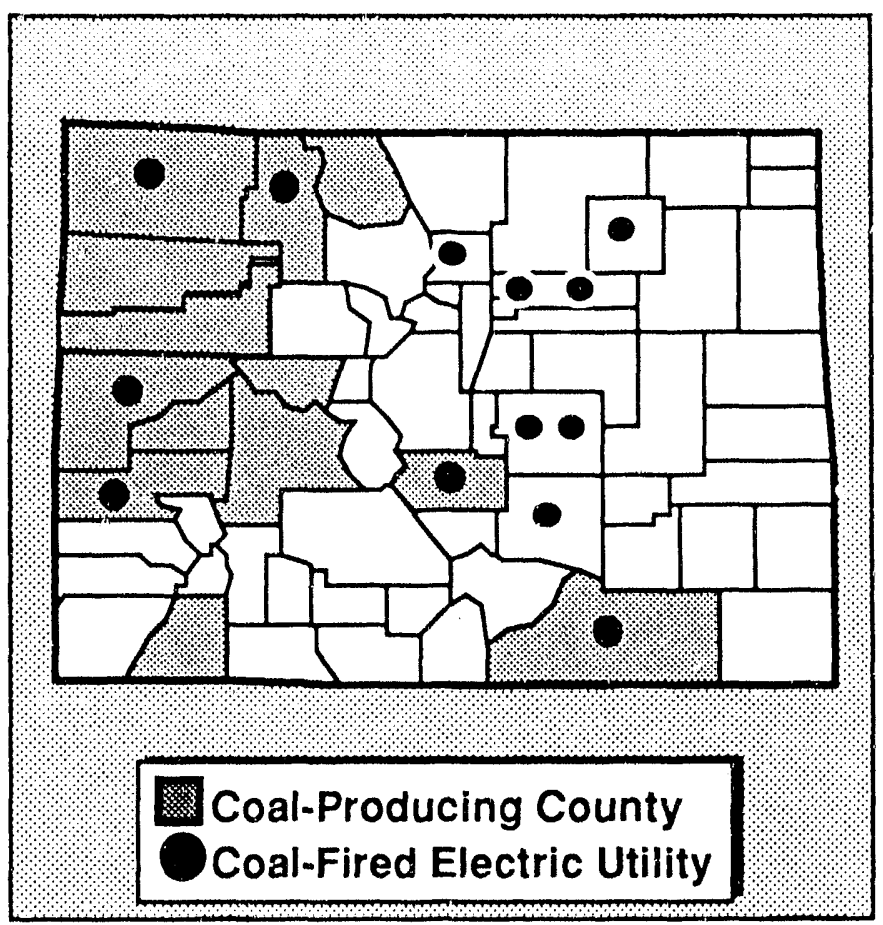

Table B11. Colorado Coal Facts

\begin{tabular}{|c|c|c|c|c|c|c|c|}
\hline & 1970 & 1975 & 1980 & 1985 & 1987 & 1988 & 1989 \\
\hline Production (Thousand Short Tons) ................... & 6,025 & 8,219 & 18,846 & 17,243 & 14,420 & 15,912 & 17,123 \\
\hline 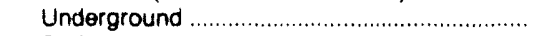 & 3,858 & 3,446 & 5,721 & 6,377 & 5,645 & 6,870 & 8,511 \\
\hline Surface & 2,167 & 4,773 & 13,125 & 10,865 & 8,775 & 9,042 & 8,612 \\
\hline Aver Mine Price (Dollars per Short Ton) & 5.85 & 16.53 & 19.89 & 24.65 & 23.58 & 23.09 & 23.64 \\
\hline Total Value of Production (Million Dollars) ... & 35 & 136 & 375 & 425 & 340 & 367 & 405 \\
\hline Percentege of U.S. Coal Production ............... & 1.0 & 1.3 & 2.3 & 2.0 & 1.6 & 1.7 & 1.7 \\
\hline Nattonal Rank (Production) .................................. & 12 & 14 & 12 & 14 & 15 & 15 & 15 \\
\hline $\begin{array}{l}\text { Total U.S. Coal Production } \\
\text { (Thousand Short Tons) }\end{array}$ & 612,661 & 654,641 & 829,700 & $\mathbf{8 8 3 , 6 3 8}$ & 918,762 & 950,265 & 980,729 \\
\hline Number of Mines' & 48 & 33 & 43 & 35 & 22 & 23 & 23 \\
\hline Underground & 40 & 18 & 26 & 23 & 9 & 15 & 16 \\
\hline Surtace & 8 & 15 & 17 & 12 & 13 & 8 & 7 \\
\hline Emptoyment ${ }^{2}$ & 1,273 & 1,850 & 3,911 & 2,551 & 1,785 & 2,103 & 2,019 \\
\hline 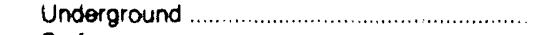 & 1,152 & 1,360 & 2,408 & 1,464 & 1,094 & $1,42.4$ & 1,490 \\
\hline Surface & 121 & 490 & 1,503 & 1,087 & 701 & 679 & 529 \\
\hline Coneumption (Thousend Short Tons) .............. & 5,136 & 8,210 & 11,981 & 15,242 & 15,007 & 15,860 & 16,393 \\
\hline Electric Utilities & 3,266 & 6,428 & 10,124 & 14,295 & 14,178 & 15,087 & 15,686 \\
\hline Coke Plants & 1,022 & 1,084 & (3) & & & & 0 \\
\hline Other Industrial Plants & (4) & (4) & (3) & $(4)$ & (4) & $(4)$ & $(4)$ \\
\hline Residential and Commercial .......................... & 4848 & i 698 & 31,857 & 948 & 4748 & 4773 & $\cdot 707$ \\
\hline
\end{tabular}

1 For 1980 , includes only mines that produced 10,000 or more tons.

2 Beginning in 1980 , includes only mines that produced 10,000 or more tons.

- Consumption data at Coke Plants, Other Industrial Plants and in Residential and Commercial Sector are combined to evoid disclosure of inơividual company data

- Consumption data at Other Industrial Plants and in Residential and Commercial Sector are combined to avoid disclosure of individual company data.

- Withheld to avoid disclosure of individual company data.

Sources: Energy Information Administration, Coal Production; Ouarterty Caal Report; Weokly Caal Production; Coal Data: A Fieference; Eloctric Power Monthly 1989; and Inventory of Power Plants in the United States 1989; Bureau of Mines, U.S. Department of Interior, Minerals Yearbook; Intormation Circular 8705: Historical Fuels and Energy Consumption Data, 1960-72, United States by States and Census Districts West of the Mississippi; and Georogical Survey Bulletin 1412: Coal Resources of the United States, January 1, 1974 


\section{Illinois}

\section{Coal Highlights}

Production

(thousand short tons)

Value

(million dollars)

Number of Employees

Consumption

(thousand short tons)

Coal-Fired Electric Generating Units

Number

Summer Capability (megawatts)

$\%$ of Total Summer Capability

Generation (gigawatthours)

Coal Deposits

Area (square miles)

Percent of State Land
59,267

10,003

32,374

14,934

37,700

67

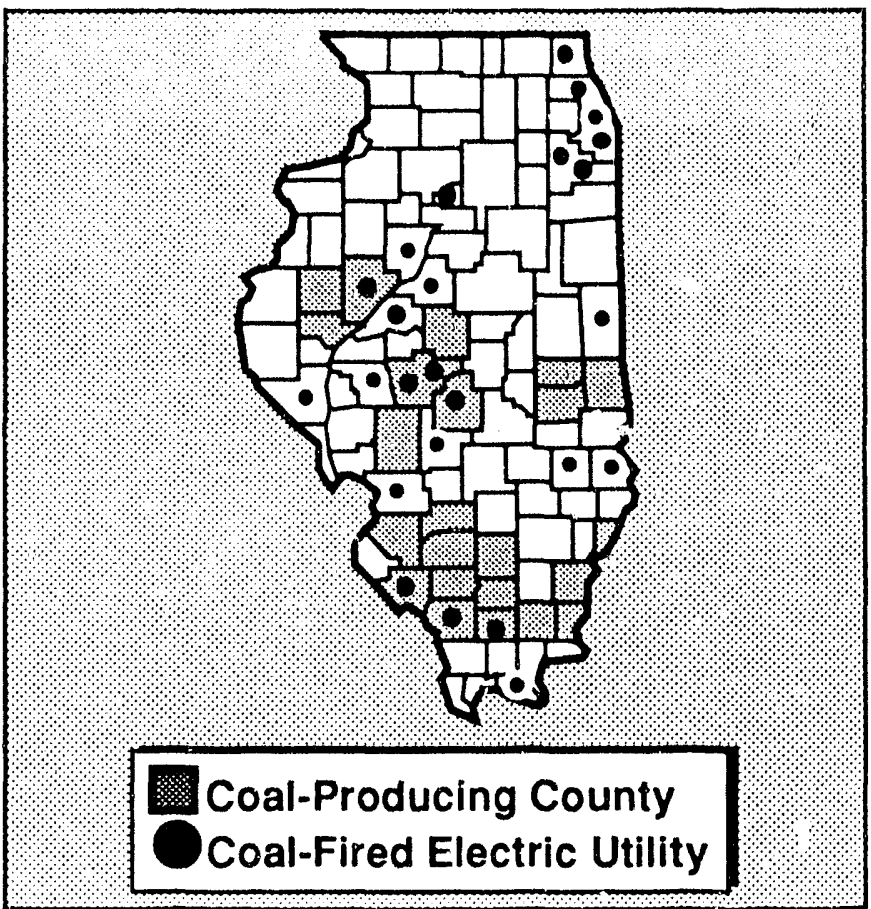

Table B12. Illinois Coal Facts

\begin{tabular}{|c|c|c|c|c|c|c|c|}
\hline & 1970 & 1975 & 1980 & 1985 & 1987 & 1998 & 1989 \\
\hline $\begin{array}{l}\text { Production (Thousand Short Tons) } \\
\text { Underground } \\
\text { Surtace }\end{array}$ & $\begin{array}{l}65,119 \\
32,093 \\
33,026\end{array}$ & $\begin{array}{l}59,537 \\
31,875 \\
27,661\end{array}$ & $\begin{array}{l}62,543 \\
34,969 \\
27,574\end{array}$ & $\begin{array}{l}59,201 \\
37,343 \\
21,858\end{array}$ & $\begin{array}{l}59,155 \\
37,521 \\
21,634\end{array}$ & $\begin{array}{l}58,504 \\
38,522 \\
20,072\end{array}$ & $\begin{array}{l}59,267 \\
39,337 \\
18,930\end{array}$ \\
\hline Averege Mine Price (Dollars per Short Ton) & 4.92 & 14.64 & 24.39 & 30.80 & 29.56 & 26.55 & 28.17 \\
\hline Total Value of Production (Million Dollars) ... & 320 & 872 & 1,525 & 1,823 & 1,749 & 1,673 & 1,670 \\
\hline Percentage of U.S. Cosl Production .............. & 10.6 & 9.1 & 7.5 & 6.7 & 6.4 & 6.2 & 6.0 \\
\hline National Rank (Production) .............................. & 4 & 4 & 5 & 5 & 5 & 5 & 5 \\
\hline $\begin{array}{l}\text { Total U.S. Cosl Production } \\
\text { (Thousand Short Tons) }\end{array}$ & 612,681 & 654,641 & 829,703 & $\mathbf{8 0 3 , 6 3 8}$ & 918,762 & 951,265 & 980,729 \\
\hline $\begin{array}{l}\text { Number of Mines' } \\
\text { Underground } \\
\text { Surface }\end{array}$ & $\begin{array}{l}59 \\
28 \\
31\end{array}$ & $\begin{array}{l}58 \\
21 \\
37\end{array}$ & $\begin{array}{l}59 \\
31 \\
28\end{array}$ & $\begin{array}{l}54 \\
34 \\
20\end{array}$ & $\begin{array}{l}51 \\
23 \\
22\end{array}$ & $\begin{array}{l}48 \\
28 \\
20\end{array}$ & $\begin{array}{l}48 \\
30 \\
18\end{array}$ \\
\hline $\begin{array}{l}\text { Emptoyment } \\
\text { Uriderground } \\
\text { Surtace }\end{array}$ & $\begin{array}{l}8,277 \\
6,057 \\
3,220\end{array}$ & $\begin{array}{r}12,850 \\
9,010 \\
3,840\end{array}$ & $\begin{array}{r}17,735 \\
12,610 \\
5,125\end{array}$ & $\begin{array}{r}13,858 \\
10,349 \\
3,509\end{array}$ & $\begin{array}{r}12,171 \\
8,932 \\
3,239\end{array}$ & $\begin{array}{r}10,022 \\
7,440 \\
2,582\end{array}$ & $\begin{array}{r}10,003 \\
8,084 \\
1,919\end{array}$ \\
\hline $\begin{array}{l}\text { Coneumption (Thousand Short Tons) } \\
\text { Electric Utilities } \\
\text { Coke Plants } \\
\text { Other Industrial Plants } \\
\text { Residential and Commercial }\end{array}$ & $\begin{array}{r}12,310 \\
29,448 \\
3,081 \\
3,181 \\
(3)\end{array}$ & $\begin{array}{r}11,946 \\
34,859 \\
3,104 \\
3,985 \\
(3)\end{array}$ & $\begin{array}{r}39,840 \\
34,610 \\
1,810 \\
3,265 \\
155\end{array}$ & $\begin{array}{r}37,601 \\
31,608 \\
2,358 \\
3,399 \\
236\end{array}$ & $\begin{array}{r}34,632 \\
28,894 \\
1,565 \\
3,900 \\
273\end{array}$ & $\begin{array}{r}32,97 ! \\
26,681 \\
1,678 \\
4,364 \\
256\end{array}$ & $\begin{array}{r}32,374 \\
25,758 \\
2,548 \\
3,770 \\
297\end{array}$ \\
\hline
\end{tabular}

1 For 1980 , includes only mines that produced 10,000 or more tons

2 Beginning in 1980, includes only mines that produced 10,000 or more tons.

- Consumption data at Other Industrial Plants and in Residential and Commercial Sector are combined to avord disclosure of individual company data Note: Total may not equal sum of components becausf of independent rounding.

Sources: Energy Intormation Administration, Caal Production; Ouarterty Coal Report; Weekly Coc! Production; Coal Date A Reference; Electric Power Monthly 1980; ant Inventory of Power Plants in the United States 1989; Bureau of Mines, U.S. Department of Interior, Minerals Yearbook: Informetion Circular 8705: Histoncal Fuels and Energy Consumption Data, 1960-72, Unted States by States and Census Districts West of the Mississippi; and Gea logical Survey Bulletin 1412: Coal Resources of the United States, January 1. 1974. 


\section{Coal Highlights}

Production

(thousand short tons)

33,641

Value

(million dollars)

Number of Employees

3,684

Consumption

(thousand short tons)

58,203

Coal-Fired Electric Generating Units

Number

Summer Capability (megawatts)

\% of Total Summer Capability

19,579

Generation (gigawatthours)

94.8

87,330

Coal Deposits

Area (square miles)

Percent of State Land

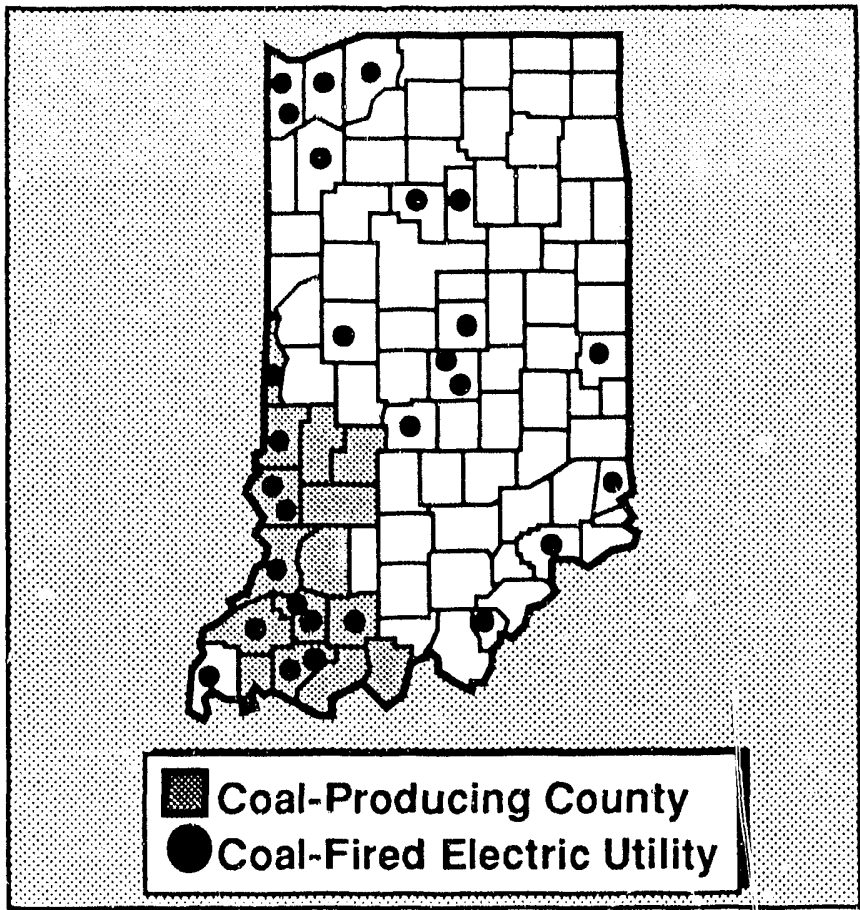

Table B13. Indiana Coal Facts

\begin{tabular}{|c|c|c|c|c|c|c|c|}
\hline & 1970 & 1975 & 1980 & 1985 & 1987 & 1903 & 1989 \\
\hline Production (Thousand Short Tons) ...................... & 22,263 & 25,124 & 30,873 & 33,316 & 34,208 & 31,271 & 33,641 \\
\hline 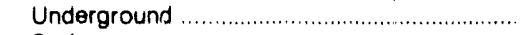 & 2,094 & 188 & 688 & 2,053 & 2,447 & 2,409 & 2,519 \\
\hline Surface & 20,169 & 24,935 & 30,185 & 31,262 & 31,761 & 28,862 & 31,122 \\
\hline Average Mine Price (Dollars per Short Ton) & 4.60 & 11.15 & 20.72 & 26.54 & 24.57 & 24.28 & 23.55 \\
\hline Total Value of Production (Million Dollars) ... & 102 & 280 & 640 & 884 & 840 & 760 & 792 \\
\hline Percentage of U.S. Coal Production ................ & 3.6 & 3.8 & 3.7 & 3.8 & 3.7 & 3.3 & 3.4 \\
\hline National Rank (Production) ................................... & 7 & 7 & 8 & 9 & 10 & 10 & 10 \\
\hline $\begin{array}{l}\text { Total U.S. Coal Production } \\
\text { (Thousand Short Tons) }\end{array}$ & 612,661 & 654,641 & 829,700 & 883,638 & 918,762 & 950,265 & 980,729 \\
\hline Number of Mines' & 38 & 62 & 87 & 70 & 67 & 73 & 60 \\
\hline $\begin{array}{l}\text { Underground } \\
\text { Surtace }\end{array}$ & $\begin{array}{r}6 \\
32\end{array}$ & $\begin{array}{r}2 \\
60\end{array}$ & $\begin{array}{r}4 \\
83\end{array}$ & $\begin{array}{r}4 \\
66\end{array}$ & $\begin{array}{r}5 \\
62\end{array}$ & $\begin{array}{r}5 \\
68\end{array}$ & $\begin{array}{r}6 \\
54\end{array}$ \\
\hline Employment ${ }^{2} \ldots$ & 2,275 & 3,040 & 5,315 & 5,187 & 3,992 & 4,108 & 3,684 \\
\hline 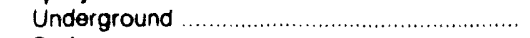 & 487 & 80 & 133 & 455 & 489 & 402 & 521 \\
\hline Surface & 1.788 & 2,960 & 5,182 & 4,732 & 3,503 & 3.706 & 3,163 \\
\hline Coneumption (Thousand Short Tons) ............. & 42,385 & 46,928 & 50,485 & 53,291 & 52,335 & 56,762 & 58,203 \\
\hline Electric Utilities & 22,760 & 28,720 & 33,664 & 38,310 & 36,987 & 40,060 & 42,378 \\
\hline Coke Plants & 12,673 & 14,078 & 11,989 & 9,338 & 9,791 & 11,287 & 10,568 \\
\hline Other Industrial Plants & 36,952 & 34,130 & 4,610 & 5.119 & 5,016 & 4,784 & 4,738 \\
\hline Residential and Commercial ............................... & (3) & (3) & 222 & 524 & 541 & 631 & 518 \\
\hline
\end{tabular}

1 For 1980 , includes only mines that produced 10,000 or more tons

2 Beginning in 1980, includes only mines that produced 10,000 or more tons

3 Consumption data at Other Industrial Plants and in Residantial and Commercial Sectol are combined to avold disclosure of individual company data. Note: Tctal may not equal sum of components because of independent rounding.

Sources: Energy Information Administration, Coal Production; Quartenty Coal Relort; Weekly Coal Production; Coal Data: A Relerence; Electric Power Monthly 1989; and Inventory of Power Plants in the United States 1989: Bureau if Mines, U.S. Department of Interior, Minera/s Yeartook; Intorme tron Circular 8705: Histoncal Fuels and Energy Consumption Data, 1360.72, United States by States and Census Districts West of the Mississippl; and Geo. logical Survey Bulletin 1412: Coal Resources of the United States, January 1, 1974. 


\section{Coal Highlights}

Production

(thousand short tons)

Value

(million dollars)

Number of Employees

w

Consumption

(thousand short tons)

17,103

Coal-Fired Electric Generating Units

Number

Summer Capability (megawatts)

$\%$ of Total Summer Capability

Generation (gigawatthours)

23,724

Coal Deposits

Area (square miles)

Percent of State Land
20,000

36

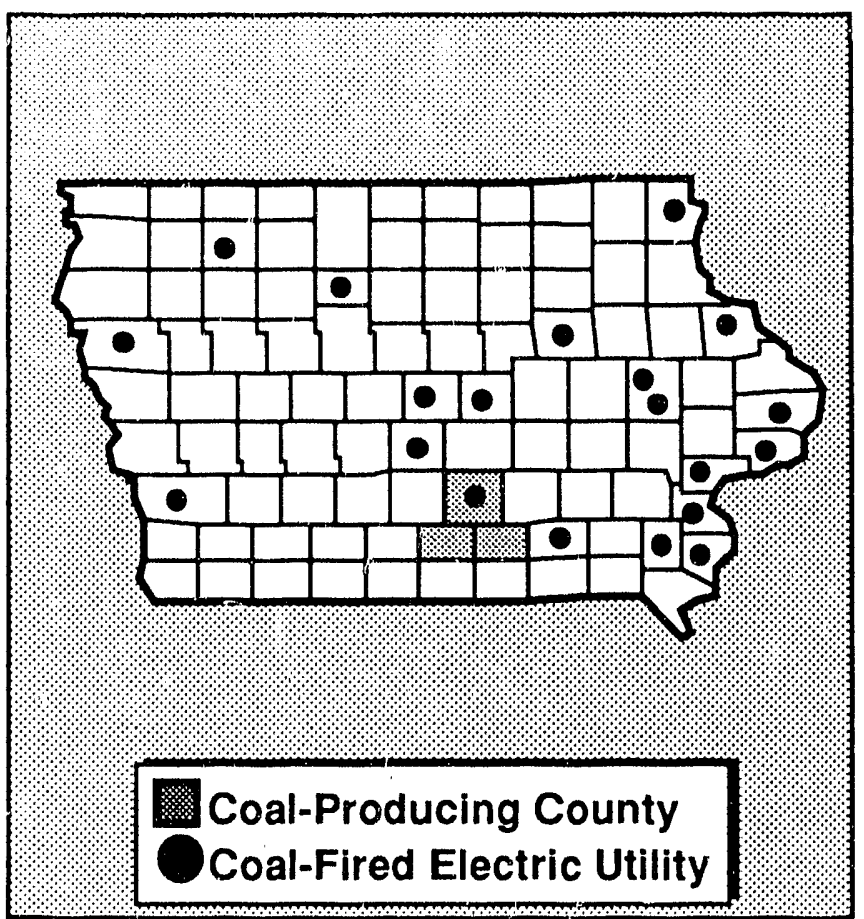

Table B14. lowa Coal Facts

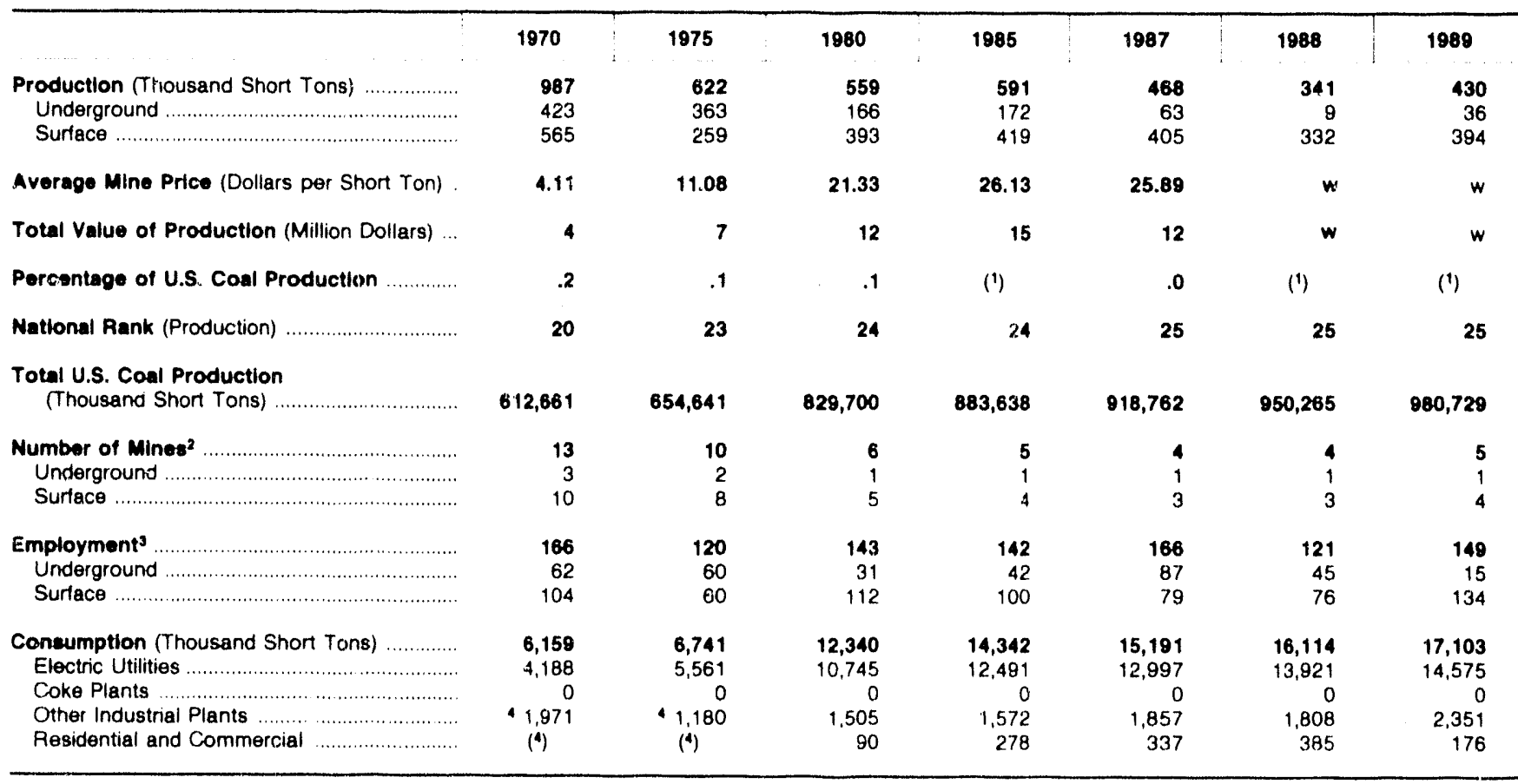

1 Less than 0.05 percent.

2 For 1980 , includes only mines that produced 10,000 or more tons

3 Beginning in 1980, includes only mines that produced 10,000 or more tons

- Consumption data at Other Industrial Plants and in Residential and Commercial Sector are combined to avoid disclosure of individual company data Note: Total may not enual sum of components because of independent rounding.

Sources: Energy Information Administration, Coal Production; Quarterty Caal Report; Weekly Coal Production: Coal Data: A Reterence; Electric Power Monthly 1989; and Inventory of Power Plants in the United Staies 1989; Bureau of Mines. U.S. Department of interior, Minera/s Yeartook: Information Circular 8705: Historical Fuals and Energy Consumption Date, 1960-72, United States by States and Census Districts West of the Mississippi; and Geo. logical Sunvey Bulletin 1412: Coal Resources of the United States, January 1, 1974. 


\section{Coal Highlights}

Production

(thousand short tons)

856

Value

(million dollars)

Number of Employees

Consumption

(thousand short tons)

14,963

Coal-Fired Electric Generating Units

Number

Summer Capability (megawatts)

5,064

$\%$ of Total Summer Capability

Generation (gigawatthours)

22,907

Coal Deposits

Area (square miles)

Percent of State Land

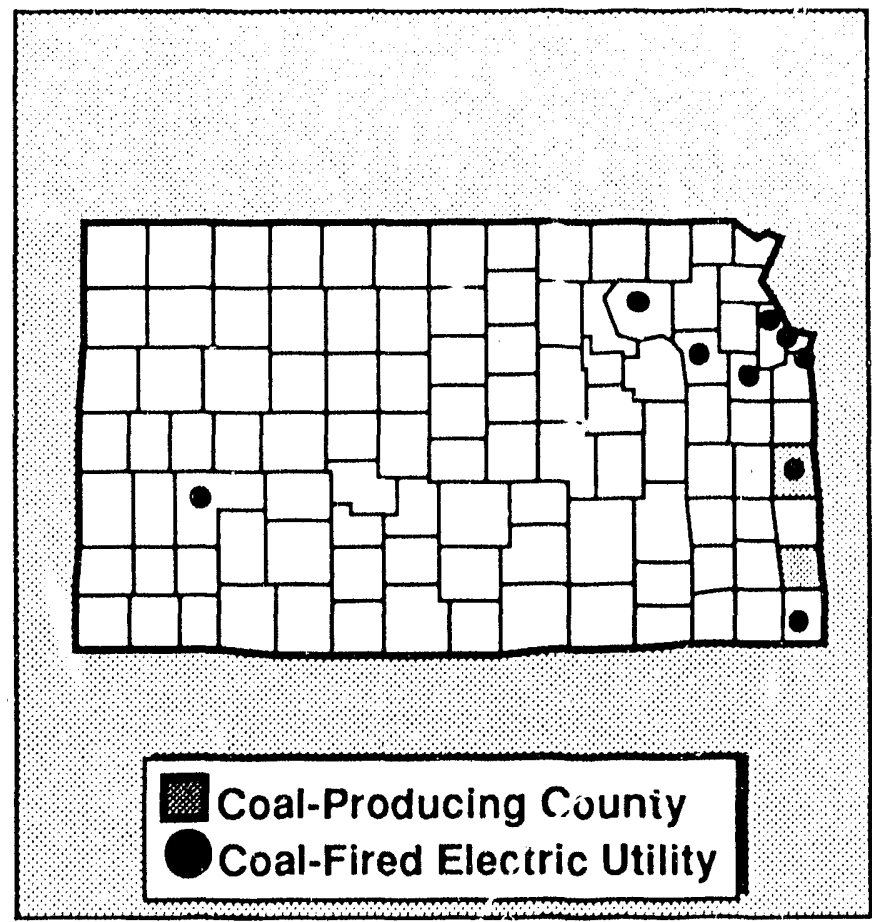

Table B15. Kansas Coal Facts

\begin{tabular}{|c|c|c|c|c|c|c|c|}
\hline & 1970 & 1975 & 1980 & 1985 & 1987 & 1988 & 1989 \\
\hline Production (Thousand Short Tons) ..................... & 1,627 & 470 & 342 & 904 & 2,021 & 737 & 856 \\
\hline 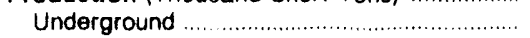 & 0 & 0 & 0 & 0 & 0 & 0 & 0 \\
\hline Surtace & 1,627 & 478 & 842 & 984 & 2,021 & 737 & 856 \\
\hline Avereog Mine Price (Dollars per Short Ton) & 5.59 & 19.78 & 26.54 & 26.00 & 24.54 & 30.59 & 27.00 \\
\hline Total Value of Production (Million Collars) & $\theta$ & $\boldsymbol{\theta}$ & 22 & 26 & 50 & 22 & 23 \\
\hline Percentage of U.S. Coal Production ............. & .3 & .1 & .1 & .1 & .2 & .1 & .1 \\
\hline National Rank (Production). & 18 & 26 & 22 & 23 & 23 & 24 & 24 \\
\hline $\begin{array}{l}\text { Total U.S. Coal Production } \\
\text { (Thousand Short Tons) }\end{array}$ & 612,681 & 654,641 & 829,700 & 883,838 & 818,762 & 950,265 & 990,729 \\
\hline Number of mines'. & 5 & 4 & 8 & 5 & 5 & 6 & 5 \\
\hline Underground & 0 & 0 & 0 & 0 & 0 & 0 & 0 \\
\hline Surtace & 5 & 4 & 8 & 5 & 5 & 6 & 5 \\
\hline Emptoyment ${ }^{2}$ & 235 & 130 & 274 & 228 & 247 & 268 & 165 \\
\hline Underground & 0 & 0 & 0 & 0 & 0 & 0 & 0 \\
\hline Surtace & 235 & 130 & 274 & 228 & 247 & 238 & 165 \\
\hline Coneumption (Trrousand Short Tons) & 1,974 & 3,333 & 10,371 & 14,715 & 15,194 & 14,051 & 14,063 \\
\hline 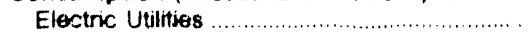 & 1.589 & 3,220 & 10.035 & 14,351 & 14,842 & 14,742 & 14,575 \\
\hline 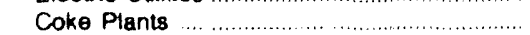 & 0 & 0 & 0 & 0 & 0 & 0 & 0 \\
\hline Other Industral Plants & 320 & 3113 & 331 & 363 & $25 ?$ & 208 & 183 \\
\hline Residential und Commercial & 55 & (3) & 5 & 1 & 1 & 1 & 0 \\
\hline
\end{tabular}

1 For 1980 , includes only mines that produced 10,000 or more lons

2 Beginning in 1980, includes only mines that produced 10,000 or more tons

- Consumption data at Other Industrial Plants and in Residential and Commercial Sector are combined to avoid disclosure of individual company data Note: Total may not equal sum of components because of independent rounding.

Sources: Energy Information Administration, Coal Production; Quarterty Casl Report; Weokly Coal Production, Coal Dala. A Reference; Electric Power Monthly 1989; and Irventory of Power Plants in the United States 1989; Bureau of Mines. U.S. Department of Interior, Minera/s Yeartiook; Intorma. tion Circular 8705. Histoncal Fuels and Energy Consumption Data, 1960.72. United States by Stales and Census Distncts West of the Mississippl; and Geo. logical Survey Bulletin 1412: Coal Resources of the United Stales, January 1, 1974. 


\section{Kentucky}

\section{Coal Highlights}

Production

(thousand short tons)

167,389

Value

(million dollars)

Number of Employees

30,658

Consumption

(thousand short tons)

32,792

Coal-Fired Electric Generating Units

Number

Summer Capability (megawatts)

$\%$ of 'Total Summer Capability

13,818

92.0

Generation (gigawatthours)

66,214

Coal Deposits

Area (square miles)

Percent of State Land

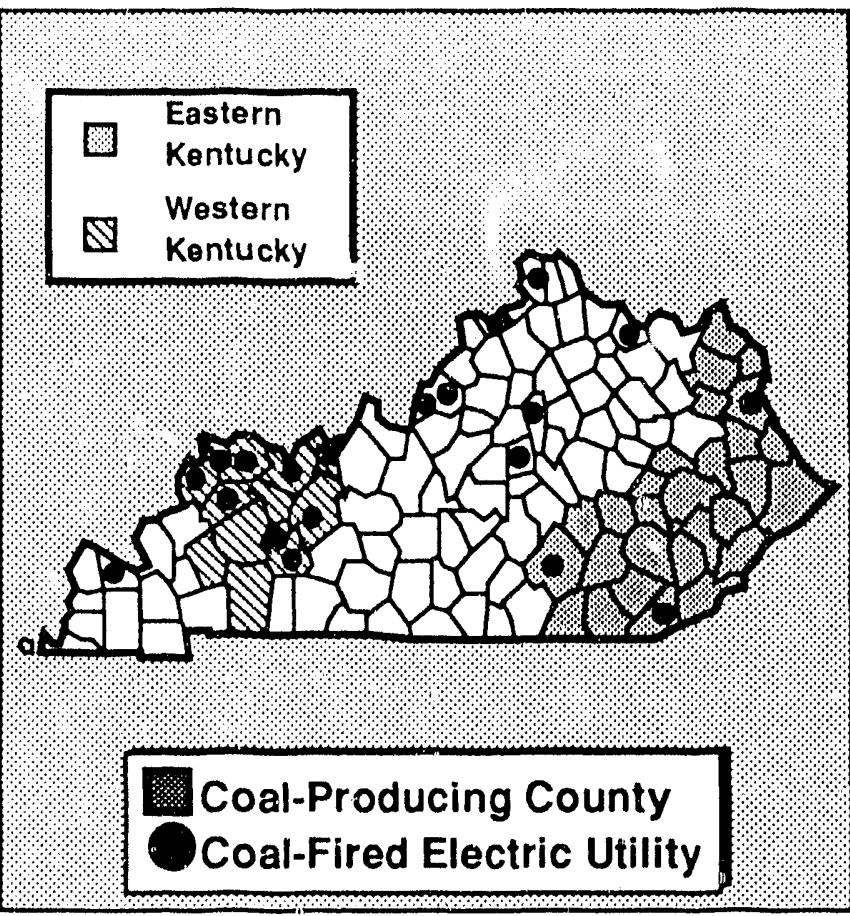

Table B16. Kentucky Coal Facts

\begin{tabular}{|c|c|c|c|c|c|c|c|}
\hline & 1970 & 1975 & 1980 & 1985 & 1087 & 1988 & 1989 \\
\hline $\begin{array}{l}\text { Production (Thousand Short Tons) } \\
\text { Underground }\end{array}$ & $\begin{array}{r}125,305 \\
62,610 \\
62,695\end{array}$ & $\begin{array}{r}143,613 \\
65,632 \\
77,981\end{array}$ & $\begin{array}{r}150,144 \\
79,162 \\
70,982\end{array}$ & $\begin{array}{r}152,272 \\
81,236 \\
71,036\end{array}$ & $\begin{array}{r}165,192 \\
92,882 \\
72,310\end{array}$ & $\begin{array}{r}157,852 \\
63,131 \\
94,720\end{array}$ & $\begin{array}{r}167,369 \\
99,067 \\
68,322\end{array}$ \\
\hline Average Mine Price (Dollars per Short Ton) & 5.68 & 17.40 & 27.62 & 28.24 & 26.15 & 26.20 & 24.97 \\
\hline Total Value of Production (Million Dollars) ... & 712 & 2,400 & 4,147 & 4,300 & 4,320 & 4,136 & 4,180 \\
\hline Percentage of U.S. Coal Production .............. & 20.5 & 21.9 & 18.1 & 17.2 & 18.0 & 16.8 & 17.1 \\
\hline National Rank (Production) ............................... & 2 & 1 & 1 & 1 & 1 & 2 & 2 \\
\hline $\begin{array}{l}\text { Total U.S. Coal Production } \\
\text { (Thousand Short Tons) }\end{array}$ & 612,661 & 654,841 & 829,700 & 883,638 & 918,762 & 950,265 & 980,729 \\
\hline $\begin{array}{l}\text { Number of Mines' } \\
\text { Underground } \\
\text { Surface }\end{array}$ & $\begin{array}{r}1,721 \\
1,104 \\
617\end{array}$ & $\begin{array}{r}2,401 \\
896 \\
1,505\end{array}$ & $\begin{array}{r}1,150 \\
647 \\
503\end{array}$ & $\begin{array}{r}1,858 \\
921 \\
937\end{array}$ & $\begin{array}{r}1,428 \\
816 \\
612\end{array}$ & $\begin{array}{r}1,230 \\
738 \\
492\end{array}$ & $\begin{array}{r}1,099 \\
670 \\
429\end{array}$ \\
\hline $\begin{array}{l}\text { Employment } \\
\text { Underground } \\
\text { Surlace }\end{array}$ & $\begin{array}{r}25,691 \\
18,843 \\
6,848\end{array}$ & $\begin{array}{l}36,070 \\
22,200 \\
13,870\end{array}$ & $\begin{array}{l}46,395 \\
30,581 \\
15,814\end{array}$ & $\begin{array}{l}36,814 \\
22,877 \\
13,837\end{array}$ & $\begin{array}{l}32,580 \\
21,505 \\
11,085\end{array}$ & $\begin{array}{l}30,559 \\
20,473 \\
10,086\end{array}$ & $\begin{array}{r}30,658 \\
20,752 \\
9,904\end{array}$ \\
\hline $\begin{array}{l}\text { Conaumption (Thousand Short Tons) } \\
\text { Electric Utilities } \\
\text { Coke Plants .................. } \\
\text { Other Industrial Plants } \\
\text { Residential and Commercial }\end{array}$ & $\begin{array}{r}23,672 \\
19,103 \\
1,586 \\
42,983 \\
(4)\end{array}$ & $\begin{array}{r}28,480 \\
25,717 \\
1,253 \\
41,510 \\
(4)\end{array}$ & $\begin{array}{r}27,524 \\
24,383 \\
905 \\
1,969 \\
267\end{array}$ & $\begin{array}{l}30,990 \\
27,085 \\
(3) \\
2,510 \\
31,396\end{array}$ & $\begin{array}{l}32,023 \\
28,569 \\
(3) \\
1,896 \\
1,584\end{array}$ & $\begin{array}{l}35,176 \\
31,818 \\
(3) \\
1,702 \\
31,656\end{array}$ & $\begin{array}{l}32,792 \\
28,109 \\
(3) \\
2,168 \\
3 \\
1,515\end{array}$ \\
\hline $\begin{array}{l}1 \text { For } 1980 \text {, includes only mines that produc } \\
2 \text { Beginning in } 1980 \text {, includes only mines the } \\
\text { 3 Consumption data at Coke Plants and in } \\
1 \text { Consumption data at Other Industrial Plan } \\
\text { Note: Total may not equal sum of compo } \\
\text { Sources: Energy Information Administrat } \\
\text { Power. Monthly } 1989 \text {; and Inventory of Power } \\
\text { ton Circular } 8705 \text {. Histoncal Fuels and Energy } \\
\text { logrcal Survey Bulletin 1412: Coal Resources }\end{array}$ & Coal & on: Qut & $\begin{array}{l}\text { are com } \\
\text { cial Sect } \\
\text { inding. } \\
\text { Coal Rep } \\
\text { Bureau of } \\
\text { d Stales } \\
\text { 74. }\end{array}$ & $\begin{array}{l}\text { ekfly Co } \\
\text { U.S. D }\end{array}$ & $\begin{array}{l}\text { ure of in } \\
\text { pid disclo } \\
\text { duction: } \\
\text { ont of int }\end{array}$ & $\begin{array}{l}\text { Cumpan } \\
\text { individu }\end{array}$ & $\begin{array}{l}\text { pany data. } \\
\text { ce: Eloctric } \\
\text { ok; Intorma. } \\
\text { pi; and Geo- }\end{array}$ \\
\hline
\end{tabular}




\section{L.ouisiana}

\section{Coal Highlights}

Production

(thousand short tons)

Value

(million dollars)

Number of Employees

w

Consumption

(thousand short tons)

12,471

Coal-Fired Electric Generating Units

Number

Summer Capability (megawatts)

$\%$ of Total Summer Capability

Generation (gigawatthours)

\section{Coal Deposits}

Area (square miles)

Percent of State Land

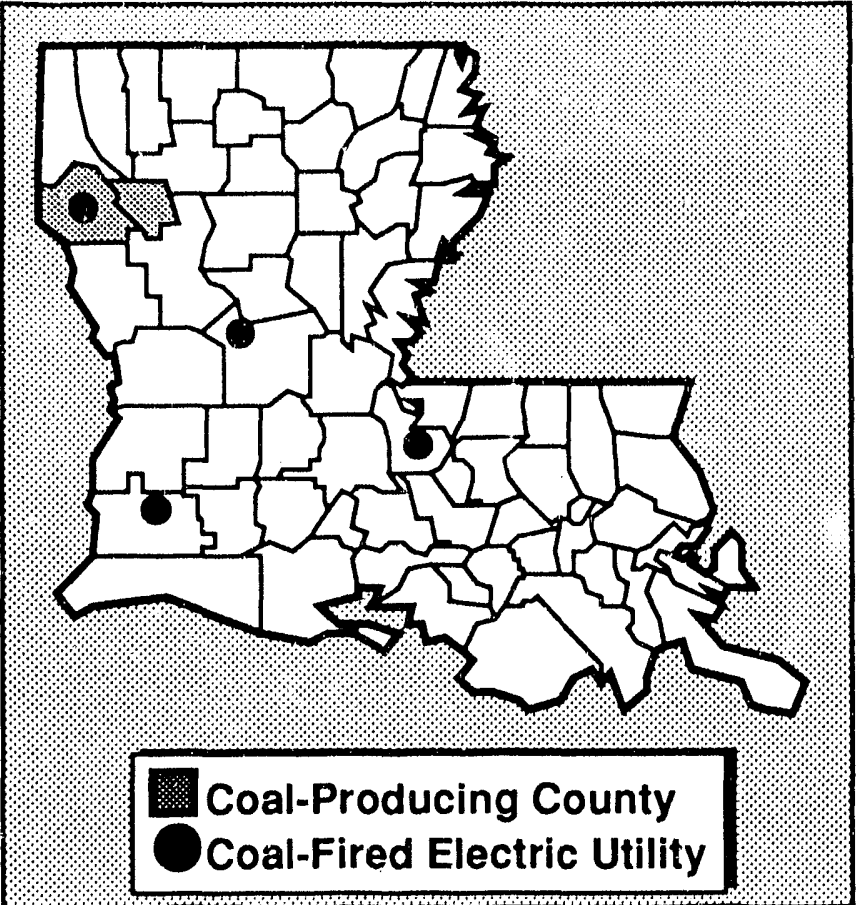

Table B17. Louisiana Coal Facts

\begin{tabular}{|c|c|c|c|c|c|c|c|}
\hline$\ldots$ & 1970 & 1975 & 1980 & 1985 & 1987 & 1988 & 1989 \\
\hline Production (Thousand Short Tons) .................... & 0 & 0 & 0 & 207 & 2,751 & 2,889 & 2,983 \\
\hline 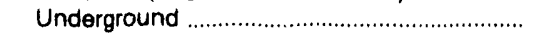 & 0 & 0 & 0 & 0 & 0 & 0 & 0 \\
\hline 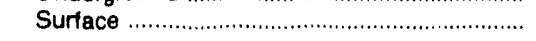 & 0 & 0 & 0 & 207 & 2,751 & 2,889 & 2,983 \\
\hline Average Mine Price (Dollars per Short Ton) & .00 & .00 & .00 & 6.05 & w & w & $\mathbf{w}$ \\
\hline Total Value of Production (Million Dollars) ... & 0 & 0 & 0 & 1 & w & w & $\mathbf{w}$ \\
\hline Percentage of U.S. Coal Production ................. & .0 & .0 & .0 & (1) & 3 & .3 & .3 \\
\hline Natlonal Rank (Production) ................................. & 0 & 0 & 0 & 25 & 22 & 21 & 21 \\
\hline $\begin{array}{l}\text { Total U.S. Coal Production } \\
\text { (Thousand Short Tons) }\end{array}$ & 612,661 & 654,641 & 829,700 & 883,638 & 918,762 & 950,265 & 980,729 \\
\hline 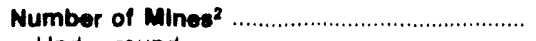 & o & 0 & 0 & 1 & 1 & 1 & 2 \\
\hline Underground & 0 & 0 & 0 & 0 & 0 & 0 & 0 \\
\hline Surface & 0 & 0 & 0 & 1 & 1 & 1 & 2 \\
\hline 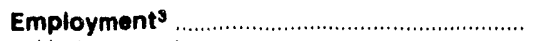 & 0 & 0 & 0 & 72 & 81 & 78 & 69 \\
\hline 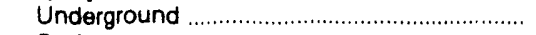 & 0 & 0 & 0 & 0 & 0 & 0 & 0 \\
\hline 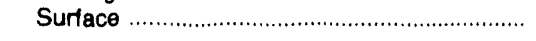 & 0 & 0 & 0 & 72 & 81 & 78 & 69 \\
\hline Coneumption (Thousand Short Tons) .............. & 2,317 & 0 & 111 & 9,217 & 10,381 & 12,848 & 12,471 \\
\hline 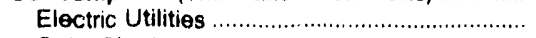 & w & 0 & 0 & 8,760 & 10,029 & 12,301 & 11,770 \\
\hline Coke Plants & w & 0 & 0 & 0 & 0 & 0 & 0 \\
\hline Other Industrial Plants .......................................... & w & 0 & 107 & 4457 & 4362 & a 547 & 4701 \\
\hline Residential and Commercial ............................. & $\mathbf{w}$ & 0 & 4 & (4) & $(4)$ & $\left({ }^{4}\right)$ & $(4)$ \\
\hline
\end{tabular}

1 Less than 0.05 percent.

2 For 1980 , includes only mines that produced 10,000 or more tons

$\checkmark$ Includes only mines that produced 10,000 or more tons.

- Consumption data at Other Industrial Plants and in Residential and Commercial Sector are combined to avoid disclosure of individual company data.

- Withheld to avoid disclosure of individual company data.

Note: Total may not equal sum of components because of independent rounding.

Sources: Energy information Administration, Coal f'oduction; Quarterly Coal Report; Weekly Coal Production; Coal Data: A Reference; Electric Power Monthly 1989; and Inventory of Power Plants in the United States 1989; Bureau of Mines, U.S. Department of Interior, Minerals Yeartoon; Information Circular 8705: Historical Fuels and Energy Consumption Data, 1960-72, United State's by States and Census Districts West of the Mississippi; and Geological Survey Bulletin 1412: Coal Resources of the United States, January 1, 1974. 


\section{Coal Highlights}

Production

(thousand short tons)

Value

(million dollars)

Number of Employees

Consumption

(thousand short tons)

Coal-Fired Electric Generating Units

Number

Summer Capability (megawatts)

$\%$ of Total Summer Capability

Generation (gigawatthours)

3,376

\section{Coal Deposits}

Area (square miles)

Percent of State Land

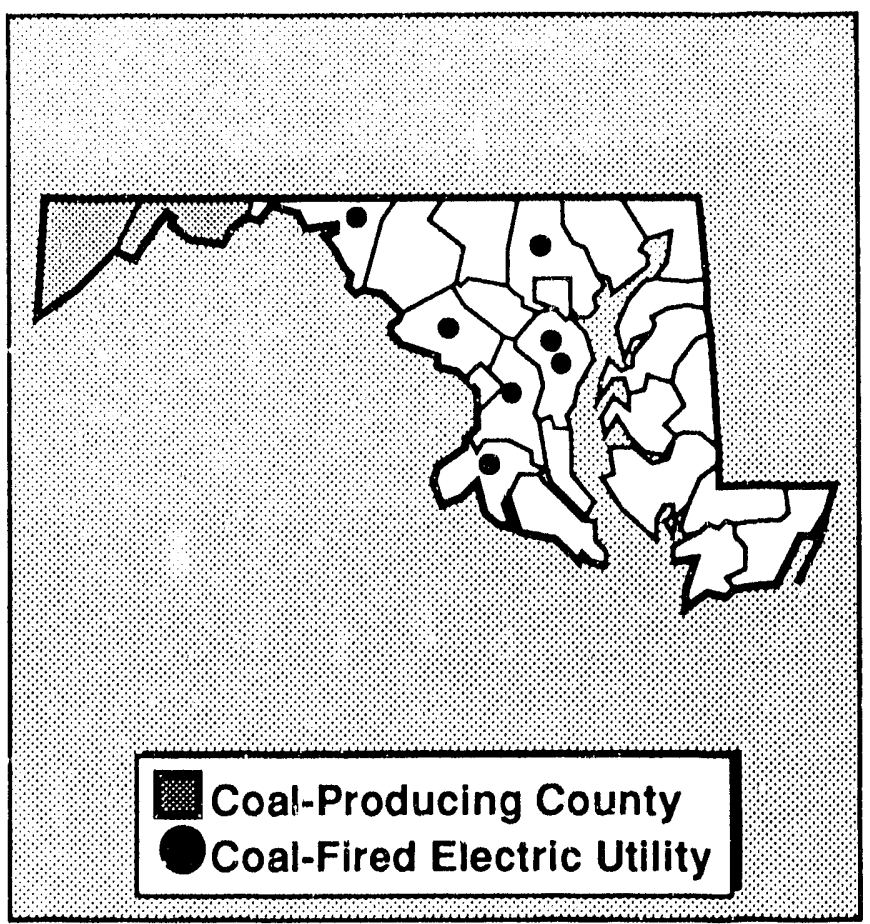

Table B18. Maryland Coal Facts

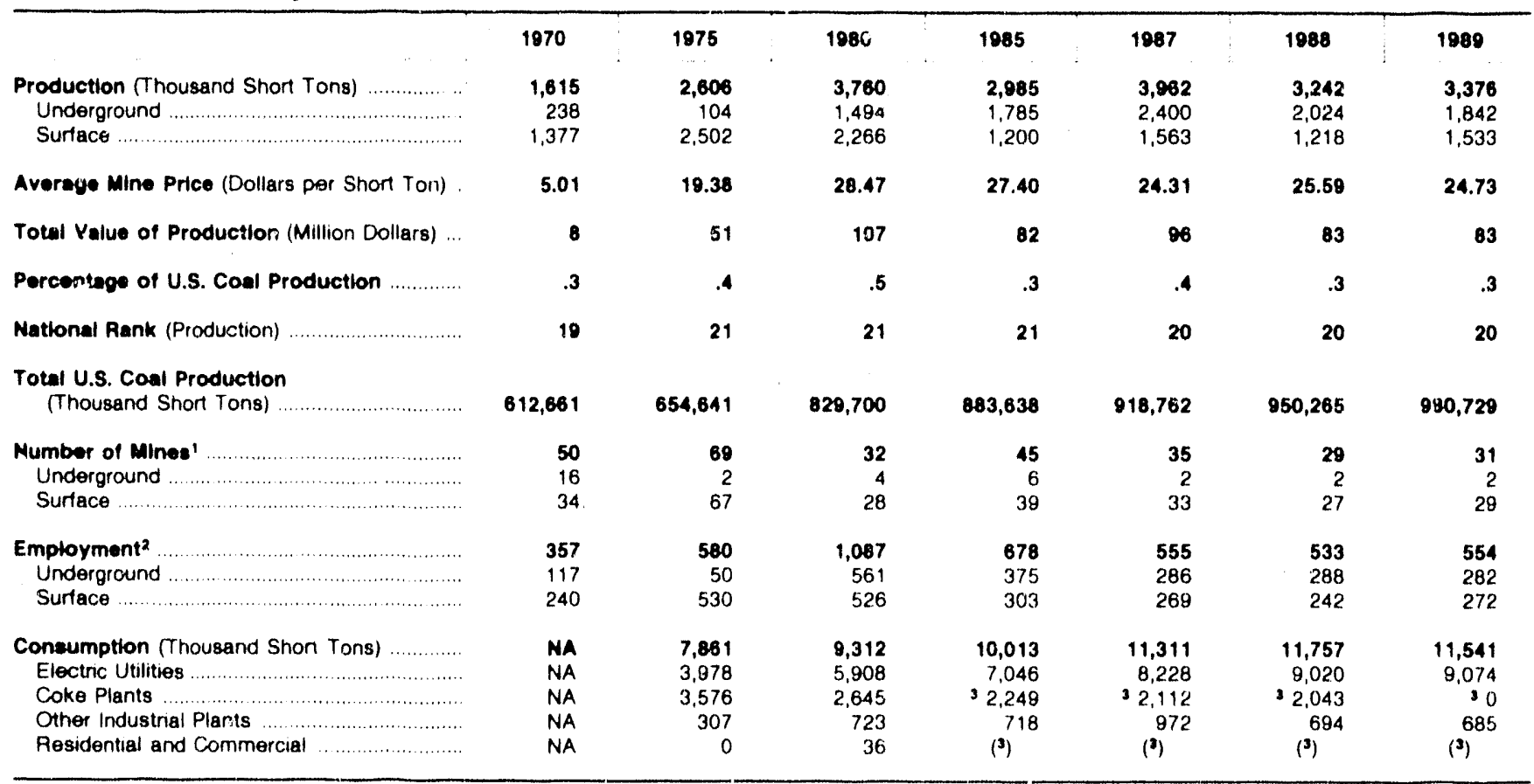

1 For 1880 , includes only mines that produced 10,000 or more tons.

- Beginning in 1980, includes only mines that produced 10,000 or more tons

3 Consumption data at Coke Plants and in Residential and Commercial Sector ares combined to avord disciosure of individual company data.

ma Not Available.

Note: Total may not equal sum of components because of independent rounding

Sources: Energy Information Administration, Caal Production; Quarterty Coal Report; Weekly Coal Production; Coal Data: A Relerence; Electric Power Monthly 1989; and Inventory of Power Plants in the United States 1989; Bureau of Mintis, US Department of interior, Minerals Yearbook: Intorma. tion Circular 8705: Historical Fuels and Energy Consumption Data, 1960-72, United States by Siates ard Census Districts West of the Mississippi; and Geotogical Survey Bulletin 1412: Coal Resources of the United States, January 1, 1974. 


\section{Coal Highlights}

Production

(thousand short tons)

3,378

Value

(million dollars)

Number of Employees

w

Consumption

(thousand short tons)

Coal-Fired Electric Generating Units

Number

Summer Capability (megawatts)

$\%$ of Total Summer Capability

Generation (gigawatthours)

26,297

10,649

70.5

49,754

Coal Deposits

Area (square miles)

24,700

Percent of State Land

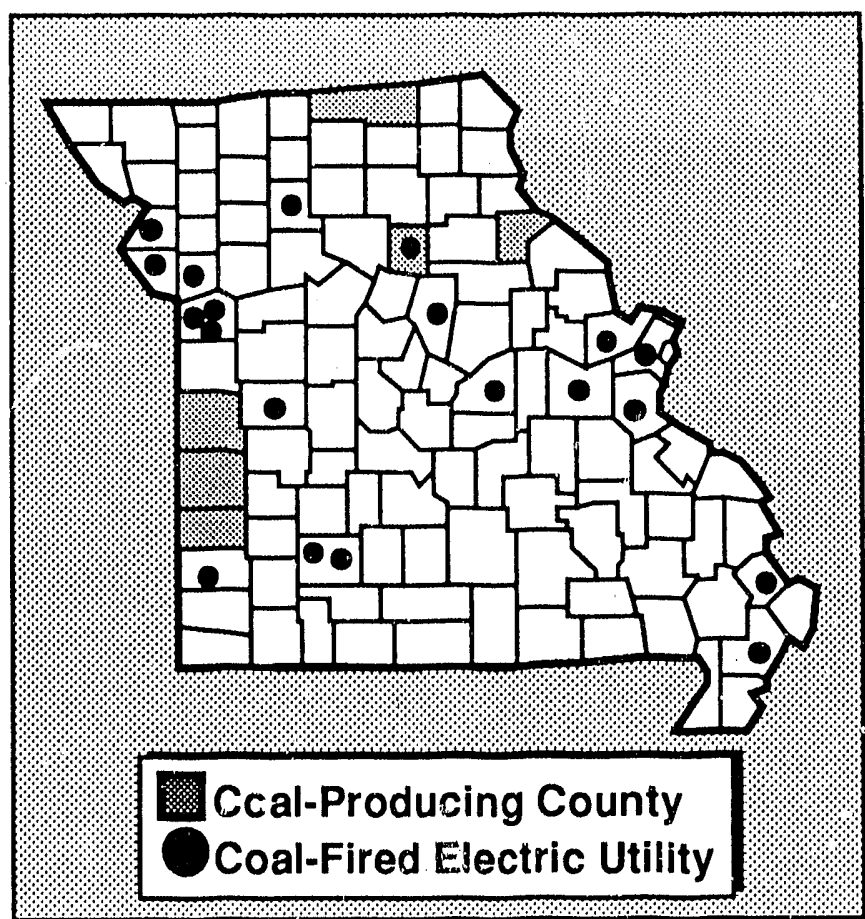

Table B19. Missouri Coal Facts

\begin{tabular}{|c|c|c|c|c|c|c|c|}
\hline & 1970 & 1975 & 1880 & 1985 & 1987 & 1988 & 1989 \\
\hline 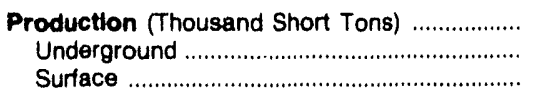 & $\begin{array}{r}4,447 \\
0 \\
4,447\end{array}$ & $\begin{array}{r}5,638 \\
0 \\
5,638\end{array}$ & $\begin{array}{r}5,503 \\
0 \\
5,503\end{array}$ & $\begin{array}{r}5,571 \\
5 \\
5,566\end{array}$ & $\begin{array}{r}4,282 \\
0 \\
4,292\end{array}$ & $\begin{array}{r}4,169 \\
0 \\
4,169\end{array}$ & $\begin{array}{r}3,378 \\
0 \\
3,378\end{array}$ \\
\hline Average Mine Price (Dollars per Short Ton). & 4.39 & 8.52 & 20.50 & 27.08 & 28.82 & 27.06 & $\mathbf{w}$ \\
\hline Total Value of Production (Million Dollars) ... & 20 & 48 & 113 & 151 & 124 & 113 & $w$ \\
\hline Percentage of U.S. Coal Production .............. & .7 & .9 & .7 & .6 & .5 & .4 & .3 \\
\hline National Rank (Production) .................................... & 15 & 18 & 18 & 18 & 19 & 18 & 19 \\
\hline $\begin{array}{l}\text { Total U.S. Coal Production } \\
\text { (Thousand Short Tons) }\end{array}$ & 612,661 & 654,641 & 829,700 & 883,638 & 918,762 & 950,265 & 980,729 \\
\hline 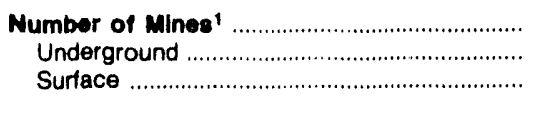 & $\begin{array}{l}9 \\
0 \\
9\end{array}$ & $\begin{array}{r}13 \\
0 \\
13\end{array}$ & $\begin{array}{r}13 \\
0 \\
13\end{array}$ & $\begin{array}{r}15 \\
1 \\
14\end{array}$ & $\begin{array}{r}12 \\
0 \\
12\end{array}$ & $\begin{array}{r}10 \\
0 \\
10\end{array}$ & $\begin{array}{l}8 \\
0 \\
9\end{array}$ \\
\hline 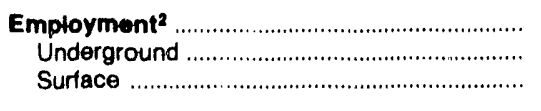 & $\begin{array}{r}480 \\
0 \\
480\end{array}$ & $\begin{array}{r}880 \\
0 \\
890\end{array}$ & $\begin{array}{r}1,093 \\
0 \\
1,093\end{array}$ & $\begin{array}{r}1,108 \\
0 \\
1,108\end{array}$ & $\begin{array}{r}814 \\
0 \\
814\end{array}$ & $\begin{array}{r}671 \\
0 \\
671\end{array}$ & $\begin{array}{r}428 \\
0 \\
428\end{array}$ \\
\hline 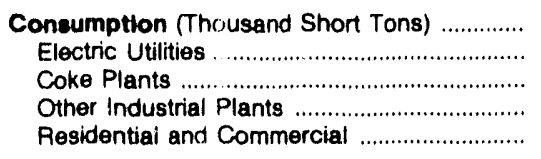 & $\begin{array}{r}13,397 \\
12,009 \\
308 \\
1,080 \\
(4)\end{array}$ & $\begin{array}{r}19,741 \\
17,866 \\
276 \\
41,599 \\
(4)\end{array}$ & $\begin{array}{c}24,846 \\
23,169 \\
\left({ }^{3}\right) \\
1,396 \\
3281\end{array}$ & $\begin{array}{l}24,733 \\
22,779 \\
(3) \\
1,565 \\
390\end{array}$ & $\begin{array}{l}24,764 \\
23,012 \\
\left({ }^{3}\right) \\
1,427 \\
3324\end{array}$ & $\begin{array}{c}26,119 \\
24,356 \\
(3) \\
1,539 \\
223\end{array}$ & $\begin{array}{c}26,297 \\
24,612 \\
(3) \\
1,436 \\
3249\end{array}$ \\
\hline $\begin{array}{l}1 \text { For } 198 \mathrm{C} \text {, includes only mines that produc } \\
\text { Beginning in } 1980 \text {, includes only mines the } \\
\text { Consumption data at Coke Plants and in F } \\
\text { Consumption data at Other Industrial Plan } \\
\text { Note: Total may not equal sum of compc } \\
\text { Sources: Energy Information Administrat } \\
\text { Power Monthly 1989; and Inventory of Power } \\
\text { tion Circular 8705: Historical Fuels and Energy } \\
\text { logical Survey Bulletin 1412: Soal Resources o }\end{array}$ & $\begin{array}{l}10,000 \\
\text { produced } \\
\text { sidential } \\
\text { and in } \mathrm{R} \\
\text { ants becs } \\
\text {, Coal }\end{array}$ & $\begin{array}{l}\text { e tons. } \\
0 \text { or more } \\
\text { mmercial } \\
\text { tal and C } \\
\text { indepenc } \\
\text { otion; Que } \\
\text { d States }\end{array}$ & $\begin{array}{l}\text { r are con } \\
\text { rcial Sect } \\
\text { unding. } \\
\text { Coal Re } \\
\text { Bureau o } \\
\text { ed States } \\
74 .\end{array}$ & $\begin{array}{l}\text { to avoid } \\
\text { combine } \\
\text { Veekly C } \\
\text { s, U.S. D }\end{array}$ & $\begin{array}{l}\text { ure of ind } \\
\text { oid disclo } \\
\text { duction; } \\
\text { ent of Intr }\end{array}$ & $\begin{array}{l}\text { company } \\
\text { individua } \\
\text { ata: A } \\
\text { finerals } Y\end{array}$ & $\begin{array}{l}\text { Dany data. } \\
\text { ce; Eloctric } \\
\text { ok; Informa- } \\
\text { pi; and Goo- }\end{array}$ \\
\hline
\end{tabular}




\section{Montana}

\section{Coal Highlights}

\section{Production}

(thousand short tons)

37,742

Value

(million dollars)

Number of Employees

Consumption

(thousand short tons)

Coal-Fired Electric Generating Units

Number

Summer Capability (megawatts)

$\%$ of Total Summer Capability

Ceneration (gigawatthours)

Coal Deposits

Area (square miles)

51,300

Percent of State Land

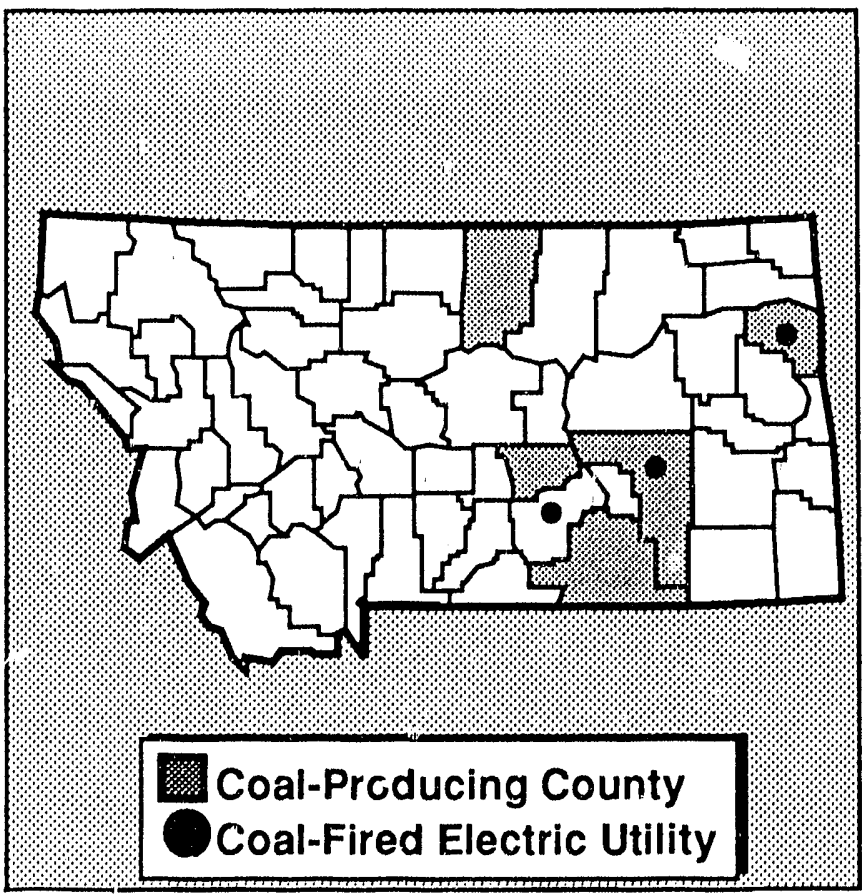

Table B20. Montana Coal Facts

\begin{tabular}{|c|c|c|c|c|c|c|c|}
\hline & 1970 & 1975 & 1880 & $1800 \pi$ & 1887 & 1888 & 1989 \\
\hline $\begin{array}{l}\text { Production (Thousand Short Tons) } \\
\text { Underground } \\
\text { Surface }\end{array}$ & $\begin{array}{r}3,447 \\
28 \\
3,419\end{array}$ & $\begin{array}{r}22,054 \\
0 \\
22,054\end{array}$ & $\begin{array}{r}29,872 \\
0 \\
29,872\end{array}$ & $\begin{array}{r}33,290 \\
0 \\
33,290\end{array}$ & $\begin{array}{r}34,399 \\
0 \\
34,399\end{array}$ & $\begin{array}{r}38,881 \\
0 \\
38,881\end{array}$ & $\begin{array}{r}37,142 \\
0 \\
37,742\end{array}$ \\
\hline Average Mine Price (Dollars per Short Ton) & 1.85 & 5.08 & 10.50 & 13.18 & 12.43 & 10.06 & 10.27 \\
\hline Total Value of Production (Million Dollars) ... & 6 & 112 & 314 & 439 & 428 & 391 & 388 \\
\hline Percentege of U.S. Coal Production .............. & .6 & 3.4 & 3.6 & 3.8 & 3.7 & 4.1 & 3.8 \\
\hline 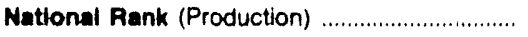 & 18 & 10 & 9 & 10 & 9 & 8 & 8 \\
\hline $\begin{array}{l}\text { Total U.S. Coal Production } \\
\text { (Thousand Short Tons) }\end{array}$ & 612,661 & 654,641 & 829,700 & 883,638 & 918,762 & 950,265 & 980,729 \\
\hline 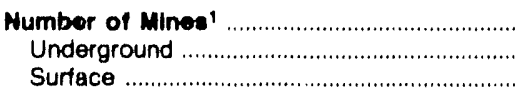 & $\begin{array}{l}8 \\
4 \\
4\end{array}$ & $\begin{array}{l}8 \\
0 \\
8\end{array}$ & $\begin{array}{l}9 \\
0 \\
9\end{array}$ & $\begin{array}{l}9 \\
0 \\
9\end{array}$ & $\begin{array}{l}8 \\
0 \\
8\end{array}$ & $\begin{array}{l}9 \\
0 \\
9\end{array}$ & $\begin{array}{l}9 \\
0 \\
\theta\end{array}$ \\
\hline 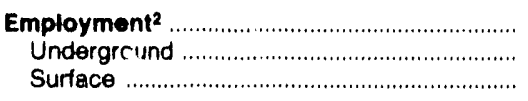 & $\begin{array}{r}109 \\
28 \\
81\end{array}$ & $\begin{array}{r}640 \\
0 \\
640\end{array}$ & $\begin{array}{r}1,131 \\
0 \\
1,131\end{array}$ & $\begin{array}{r}1,173 \\
0 \\
1,173\end{array}$ & $\begin{array}{r}847 \\
0 \\
847\end{array}$ & $\begin{array}{r}872 \\
0 \\
872\end{array}$ & $\begin{array}{r}682 \\
0 \\
682\end{array}$ \\
\hline 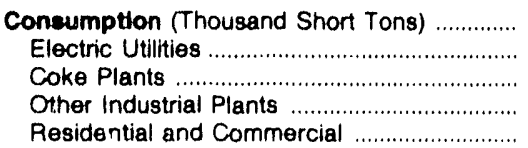 & $\begin{array}{r}1,065 \\
652 \\
0 \\
185 \\
228\end{array}$ & $\begin{array}{r}1,252 \\
1,203 \\
0 \\
49 \\
\text { (ग) }\end{array}$ & $\begin{array}{r}3,519 \\
3,351 \\
0 \\
154 \\
14\end{array}$ & $\begin{array}{r}5,713 \\
5,480 \\
0 \\
233 \\
(3)\end{array}$ & $\begin{array}{r}7,730 \\
7,530 \\
0 \\
200 \\
(3)\end{array}$ & $\begin{array}{r}10,634 \\
10,410 \\
0 \\
215 \\
\left({ }^{2}\right)\end{array}$ & $\begin{array}{r}10,468 \\
10,208 \\
0 \\
250 \\
\left({ }^{3}\right)\end{array}$ \\
\hline
\end{tabular}

1 For 1980 , includes only mines that produced 10,000 or more tons.

2 Begirining in 1980, includes only mines that produced 10,000 or more toris.

3 Consumption data at Other Industrial Plants and in Residential and Commercial Sector are combined to avoid disclosure of individual company data Note: Total may not equal sum of components because of independent rounding.

Sources. Energy Information Administration, Coal Production; Cuarterly Coal Report; Weekly Coal Production; Coal Data: A Reference; Electric Por: $x$ Monthly 1989; and Inventory of Power Plants in the United States 1989; Bureau of Mines, U.S. Department of Interior, Minerals Yeartook: Informe tion Circular 8705: H.listorical Fliels and Energy Consumption Data, 1960-72, United States by States and Census Districts West of the Mississippli and Geological Survey Bulletin 1412: Coal Resources of the United States, January 1, 1974. 


\section{Coal Highlights}

Production

(thousand short tons)

Value

(million dollars)

Number of Employees

Consumption

(thousand short tons)

Coal-Fired Electric Generating Units

Number

Summer Capability (megawatts)

\% of Total Summer Capability

Generation (gigawatthours)

Coal Deposits

Area (square miles)

Percent of State Land
23,702

12

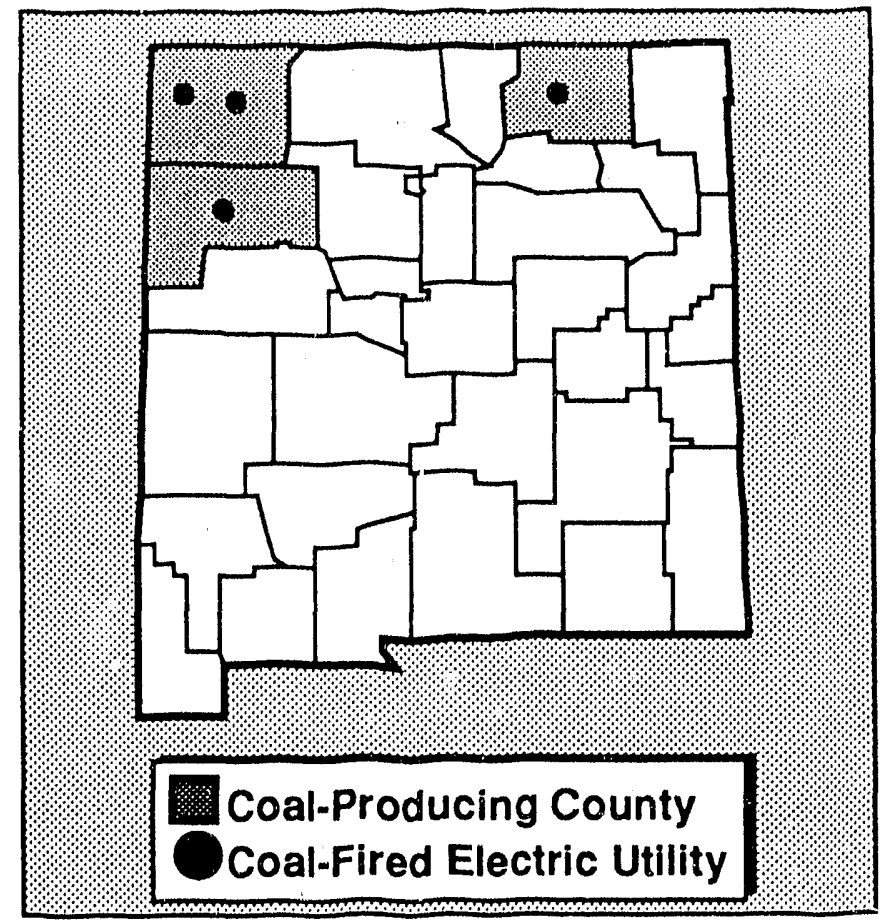

Table B21. New Mexico Coal Facts

\begin{tabular}{|c|c|c|c|c|c|c|c|}
\hline & 1870 & 1975 & 1980 & 1985 & 1987 & 1988 & 1889 \\
\hline $\begin{array}{l}\text { Production (Thousand Short Tons) } \\
\text { Underground } \\
\text { Surface }\end{array}$ & $\begin{array}{r}7,381 \\
938 \\
6,423\end{array}$ & $\begin{array}{r}8,785 \\
764 \\
8,022\end{array}$ & $\begin{array}{r}18,425 \\
950 \\
17,475\end{array}$ & $\begin{array}{r}22,203 \\
803 \\
21,398\end{array}$ & $\begin{array}{r}19,131 \\
620 \\
18,512\end{array}$ & $\begin{array}{r}21,803 \\
224 \\
21,579\end{array}$ & $\begin{array}{r}23,702 \\
45 \\
23,657\end{array}$ \\
\hline Average Mine Prlce (Dollars per Short Ton) & 2.89 & $w$ & 14.01 & 23.41 & 21.78 & 22.78 & 23.42 \\
\hline Total Value of Production (Million Dollars) ... & 21 & $w$ & 258 & $\mathbf{5 2 0}$ & 417 & 497 & 555 \\
\hline Percentage of U.S. Coal Production .............. & 1.2 & 1.3 & 2.2 & 2.5 & 2.1 & 2.3 & 2.4 \\
\hline 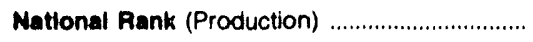 & 10 & 12 & 13 & 13 & 13 & 13 & 13 \\
\hline $\begin{array}{l}\text { Total U.S. Cosl Production } \\
\text { (Thousand Short Tons) }\end{array}$ & 612,661 & 654,641 & 820,700 & 883,638 & 918,762 & 950,266 & 980,729 \\
\hline 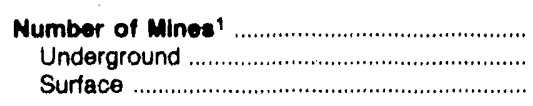 & $\begin{array}{l}4 \\
1 \\
3\end{array}$ & $\begin{array}{l}5 \\
1 \\
4\end{array}$ & $\begin{array}{r}10 \\
1 \\
9\end{array}$ & $\begin{array}{r}12 \\
2 \\
10\end{array}$ & $\begin{array}{r}10 \\
1 \\
9\end{array}$ & $\begin{array}{r}10 \\
1 \\
9\end{array}$ & $\begin{array}{l}8 \\
1 \\
7\end{array}$ \\
\hline $\begin{array}{l}\text { Employment2 } \\
\text { Underground } \\
\text { Surface }\end{array}$ & $\begin{array}{l}426 \\
165 \\
261\end{array}$ & $\begin{array}{l}930 \\
340 \\
580\end{array}$ & $\begin{array}{r}1,603 \\
390 \\
1,213\end{array}$ & $\begin{array}{r}1,929 \\
249 \\
1,680\end{array}$ & $\begin{array}{r}1,658 \\
192 \\
1,468\end{array}$ & $\begin{array}{r}1,584 \\
159 \\
1,425\end{array}$ & $\begin{array}{r}1,470 \\
49 \\
1,421\end{array}$ \\
\hline 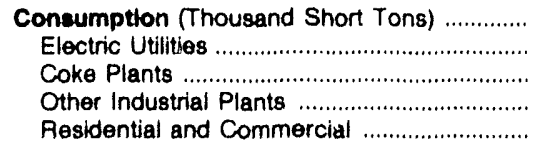 & $\begin{array}{r}6,032 \\
6,020 \\
0 \\
12 \\
0\end{array}$ & $\begin{array}{r}7,422 \\
7,422 \\
0 \\
0 \\
0\end{array}$ & $\begin{array}{r}11,468 \\
11,406 \\
0 \\
352 \\
(3)\end{array}$ & $\begin{array}{r}14,589 \\
14,498 \\
0 \\
90 \\
(3)\end{array}$ & $\begin{array}{r}14,395 \\
14,340 \\
0 \\
55 \\
(3)\end{array}$ & $\begin{array}{r}14,715 \\
14,661 \\
0 \\
351 \\
(3)^{3}\end{array}$ & $\begin{array}{r}15,295 \\
15,250 \\
0 \\
344 \\
(3)\end{array}$ \\
\hline
\end{tabular}

1 For 1980 , includes only minos that produced 10,000 or more tons.

2 Beginning in 1980 , includes only mines that produced 10,000 or more tons.

- Consumption data at Other Industrial Plants and in Pesidential and Commercial Sector are combined to avoid disclosure of individual company datal.

- Withheld to avoid disclosure of individual company data.

Note: Total may not equal sum of components because of independent rounding.

Sources: Energy Information Administralion, Coal Production; Quarterly Caal Report; Woekly Coal Production; Coal Data: A Reference; Electric Power Monthly 1989; and Inventory of Power Plants in the United States 1989; Bureau of Mines, U.S. Departrnent of Interior, Minerals Yearbook; Intorima. tion Circular 8705: Historical Fuels and Energy Consumption Data, 1960-72, United States by States and Census Districts West of the Mississippi; and Glealogical Survey Bulletin 1412: Coal Resources of the United States, January 1, 1974. 


\section{North Dakota}

\section{Coal Highlights}

Production

(thousand short tons)

Value

(million dollars)

Number of Employees

Consumption

(thousand short tons)

Coal-Fired Electric Generating Units

Number

Summer Capability (megawatts)

$\%$ of Total Summer Capability

Generation (gigawatthours)

Coal Deposits

Area (square miles)

Percent of State Land
29,566

218

850

27,401

14

3,876

85.7

23,774

32,000

45

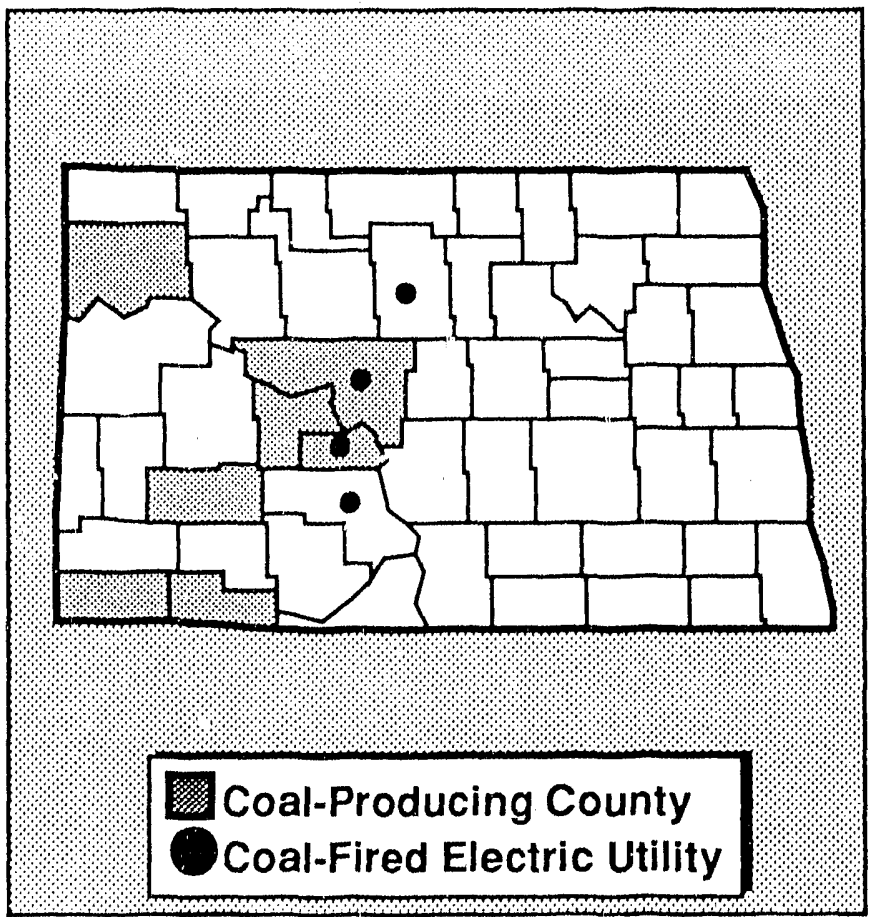

Table B22. North Dakota Coal Facts

\begin{tabular}{|c|c|c|c|c|c|c|c|}
\hline & 1970 & 1975 & 1980 & 1985 & 1987 & 1988 & 1989 \\
\hline $\begin{array}{l}\text { Production (Thousand Short Tons) } \\
\text { Underground } \\
\text { Surface }\end{array}$ & $\begin{array}{r}5,639 \\
0 \\
5,639\end{array}$ & $\begin{array}{r}8,515 \\
0 \\
8,515\end{array}$ & $\begin{array}{r}16,975 \\
0 \\
16,975\end{array}$ & $\begin{array}{r}26,873 \\
0 \\
26,873\end{array}$ & $\begin{array}{r}25,142 \\
0 \\
25,142\end{array}$ & $\begin{array}{r}29,731 \\
0 \\
29,731\end{array}$ & $\begin{array}{r}29,566 \\
0 \\
29,566\end{array}$ \\
\hline Average Mine Price (Dollars per Short Ton) & 1.95 & 3.17 & 7.48 & 9.30 & 7.91 & 7.38 & 7.36 \\
\hline Total Value of Production (Million Dollars) ... & 11 & 27 & 127 & 250 & 199 & 210 & 218 \\
\hline Percentage of U.S. Coal Production .............. & .9 & 1.3 & 2.0 & 3.0 & 2.7 & 3.1 & 3.0 \\
\hline 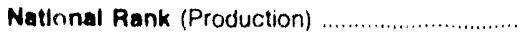 & 13 & 13 & 14 & 12 & 12 & 11 & 11 \\
\hline $\begin{array}{l}\text { Total U.S. Coal Production } \\
\text { (Thousand Short Tons) }\end{array}$ & 612,661 & 654,641 & $\mathbf{8 2 9}, 700$ & 983,638 & 918,762 & 950,265 & 980,729 \\
\hline $\begin{array}{l}\text { Number of Mines } 1 \\
\text { Underground } \\
\text { Surface }\end{array}$ & $\begin{array}{r}20 \\
0 \\
20\end{array}$ & $\begin{array}{r}10 \\
0 \\
10\end{array}$ & $\begin{array}{r}13 \\
0 \\
13\end{array}$ & $\begin{array}{r}14 \\
0 \\
14\end{array}$ & $\begin{array}{r}11 \\
0 \\
11\end{array}$ & $\begin{array}{r}12 \\
0 \\
12\end{array}$ & $\begin{array}{r}13 \\
0 \\
13\end{array}$ \\
\hline $\begin{array}{l}\text { Employment } \\
\text { Underground } \\
\text { Surtace }\end{array}$ & $\begin{array}{r}306 \\
0 \\
306\end{array}$ & $\begin{array}{r}380 \\
0 \\
380\end{array}$ & $\begin{array}{r}1,711 \\
0 \\
1,711\end{array}$ & $\begin{array}{r}1,132 \\
0 \\
1,132\end{array}$ & $\begin{array}{r}961 \\
0 \\
961\end{array}$ & $\begin{array}{r}977 \\
0 \\
977\end{array}$ & $\begin{array}{r}850 \\
0 \\
850\end{array}$ \\
\hline $\begin{array}{l}\text { Consumption (Thousand Short Tons) } \\
\text { Electric Utilities } \\
\text { Coke Plants } \\
\text { Other Industrial Plants } \\
\text { Residential and Cornmercial }\end{array}$ & $\begin{array}{r}4,799 \\
4,103 \\
0 \\
489 \\
207\end{array}$ & $\begin{array}{r}5,650 \\
5,068 \\
0 \\
3582 \\
(3)\end{array}$ & $\begin{array}{r}12,347 \\
11,619 \\
0 \\
3728 \\
(3)\end{array}$ & $\begin{array}{r}22,958 \\
17,354 \\
0 \\
3,604 \\
(3)\end{array}$ & $\begin{array}{r}24,101 \\
17,434 \\
0 \\
6,667 \\
(3)\end{array}$ & $\begin{array}{r}28,028 \\
21,686 \\
0 \\
36,342 \\
(3)\end{array}$ & $\begin{array}{r}27,401 \\
20,536 \\
0 \\
36,863 \\
(3)\end{array}$ \\
\hline
\end{tabular}

1 For 1980, includes only mines that produced 10,000 or more tons

2 Beginning in 1980, includes only mines that produced 10,000 or more tons.

3 Consumption data at Other Industrial Plants and in Residential and Commercial Sector are combined to avord disclosure of individual company data. Note: Total may not equal sum of components because of independent rounding.

Sources: Energy Information Administration, Coal Production; Quarterly Coal Report; Weokly Coal Production: Coal Data: A Raferenco: Eloctric Power Monthly 1989; and Inventory of Power Plants in the United States 1989; Bureau of Mines, U.S. Departrnent of Interior, Minerals Yearbook; Intorma tion Circular 8705: Historical Fuels and Energy Consumption Data, 1960.72. United States by States and Census Districts West of the Mississippi; and Geo. logical Survey Bulletin 1412: Coal Resources of the United States, January 1, 1974. 


\section{Coal Highlights}

Production

(thousand short tons)

Value

(raillion dollars)

Number of Employees

Consumption

(thousand short tons)

Coal-Fired Electric Generating Units

Number

Summer Capability (megawatis)

\% of Total Summer Capability

Generation (gigawatthours)

\section{Coal Deposits}

Area (square miles)

Percent of State Larid

\section{1,027}

7,374

61,016

117,677

i0,000

24

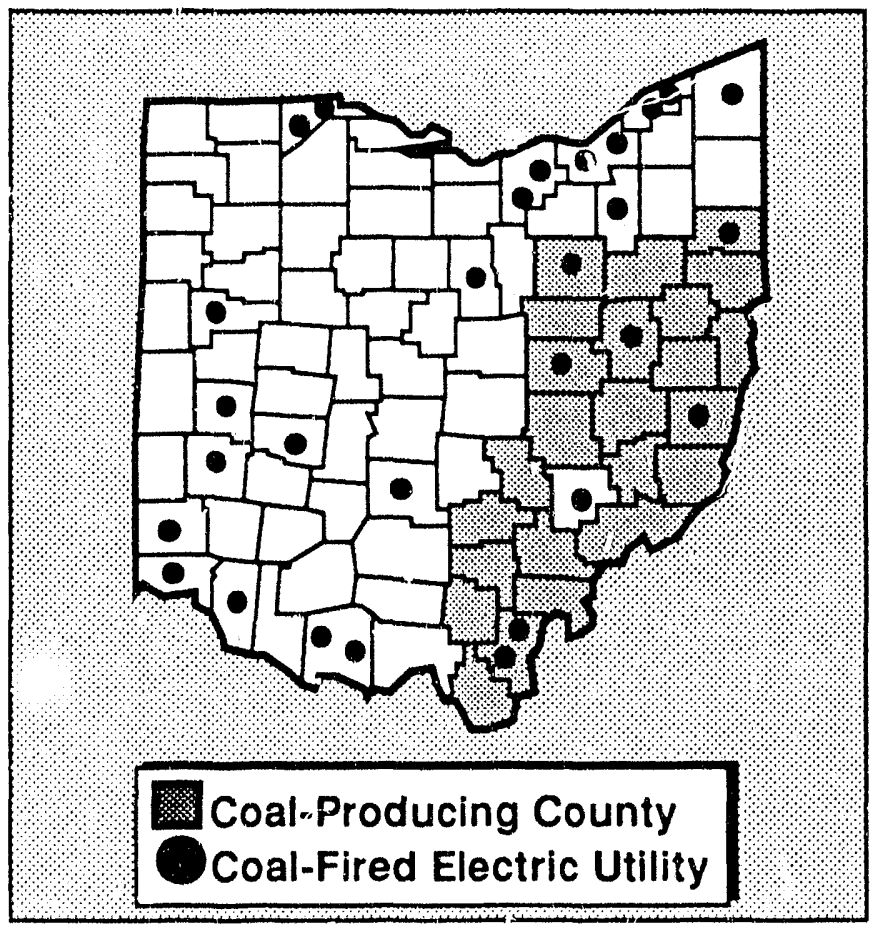

Table B23. Ohio Coal Facts

\begin{tabular}{|c|c|c|c|c|c|c|c|}
\hline & 1970 & 1975 & 1980 & 1985 & 1987 & 1988 & 1989 \\
\hline $\begin{array}{l}\text { Production (Thousand Short Tons) } \\
\text { Underground } \\
\text { Surtace }\end{array}$ & $\begin{array}{l}55,351 \\
18,111 \\
37,240\end{array}$ & $\begin{array}{l}46,770 \\
15,455 \\
31,315\end{array}$ & $\begin{array}{l}39,394 \\
12,994 \\
26,400\end{array}$ & $\begin{array}{l}35,602 \\
13,646 \\
21,956\end{array}$ & $\begin{array}{l}35,788 \\
12,317 \\
23,171\end{array}$ & $\begin{array}{l}34,043 \\
11,264 \\
22,779\end{array}$ & $\begin{array}{l}33,689 \\
10,824 \\
22,865\end{array}$ \\
\hline Averege Mine Price (Dollars per Short Ton). & 4.74 & $16.4 n$ & 27.82 & 33.87 & 30,80 & 30.50 & 30.48 \\
\hline Total Valwe of Production (Million Dollars) ... & 262 & 767 & 1,098 & 1,200 & 1,102 & 1,038 & 1,027 \\
\hline Percentege of U.S. Cosl Production ............. & 9.0 & 7.1 & 4.7 & 4.0 & 3.9 & 3.6 & 3.4 \\
\hline 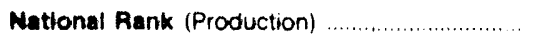 & 5 & 5 & 7 & 8 & 8 & 9 & 8 \\
\hline $\begin{array}{l}\text { Total U.S. Coal Production } \\
\text { (Thousand Short Tons) .... }\end{array}$ & 612,661 & 654,641 & 8329,700 & 883,638 & 918,672 & 950,265 & 980,729 \\
\hline $\begin{array}{l}\text { Number of Mines' } \\
\text { Underground } \\
\text { Surtace }\end{array}$ & $\begin{array}{r}306 \\
44 \\
262\end{array}$ & $\begin{array}{r}348 \\
33 \\
315\end{array}$ & $\begin{array}{r}199 \\
19 \\
180\end{array}$ & $\begin{array}{r}204 \\
14 \\
190\end{array}$ & $\begin{array}{r}210 \\
16 \\
194\end{array}$ & $\begin{array}{r}215 \\
16 \\
199\end{array}$ & $\begin{array}{r}184 \\
14 \\
170\end{array}$ \\
\hline $\begin{array}{l}\text { Employment } \\
\text { Underground } \\
\text { Surtace }\end{array}$ & $\begin{array}{l}9,251 \\
5,044 \\
4,207\end{array}$ & $\begin{array}{r}13,430 \\
8,730 \\
4,700\end{array}$ & $\begin{array}{r}14,638 \\
7,843 \\
6,795\end{array}$ & $\begin{array}{l}8,954 \\
4,166 \\
4,788\end{array}$ & $\begin{array}{l}7,827 \\
3,552 \\
4,275\end{array}$ & $\begin{array}{l}7,106 \\
2,860 \\
4,246\end{array}$ & $\begin{array}{l}7,374 \\
2,953 \\
4,421\end{array}$ \\
\hline $\begin{array}{l}\text { Consumption (Thousand Short Tons) } \\
\text { Electric Utilities } \\
\text { Coke Plants } \\
\text { Other industrial Plants } \\
\text { Residential and Commercial }\end{array}$ & $\begin{array}{r}67,375 \\
37,124 \\
12,734 \\
17,517 \\
(3)\end{array}$ & $\begin{array}{l}68,019 \\
46,389 \\
12,515 \\
39,115 \\
(3)\end{array}$ & $\begin{array}{r}64,914 \\
48,537 \\
10,268 \\
5.553 \\
556\end{array}$ & $\begin{array}{r}57,979 \\
46,700 \\
5,242 \\
5,178 \\
859\end{array}$ & $\begin{array}{r}59,350 \\
47,520 \\
5,361 \\
5,657 \\
812\end{array}$ & $\begin{array}{r}61,096 \\
48,993 \\
5,448 \\
6,030 \\
726\end{array}$ & $\begin{array}{r}61,016 \\
50,479 \\
5,265 \\
4,727 \\
545\end{array}$ \\
\hline
\end{tabular}

1. For 1980 , inctudes only mines that produced 10,000 or more tons.

2 Beginning in 1980, includes only mines that produced 10,000 or more tons.

- Consumption data at Other Industrial Plants and in Residential and Commercial Sector are combined to avoid disclosure of individual company data

Note: Total may not equal sum of components because of independent rounding.
Sources: Energy Information Administration, Coal Production; Ouarterty Coal Reoort; Wrekly Coar Production; Coal Data: A Relerence; Electrk Power Monthiy 1989; and triventory of Power Plents in the United Stales 1989; Bureau of Mines, US. Departnient of interior, Minerals Yearbisok; Intorme.

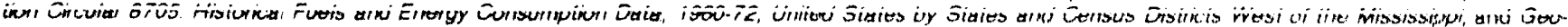
logical Survey Bulletin 1412. Coal Resources of the Uniled States, January 1. 1974. 


\section{Oklahoma}

\section{Coal Highlights}

Production

(thousand short tons)

Value

(million dollars)

Number of Employees

Consumption

(thousand short tons)

Coal-Fired Electric Generating Units

Number

Summer Capability (megawatts)

$\%$ of Total Surnmer Capability

Generation (gigawatthours)

\section{Coal Deposits}

Area (square miles)

Percent of State Land
1,753

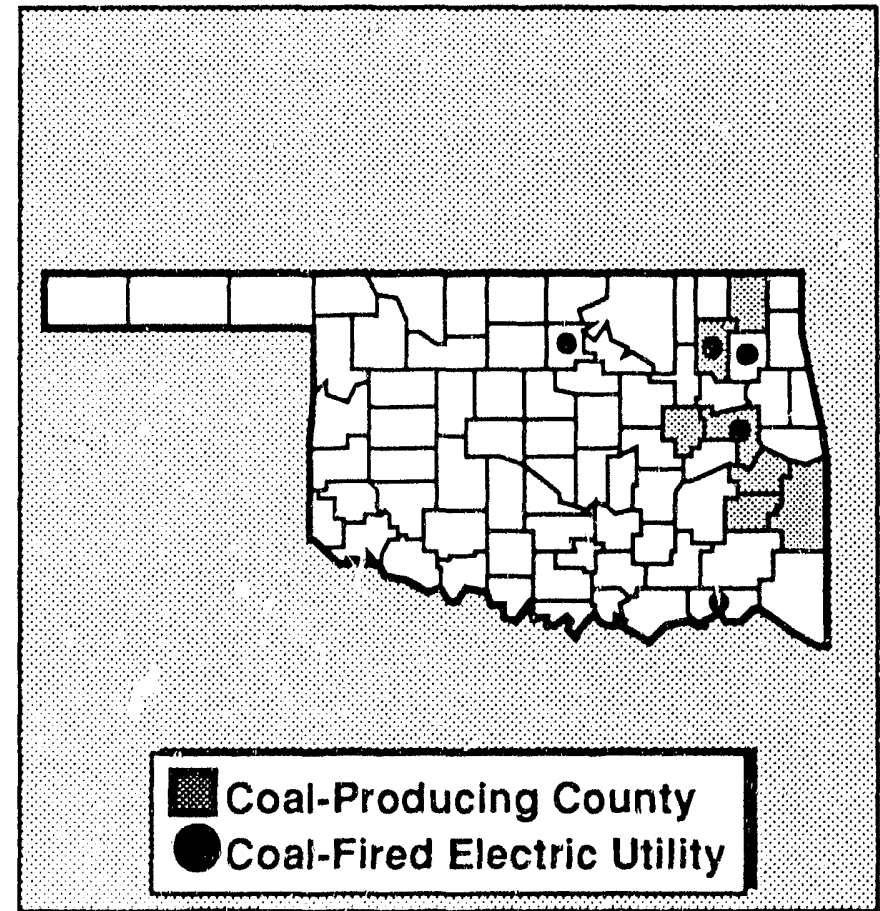

Table B24. Oklahoma Coal Facts

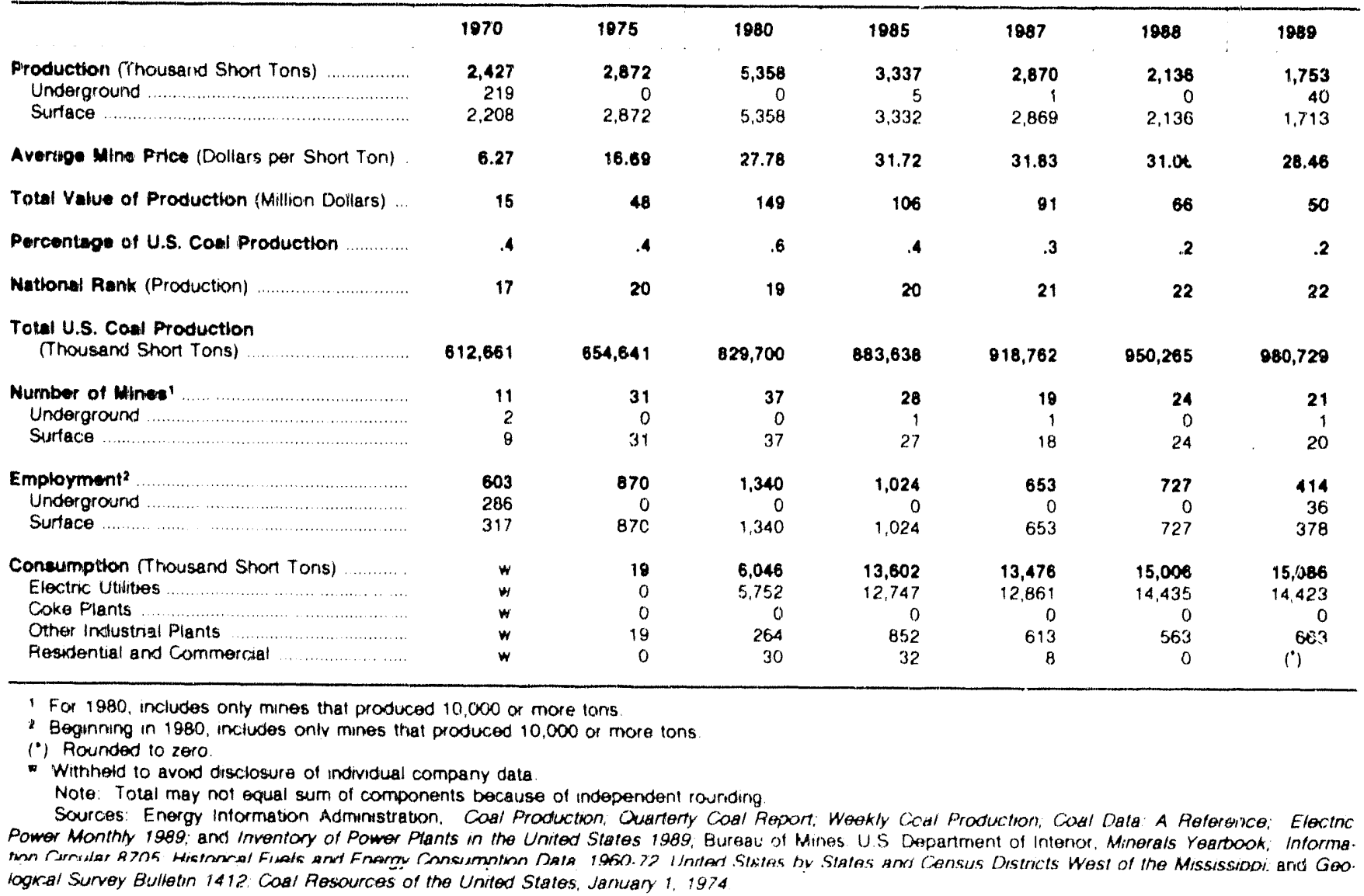




\section{Coal Highlights}

Production

(thousand short tons)

70,596

Value

(million dollars)

Number of Employees

15,469

Consumption

(thousand short tons)

58,526

Coal-Fired Electric Generating Units

Number

Summer Capability (megawatts)

\% of Total Summer Capability

Generation (gigawatthours)

Coal Deposits

Area (square miles)

Percent of State Land

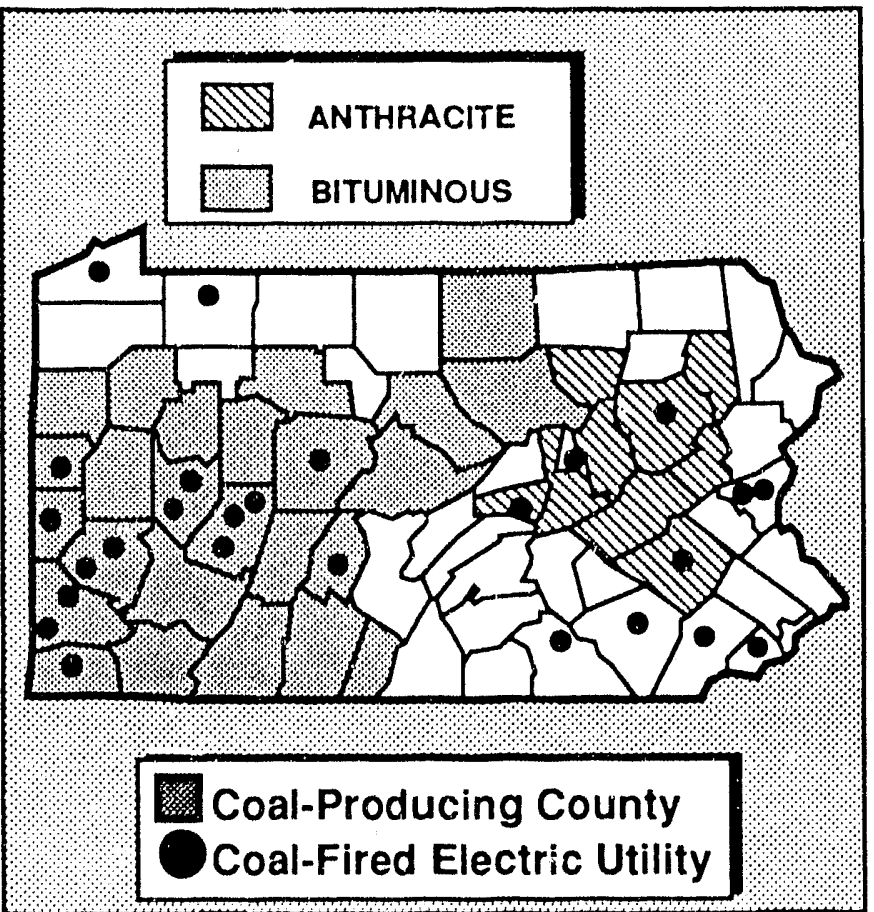

Table B25. Pennsylvania Coal Facts

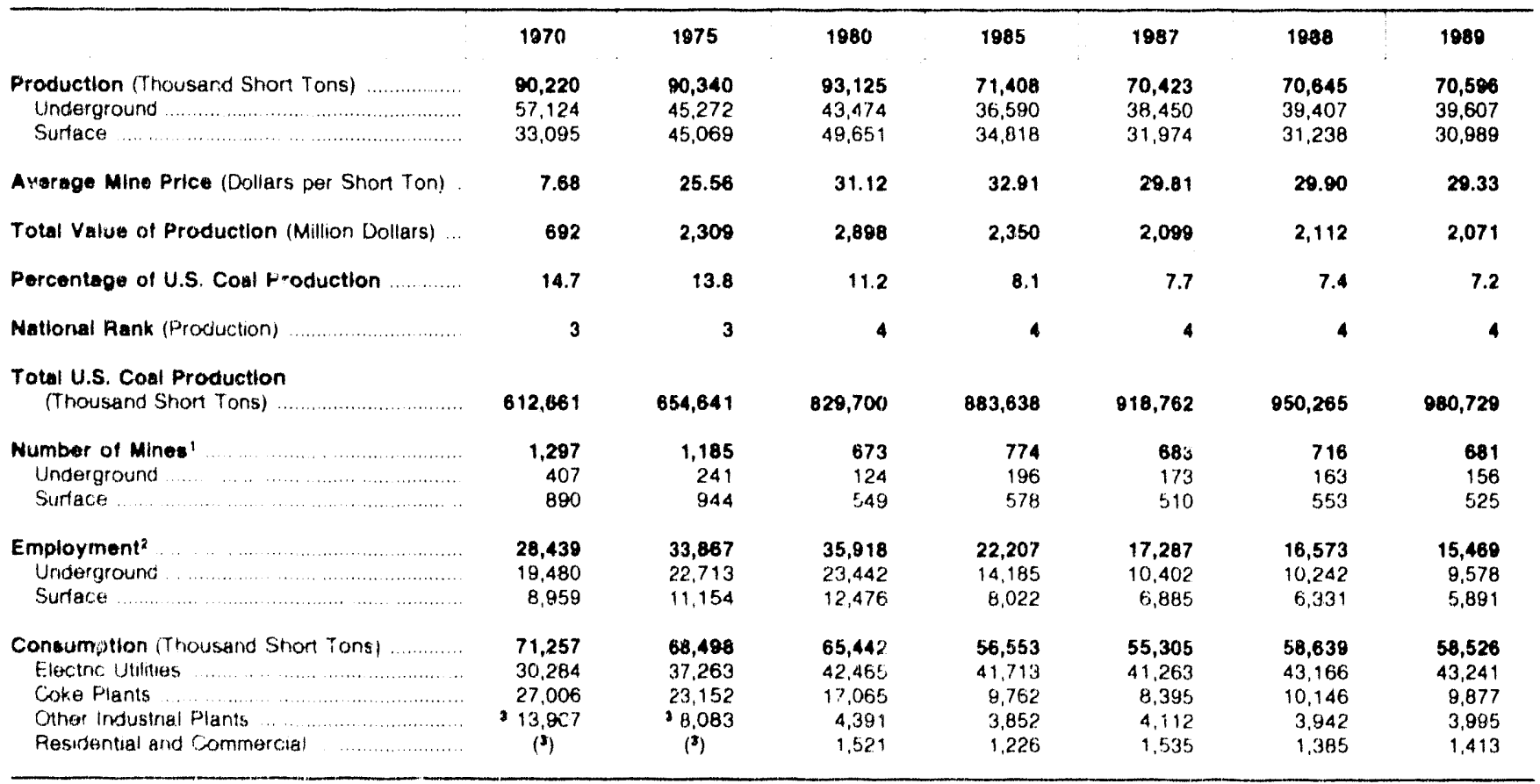

1 For 1980, includes only mines that produced 10,000 or more tons

2 Begirinirg in 1980, includes only mines tha! prodikced 10,000 or more tons.

3 Conisumption date at Other industnal Plants aro in Residential and Commercial Sector are combined to averd discicsure of individual company data. Note Total may not equal sum of components because of independent rounding.

Sources. Eriergy Information Administration, Coal Production, Quarterty Coal Report: Workt, Sual Productiori. Coal Lata. A Roference: Eloctric Power Monthly 1989; and Inventory of Power Plants in the United States 1989; Bureau of Miries. U.S. Department of interior. Minerals Yearbook: Informa. tron Circular 8705 . Histoncal Fuels and Energy Consumption Data, 1960-72. United States by Stales and Cansus Districts West of the Mississippi and Geo

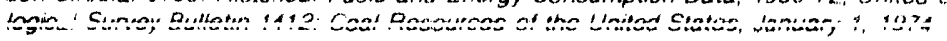




\section{Tennessee}

\section{Coal Highlights}

Production

(thousand short tons)

6,480

Value

(million dollars)

Number of Employees

Consumption

(thousand short tons)

Coal-Fired Electric Generating Units

Number

Summer Capability (megawatts)

9,289

$\%$ of Total Summer Capability

Generation (gigawatthours)

46,324

\section{Coal Deposits}

Area (square miles)

Percent of State Land

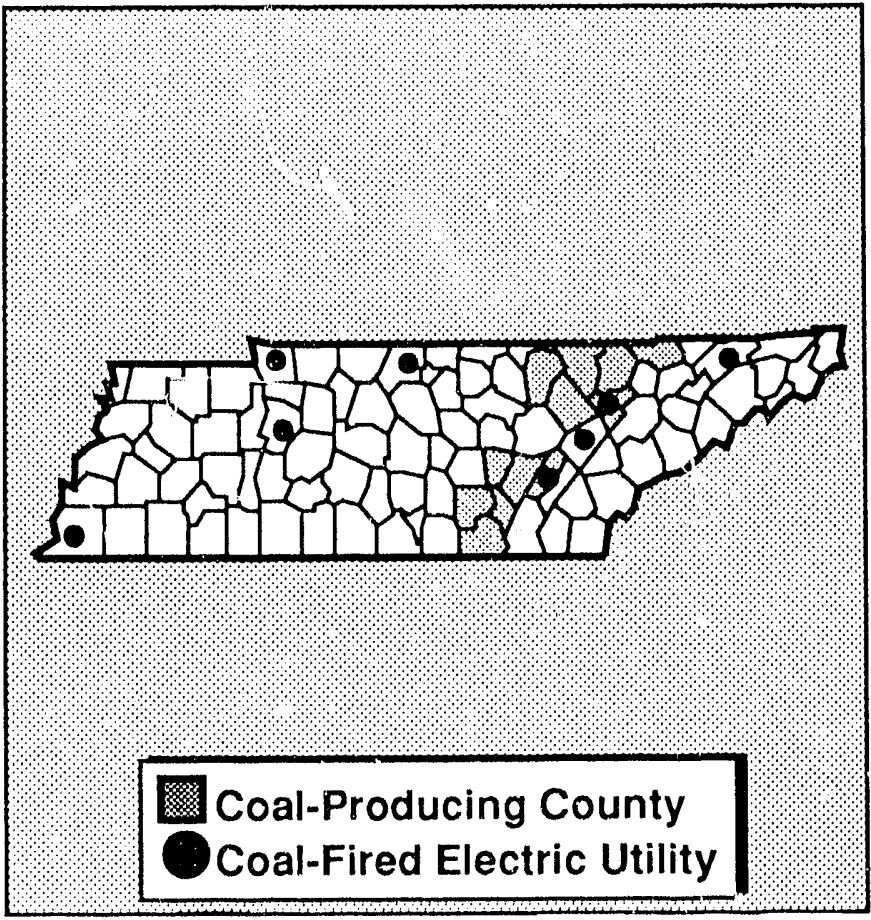

Table B26. Tennessee Coal Facts

\begin{tabular}{|c|c|c|c|c|c|c|c|}
\hline 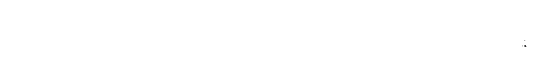 & 1870 & 1975 & 1980 & 1985 & 1987 & 1988 & 1989 \\
\hline Production (Thousand Short Tons) ................ & 8,237 & 8,206 & 9,850 & 7,446 & 6,442 & 6,510 & 6,480 \\
\hline 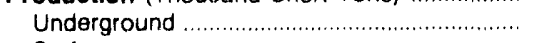 & 4,350 & 3,806 & 4,750 & 5,204 & 4,865 & 4,689 & 4,688 \\
\hline Surface & 3,886 & 4,400 & 5.100 & 2,242 & 1,577 & 1,821 & 1,792 \\
\hline Average Mine Price (Dollars per Short Ton) & 4.90 & 17.10 & 27.54 & 28.54 & 27.65 & 26.78 & 26.98 \\
\hline Total Value of Production (Million Dollars) ... & 40 & 140 & 271 & 213 & 178 & 190 & 175 \\
\hline Percentage of U.S. Coal Production ............. & 1.3 & 1.3 & 1.2 & .8 & .7 & .7 & .7 \\
\hline 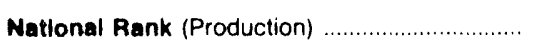 & 9 & 15 & 17 & 17 & 17 & 17 & 17 \\
\hline $\begin{array}{l}\text { Total U.S. Coal Production } \\
\quad \text { (Thousand Short Tons) }\end{array}$ & 612,661 & 654,641 & 829,700 & 883,638 & 918,762 & 950,265 & 980,729 \\
\hline Number of Mines' & 203 & 166 & 117 & 118 & 83 & 105 & 98 \\
\hline 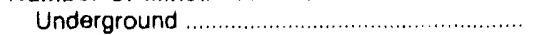 & 116 & 62 & 54 & 70 & 60 & 74 & 68 \\
\hline Surface & 87 & 104 & 63 & 48 & 33 & 31 & 30 \\
\hline 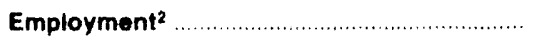 & 1,999 & 3,140 & 3,615 & 2,622 & 1,998 & 2,119 & 1,857 \\
\hline Underground & 1,344 & 1,890 & 2,076 & 1.871 & 1,552 & 1,676 & 1,471 \\
\hline 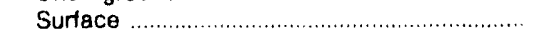 & 655 & 1,250 & 1,539 & 751 & 446 & 443 & 386 \\
\hline Consumption (Thousand Short Tons) ............. & 18,315 & 26,633 & 24,496 & 25,105 & 24,782 & 25,152 & 23,496 \\
\hline Electric Utilities & 15,494 & 24,662 & 21,679 & 20,853 & 20,697 & 21,010 & 19,283 \\
\hline 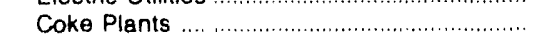 & 183 & 160 & $\left({ }^{3}\right)$ & (3) & $\left({ }^{3}\right)$ & $\left({ }^{3}\right)$ & $\left({ }^{3}\right)$ \\
\hline Other Industrial Plants & $(4)$ & $(4)$ & $\left(3^{\prime}\right)$ & $\left({ }^{3}\right)$ & $(3$ & $(3)$ & ${ }^{(3)}$ \\
\hline 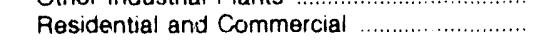 & 42,638 & 41,811 & 32,817 & 34,253 & 34,186 & 34.141 & 4,213 \\
\hline
\end{tabular}

1 For 1980 , includes only mines that produced 10,000 or more tons.

2 Beginning in 1980 . includes only mines that produced 10,000 or more tons.

3 Consumption data at Coke Plants, Other Industrial Plants and in Residential and Commerrial Sector are combiried to avoid disclosure of individual company data.

4 Consumption data at Other Industrial Plants and in Residential and Commercial Sector are combined to avoid disclosure of individual company data. Note: Total may not equal sum of components because of independent rounding.

Sources: Energy Intormation Administration, Coal Production; Quarterly Coal Report: Woek', Coai Production; Coal Data: A Reterence; Electric Power Monthly 1989; and Inventory of Power Plants in the United States 1989; Bureau of Mines. U.S. Department of interior, Minerals Yeartiook; Intorma. tion Circular 8705: Histionical Fueis and Energy Consumption Data, 1960-72. United States by States and Census Districts West of the Mississippi; and Geolooical Survev Bulletın 1412: Coal Resources of the United States, January 1, 1974. 


\section{Coal Highlights}

Production

(thousand short tons)

53,854

Value

(million dollars)

Number of Employees

Consumption

(thousand short tons)

90,989

Coal-Fired Electric Grating Units

Number

Summer Capability (megawatts)

$\%$ of Total Summer Capability

Generation (gigawathours)

\section{Coal Deposits}

Area (square miles)

Percent of State Land

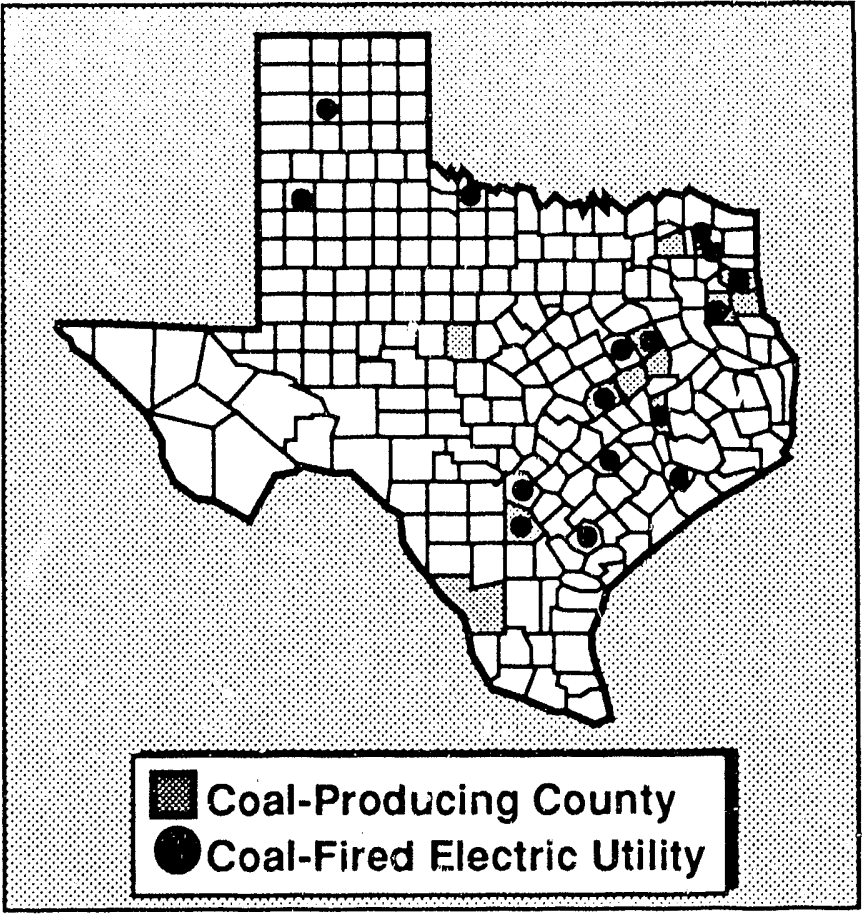

Table B27. Texas Coal Facts

\begin{tabular}{|c|c|c|c|c|c|c|c|}
\hline & 1970 & 1975 & 1980 & 1985 & $198 i$ & 1988 & 1989 \\
\hline Production (Thousand Short Tons) .................. & 0 & 11,002 & 29,354 & 45,459 & 50,529 & 52,281 & 53,854 \\
\hline 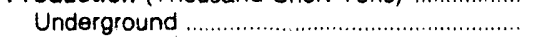 & 0 & & & & & 0 & \\
\hline Surtace & 0 & 11,002 & 29,354 & 45,459 & 50,529 & 52,281 & 53,854 \\
\hline Average Mine Price (Dollars per Short Ton) & .00 & $w$ & 7.67 & 11.60 & 12.10 & 11.34 & 10.91 \\
\hline Total Value of Production (Million Dollars) ... & 0 & w & 225 & 527 & 611 & 593 & 588 \\
\hline Percentage of U.S. Coal Production .............. & .0 & 1.7 & 3.5 & 5.1 & 5.5 & 5.5 & 5.5 \\
\hline National Rarik (Production) ............................... & 0 & 11 & 10 & 6 & 6 & 6 & 6 \\
\hline $\begin{array}{l}\text { Total U.S. Coal Production } \\
\text { (Thousand Short Tons) }\end{array}$ & 612,661 & 654,641 & 828,700 & 883,638 & 818,762 & 950,265 & 980,728 \\
\hline Number of Mines' ${ }^{\prime}$ & 0 & 4 & 10 & 14 & 15 & 14 & 15 \\
\hline 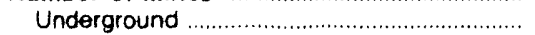 & 0 & 0 & 0 & 0 & 0 & 0 & 0 \\
\hline 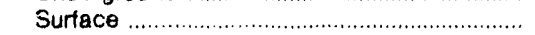 & $\mathrm{c}$ & 4 & 10 & 14 & 15 & 14 & 15 \\
\hline Emplorment ${ }^{2}$. & 0 & 440 & 1,780 & 2,360 & 3,319 & 3,057 & 2,109 \\
\hline 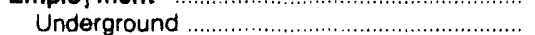 & 0 & 0 & 0 & 0 & 0 & 0 & 0 \\
\hline Surface & 0 & 440 & 1,780 & 2,360 & 3,319 & 3,057 & 2,108 \\
\hline Coneumption (Thousand Short Tons) .............. & w & 12,370 & 48,602 & 77,017 & 82,915 & 86,644 & 90,988 \\
\hline Electric Utilities ...................................... & $w$ & 9,067 & 45,351 & 71,818 & 78,802 & 82,889 & 86,701 \\
\hline 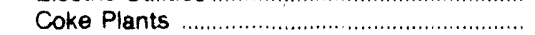 & $\mathbf{w}$ & 977 & $\left(\mathbf{3}^{3}\right)$ & $\left(\mathbf{3}^{3}\right)$ & $\left({ }^{3}\right)$ & 0 & \\
\hline Other Industrial Plants ..................................... & it & 42,326 & 3,251 & 3 5,199 & 34,113 & 43,755 & 44,288 \\
\hline Residential and Commercial ............................ & $\mathbf{w}$ & (4) & $(3)$ & $\left(3^{3}\right)$ & ${ }^{(3)}$ & (4) & (4) \\
\hline
\end{tabular}

1 For 1980 includes only mines that produced 10,000 or more tons

2 Beginning in 1980, includes only mines that produced 10,000 or more tons.

- Consumption data at Coke Plants, Other Industrial Plants and in Residential and Commercial Sector are combined to avoid disclosure of individual company date.

- Consumption data at Other Industrial Plants and in Residential and Commercial Sector are combined to avoid disclosure of individual company data

- Withineld to avoid disclosure of individual company data.

Note: Total may not equal sum of components because of independent rounding.

Sources: Energy Information Administration, Coal Production; Quarterty Coal Report; Weekly Coal Production; Coal Data: A Reforence; Electric Power Monthly 1989; and Inventory of Power Plants in the United States 1989; Bureau of Mines, U.S. Department of Interior, Minerals Yegrbook; Informa. tion Circular 8705: Historical Fuels and Energy Consumption Deta, 1960.72, United States by States and Census Districts West of the Mississippi: and GeO logical Survey Bulletin 1412: Cosl Resources of the United States, January 1, 1974. 


\section{Coal Highlights}

Production

(thousand short tons)

20,102

Value

(million dollars)

Number of Employees

2,169

Consumption

(thousand short tons)

15,044

Coal-Fired Electric Generating Units

Number

Summer Capability (megawatts)

$\%$ of Total Summer Capability

Generation (gigawatthours)

29,676

Coal Deposits

Area (square miles)

15,000

Percent of State Land

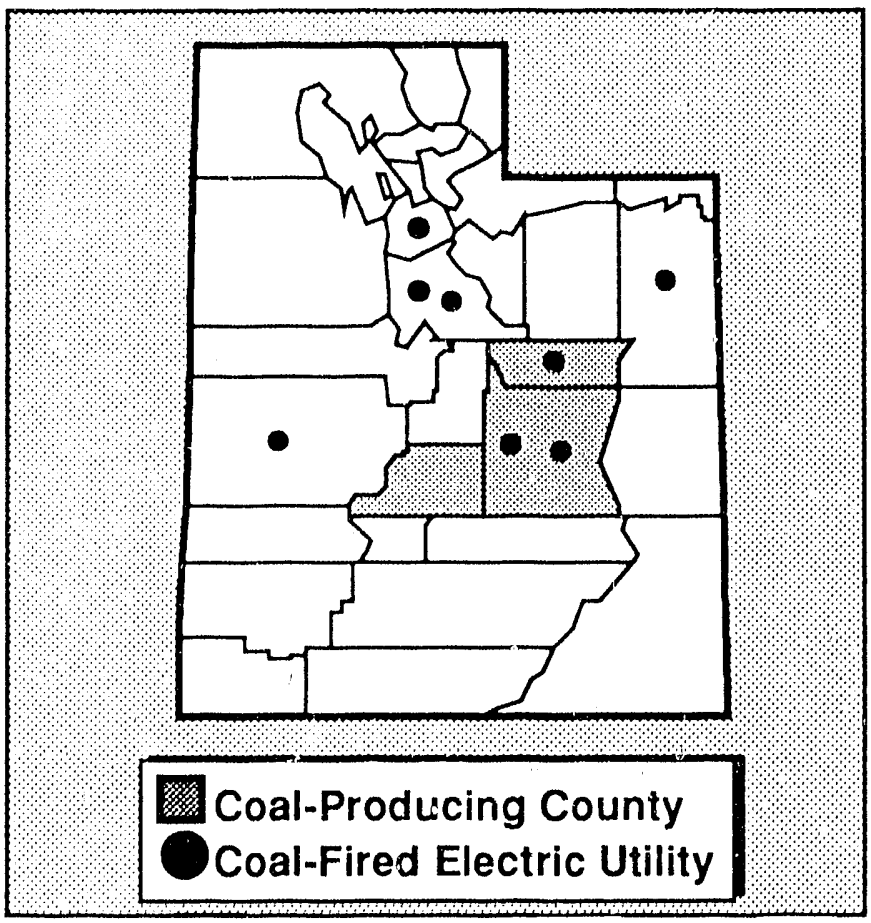

Table B28. Utah Coal Facts

\begin{tabular}{|c|c|c|c|c|c|c|c|}
\hline & 1970 & 1975 & 1980 & 1985 & 1987 & 1988 & 1989 \\
\hline Production (Thousand Short Tons) ..................... & 4,733 & 6,961 & 13,236 & 12,780 & 16,508 & 18,163 & 20,102 \\
\hline Underground & 4,733 & 6,961 & 13,236 & 12,780 & 16,508 & 18,163 & 20,102 \\
\hline Surface & 0 & 0 & 0 & 0 & 0 & 0 & 0 \\
\hline Average Mine Price (Dollars per Short Ton) & 7.28 & 19.84 & 25.63 & 27.69 & 25.70 & 22.42 & 21.46 \\
\hline Total Value of Production (Million Dollars) ... & 34 & 138 & 339 & 354 & 424 & 407 & 431 \\
\hline Percentage of U.S. Coal Production ............... & .8 & 1.1 & 1.6 & 1.4 & 1.8 & 1.8 & 2.0 \\
\hline 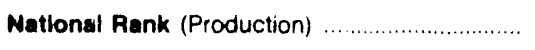 & 14 & 17 & 15 & 15 & 14 & 14 & 14 \\
\hline $\begin{array}{l}\text { Total U.S. Coal Production } \\
\text { (Thousand Short Tons) }\end{array}$ & 612,661 & 654,641 & 829,700 & 883,638 & 9/8,762 & 950,265 & 980,729 \\
\hline Number of Mines' & 20 & 20 & 29 & 22 & 21 & 20 & 21 \\
\hline 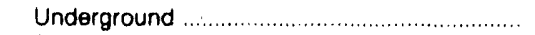 & 20 & 20 & 29 & 22 & 21 & 20 & 21 \\
\hline Surfaco & 0 & 0 & 0 & 0 & 0 & 0 & 0 \\
\hline Employment ${ }^{2}$ & 1,469 & 2,550 & 3,512 & 2,563 & 2,544 & 2,375 & 2,169 \\
\hline 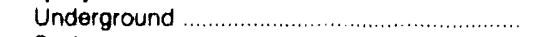 & 1.469 & 2,550 & 3,512 & 2,563 & 2,544 & 2,375 & $2,16 \theta$ \\
\hline Surface & 0 & 0 & 0 & 0 & 0 & 0 & 0 \\
\hline Consumption (Thousand Short Tons) ................ & 3,010 & 4,514 & 7,106 & 8,303 & 11,807 & 14,513 & 15,014 \\
\hline Electric Utilities & 464 & 1,995 & 4,895 & 6,325 & 11,175 & 12,544 & 12,949 \\
\hline 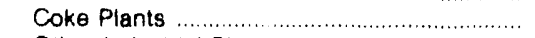 & 1,938 & 1,973 & 31,710 & 31,506 & 30 & 1,586 & 31,408 \\
\hline Other Industrial Plants & 4608 & 4546 & 501 & 472 & 631 & 4597 & 586 \\
\hline Residential and Commercial ......................... & $(4)$ & $(4)$ & $(3)$ & $(3)$ & $\left({ }^{3}\right)$ & (3) & $(3)$ \\
\hline
\end{tabular}

1 For 1980 , includes only mines that produced 10,000 or more tons

2 Beyinning in 1980, includes only mines that produced 10,000 or more tons.

3 Consumption data at Coke Plants and in Residential and Commercial Sector are combined to avord disclosure of individual company dat

- Consumption data at Other Industrial Plants and in Residential and Commercial Sector are combined to avoid disciosure of individual company data. Note: Total may not equal sum of components because of independent rounding.

Sources: Energy Intormation A.dministration, Coal Production; Quarterly Coal Report; Weskly Coal Production: Coal Dala: A Reterence; Electric Power Monthly 1989; and Inventory of Power Plants in the United States 1989; Bureau of Mines. U.S. Department of Iriterior. Minerals Yearticok; Informa. tion Circular 8705: Histonical Fuels and Energy Consumption Data, 1960-72, United Stales by States and Census Districts West uf the Mississippi; and GeO logical Survey Bulletin 1412. Coal Resources of the United States, January 1, 1.974. 


\section{Coal Highlights}

Production

(thousand short tons)

43,006

Value

(million dollars)

Number of Employees

10,371

Consumption

(thousand short tons)

Coal-Fired Electric Generating Units

Number

Summer Capability (megawatts)

\% of Toral Summer Capability

Generation (gigawatthours)

24,059

\section{Coal Deposits}

Area (square miles)

Percent of State Land

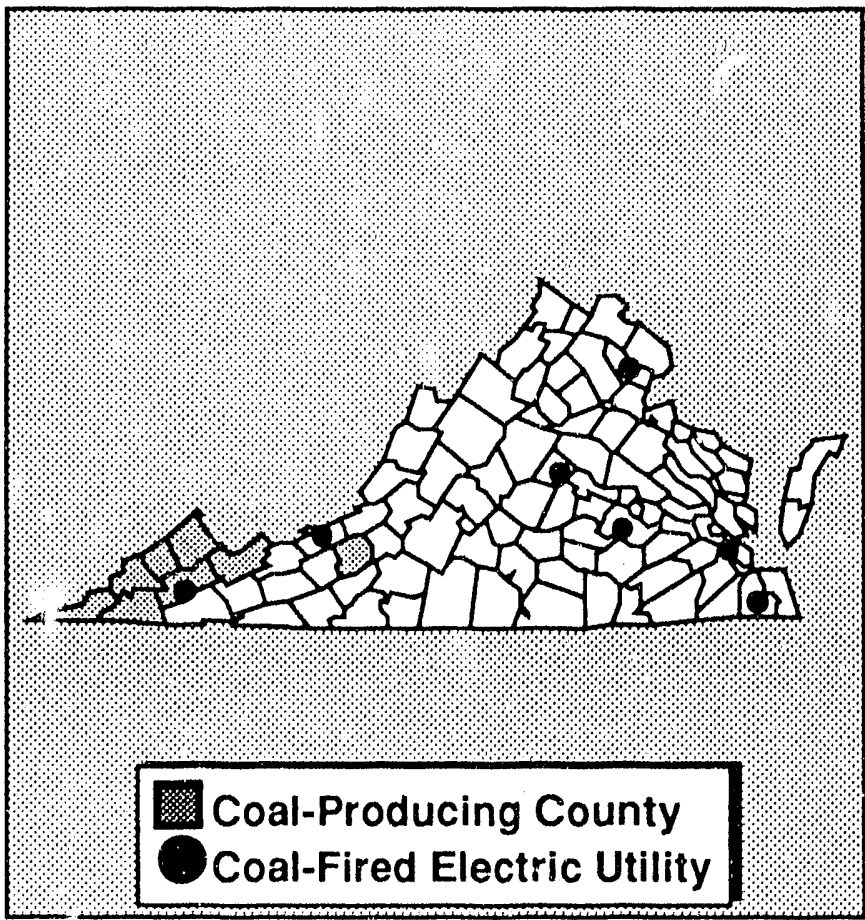

Table B29. Virginia Coal Facts

\begin{tabular}{|c|c|c|c|c|c|c|c|}
\hline & 1970 & 1975 & 1980 & 1986 & 1987 & 1988 & 1889 \\
\hline 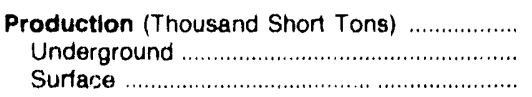 & $\begin{array}{r}35,016 \\
28,018 \\
6,998\end{array}$ & $\begin{array}{l}35,510 \\
23,181 \\
12,32 \theta\end{array}$ & $\begin{array}{r}41,009 \\
32,358 \\
8,651\end{array}$ & $\begin{array}{r}40,940 \\
33,550 \\
7,390\end{array}$ & $\begin{array}{r}44,543 \\
36,943 \\
7,589\end{array}$ & $\begin{array}{r}46,886 \\
37,809 \\
8,076\end{array}$ & $\begin{array}{r}43,006 \\
36,044 \\
6,962\end{array}$ \\
\hline Average Mine Price (Dollars per Short Ton). & 7.03 & 30.46 & 34.58 & 30.16 & 27.42 & 26.49 & 27.04 \\
\hline Total Value of Production (Million Dollars) ... & 246 & 1,082 & 1,418 & 1,235 & 1,221 & 1,215 & 1,189 \\
\hline Percentage of U.S. Coal Production ............. & 5.7 & 5.4 & 4.8 & 4.6 & 4.8 & 4.8 & 4.4 \\
\hline Natlonal Pank (Production) & 6 & 6 & 6 & 7 & 7 & 7 & 7 \\
\hline $\begin{array}{l}\text { Total U.S. Coal Ploduction } \\
\text { (Thousand Short Tons) }\end{array}$ & 612,661 & 654,641 & 829,700 & 883,638 & 918,762 & 950,285 & 980,729 \\
\hline 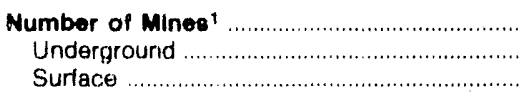 & $\begin{array}{l}803 \\
566 \\
237\end{array}$ & $\begin{array}{l}745 \\
374 \\
371\end{array}$ & $\begin{array}{l}502 \\
387 \\
115\end{array}$ & $\begin{array}{l}509 \\
390 \\
11\end{array}$ & $\begin{array}{l}177 \\
343 \\
134\end{array}$ & $\begin{array}{l}413 \\
304 \\
109\end{array}$ & $\begin{array}{r}365 \\
271 \\
94\end{array}$ \\
\hline $\begin{array}{l}\text { Employment } \\
\text { Underground } \\
\text { Surface }\end{array}$ & $\begin{array}{r}10,630 \\
9,567 \\
1,063\end{array}$ & $\begin{array}{r}16,510 \\
13,360 \\
3,150\end{array}$ & $\begin{array}{r}15,876 \\
13,569 \\
2,307\end{array}$ & $\begin{array}{r}13,268 \\
11,553 \\
1,715\end{array}$ & $\begin{array}{r}12,047 \\
10,22.8 \\
1,819\end{array}$ & $\begin{array}{r}10,698 \\
9,046 \\
1,650\end{array}$ & $\begin{array}{r}10,371 \\
8,889 \\
1,482\end{array}$ \\
\hline 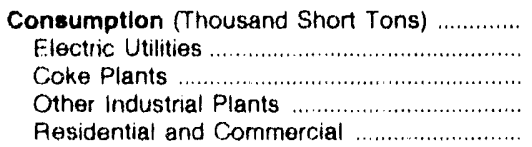 & $\begin{array}{r}11,085 \\
6,661 \\
22 \\
4,38 \% \\
(4)\end{array}$ & $\begin{array}{r}6,561 \\
3,989 \\
0 \\
2,572 \\
(4)\end{array}$ & $\begin{array}{l}9,281 \\
5,560 \\
3856 \\
2,875 \\
(\mathbf{3})\end{array}$ & $\begin{array}{r}11,656 \\
7,166 \\
31,155 \\
3,334 \\
(3)\end{array}$ & $\begin{array}{r}13,227 \\
8,297 \\
1,267 \\
3,662 \\
\left({ }^{3}\right)\end{array}$ & $\begin{array}{r}13,430 \\
8,468 \\
31,216 \\
3,745 \\
(3)\end{array}$ & $\begin{array}{r}84,279 \\
9,573 \\
1,130 \\
3,576 \\
(3)\end{array}$ \\
\hline
\end{tabular}

1 For 1980 , includes only mines that produced 10,000 or more tons.

2 Beginning in 1980 , includes only mines that produced 10,000 or more tons.

y Consumption daia at Coke Plants and in Residential and Commercial Sector are combined to avoid disclosure of individual company data.

4 Consumption data at Other Industrial Plants and in Residential and Commercial Sector are combined to avoid disclosure of individual company data. Note: Total may not equil sum of components because of independent rounding.

Sources: Energy Informition Administration, Caal Production; Quarterty Coal Report; Woekly Coal Production; Coal Data: A Reterence; Eloctric Power Monthly 1989; and Inventory of Power Plants in the United States 1989; Bureau of Mines, U.S. Department of interior, Minerals Yearbook; Information Circular 8705: Histonical Fug/s and Energy Consumption Data, 1960-72, United States by States and Census Districts West of the Mississippi; and Geological Survey Bulletin 1412: Cavy Fesources of the United States, January 1, 1974. 


\section{Coal Highlights}

Production

(thousand short tons)

Value

(million dollars)

Number of Employees

Consumption

(thousand short tons)

Coal-Fired Electric Generating Units

Number

Summer Capability (megawatts)

$\%$ of Total Summer Capability

Generation (gigawatthours)

Coal Demsits

Area (square miles)

Percent of State Land

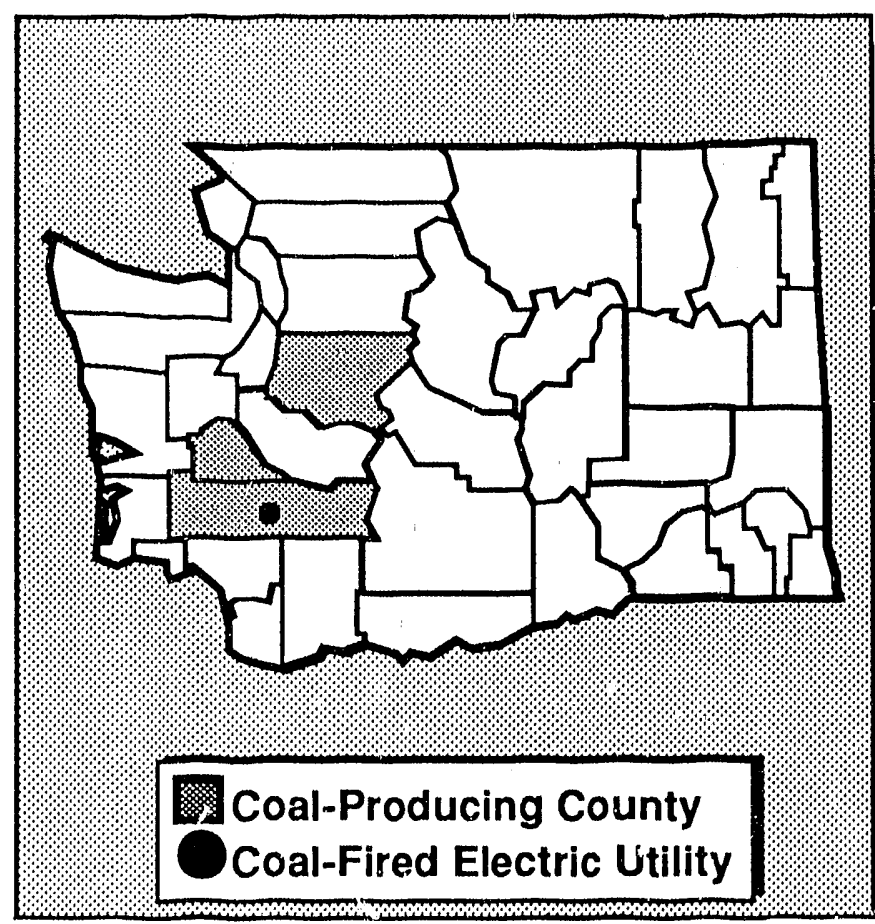

Table B30. Washington Coal Facts

\begin{tabular}{|c|c|c|c|c|c|c|c|}
\hline$\ldots$ & 1970 & 1975 & 1980 & 1985 & 1987 & 1988 & 1989 \\
\hline Production (Thousand Short Tons) ..................... & 37 & 3,743 & 5,140 & 4,438 & 4,449 & 5,170 & 5,039 \\
\hline 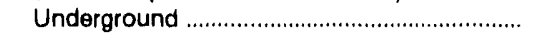 & 32 & 13 & & & 0 & 0 & 0 \\
\hline 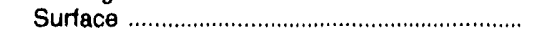 & 5 & 3,730 & 5,140 & 4,438 & 4,449 & 5,170 & 5,039 \\
\hline Averagu Mine Price (Dollars per Short Ton) & 12.81 & w & $w$ & $w$ & $w$ & $w$ & w \\
\hline Total Value of Produstion (Million Dollars) ... & .5 & $w$ & w & $w$ & $w$ & w & w \\
\hline Percentage of U.S. Coal Production .............. & (') & .6 & .6 & .5 & .5 & .5 & .5 \\
\hline Wational Rank (Production) ................................... & 24 & 19 & 20 & 19 & 18 & 18 & 18 \\
\hline $\begin{array}{l}\text { Total U.S. Coal Production } \\
\text { (Thousand Short Tons) }\end{array}$ & 612,661 & 654,641 & 829,700 & 883,638 & 918,762 & 950,265 & 980,729 \\
\hline Number of Mines' & 3 & 4 & 2 & 3 & 4 & 4 & 4 \\
\hline 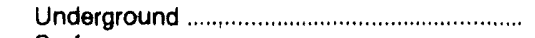 & 2 & 1 & 0 & 0 & 0 & 0 & 0 \\
\hline Surface & 1 & 3 & 2 & 3 & 4 & 4 & 4 \\
\hline Employment's & 30 & 520 & 597 & 677 & 699 & 716 & 787 \\
\hline 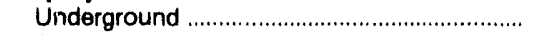 & 28 & 10 & 0 & 0 & 0 & 0 & 0 \\
\hline Surface & 2 & 510 & 597 & 677 & 699 & 716 & 787 \\
\hline Conzumption (Thousand Short Tons) .............. & 374 & 4,121 & 5,443 & 5,616 & 5,819 & 5,929 & 5,843 \\
\hline 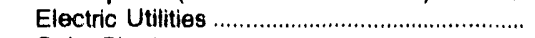 & 0 & 3,717 & 4,950 & 5,192 & 5,468 & 5,561 & 5,514 \\
\hline Coke Plants & $c$ & 0 & 0 & 0 & 0 & 0 & 0 \\
\hline Other Industrial Plants & 296 & 4404 & 332 & 208 & 298 & 252 & 238 \\
\hline 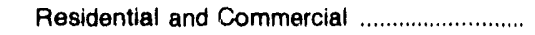 & 78 & (4) & 161 & 216 & 52 & 116 & 90 \\
\hline
\end{tabular}

1 Less than 0.05 percent.

2 For 1980 , includes only mines that produced 10,000 or more tons

3 Beginning in 1980, includes only mines that produced 10,000 or more tons.

- Consumption data at Other Industriai Plants and in Residential and Commercial Sector are combined to avoid disclosure of individual company data.

- Withheld to avoid disclosure of individual company data.

Note: Total may not equal sum of components because of independent rounding.

Sources: Energy Information Administration, Coal Production; Quarterly Coal Report; Weekly Coal Production; Coal Data: A Reference; Electric Power Monthly 1989; and Inventory of Power Plants in the United States 1989; Bureau of Mines, U.S. Department of interior, Minerals Yeartook; Informa. tion Circular 8705: Historical Fuels and Energy Consumption Data, 1960-72, United States by States and Census Districts West of the Mississippi; and Geological Survey Bulletin 1412: Coal Resources of the United States, January 1, 1974. 


\section{Coal Highlights}

Production

(thousand short tons)

Value

(million dollars)

Number of Employees

Consumption

(thousand short tons)

\section{Cual-Fired Electric Generating Units}

Number

Summer Capability (megawatts)

\% of Total Summer Capability

Generation (gigawatthours)

Coal Deposits

Area (square miles)

Percent of State Land
$1.53,580$

29,482

37,186

14,315

82,105

16,800

69

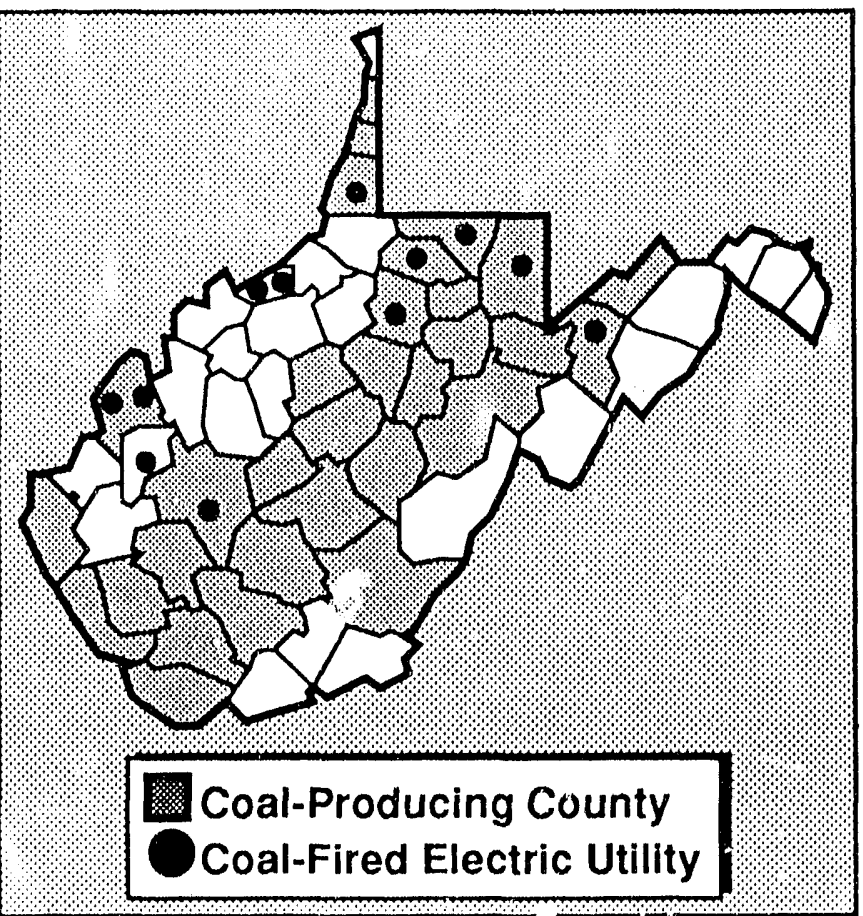

Table B31. West Virginia Coal Facts

\begin{tabular}{|c|c|c|c|c|c|c|c|}
\hline & 1970 & 1975 & 1980 & 1985 & 1987 & 1988 & 1989 \\
\hline Production (Thousand Short Tons) ................... & 144,072 & 109,283 & 121,584 & 127,764 & 136,676 & 145,005 & 153,560 \\
\hline 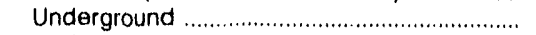 & 116,414 & 88,357 & 96,409 & 103,753 & 107,463 & 110,076 & 113,202 \\
\hline Surface & 27,657 & 20,926 & 25,175 & 24,011 & 29,213 & 34,828 & 40,378 \\
\hline Averege Mine Price (Dollars per Short Ton) & 7.93 & 29.35 & 34.88 & 33.47 & 29.15 & 28.22 & 28.69 \\
\hline Total Value of Production (Million Dollars) ... & 1,142 & 3,207 & 4,241 & 4,276 & 3,984 & 4,092 & 4,406 \\
\hline Percentage of U.S. Coal Production ............. & 23.5 & 16.7 & 14.7 & 14.5 & 14.9 & 15.3 & 15.7 \\
\hline National Rank (Production) ………….................... & 1 & 2 & 2 & 3 & 3 & 3 & 3 \\
\hline $\begin{array}{l}\text { Total U.S. Coal Production } \\
\text { (Thousand Short Tons) }\end{array}$ & 612,661 & 654,641 & 829,700 & 883,638 & 918,762 & 950,265 & 980,728 \\
\hline Number of Mines' & 1,319 & 1,072 & 762 & 797 & 778 & 747 & 773 \\
\hline 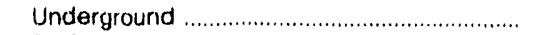 & 748 & 703 & 539 & 502 & 478 & 477 & 461 \\
\hline Surtace & 571 & 368 & 223 & 295 & 300 & 270 & 312 \\
\hline (1) & 47,144 & 54,210 & 53,403 & 36,658 & 29,458 & 28,972 & 28,482 \\
\hline 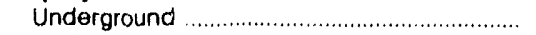 & 41,323 & 48,400 & 45,904 & 31,106 & 24,513 & $23,44 \%$ & 23,048 \\
\hline Surface & 5,821 & 5,810 & 7,499 & 5,552 & 4,945 & 5,471 & 6,434 \\
\hline Consumption (Thousand Short Tons) .............. & 24,395 & 34,360 & 34,673 & 34,907 & 34,815 & $36 \cdot 35$ & 37,186 \\
\hline Electric: Utilities & 14,320 & 26,320 & 28,499 & 31,367 & 30,605 & 31,703 & 32,391 \\
\hline 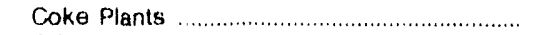 & 5,074 & 4,467 & 3,851 & 31,454 & 31,884 & 32,135 & 32,084 \\
\hline Other Industrial Plants & 5,001 & 4,573 & 2,223 & 2,086 & 2,326 & 2,597 & 2,712 \\
\hline 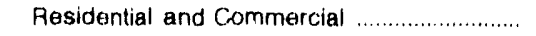 & $(4)$ & $(4)$ & $\left({ }^{3}\right)$ & $\left({ }^{3}\right)$ & $\left({ }^{3}\right)$ & (3) & $\left({ }^{3}\right)$ \\
\hline
\end{tabular}

1 For 1980 , includes only mines that produced 10,000 or more tons

2 Beginning in 1980 , includes only mines that produced 10,000 or more tons.

3 Consumption data at Coke Plants and in Hesidential and Commercial Sector are combined to avoid disclosure of individual company data.

4 Consumption data at Other Industrial Plants and in Residertial and Commercial Sector are combined to avoid disclosure of individual company data Note: Total may not equal sum of components because of independent rounding

Sources: Energy Information Administration, Coal Production; Ouarterly Coal Report; Wegkly Coal Production: Coal Data: A Reterencts: Electric Power Monthly 1989; and Inventory of Power Plants in the United States 1989: Bureau of Mines, US. Department of Interior, Minerals Yearbook: Informa. tion Circular 8705: Historical Fuels and Energy Consumption Data, 1960-72, United States by States and Consus Districts West of the Mississippi; and Geo. logical Survey Bulletin 1412: Coal Hesources of the United States, January 1, 1974 


\section{Coal Highlights}

Production

(thousand short tons)

171,558

Value

(million dollars)

Number of Employees

Consumption

(thousand short tons)

Coal-Fired Electric Generating Units

Number

Summer Capability (megawatts)

$\%$ of Total Summer Capability

Generation (gigawatthours)

Coal Deposits

Area (square miles)

Percent of State Land

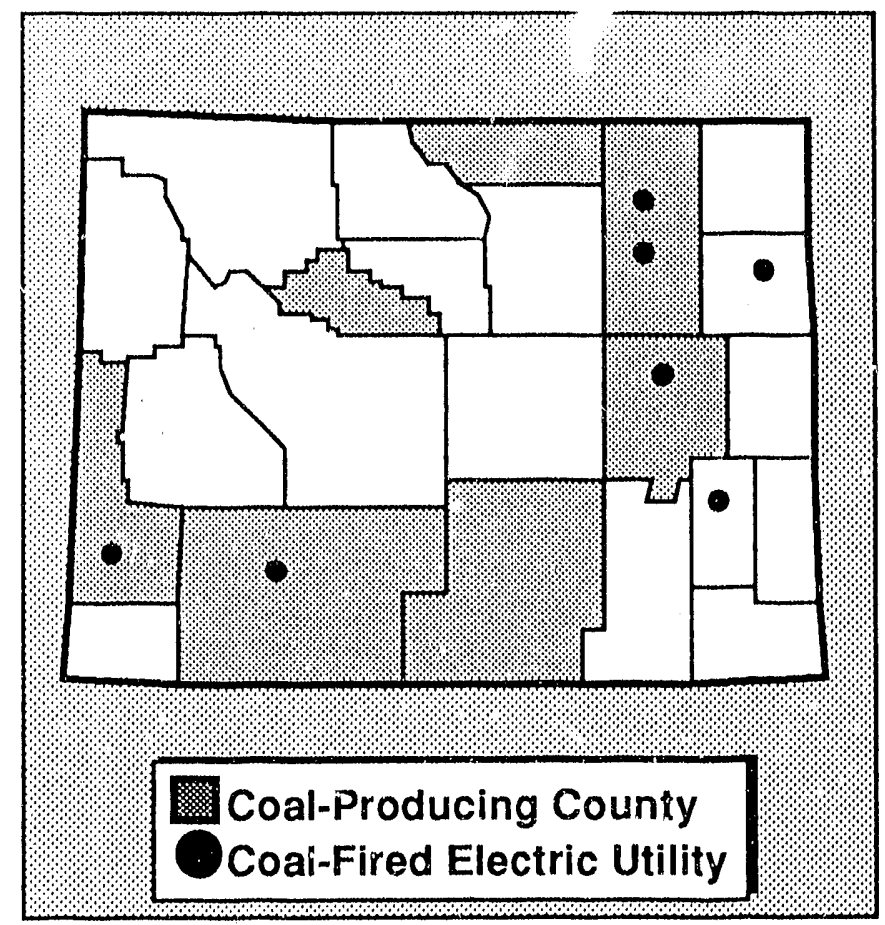

Table B32. Wyoming Coal Facts

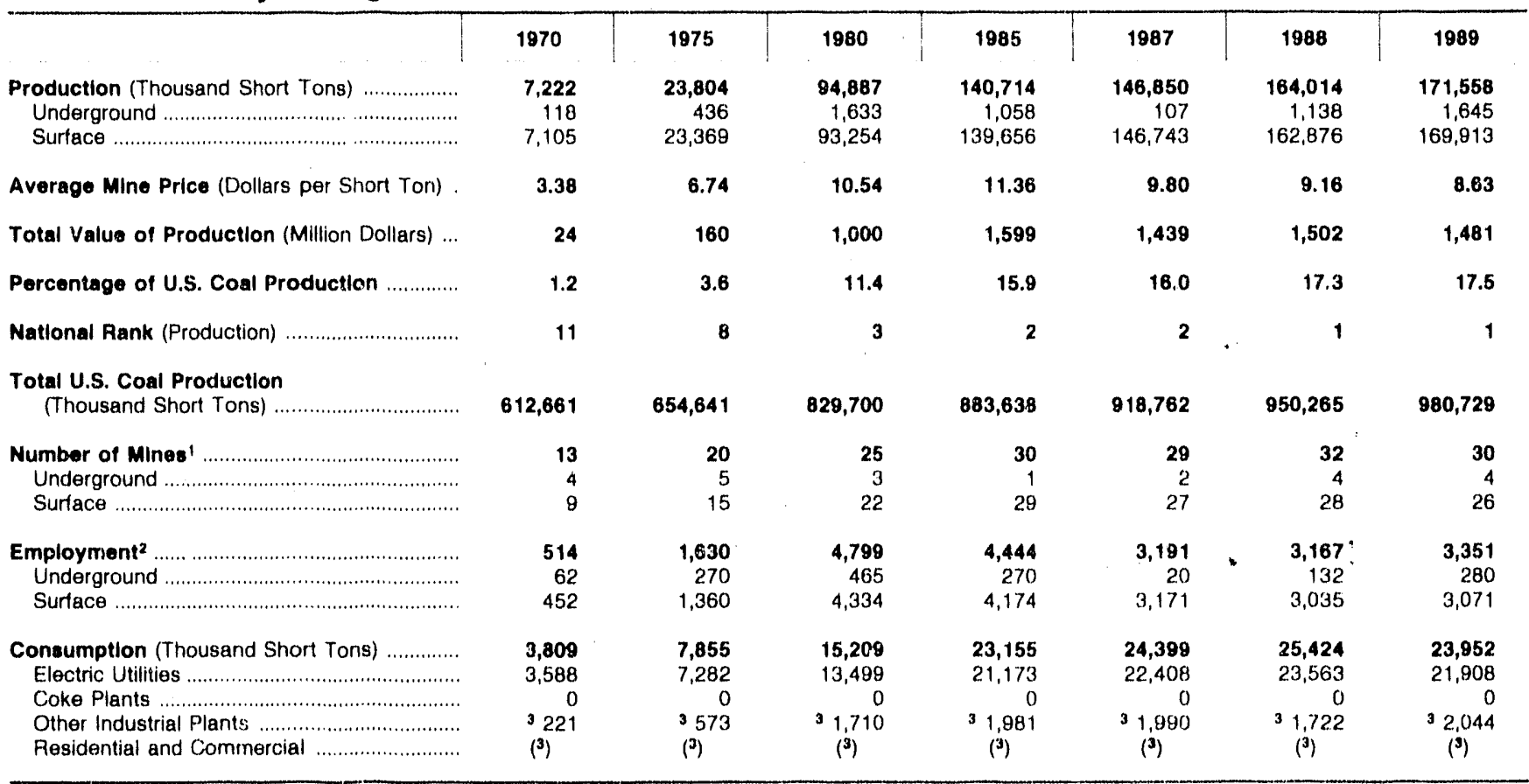

1 For 1980 , includes only mines that produced 10,000 or more toris

2 Beginning in 1980, includes only mines that produced 10,000 or more tons.

3 Consumption data at Other Industrial Plants and in Residential and Commercial Sector are combined to avoid disclosure of individual company data. Note: Total may not equal surn of components because of independent rounding.

Sources: Energy Iriformation Administration, Coal Production; Quarterly Coal Repon; Weekly Coal Production; Coal Data: A Reference; Eloctric Power Monthly 1989; and Inventory of Power Plants in the United States 1989; Bureau of Mines, U.S. Department of Interior, Minorals Yeartook; Informa tion Circular 8705: Historical Fuels and Energy Consumption Data, 1960.72, United States by States and Census Districts West of the Mississippi; and Geological Survey Bulletin 1412: Cos/ Resources of the United States, Jamuary i, 1974. 


\section{Appendix C}

\section{Reference Tables and Maps}

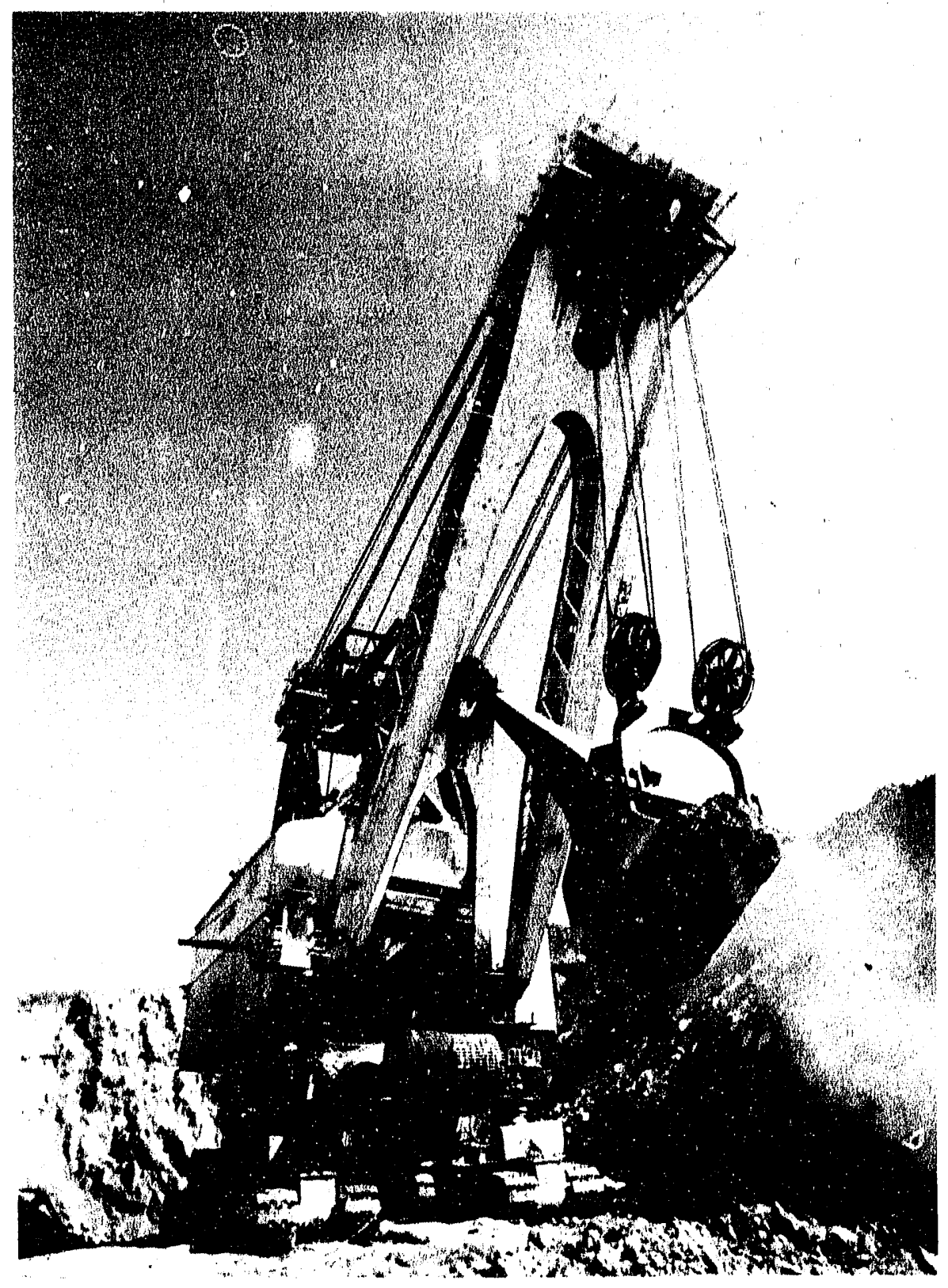

At an Ohio surface mine, a giant, shovel-type earth mover (GEM) removes overburden. 


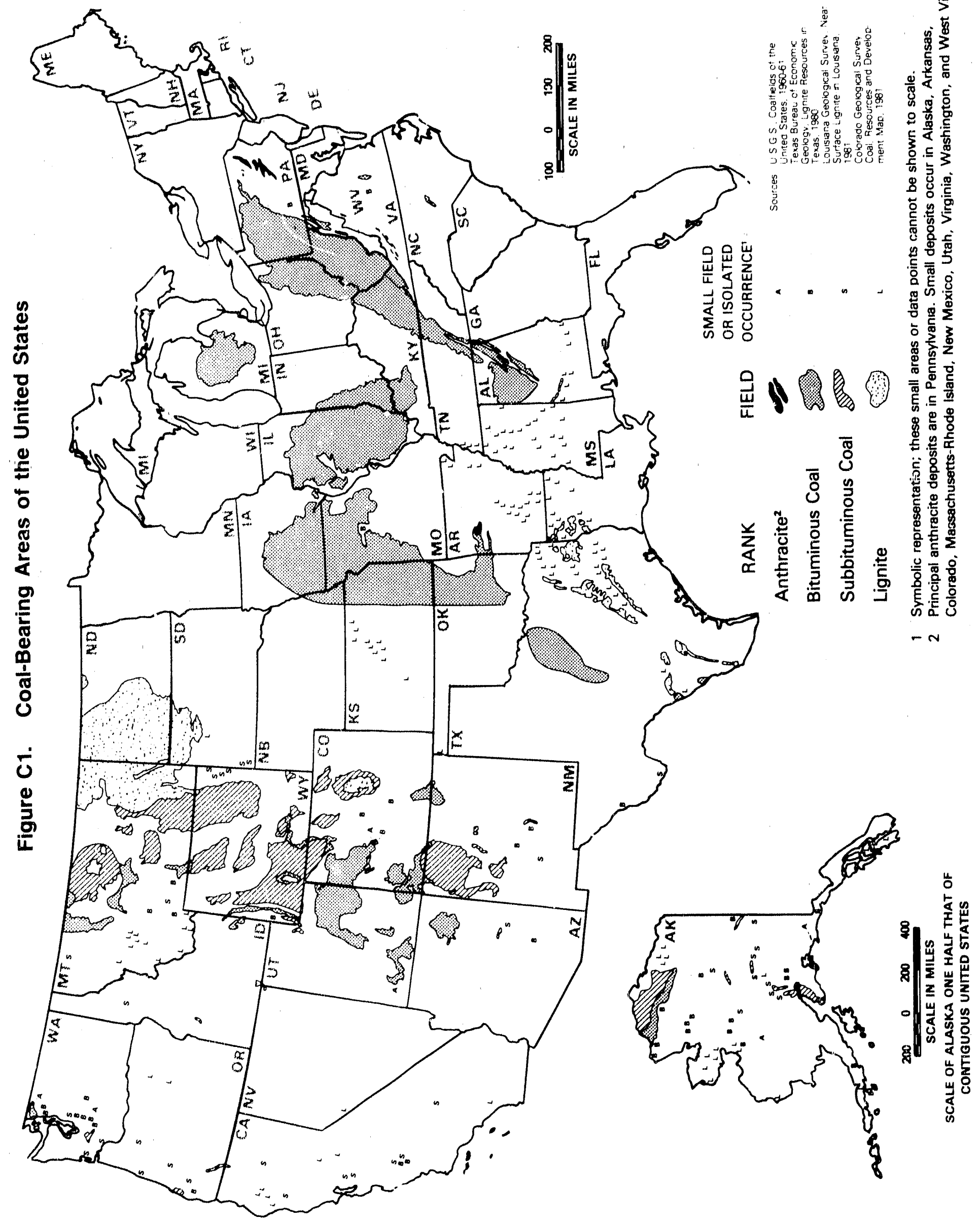


Figure C2. Coal-Producing Districts

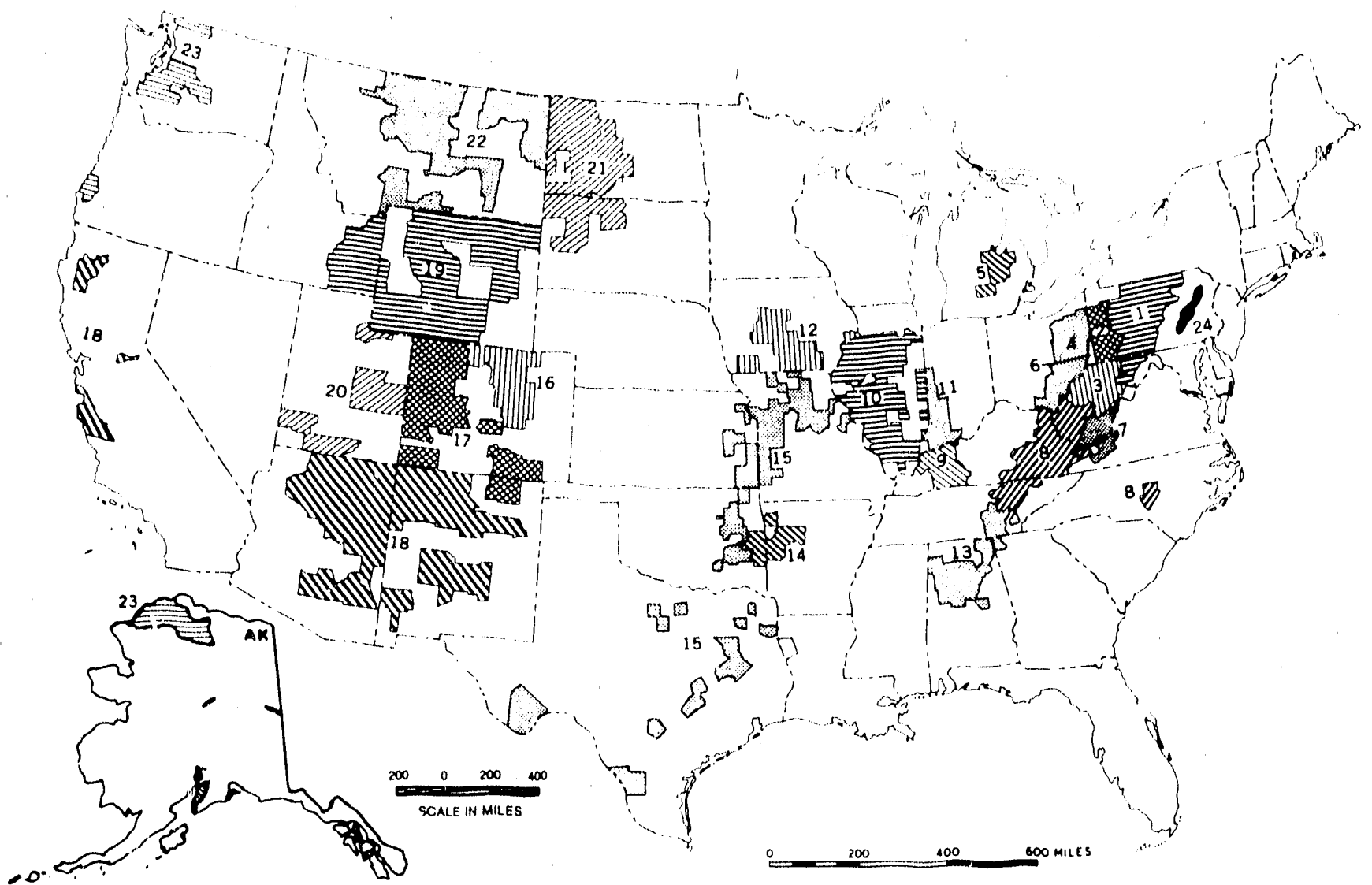

Bituminous Coal Producing Districts as Detined in the Bituminous Coal Act of 1937 and Amendments

The districts were originally established to aid in formulating minirnum prices of bituminous coal and lignite. Because much statistical intormattion was complled in terms of these districts, their use for statistical purposes hás continued since the abandonment of that legislation in 1943.

District 24 is the anthiacite producing district in Pennsylvania 
Coal-Producing Districts

\section{District 1}

Maryland - All mines in the State.

Pennsylvania - All mines in the following counties: Bedford, Blair, Bradford, Cambria, Cameron, Cintre, Clarion, Clearfield, Clinton, Elk, Forest, Fulton, Huntingdon, Jefferson, Lycoming, McKean, Mifflin, Potter, Somerset, and Tioga. Selected mines in the following counties: Armstrong County (part), all mines east of the Allegheny River, and those mines served by the Pittsburgh and Shawmut Railroad located on the west bank of the river; Fayette County (part), all mines located on and east of the line of Indian Creek Valley branch of the Baltimore \& Ohio Railroad (CSX Transportation); Indiana County (part), all mines not served by the Saltsburg branch of the Consolidated Rail Corporation; and Westmoreland County (part), all mines served by the Consolidated Rail Corporation from Torrance, east.

West Virginia - All mines in the following counties: Grant, Mineral, and Tucker.

\section{District 2}

Pennsylvania - All mines in the following counties: Allegheny, Beaver, Butler, Greene, Lawrence, Mercer, Venango, and Washington. Selected mines in the following counties: Armstrong County (part), all mines west of the Allegheny River except those mines served by the Pittsburgh \& Shawmut Railroad; Fayette County (part), all mines except those on and east of the line of Indian Creek Valley branch of the Baltimore \& Ohio Railroad (CSX Transportation);Indiana County (part), all mines served by the Saltsburg branch of the Consolidated Rail Corporation; and Westmoreland County (part), all mines except those served by the Consolidated Rail Corporation from Torrance, east.

\section{District 3}

West Virginia - All mines in the following counties: Barbour, Braxton, Calhoun, Doddridge, Gilmer, Harrison, Jackson, Lewis, Marion, Monongalia, Pleasants, Preston, Randolph, Ritchie, Roane, Taylor, Tyler, Upshur, Webster, Wetzel, Wirt, and Wood. Selected mines in the following county: Nicholas County (part), all mines served by or north of the Baltimore \& Ohio Railroad (CSX Transportation).

\section{District 4}

Ohio All mines in the State.

\section{District 5}

Michigan - All mines in the State.

\section{District 6}

West Virginia - All mines in the following counties: Brooke, Hancock, Marshall, and Ohio.

\section{District 7}

Virginia - All mines in the following counties: Craig, Giles, Montgomery, Pulaski, and Wythe. Selected mines in the following counties: Buchanan County (part), all mines in that portion of the county served by the Richlands-Jewell Ridge branch of the Norfolk \& Western Railroad (Norfolk Southern Corporation) and in that portion on the headwaters of Dismal Creek east of Lynn Camp Creek (a tributary of Dismal Creek); and Tazewell County (part), all mines in those portions of the county served by the Dry' Fork branch to Cedar Bluff and from Bluestone Junction to Boissevain branch of the Norfolk \& Western Railroad (Norfolk Southern Corporation) and Richlands-Jewell Ridge branch of the Norfolk \& Western Railroad (Norfolk Southern Corporation).

West Virginia - All mines in the following counties: Greenbrier, Mercer, Monroe, Pocahontas, and Summers. Selected mines in the following counties: Fayette County (part), all mines east of Gauley River and all mines served by the Gauley River branch of the Chesapeake \& Ohio Railroad (CSX Transportation) and mines served by the Norfolk \& W'estern Railroad (Norfolk Southern Corporation); McDowell County (part), all mines in that portion of the county served by the Dry Fork branch of the Norfolk \& Western Railroad (Norfolk Southern Corporation) and east thereof; Raleigh County (part), all mines except those on the Coal River branch of the Chesapeake \& Ohio Railroad (CSX Transportation) and north thereof; and Wyoming County (part), all mines in that portion served by the Guyandot branch of the Norfolk \& Western Railroad (Norfolk Southern Corporation) lying east of the mouth of Skin Fork of Guyandot River and in that portion served by the Virginia division main line of the Norfolk \& Western Railroad (Norfolk Southern Corporation). 


\section{District 8}

Kentucky - All mines in the following counties in eastern Kentucky: Bell, Boyd, Breathitt, Carter, Clay, Clinton, Elliott, Estill, Floyd, Greenup, Harlan, Jackson, Johnson, Knott, Knox, Laurel, Lawrence, Lee, Leslie, Letcher, McCreary, Magoffin, Martin, Morgan, Owsley, Perry, Pike, Powell, Pulaski, Rockcastle, Wayne, Whitley, and Wolfe.

North Carolina - All mines in the State.

Tennessee - All mines in the following counties: Anderson, Campbell, Claiborne, Cumberland, Fentress, Morgan, Overton, Roane, and Scott.

Virginia - All mines in the following counties: Dickinson, Lee, Russell, Scott, and Wise. Selected mines in the following counties: Buchanan County (part), all mines in the county, except in that portion on the neadwaters of Dismal Creek, east of Lynn Camp Creek (a tributary of Dismal Creek) and in that portion served by the Richlands-Jewell Ridge branch of the Norfolk \& Western Railroad (Norfolk Southern Corporation); and Tazewell County (part), all mines in the county except in those portions served by the Dry Fork branch of the Norfolk \& Western Railroad (Norfolk Southern Corporation) and branch from Bluestone Junction to Boissevain of Norfolk \& Western Railroad (Norfolk Southern Corporation) and Richlands-Jewell Ridge branch of the Norfolk \& Western Railroad (Norfolk Southern Corporation).

West Virginia - All mines in the following counties: Boone, Cabell, Clay, Kanawha, Lincoln, Logan, 1son, Mingo, Putnam, and Wayne. Selected mines in the following counties: Fayette County (part), all mines west of the Gauley River except mines served by the Gauley River branch of the Chesapeake \& Ohio Railroad (CSX Transportation); McDowell County (part), all mines west of and not served by the Dry Fork branch of the Norfolk \& Western Railroad (Norfolk Southern Corporation); Nicholas County (part), all mines in that part of the county south of and not served by the Baltimore \& Ohio Railruad (CSX Transportation); Raleigh County (part), all mines on the Coal River branch of the Chesapeake \& Ohio Railroad (CSX Transportation) and north thereof; and Wyoming Wyoming County (part), all mines in that portion served by the Guyandot branch of the Norfolk \& Western Railroad (Norfolk Southern Corporation) and lying west of the mouth of Skin Fork of Guyandot River.

\section{District 9}

Kentucky - All mines in the following counties in western Kentucky: Butler, Caldwell, Christian, Crittenden, Daviess, Edmonson, Grayson, Hancock, Henderson,
Hopkins, Logan, McLean, Muhlenberg, Ohio, Simpson, Todd, Union, Warren, and Webster.

\section{District 10}

Illinois - All minf s in the State.

\section{District 11}

Indiana - All mines in the State.

\section{District 12}

Iowa - All mines in the State.

\section{District 13}

Alabuma - All mines in the State.

Georgig - All mines in the State.

Tennessee - All mines in the following counties: Bledsoe, Grundy, Hamilton, Marion, McMinn, Rhea, Sequatchie, Van Buren, Warren, and White.

\section{District 14}

Arkansas - All mines in the State.

Oklahoma - All mines in the following counties: Haskell, Le Flore, and Sequoyah.

\section{District 15}

Kansas - All mines in the State.

Louisiana - All mines in the State.

Missouri - All mines in the State.

Oklahoma - All mines in the following counties: Coal, Craig, Latimer, McIntosh, Muskogee, Nowata, Okmulgee, Pittsburgh, Rogers, Tulsa, and Wagoner.

Texas - All mines in the State. 


\section{District 16}

Colorado - All mines in the following counties: Adams, Arapahoe, Boulder, Douglas, Elbert, El Paso, Jackson, Jefferson, Larimer, and Weld.

\section{District 17}

Colorado - All mines except those included in District 16.

New Mexico - All mines except those included in District 18.

\section{District 18}

Arizona - All mines in the State.

California - All mines in the State.

New Mexico - All mines in the following counties: Catron, Grant, Lincoln, McKinley, Rio Arriba, Sandoval, San Juan, San Miguel, Santa Fe, and Socorro.

\section{District 19}

Idaho - Al: mines in the State.

Wyoming - All mines in the State.

\section{District 20}

Utah - All mines in the State.

\section{District 21}

North Dakota - All mines in the State.

South Dakcta - All mines in the State.
District 22

Montana - All mines in the State.

\section{District 23}

Alaska - All mines in the State.

Oregon - All mines in the State.

Washington - All mines in the State.

\section{District 24 (Pennsylvania Anthracite)}

Pennsylvania - All mines in the following counties: Carbon, Columbia, Dauphin, Lackawanna, Lebanon, Luzerne, Northumberland, Schuylkill, Snyder, Sullivan, and Susquehanna. All anthracite mines in Bradford County.

\section{Coal-Producing Regions}

\section{Appalachian}

Alabama, Georgia, Eastern Kentucky, Maryland, Ohio, Pennsylvania, Tennessee, Virginia, IVest Virginia.

\section{Interior}

Arkansas, Illinois, Indiana, Iowa, Kansas, Western Kentucky, Louisiana, Missouri, Oklahoma, Texas.

\section{Western}

Alaska, Arizona, California, Colorado, Montana, New Mexico, North Dakota, Utah, Washington, Wyoming. 
Principles

The lower rank coals can be classified based on heal content. The heat content of the higher rank coals is generally above 14,000 Btu per pound for each coal rank group (except for meta-anthracite, which trends slightly lower), and heat content ranges vary within a relatively narrow range. Since heat content is not a dependable criterion for these higher rank coals, their rank categories are instead described by degree of metamorphism. or "coalification," a property that is measured by fixed carbon content. Finally, the agglomerating usaracter of bituminous coals is a critical attribute for certain coal consumers, and thus agglomerating character has come tu define the distinctions between certain adjacent coal groups. Some high-volatile $\mathrm{C}$ bituminous and subbituminous $\mathrm{A}$ coals can be distinguished only on the basis of agglomerating character.

\begin{tabular}{|c|c|c|c|c|}
\hline Coal Rank & Coal Group & \multicolumn{3}{|c|}{ Basis of Classification } \\
\hline \multirow{2}{*}{\multicolumn{2}{|c|}{ Coals Classified by Fixed Carbon }} & \multicolumn{2}{|c|}{ Fixed Carbon Percentage 1} & \multirow[b]{2}{*}{$\begin{array}{c}\text { Agglomerating } \\
\text { Character }\end{array}$} \\
\hline & & $\begin{array}{l}\text { Equal to or } \\
\text { Greater than }\end{array}$ & Less than & \\
\hline \multirow[t]{3}{*}{ 1. Anthracitic } & 1. Meta-anthracite & 98 & $-\cdot$ & Non-agglomerating \\
\hline & 2. Anthracite & 92 & 98 & Non-agglomerating \\
\hline & 3. Semianthracite 2 & 86 & 92 & Non-agglonıerating \\
\hline \multirow[t]{4}{*}{ II. Bituminous } & 1. Low-volatile bituminous & 78 & 86 & Commonly agglomerating 3 \\
\hline & 2. Medium-volatile bituminous & 469 & 78 & Commonly agglomerating 3 \\
\hline & 3. High-volatile $A$ bituminous & -. & 465 & Commonly agglomerating 3 \\
\hline & & Heat Content & per Pound 5 & \\
\hline \multicolumn{2}{|c|}{ Coals Classified by Heat Content } & $\begin{array}{l}\text { Equal to or } \\
\text { Greater than }\end{array}$ & Less than & \\
\hline \multirow[t]{3}{*}{ Il. Bituminous } & 4. High-volatile 13 bituminous & 13,000 & 14,000 & Commonly agglomerating 3 \\
\hline & 5. High-volatile $C$ bituminous & 11,500 & 13,000 & Cornmonly agglomerating 3 \\
\hline & 6. High-volnille $\mathrm{C}$ bituminous & 10,500 & 11,500 & Agglomerating \\
\hline \multirow[t]{3}{*}{ III. Subbitumnious } & 1. Subbituminous A & 10,500 & 11,500 & Non-agglomerating \\
\hline & 2. Subrituminous B & 9,500 & 10,500 & Non-agglomerating \\
\hline & 3. Si;obituminous $\mathrm{C}$ & 8,300 & 9,500 & Non-agglomerating \\
\hline \multirow[t]{2}{*}{ IV. Lignitic } & 1. Lignite $A$ & 6,300 & 8,300 & Non-agglomerating \\
\hline & 2. Lignite $B$ & .. & 6,300 & Non-agglomerating \\
\hline
\end{tabular}

1 Percentages are based on dry mineral-matter-free coal. Volatile matter (not shown) is the complement of fixed carbon; that is, the percentages of fixed carbon and volatile matter sum to 100 percent. As fixed carbon percentage decreases, therefore, volatile matter percentage increases by the same amount.

2 If agglomerating, classify in low-volatile group of the bituminois class.

3 There may be nonagglomerating varieties in the bituminous class, most notably in the high-volatile $C$ bituminous group.

4 Coals having 69 percent or more fixed carbon are classified accorcing to fixed carbon, regardless of Btu value. Coals with less than 69 percent fixed carbon. but with 14,000 or more Btu per pound, are classified as high-volatile A bituminous.

5 Calorific values in Btu per pound, on a moist mineral-matter-free basis.

Note: Terms in this table are defined in the Glossary.

Source: Adapted from American Society for Testing and Materials 1988, Standard Classification of Coal by Rank, ASTM Designation D 388.84 


\section{Appendix D}

\section{Explanatory Notes}

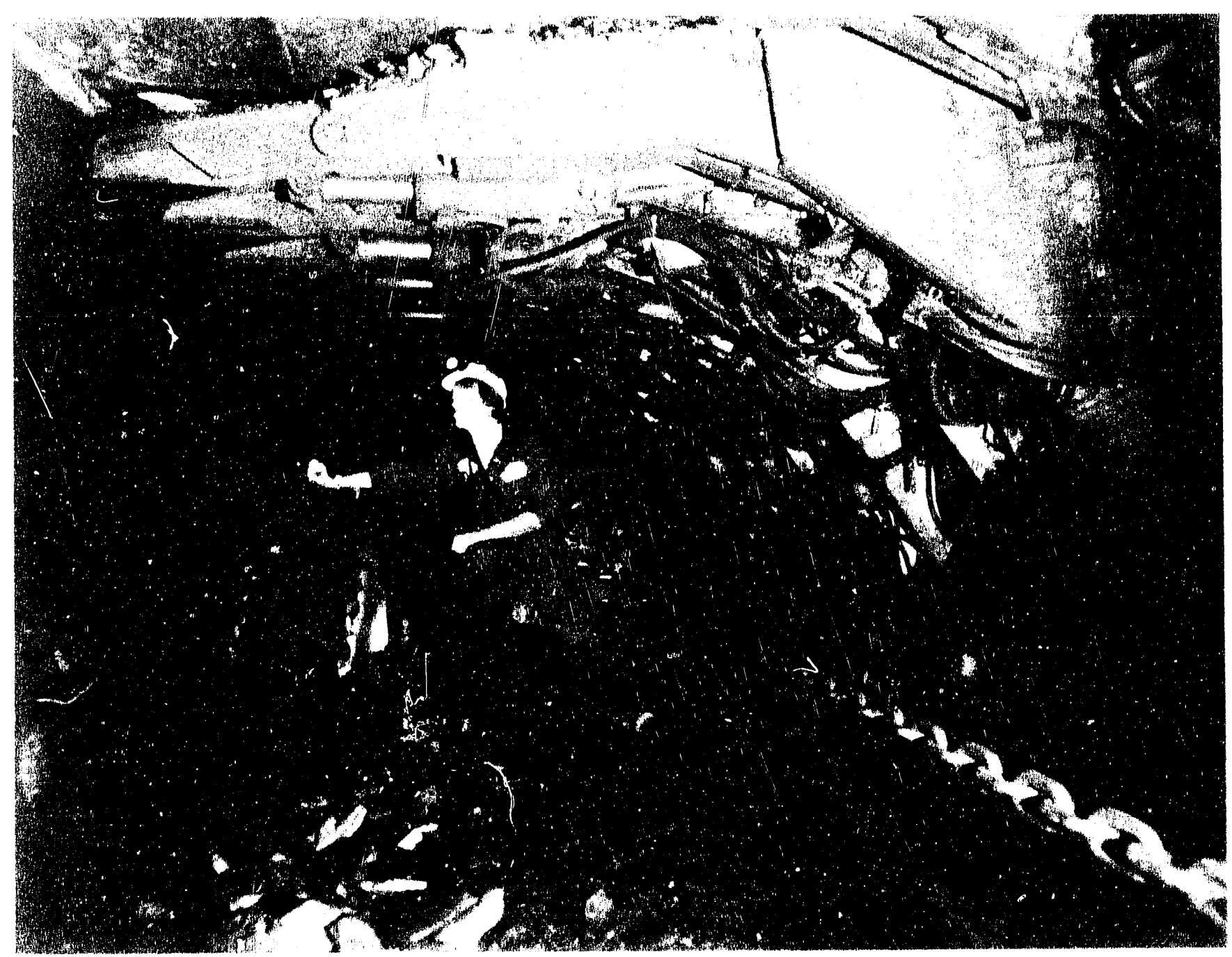

Miners are protected during longwall operations by hydraulically-operated, canopied roof supports. 


\section{Appendix D}

\section{Explanatory Notes}

\section{Coal Production}

\section{Sources of Data}

As early as the 1880 's, the U.S. Geological Survey began collecting coal data under a voluntary reporting system. The responsibility for gathering this information was transferred to the Bureau of Mines in the 1920 's, initially under the U.S. Department of Commerce and later under the U.S. Department of the Interior, which published the data in its Minerals Yearbook. Except for a brief period from 1937 to 1943, when bituminous coal data ware collected under the mandatory authority of the Bituminous Coal Act, the Bureau of Mines continued to conduct voluntary coal surveys until the Department of Energy was created.

The Energy Information Administration (EIA) began collecting annual coal production data on October 1, 1977. The 1989 coal production and related data in this report were collected on Form EIA-7A, "Cnal Production Report," from companies that produced, processed, or prepared coal in 1989. So that the EIA may fulfill its data collection functions as specified in the Federal Energy Administration Act of 1974 (Public Law 93-275), response to this survey is mandatory. The respondents to this survey are compared with lists of mining operations maintained by various State coal mining/licensing agencies and the Mine Safety and Health Administration (MSHA), U.S. Department of Labor, to identify new respondents. No sampling procedures are used. In 1989, there were 3,511 mining operations that produced, processed, or prepared 10,000 or more short tons of coal. Of these mining operations, 76.1 percent $(2,673)$ responded to the EIA-7A survey. In 1989, there were 2,821 mines that produced 10,000 or more short tons of coal. Of these mines, 73.1 percent $(2,063)$ responded to the EIA-7A survey and they accounted for $9(99.3$ million short tons, or 93 percent of the 1989 production total. All of the data were collected by mail and were edited to ensure that they were connplete and accurate.
The total number of U.S. mining operations and total U.S. coal production were taken from the 1989 EIA-7A data base, which included production by mines producing less than 10,000 short tons of coal during 1989. State mining agency reports on coal production for 1989 and Form 7000-2, "Quarterly Mine Employment and Coal Production Report," collectec by the Mine Safety and H:alth Administration, were used to determine production figures for EIA-7A nonrespondents.

As in all surveys, data from Form EIA-7A, "Coal Production Report," are subject to various sources of error: (1) coverage (the list of respondents may not be complete or, on the other hand, there may be double counting), (2) nonresponse (all units that are surveyed may not respond or may not provide all the information requested), (3) respondents (respondents may commit errors in reporting the data), (4) processing (the data collection agency may lose or incorrectly transcribe the submissions), (5) concept (the data collection elements may not measure the items they were intended to measure), and (6) adjustment (errors may be made in estimating values for missing data).

Because the annual coal production survey (Form EIA-7A) is not a sample survey, the estimates shown in this report are not subject to sampling error. ${ }^{1}$ It is not possible to present estimates of nonsampling error, but precautionary steps were taken at each stage of the survey design to minimize the possible occurrence of these errors. These steps are described below.

\section{Data Editing, Analysis and Processing}

The forms are logged within 24 hours of receipt and assigned to a team of editors consisting of Coal Data Systems Branch personnel and contractors. The editors screen the forms for legibility, completeness, and consistency. Names and address changes are updated in the files. The reported data are compared to data from previous years and with secondary sources such as

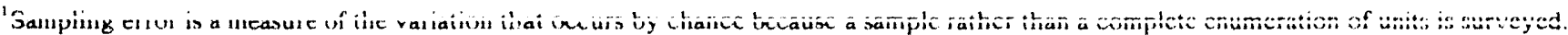


Mine Safety and Health Administration and State mining agency reports. Inconsistencies are identified and the respondent contacted for clarification.

Computer edits, re generated to identify keypunch errors, errors made by coders, out-of-range codes, and unlikely data com inations. Errurs are corrected to conform to the data in the submissions or revised after telephone conversations with company representatives. All changes to rep orted data are documented.

Data from Form EIA-7A ari maintained on an automated database at the EIA computing facility in Washington, D.C. The survey forms are filed by EIA identification number organized by State and county.

\section{Missing Data}

The survey forms were sent via regular mail in January with a due date of March 1, 1990. A nonresponse letter was mailed March 15 to those mining operations that had not submitted their forms. Subsequent to the letter, attempts were made to contact by telephone those nonrespondents whose reported 1988 production was 200,000 short tons or more.

Some forms could not be delivered. Where possible, address corrections were obtained. In some instances, mining operations that had reported in earlier surveys or operated only in 1989 were no longer in business. Unobtainable data were derived from three secondary sources: State mining agency coal production reports; the Form EIA-6 "Coal Distribution Report"; and Form 7000-2, "Quarterly Mine Employment and Coal Production Report," collected by the Mine Safety and Health Administration.

Missing production quantity data were derived from secondary sources for 1,187 mines, which produced 69.8 million short tons, or 7.1 percent of total production. The received and generated data sources for total U.S. coal production are shown in Table D1. Of the mines whose production exceeded 10,000 or nore short tons, missing production quantity data were derived from secondary sources for 758 mines, representing 7 percent ( 68.2 million short tons).

Missing coalbed classification, coalbed thickness, and coal rank/group data were estimated using State mining agency production reports, geological data, and previous years' reports for these mines.

When a mine had a missing production value, its production was multiplied by the county-level average mine price to estimate the value. County-level average mine prices were calculated by dividing the total value for the appropriate disposition (open/captive) and type of mining (underground or surface) by the corresponding total production. All missing production was classified as open market unless information was available to classify it as captive. Of those mines whose production was 10,000 or more short tons, data were estimated for 864 mines, representing 8.8 percent $(86.1$ million short tons) of their production total.

Employment data include direct labor hours, number of production days worked, average length of a production shift, average number of miners per shift, and average number of shifts per day. Of those mines whose production exceeded 10,000 or more short tons, one or more of these data elements were derived from secondary sources or estimated for 783 mines representing 7.2 percent (70.1 million short tons) of their production total. Of the mining operations that produced, processed or prepared 10,000 or more short tons, one or more of these data elements were derived from secondary sources or estimated for 877 mining operations, or 25 percent of the total.

Missing direct labor hours were estimated using countyor State-level productivity-per-hour averages. The averages were calculated by dividing the total production at the county or State level by total direct labor hours at the same level for the appropriate iype of mining (underground or surface). The quantity of production for the mine with missing data was divided by this productivity average to derive direct labor houss.

Missing average number of production days worked and length of a production shi,t were derived by using the corresponding county. or State-level average for the appropriate type of mining (underground or surface).

Missing average number of miners per shift was estimated as the average number of miners working daily. The average number of miners working daily was calculated by multiplying the average lerigth of a production shift by the number of production days worked, and then dividing this number into the total direct labor hours. Missing average number of shifts per day was estimated as one shift per day.

Missing recoverable coal reserves data were estimated by using the mine's 1988 recoverable reserves minus the mine's 1989 production. If this calculation could not be made, the mine's projected production for 1989 was used. If recoverable coal reserves for 1988 and 1989 and projected production for 1990 were all missing, no estimate was made. In 1989, recoverable reserves were reported by or estimated for 1,904 mines, representing 906.1 million short tons, or 92.7 percent whose production exceeded 10,000 or more short tons.

Missing recovery percentage data were estimated by using 1988 recovery percentage averages at the State level for the appropriate type of mining (underground or surface). 


\section{Table D1. Sources of Data for Total U.S. Coal Production' and Number of Mining Operations ${ }^{2}$}

(Thousand Short Tons)

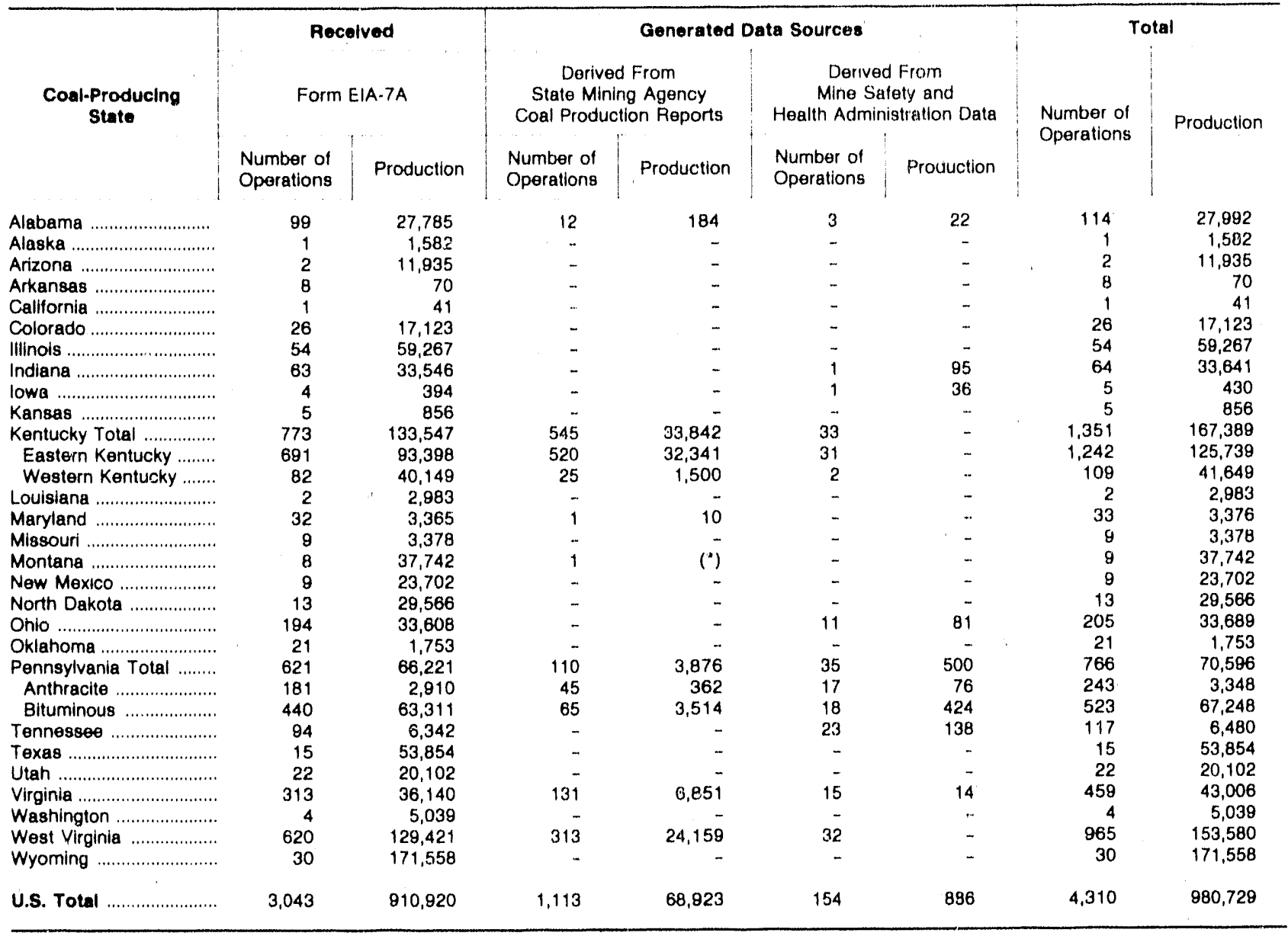

1 Excludes silt, culm, refuse bank, slurry dam, and dredge production except for Pennsylvania anthracite.

2 Includes preparation plants.

(") Less than 500 short tons.

Note: Totals may not equal sum of components because of independent rounding.

Sources: Energy Information Administration, Form ElA-7A, "Coal Production Report"; State Mining Agency Coal Production Reports; U.S. Department of Labor, Mine Safety and Health Administration, Form 7000-2, "Quarterly Mine Employment and Coal Production Report."

In 1989 , there wert 47 mines that produced 1.3 million short tons of refuse bituminous and subbituminous coal. These operations are not included in this report.

\section{Nondisclosure of Data}

In order to protect the confidentiality of individual respondent's da: a policy was implemented to ensure that the reportin f survey data on mine prices and recoverable reserves in this publication would not associate those data with a particular company. The final phase in the data quality as, Irance and control procedures is determining which data must be suppressed (withheld) during publication to provide the necessary confidentiality for mines or companies that operate in small reporting areas. All withholding analysis is done based upon production volumes. These procedures are performed as follows:

1. Primary Withholding Based on the Number of Respondents in a Cell -- All cells with three or fewer active coal mining operations are suppressed.

2. Residual Withholding Dominance Rule Phase 1 -- All cells containing between four and ten active coal mining operations are examined. A cell is suppressed if any single respondent accounts for 75 or more percent of the volume for all respondents included in that cell.

3. Residual Withholding Dominance Rule Phase 2 -- All cells in which two active coal mining operations represent 90 or more percent of the volume for all respondents included in that cell are suppressed. 
4. Complementary Suppression -- All tables are reviewed to identify cells which should have data withheld to prevent disclosure of already suppressed cells. An example of cells to be withheld during complementary suppression is underground price if the surface price is withheld during primary or residual suppression. Since the total price is published, if the underground price is not withheld, the surface price could be calculated using the total price, the underground price, and the underground, surface, and total volumes.

Most complementary suppression involves type of mining considerations. Other complementary suppression is based on regional level data. A State or Region must be withheld during complementary suppression because an already withheld state could be calculated using other States and the regional total. Cells are also selected for complementary suppression that represent the smallest volumes or that were withheld in prior years.

Inter-table effects are also examined regarding complementary suppression. For example, Districts and States that are withheld in one District or one State table can influence the complementary suppression of an associated State or District table. This occurs because some Districts equal some States or aggregates of States. This analysis is very similar to that done at the regional level except that two tables are involved rather than one. Finally, similar tables are reviewed to ensure that all like suppressed cells are consistently withheld (suppressed) in all tables in which they appear.

The withholding/suppression of data is performed as an adjunct to the Quality Assurance (QA) procedures. The work is performed by survey editors, and the QA staff and is reviewed by the survey manager before being submitted to division level QA review.

All sensitive cells identified in withholding analysis are denoted with the symbol/letter "W." The use of the symbol/letter applies to primary, complementary and inter-table suppressions as well as all withheld data. The symbol/letter " $W$ " is footnoted as follows: "W Withheld to avoid disclosure of individual company data."

\section{Data Revision and Correction Policy}

Published data are not revised. There is no new information that would become available, as the data have already been subjected to extensive quality assurance procedures and compared with secondary sources.

\section{Demonstrated Reserve Base}

\section{Sources of Data}

The demonstrated reserve base (DRB) has been derived over the past 20 years mostly from published sources such as U.S. and State geological survey and mining agency reports and reliable mineral assessments from various sources. The derivation of the coal resource data for each State DRB was documented in the EIA's 1979 DRB publication, along with comprehensive treatment of definitions and DRB background information. ${ }^{2}$ Subsequent revisions were documented in the EIA's 1980 DRB publication ${ }^{3}$ and in the DRB portions of Coal Production starting with the 1982 report.

Annual updates have involved one or more of three methods. The methods are: (1) depletion due to mining since the previous update; (2) revisions based on new data sources determined to be suitable under DRB criteria; and (3) adjustments using existing data sources as they relate to changes in DRB criteria or basic data analysis. The current update does not include any revisions due to new data sources or changes in DRB criteria or data analyses (methods 2 and 3 ). The various revisions the EIA has made to DRB data, from the 1979 through the 1989 updates, are listed in summary form in Table D2.

In recent years, new data on coal reserves have not been forthcoming in forms and aggregation levels appropriate for DRB revisions. In addition, the growing demands on DRB data for use in complex analyses have emphasized the need for better information on the characteristics and availability of DRB coal resources. Consequently, the EIA is currently initiating a new program to support the development and enhancement of coal resource data, with the cooperation of State and other coal resource authorities. This pro-

\footnotetext{
${ }^{2}$ Energy Information Administration, Demonstrated Reserve Base of Coal in the United States on January 1, 1979, DOE/EIA.0280(79) (Washington, DC, 1981).

${ }^{3}$ Energy Information Administration, Demonstrated Reserve Base of Coal in the United States on Janıary 1, 1980, DOE/EIA-1)280(80) (Washington, DC, 1982).
} 
gram is described more fully below (see "New Data Sources and Data Adjustments").

Appendix A presents data for the DRB on January 1, 1990. These data update the prior DRB estimate as of January 1, 1989.

\section{Depletion of Prior DRB Estimates}

Depletion adjustments are based on annual coal production data collected or estimate's by the EIA on Form EIA-7A for 1989. Each State DRB on January 1, 1990, was derived by adjusting the State DRB on January 1, 1989 (as shown in the publication Coal Production 1988), for depletion due to 1989 mining activity. No production from silt, culm, refuse bank, slurry dam, or dredge operations is included in annual depletion adjustments to the DRB because such sources represent portions of previous years' depletion. Reported production with no corresponding State level DRB (e.g. Texas bituminous coal production) is excluded from depletion adjustments.

\section{New Data Sources and Data Adjustments}

No new data sources are included in the 1989 DRB. All changes in data are the result of depletion adjustments based solely on recorded 1988 production. New resource data will be included in future updates.

In February 1990, the ElA held a Coal Reserves Assessment Conference in Arlington, Virginia, as a forum to discuss data needs and formulate a plan to improve national coal reserve estimates. The EIA is using information gained from the conference to plan a new program to develop updated, more detailed coal reserve estimates. The program would support States and alternative sources to process and compile available data that is needed for improved reserve base and recoverable reserve estimates. The program is being initiated with two pilot studies in Ohio and Wyoming, in cooperation with the Ohio Division of Geological Survey and tine Geological Survey of Wyoming. These studies were underway in October, 1990, to run for 1 year each.
Table D2. Summary of ElA Revisions to Demonstrated Reserve Base of Coal

\begin{tabular}{|c|c|c|}
\hline Year of Update & Action Taken 1 & $\begin{array}{l}\text { State or } \\
\text { Producing Area }\end{array}$ \\
\hline 1979 DRB & $\begin{array}{l}a, b, c \\
a, b, c \\
c \\
a, c \\
a, e \\
c, e \\
c, e, f \\
a, b \\
c, e \\
a, b, d \\
a, c \\
a, b, c, d, e \\
a, b, c, d, e \\
a, b \\
c \\
a, c, d, e\end{array}$ & $\begin{array}{l}\text { Alabama } \\
\text { Arizona } \\
\text { Arkansas } \\
\text { Georgia } \\
\text { W. Kentucky } \\
\text { Maryland } \\
\text { Michigan } \\
\text { Missouri } \\
\text { North Carolina } \\
\text { Oklahoma } \\
\text { South Dakota } \\
\text { Tennessee } \\
\text { Texas } \\
\text { Virginia } \\
\text { West Virginia } \\
\text { Wyoming }\end{array}$ \\
\hline 1980 DRB & $\begin{array}{l}e \\
a, c \\
a, e \\
c, f, g\end{array}$ & $\begin{array}{l}\text { Alabama } \\
\text { Colorado } \\
\text { Ohio } \\
\text { Washington }\end{array}$ \\
\hline $\begin{array}{l}1982 \text { DRB } \\
\text { (2-year update) }\end{array}$ & $\begin{array}{l}b \\
b, c \\
a, c \\
a, c \\
a, b, e\end{array}$ & $\begin{array}{l}\text { Alabama } \\
\text { Arkansas } \\
\text { Illinois } \\
\text { New Mexico } \\
\text { Texas }\end{array}$ \\
\hline 1983 DRB & $\begin{array}{l}a, f \\
a, c, d \\
c \\
a, b, e, f, g\end{array}$ & $\begin{array}{l}\text { Illinois } \\
\text { E. Kentucky } \\
\text { Pennsylvania (Bit.) } \\
\text { Texas }\end{array}$ \\
\hline 1984 DRB & $\mathrm{g}$ & $\begin{array}{l}\text { Pennsylvania (Anth.) } \\
\text { South Dakota }\end{array}$ \\
\hline 1985 DRB & $\begin{array}{l}f \\
d, g\end{array}$ & $\begin{array}{l}\text { E. Kentucky } \\
\text { Pennsylvania (Anth.) }\end{array}$ \\
\hline $\begin{array}{l}\text { 1986-87 DRB } \\
\text { (2-year update) }\end{array}$ & $a, e$ & Louisiana \\
\hline 1988 DRB & $\mathrm{h}$ & \\
\hline $\begin{array}{l}1989 \text { DRB } \\
1990 \text { DRB }\end{array}$ & $\begin{array}{l}\mathrm{h} \\
\mathrm{h}\end{array}$ & \\
\hline
\end{tabular}

1 Interpretations of "Action Taken" codes are as follows:

a. Incorporate new resource data

b. Reallocate resource data due to update of DRB criteria

c. Analysis of resource data

d. Adjust using revised depletion estimates

e. Analysis of depletion data

f. Corrections to resource data derivations

g. Corrections to depletion data adjustments

h. No new revisions, resource data adjustments, or corrections.

Note: Actions listed refer to revisions and adjustments due to new data or analyses, or corrections to existing data. Adjustments for depletion due to annual production are not listed. 


\section{Technical Notes}

\section{Resource Data and Minability Criteria}

The demonstrated reserve base, or DRB, of coal represents a level of coal resource classification (geologic assurance), with definitive physical measurements applied, that can supply a uniform and consistent data base for estimating minable in-place coal on a national basis. "Demonstrated" is a collective term for the sum of resource data in both the "measured" and "indicated" categories of geologic reliability (see the Glossary) and represents 100 percent of known measured and indicated coal in place as of a certain date. DRB coal occurs within depth and thickness ranges which are judged to be economically minable at the time of the estimate.

In applying unirorm standards nationwide, many sitespecific minability criteria must necessarily be passed over in order to maintain objectivity and compatibility of data. Some coalbeds that do not meet the "national" criteria for depth and thickness are included, however, because those or analogous beds are presently being mined.

The DRB is not a fix d quantity but one that will increase with discovery and new development, decrease with mining, and may change either upward or downward if the criteria for its calculation are modified. Additionally, both known and indeterminable factors such as natural and manmade obstructions, environmental and other legal restrictions, technology, and economics could limit or prevent (or encourage) the mining of certain coalteds. These factors not only affect the availability of DRB coal for mining, but also influence recoverability in the actual mining process.

\section{Coal Rank and Coalbed Characteristics}

The rank of coal is one of the more important physical criteria influencing its use and, hence, its commercial minability. The standard specifications for classifying coal by rank are presented in Appendix C.

In addition to rank of coal, quantitative data pertaining to thickness and depth of coalbeds are maintained for the DRB and identified resource data on file at the EIA. The DRB includes data for beds of bituminous coal and anthracite 28 or more inches thick and beds of subbituminous coal 60 or more inches thick that occur at depths having 1,000 feet or less of overlying material. Lignite beds 60 or more inches thick having 200 feet or less of overlying material are included. In addition, certain thinner and/or deeper resources are included that presently are being mined or for which there is evidence that they could be mined commercially at present. These criteria are defined in the 1979 publication and in the discussions in the "Explanatory Notes" section of the publication Coal Production, for each year from 1982 through the present.

\section{Depletion}

Depletion of DRB data is calculated by subtracting tonnages of coal mined and lost to mining since the previous update, or for another time period if prior depletion adjustments require re-evaluation. Depletion is computed from production statistics collected on Form EIA-7A and from certain generated data sources by applying depletion factors (multipliers), usually 2.00 and 1.25 , respectively, to underground and surfacemined production. Any coal that remains in the ground following termination of a mining operation has, in effect, been depleted from the DRB along with the recovered coal by use of these depletion factors. Some of the remaining coal, however, may be recovered later, and in instances where such "secondary" or even "tertiary" recovery is known, the depletion adjustments are revised to reflect the subsequent production.

Depletion factors are the reciprocal functions of recoverability estimates, expressed as percentages of the DRB or other quantified portions of a resource. The determination of recoverability percentages, referred to as "recovery factors," takes into consideration the fact that the quantity of coal recovered from the DRB varies from mine to mine because of a variety of factors that impede complete extraction, as discussed above. Also, an undetermined portion of coal, nationwide, is lost in cleaning processes following extraction, and is not included in production data.

For the purpose of calculating depletion rates only, standard recovery factors of 50 percent for underground mining and 80 percent for surface mining are selected by the EIA as reasonable estimates of "average" recovery rates for all ran ss of coal mined in the United States. This is based on past, present, and projected near-future 'nining practices, including regional recoverability studies by individuals with extensive coal industry experience, and on recoverability estimates submitted to the EIA from producers.

These standard rates will be used by the EIA until other rates become well established and documented. The only exceptional recovery rate being used for current production is a 90 percent factor for surface-mined Pennsylvania anthracite, where recovery reflects a combination of higher value coal and unusual geologic conditions.

The proportion of coal that can be recovered ("reserves") ranges from less than 40 percent in some underground mines to over 90 percent at some surface mines. The EIA believes that on a national basis at least one-half of the demonstrated in-place coal is recoverable at the present time. 


\section{Distinction Between Demonstrated Reserve Base Data and Recoverable Reserves as Reported on Form EIA-7A}

The demonstrated reserve base (as explained in Technical Note 1) provides a definitive and uniform basis for estimating minable in-place coal within the United States. At least one-half of this estimated tonnage is considered by the EIA to be recoverable nationwide at the present time. By contrast, "recoverable reserves," as reported on Form EIA-7A, are confined to producing mines and, as such, represent only a fraction of the DRB. (See the Glossary for relevant terms.)

There is no direct relationship between the DRB and the recoverable reserves reported on Form EIA-7A because the data come from different sources. The DRB, based on broad coal resource studies, is a compilation of regional coal resource estimates, with certain adjustments. The recoverable reserves from Form EIA-7A represent the summation of site-specific recoverable reserves within the boundaries or mining permit areas of active mines which report.

Conceptually, the relationship resembles a simple, broac'-patterned quilt (the DRB) with scattered de- tailed patchwork areas sewn on (the mine-site reserves). In some instances, the locations of mine-site reserves and DRB source studies may coincide only in part, or not at all. Further, the coalbeds covered in mine-site data and DRB source studies may be different. The DRB estimates represent coal still in the ground, whereas the recoverable reserves at active mines represent the tonnage that producers expect to extract from the mine sites. In terms of areas of coverage and typical detail of data, however, the quilt analogy is a useful description of how the two types of coal data relate to each other.

A key point regarding the two types of data is that the recoverable reserves at mines should not be expected to add up to the DRB. The recoverable reserves could be adjusted by the mines' various recovery factors and would describe coal reserves $i$, the ground, but only for a limited sampling - the mines active in the reporting year. Even if all the in-place reserves data from all past and future mines could theoretically be compiled, they would not equate to the DRB. In most cases they would, instead, supersede the DRB. The process of developing a mine in effect redefines (or in some cases defines for the first time) the actual DRB using ideally detailed data. The DRB published by the EIA is only an estimate. 


\title{
Appendix E
}

\author{
Form and \\ Instructions
}

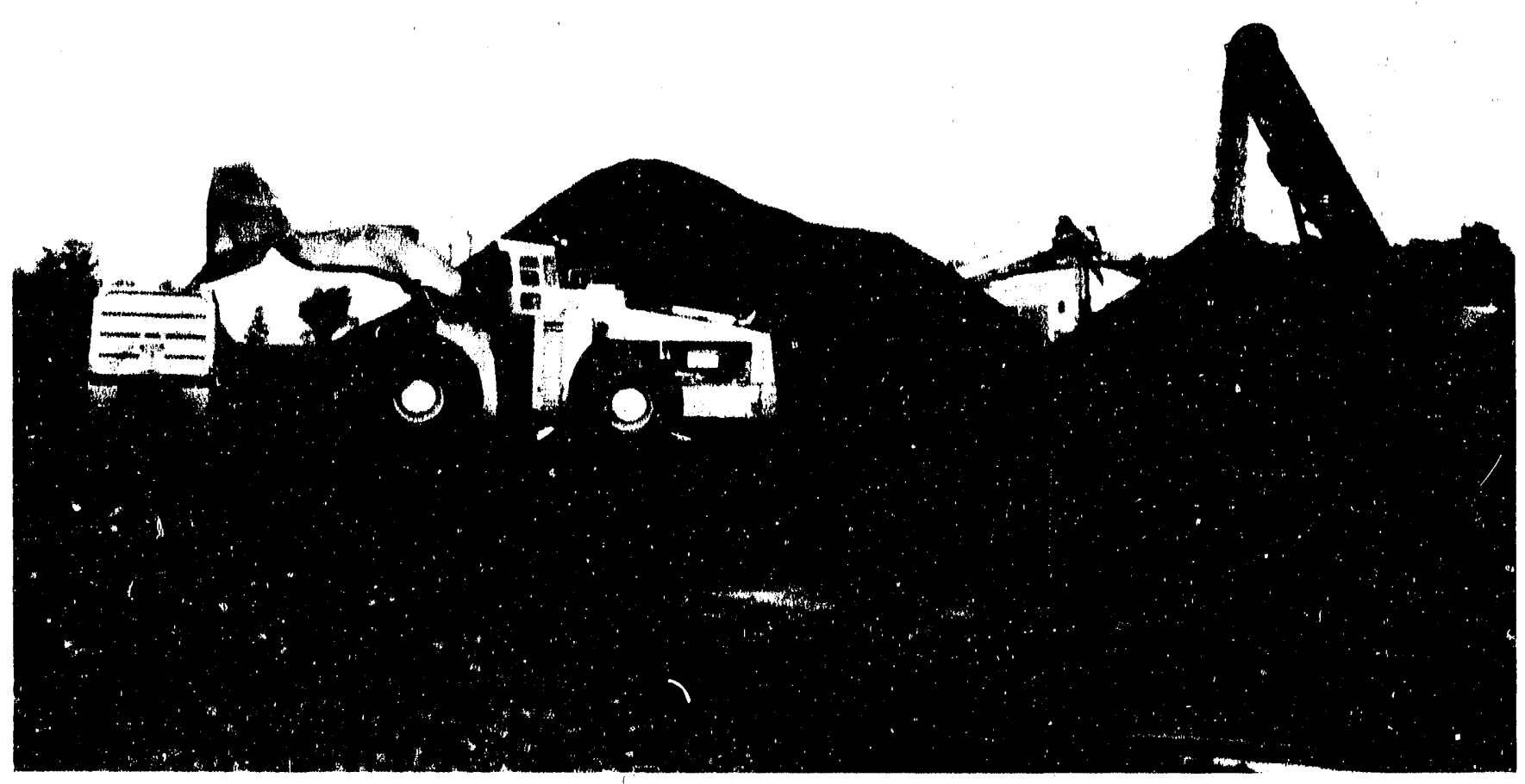

A belt-conveyor system stores clean coal at a West Kentucky preparation plant. 


\section{Appendix E}

U.S Department of Energy

Energy Information Administration

Form EIA-7A (OClober 1988)

\section{Coal Production Report}

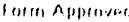

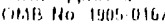

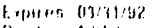
Burden 1 al hours

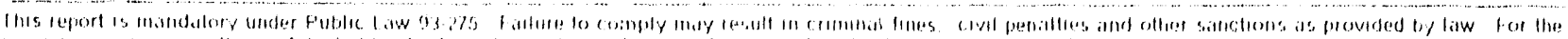

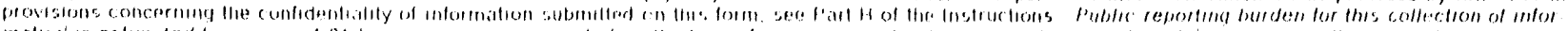

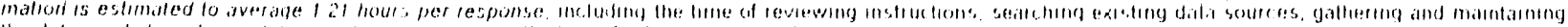

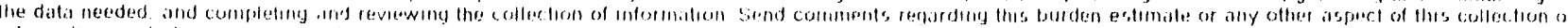

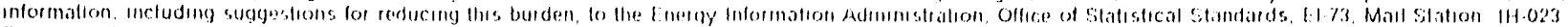

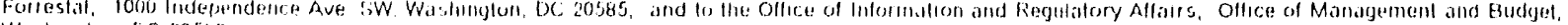
Washinglon, ic incoj

Please read the instructions provided before completing this form.

(Please make any corrections to the malling label in the area provided at the right and roturn the completed form in the bushliess reply envelope provided) GENERAL REPORTING INSTRUCTIONS: Form EIA-7A rnust be submitted by all coal minng companes that owned a minumg operation that produced, processed, or prepared coal during the reporting year. A separate form biA-7A must be submitted for each mining operation, l.e., mine and/or preparation plant, aridor tipple.

Mining operations that produced, processed, or prepared 10,00) or more short tons of coal should report as follows: (1) For mines only or mines with proparation plants or liphles, complete all parts of the form, (II) for preparation plarits and tupples only, complete Parts I, II. 3 and III through $V$ of the form.

If this mining operation produced, processed, or prepared under 10,000 short tons during the year, check this box, complete Sections I.(Identification), II.C.6 column (1) (Total Production Quantity), and V (Certification) and return this form in the enclosed envelope.

Operatirig subsidiaries and contractors who are unable to provide the data requested should forward Form ElA-7A to the parent cornipariy or contractee for completion prior to submission to the Energy Information Admunstration.

1. Identification the shaded areas in this section are reserved for preprinted iriformation. Please make any corrections to the information in these areas by drawing a line through the incorrect information and entering the changes in the space provided at the right.

A. Name and Location of Mining Operation

Mining Operation Name:

Location(County)

B. Name and Address of Operating Company

Location(State)

Company Name:

Acidress:

City, State, Zip:

C. Operating Company Point of Contact The operating company representative who can allswer questions reclarding the information provided on this form.

Name, Title:

Address:

City, State, Zip:

Phone No.(Area code):

D. Type of Company Operating this Mining Operation Check one. (See instructions for defiritions of company types.)
1. Independent Producer Operator
[.]2. Operating Subsidiary
[.] 3. Contractor

If you checked box 2 or 3 in section I.D above, fill in Sections I.E and I.F below. If there is more: than one Parent Company or Contractee, provide the additional intormation for these sections on a separale sheet of paper

E. Parent Company or Contractee Provide the following information about your Parent Company or Contractee.

Name of Parent/Contractee:

Address:

Cily, State, Zip:

F. Parent Company/Contractee Point of Contact Provide the following informalion atout your Paremt Company or Contractee contact person.

Name, Tille:

Addross:

Cily, Stale, Zip:

Phone No (Area Code) 


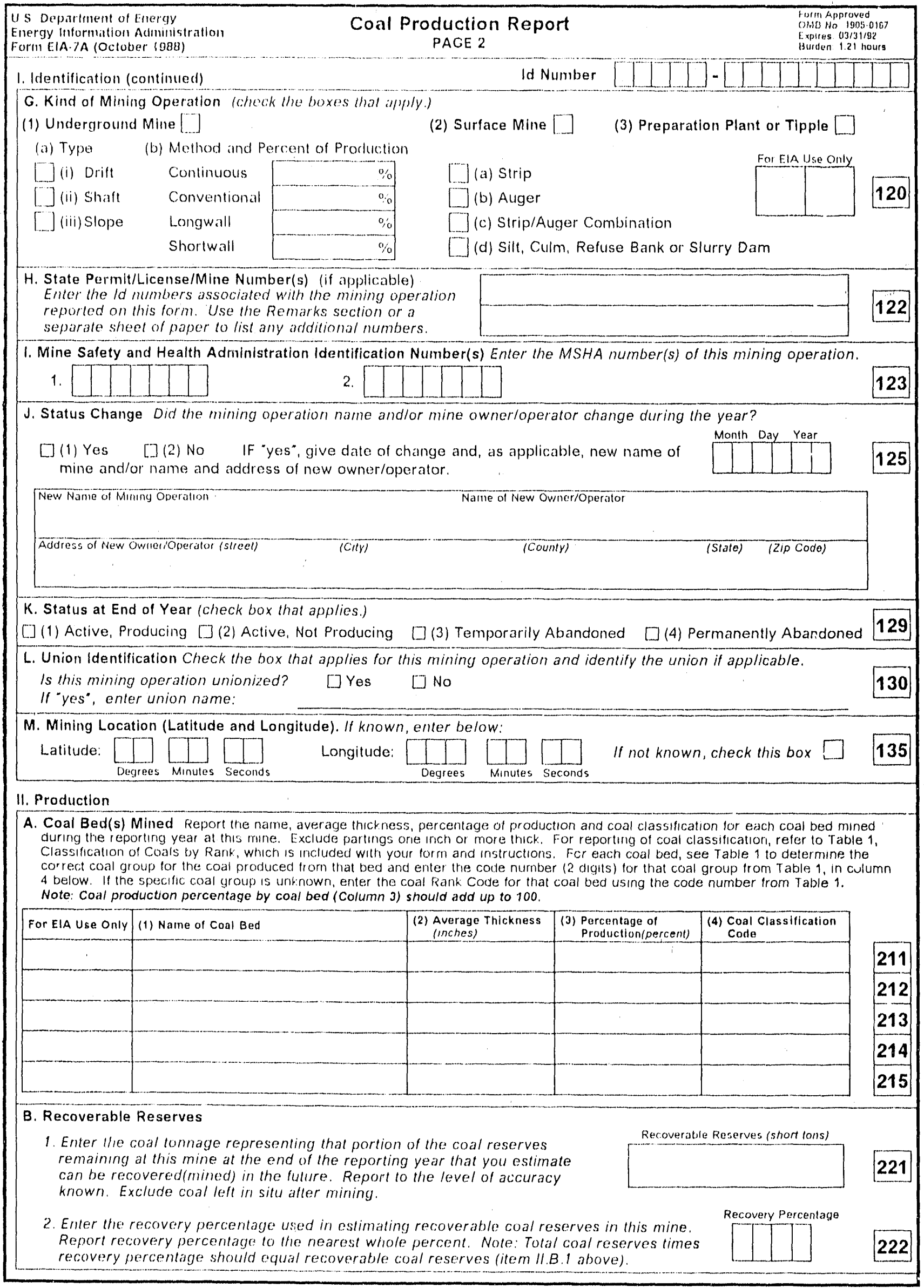




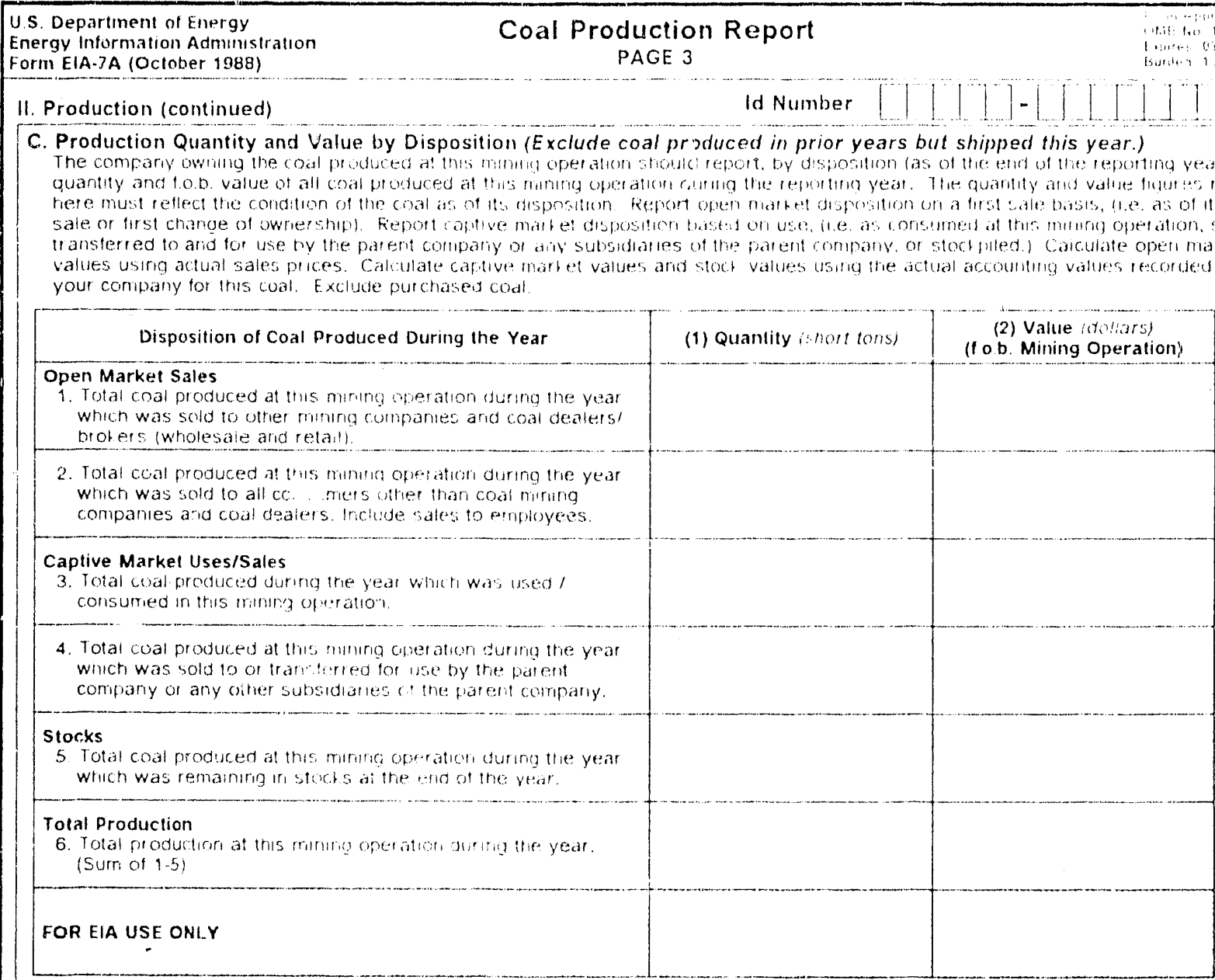

\section{Productivity}

A. Employment

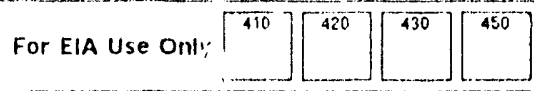

1. Total Direct Labor Hours Worked at this Mining Operation During the Vear (hours).

Report direct iabor hours for alt employees at tris minin? operation who were

engaged in productori, preparation, processirig, developmitn, mantenance.

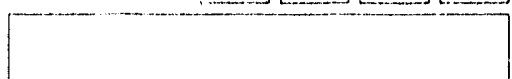

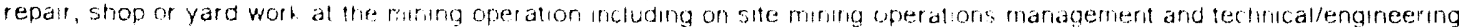
persorinel. Exclude office worters. For hourly employees, report hours actually wort ed and do not report vacation or leave hours. For salaried employees, repon actial or best estimate of hifurs wort ed Exclude reclamation hours.

2. Total Number of Production Days Worked at thi : Mining Operation During the Year (days). A production day is ariy day in whith coal was mined, and/ct loaded tor stmpment, andior stoct pled.

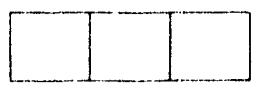

3. Average Number of Employees per Shift Compute ty dding the total number of employees worting each shitt. whether it is a production or a mainterabice shitt, and dividing this total by the total iumber of shitte worted per day. Average over the total number of

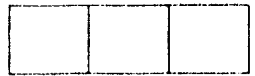
days worted. Fourd is the nearest whole hurricer.

4. Average Number of Shifts per Day. Averaue civer the lotal number of day. worted. Include manteriance as well as production shits. Repolt in tre nearest lentti of a shift lle. $1.0,2.2,3.5$,

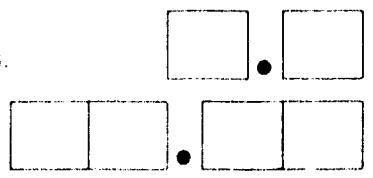

5. Average Length of Production Shift itows Repor: ro the rearest hurdredth of an tiour. (For example, $7^{\%}=0725,0^{\%}=08.501$. Iricilde ovatime if usually worted auring tre year

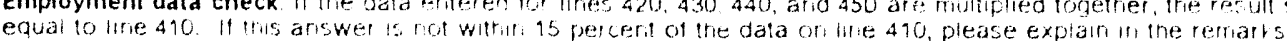

B. Daily Productive Capacity and Projected Production

1. Daily productive capacity of this mining operation (sholt tons/day)

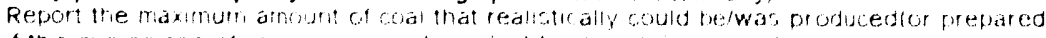

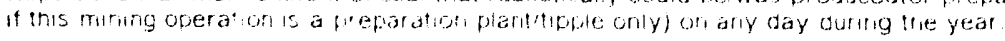

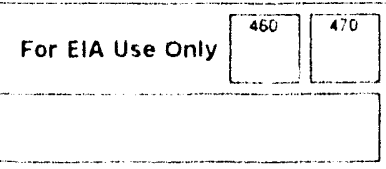

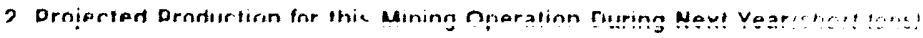




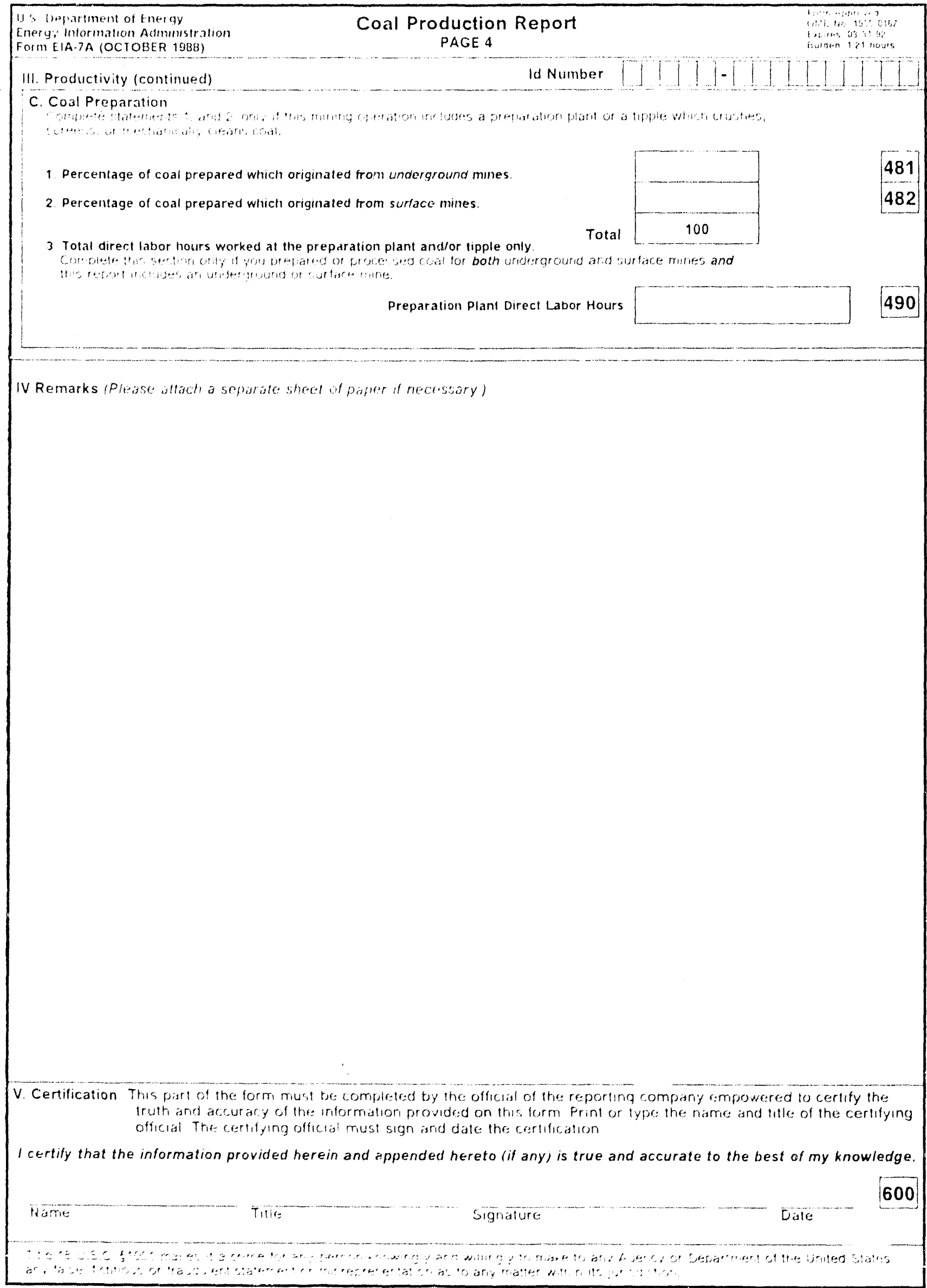


In:tructions for completing Form EIA.7A. "Coal Production Report"

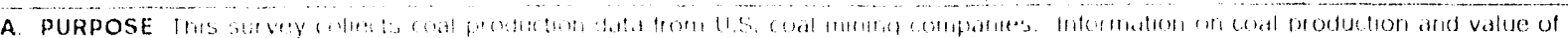

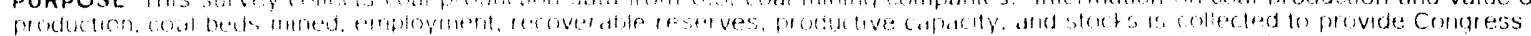

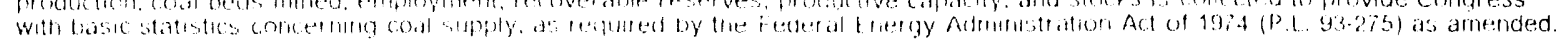

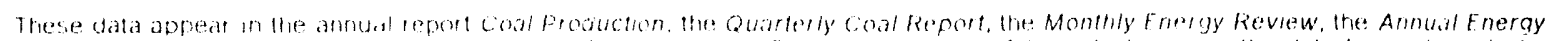

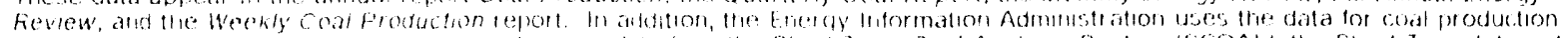

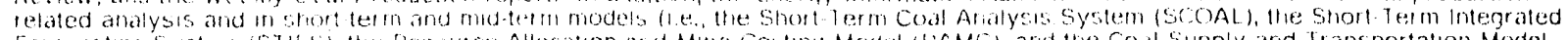

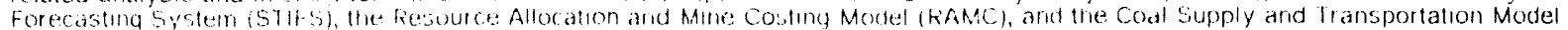

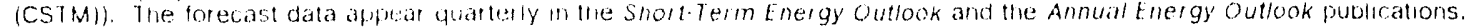

B. WHO MUST SUBMIT FoIm EIA.7A must be submitted by all cout mining companes that owned a mung operation that produced, pro-

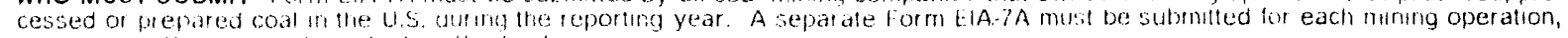
1.e., mine and/or preparalion plant and/or lipple.

C. WHERE TO SUBMIT Form EIA.7A should be returried in the business reply envelope provided to:

Energy Information Admuristration, El-521

Man Slatum: BG-044,

U.S. Departirent of treegy

Was hington, D.C. 20585

Alt?: EIA-TA

Fequesis for fut the information, additional forms, or business reply envelopes should be directed to the address above or by telephone on (202) 2545400 .

D. WHEN TO SUBMIT Form ElA-7A must be submitted by March 1 to report data tor the previous year.

E. SANCTIONS The timely subimssion of Form EIA-7A by a company equired to report is a mandatory requirement. Late filıng, fallure to file, or faliure otherwise to make information avalable to ElA in accordance with these instructions may result in crimunal fines, civil penalties and other sanctions as provided by Section 1311) of the FEA Act (P.L. 93.275).

\section{F DEFINITIONS}

Auger Mine: A surtace mine in which the coat bed is removed by means of a large diameter drill. Usually operated only when the overburden becomes too inick for economical strip mining.

Captive Coal: All coal produced and consumed by the independent producer/operator or opet atıng subsidiary or produced for consumption by a parent company.

Coal Bed: A bed or stratum of coal. Also called a coal seam.

Drift Mine: A mine that operis horizuntally into the coal bed or coal outcrop.

f.o.b. (Free on Board) Mining Operation Value: The price of coal at the mining operation. Does not include insurance and freight or shippirig cosis.

Independent Producer/Operator: A compariy which operates a coal mining operation and has no subsidiaries or parent companies and is not a contractor

Latitude and Longitude: The distance on the earth's surface measured, respectively, north or south of the equator and east or west of the slandard meridian, expressed in angular degrees. minutes, and seconds.

Mining Operation: Ore mine and/or tipple at a smgle physical tocation.

MSHA ID Number: Seven (7) digit code assigned to a mining operation by the Mine Safety and Health Administratıon.

Open Market Coal: Coal sold in the open martet (t.e., coal sold to companies other than the reporting company's parent company or an operating subsidiary of the parent company).

Operating Subsidiary: Company which operates a coal minıng operation and is owned by another company (I.e., the parent company).

parent Company: A company which solely or fointly owns a subsialary which operates a coal minng operation and is not owned itsell by another company.

Report Year: The calendar year begirinng at 12:00 a.m. January 1 and ending at 11:59 p.m. December 31

Shaft Mine: A mirie that reachies the coal bed by means of a vertical shaft.

Slope Mine: A mine that reaclies the coal bed by means of an inclined opening.

State Permit/License/Mine Number: Code assignted to a minng operation by thie state in which the operation is located.

Sirip Mine. An coperi cut in whichi the overburden is removed from a coal bed prior to the removal of coal.

G. GENERAL INSTRUCTIONS Miring operations that produced, processed or prepared less than 10,000 short tons of coal during the year should chect the first box (Line Code 110) on the form and complete Sections I (Identıficatıon), II.C.6 column (1) (Total Production Quantity), arid V (Certification). Mines only or miries with preparation plants or tipples that prodisced 10,000 or more short tons during the year should corriplete all parts of the form. Freparation plants or tipples only that prepared or processed 10,000 or more stiont tons of coal during the year should complete Parts 1, 11.C.3 arid III thirough $V$ of the form. Repoit coal quaritties in short tons 12,000 pounds) to the nearest whole ton. Repont value in dollars to the nearest dollar. Report coal bed thickness in inches, to the riearest whote inch. Report perceritages to the nearest whole percent. Operating subsidiaries arid contractors who are unable to provide the data requested should forward form ElA-7A immediately to the parent company or contractee for completion prior to sutumission to the Eriergy Information Admunistration.

H. DISCLOSURE OF INFORMATION The following data elements will not be treated as confidenflal by ElA:

a. Total production quantıty

b. Coat beds mined.

The name and address of the respondirig company, the mue or plant type (1.e., underground, surface, preparation plant) and tocation (state, courity, andor coal-producing districl) will be leteased upon request in the form of a fult or partial list of respondents for this for tris coal data collection survey. All other iriformation, other than that des cribed above, will be tept confidential to the extent that it satisties the criteria for exemintom in the Freedorm of intormation Act ifOAA), the DOE regulations implemenitirig the FOIA, and the Trade Secrets ACt, 18 U.S.C. $\$ 1905$.

Upon receipt of a request for this irifm mation under the FOIA, the DOE shall, in accordance with the procedures and crteria provided in 10 C.F.R. \$1004.11, make a tinal determiniation whether the iniformation is exempt from disciosure. To assist in this determination, respon dents should demonstrate to the DOE that, for example, their information contains trade secrets or commercial or financial information whose release would be lit ely 10 cause substatial harm to then compariys competilive position. A letter accompanying the submission that explans (on an element-by-elethent bask) the teasons why the information would be litely to cause the respondent substantial competitive harm if released to the puthlic would ald in this determination. A nevi justilication does riot need to be provided each lirne information is submitted on the form if the company has previousily subritted a justification for that information and the justification has not beer chariged.

Excen as otrerwise provided by liw. the information may be riade avalable in response to an order of a Coun of competent jurisdic.

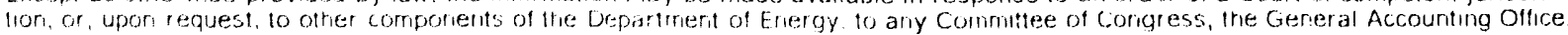
or other congressional auehicies duthionted by law to tecelve such inturmation. Detalted provisions of the restrictions on the disclosure

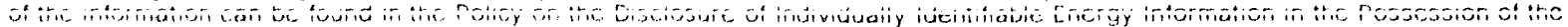

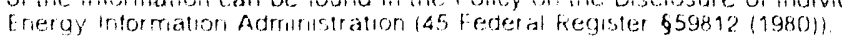




\section{Glossary}

Agglomerating Character: Agglomeration describes the caking properties of coal. Agglomerating character is determined by examination and testing of the residse when a small powdered sample is heated to 950 degrees centigrade under specified conditions. If the sample is "agglomerating," the residue will be coherent, show swelling or cell structure, and be capable of supporting a 500-gram weight without pulverizing.

Anthracite: Anthracite, or hard coal, is the highest rank of economically usable coal. It is jet black with a high luster. The moisture content generally is less than 15 percent. Anthracite contains approximately 22 to 28 million Btu per ton as received and averages about 25 million Btu per ton. Its ignition temperature is approximately 925 to 970 degrees Fahrenheit. Virtually all of the anthracite mined in the United States is from northeastern Pennsylvania. It is used mostly for space heating and generating electricity.

Auger Mine: A mine in which coal is recovered through the use of a large-diameter drill driven into a coalbed in a hillside. It usually follows contour surface mining, particularly when the overburden is too costly to excavate.

Average Daily Production: The ratio of the total production at a mining operation to the total number of production days worked at the operation.

Average Length of a Shift: The arithmetic mean number of hours worked during a production shift. Overtime is included if usually worked during the year.

Average Mine Price: The ratio of the total value of the coal produced at the mine to the total production tonnage. (See f.o.b. mine price.)

Average Number of Employees per Shift: The arithmetic mean number of employees working during a production shift. Includes all employees except office workers. (See direct labor hours.)

Average Number of Shifts per Day: The arithmetic mean number of shifts each day at a mining operation. Includes maintenance as well as production shifts.

Average Production per Miner per Day: The product of the average production per miner per hour at a min- ing operation and the average length of a production shift at the operation.

Average Production per Miner per Hour: The ratio of the total production at a mining operation to the total direct labor hours worked at the operation.

Bituminous Coal: Bituminous coal, or soft coal, is the most common coal. It is dense, black, often with welldefined bands of bright and dull material. Its moisture content usually is less than 20 percent. The heating value ranges from 19 to 30 million Btu per ton as received and averages about 24 million Btu per ton. The ignition temperature ranges from about 700 to almost 900 degrees Fahrenheit. Bituminous coal is mined chiefly in the Appalachian and Interior coal fields. It is used for generating electricity, making coke, and space heating.

Btu (British thermal unit): The amount of heat needed to raise the temperature of 1 pound of water by 1 degree Fahrenheit. The Btu is a convenient measure by which to compare the energy content of various fuels.

Captive Coal: Coal produced and consumed by the mine operator, a subsidiary, or parent company (for example, steel companies and electric utilities).

Coal Preparation: The process of sizing and cleaning coal to meet market specifications by removing impurities such as rock, sulfur, etc. May include crushing, screening, or mechanical cleaning.

Coal-Producing Districts: A classification of coalfields defined in the Bituminous Coal Act of 1937. The districts were originally established to aid in formulating minimum prices of bituminous and subbituminous coal and lignite. Because much statistical information was compiled in terms of these districts, their use for statistical purposes has continued since the abandonment of that legislation in 1943. District 24 was added for the anthracite-producing district in Pennsylvania.

Coal-Producing Regions: A geographic classification of coal-producing States. The States in the Appalachian Region are Alabama, Georgia, Eastern Kentucky, Maryland, Ohio, Pennsylvania, Tennessee, Virginia, and West Virginia. The States in the Interior Region are Arkansas, Illinois, Indiana, Iowa, Kansas, Western Kentucky, Louisiana, Misouri, Oklahoma, and Texas. The States in the Western Region are Alaska, Arizona, 
California, Colorado, Montana, New Mexico, North Dakota, Utah, Washington, and Wyoming.

The three regional groupings by district are as follows:

Appalachian: Coal-Producing Districts 1-4, 6-8, 13, 24

Interior: Coal-Producing Districts 5, 9-12, 14-15

Western: Coal-Producing Districts 16-23.

Coal Rank: A classification of coal based on fixed carbon, volatile matter, heating value, and agglomerating character. It is an indication of the progressive alteration, or coalification, from lignite to anthracite. The rank of coal can also be determined by measuring the reflectance of vitrinite, one of the several organic components (macerals) of coal.

Coal Stocks: The supply of coal remaining at the mine, preparation plant, or tipple at the end of the reporting period.

Coalbed: A bed or stratum of coal. Also called a coal seam.

Continuous Mining: A form of room-and-pillar mining in which a continuous mining machine extracts and removes coal from the working face in one operation; no blasting is required.

Conventional Mining: The oldest form of roomand-pillar mining which consists of a series of operations that involve cutting the coalbed so it breaks easily when blasted with explosives or high-pressure air, and then loading the broken coal.

Culm: A mixture of very fine coal and refuse. Synonymous with silt.

Demonstrated Reserve Base: A collective term for the sum of coal in both measured and indicated resource categories of reliability which represents 100 percent of the coal in these categories in-place as of a certain date. Includes beds of bituminous coal and anthracite 28 or more inches thick and beds of subbituminous coal 60 or more inches thick that occur at depths to 1,000 feet. Includes beds of lignite 60 or more inches thick that can be surface mined. Includes also thinner and/or deeper beds that presently are being mined or for which there is evidence that they could be mined commercially at this time. Represents that portion of the identified coal resource from which reserves are calculated.

Depletion: The subtraction of both the tonnage produced and the tonnage lost to mining from identified resources to determine the remaining tonnage as of a certain time.

Depletion Factor: The multiplier applied to the tonnage produced to compute depleton. This multplier takes into account both the tonnage recovered and the ton nage lost due to mining. The depletion factor is the reciprocial of the recovery factor in relation to a given quantity of production.

Direct Labor Hours: Direct labor hours worked by all mining employees at a mining operation during the year. Includes hours worked by those employees engaged in production, preparation, development, maintenance, repair, shop or yard work, management, and technical or engineering work. Excludes office workers. Excludes vacation and leave hours.

Dredge Mining: A method of recovering fine coal from rivers or streams.

Drift Mine: An underground mine that has a horizontal entry dug to a coalbed in a hillside.

Dry (Coal) Basis: Coal quality data calculated to a theoretical basis in which no moisture is associated with the sample. This basis is determined by measuring the weight loss of a sample when its inherent moisture is driven off under controlled conditions of low temperature air-drying followed by heating to just above the boiling point of water (104 to 110 degrees centigrade).

Fixed Carbon: The nonvolatile matter in coal minus the ash. Fixed carbon is the solid residue other than ash obtained by prescribed methods of destructive distillation of a coal. Fixed carbon is the part of the total carbon that remains when coal is heated in a closed vessel until all volatile matter is driven off.

f.o.b. Mine Price: The free on board mine price. This is the price paid for coal measured in dollars per short ton at the mining operation site and therefore does not include freight or shipping and insurance costs.

Identified Resources: Coal deposits whose location, rank, quality, and quantity are known from geologic evidence supported by engineering measurements. Included are beds of bituminous coal and anthracite 14 or more inches thick and beds of subbituminous coal and lignite 30 or more inches thick that occur at depths to 6,000 feet. The existence and quantity of these beds have been delineated within specified degrees of geologic assurance as measured, indicated, or infurred. Also included are thinner and/or deeper beds that presently are being mined or for which there is evidence that they could be mined commercially.

Indicated Resources: Coal for which estimates of the rank, quality, and quantity have been computed partly from sample analyses and measurements and partly from reasonable geologic projections. Indicated resources are computed partly from specified measurements and partly from projection of visible data for a reasonable distance on the basis of geologic evidence. The points of observation are $1 / 2$ to $1-1 / 2$ miles apart. Indicated coal is projected to extend as a $1 / 2$-mile wide be!t that lies more than 1/4 mile from the outeron or points of observation or measurement. 
Inferred Resources: Coal in unexplored extensions of demonstrated resources for which estimates of the quality and size are based on geologic evidence and projection. Quar'itative estimates are based largely on broad knowledge of the geologic character of the bed or region and where few measurements of bed thickness are available. The estimates are based primarily on an assumed continuation from demonstrated coal for which there is geologic evidence. The points of observation are $1-1 / 2$ to 6 miles apart. Inferred coal is projected to extend as a $2-1 / 4$-mile wide belt that lies more than $3 / 4$ mile from the outcrop or points of observation or measurement.

Lignite: Lignite, the lowest rank of coal, is brownish black and has a high moisture content, sometimes as high as 45 percent. It tends to disintegrate when exposed to the weather. The heat content of lignite ranges from 9 to 17 million Btu per ton as received and averages about 14 million Btu per ton. The ignition temperature is approximately 600 degrees Fahrenheit. Lignite is mined in California, Louisiana, Montana, North Dakota, and Texas, and is used mainly to generate electricity in power plants that are relatively close to the mines.

Longwall Mining: A form of underground coal mining which is gaining in importance in the United States and can be used at greater depths than room-and-pillar mining. In longwall mining, a cutting machine is pulled back and forth across a panel of coal 300 to 6,00 feet wide and as much as a mile long, with the broken coal moved by conveyor. Longwall mining is done under movable rouf supports that are advanced as the bed is cut. The roof in the mined-out area is allowed to fall as the mining advances.

Measured Resources: Coal for which estimates of the rank, quality, and quantity have been computed, within a margin of error of less than 20 percent, from sample analyses and measurements from closely spaced and geologically well-known sample sites. Measured resources are computed from dimensions revealed in outcrops, trenches, mine workings, and drill holes. The points of observation and measurement are so closely spaced and the thickness and extert of coals are so well defined that the tonnage is judged to be accurate within 20 percent. Although the spacing of the points of observation necessary to demonstrate continuity of the coal differs from region to region according to the character of the coalbeds, the points of observation are no greater than 1/2 mile apart. Measured coal is projected to exiend as a 1/4-mile wide belt from the outcrop or points of observation or measurement.

Mine Count: The number of mines, or mines collocated with preparation plants or tipples, located in a particular geographic area (State, region, or coal-producing district). If a mine is mining coal across two counties within a State or across two States, then it is counted as two operations. This is done so that EIA can separate production by State and county.
Mineral-Matter-Free Basis: The parent material in coal from which ash is derived, and which comes from minerals present in the original plant materials that formed the coal, or from extraneous sources such as sediments and precipitates from mineralized water is called the mineral matter. Mineral matter in coal cannot be analytically determined and is commonly calculated using data on ash and ash-forming constituents. Coal analyses are calculated to the mineral-matter-free basis by adjusting formulas used in calculations in order to deduct the weight of mineral matter from the total coal.

Moist (Coal) Basis: "Moist" coal contains its natural inherent or bed moisture, but does not include water adhering to the surface. Coal analyses expressed on a moist basis are performed or adjusted so as to describe the data wh". the coal contains only that moisture which exists in the bed in its natural state of deposition, and when the coal has not lost any moisture due to drying.

Open Market Coal: Coal production that is sold on the open market to other coal companies or directly to consumers.

Overburden: Any material, consolidated or unconsolidated, that overlies a coal deposit.

Percent Utilization: The ratio of the average daily production to the daily productive capacity.

Preparation Plant: A mining facility at which coal is crushed, screened, and mechanically cleaned.

Recoverable Reserves: The amount of coal that can be recovered (mined) from the coal deposits at active, producing mines as of the end of the year, as reported on Form EIA-7A.

Recovery Factor: The percentage of total tons of coal estimated to be recoverable from a given area in relation to the total tonnage estimated to be in the demonstrated reserve base. The estimated recovery factors for the demonstrated reserve base generally are 50 percent for underground mining methods and 80 percent for surface mining methods. More precise recovery factors can be computed by determining the total coal in place and the total recoverable in any specific locale.

Recovery Percentage: The percentage of coal that can be recovered from the coal deposits at existing mines.

Refuse Bank: A repository for waste material generated by the coal cleaning process.

Refuse Mine: A surface mine where coal is recovered from previously mined coal. It may also be known as a silt bank, culm bank, refuse bank, slurry dam, or dredge operation.

Reserve: That portion of the demonstrated reserve base that is estimated to be recoverable at the time of de- 
termination. The reserve is derived by applying a recovery factor to that component of the identified coal resource designated as the demonstrated reserve base.

Room-and-Pillar Mining: The most common method of underground mining in which the mine roof is supported mainly by coal pillars left at regular intervals. Rooms are places where the coal is mined; pillars are areas of coal left between the rooms. Room-and-pillar mining is done either by conventional or continuous mining.

Shaft Mine: An underground mine equipped with elevators that provide vertical access to a coalbed that is generally deeper than one reached by a slope mine. In addition to the passages providing entry to the coalbed, a network of other passages are also dug, some to provide access to various parts of the rnine and some for ventilation.

Shortwall Mining; A form of underground mining that involves the use of a continuous mining machine and movable roof supports to shear coal panels 150 to 200 feet wide and more than half a mile long. Although similar to longwall mining, shortwall mining is generally more flexible because of the smaller working area. Productivity is lower than with longwall mining because the coal is hauled to the mine face by shuttle cars as opposed to conveyors.

Silt: A mixture of very fine coal and refuse. Synonymous with culm.

Slope Mine: An underground mine in which the entry is driven at an angle to reach the coal deposit.

Slurry Dam: A repository for the silt or culm from a preparation plant.
Strip (Surface) Mining: A method used on flat terrain to recover coal by mining long strips successively. The material excavated from the strip being mined is deposited in the strip previously mined.

Subbituminous Coal: Subbituminous coal, or black lignite, is dull black and generally contains 20 to 30 iercent moisture. The heat content of subbituminous coal ranges from 16 to 24 million Btu per ton as received and averages about 18 million Btu per ton. Subbituminous coal, mined in the western coal fields, is used for generating electricity and space heating.

Surface Mine: A coal-producing mine that is usually within a few hundred feet of the surface. Earth and rock above or around the coal (overburden) is removed to expose the coalbed, which is then mined with surface excavation equipment such as draglines, power shovels, bulldozers, loaders, and augers. It may also be known as an area, contour, open-pit, strip, or auger mine.

Tipple: A loading facility at the mine where coal is crushed or screened prior to cleaning.

Underground Mine: A mine where coal is produced by tunneling into the earth to the coalbed, which is then mined with underground mining equipment such as cutting machines and continuous, longwall, and shortwall mining machines. Underground mines are classified according to the type of opening used to reach the coal, i.e., drift (level tunnel), slope (inclined tunnel), or shaft (vertical tunnel).

Volatile Matter: Those products, exclusive of moisture, given off by a material as gas or vapor. Volatile matter is determined by heating the coal to 950 degrees centigrade under carefully controlled conditions and measuring the weight loss, excluding weight of moisture driven off at 105 degrees centigrade. 

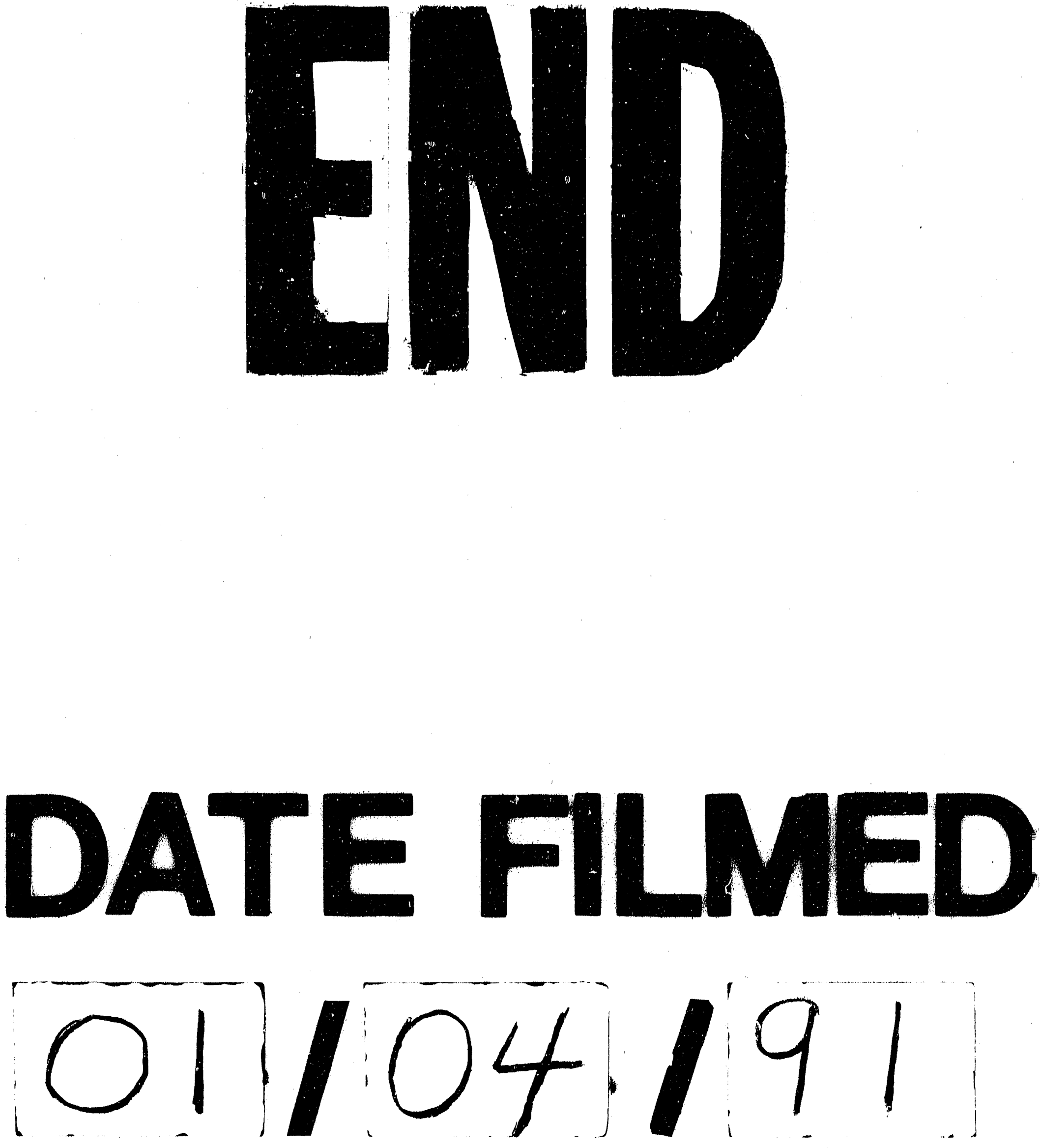
